\title{
Brain in sight : probing the neural dynamics underlying conscious vision
}

Citation for published version (APA):

de Graaf, T. A. (2013). Brain in sight : probing the neural dynamics underlying conscious vision. [Doctoral Thesis, Maastricht University]. Maastricht University. https://doi.org/10.26481/dis.20130315tg

Document status and date:

Published: 01/01/2013

DOI:

10.26481/dis.20130315tg

Document Version:

Publisher's PDF, also known as Version of record

\section{Please check the document version of this publication:}

- A submitted manuscript is the version of the article upon submission and before peer-review. There can be important differences between the submitted version and the official published version of record.

People interested in the research are advised to contact the author for the final version of the publication, or visit the DOI to the publisher's website.

- The final author version and the galley proof are versions of the publication after peer review.

- The final published version features the final layout of the paper including the volume, issue and page numbers.

Link to publication

\footnotetext{
General rights rights.

- You may freely distribute the URL identifying the publication in the public portal. please follow below link for the End User Agreement:

www.umlib.nl/taverne-license

Take down policy

If you believe that this document breaches copyright please contact us at:

repository@maastrichtuniversity.nl

providing details and we will investigate your claim.
}

Copyright and moral rights for the publications made accessible in the public portal are retained by the authors and/or other copyright owners and it is a condition of accessing publications that users recognise and abide by the legal requirements associated with these

- Users may download and print one copy of any publication from the public portal for the purpose of private study or research.

- You may not further distribute the material or use it for any profit-making activity or commercial gain

If the publication is distributed under the terms of Article $25 \mathrm{fa}$ of the Dutch Copyright Act, indicated by the "Taverne" license above, 


\section{Brain in Sight:}

probing the neural dynamics underlying conscious vision

Tom Alexander de Graaf 
Cover Design \& layout: T.A. de Graaf Production: Wöhrman Print Service

(C) T.A. de Graaf, Maastricht

The work presented in this thesis was supported by the Toptalent program (\#021-002-087) of Netherlands Organization for Scientific Research (NWO) and was largely conducted at Faculty of Psychology and Neuroscience, Maastricht University, Maastricht, the Netherlands 


\title{
Brain in Sight:
}

\section{probing the neural dynamics underlying conscious vision}

\author{
Dissertation \\ To obtain the degree of Doctor at the Maastricht University, \\ on the authority of Rector Magnificus, Prof. dr. L.L.G. Soete, \\ in accordance with the decision of the Board of Deans, \\ to be defended in public on Friday $15^{\text {th }}$ of March 2013 at 10.00 hours
}

by

Tom Alexander de Graaf 


\section{Supervisors}

Prof. dr. A.T. Sack

Prof. dr. R. Goebel

\section{Assessment Committee}

Prof. dr. B. Jansma (Chair)

Prof. dr. E. Formisano

Prof. dr. J. Silvanto (Aalto University, Finland)

Prof. dr. P. de Weerd

Dr. K. Uludag 


\section{Table of Contents}

Prologue

P1 The "Correlates" in Neural Correlates of Consciousness

Part I: Low-level Aspects of Vision: the disrupted rivalry effect

I.1 The Disrupted Rivalry Effect

I.2 Characterizing the Disrupted Rivalry Effect (I): the prevalence and duration of disappearing stimuli under various conditions

I.3 Characterizing the Disrupted Rivalry Effect (II): decreased DRE duration by gradual stimulus onset and offset

I.4 Characterizing the Disrupted Rivalry Effect (III): "true" disrupted rivalry

Part II: Low-level Aspects of Vision: TMS Masking

II.1 A Chronometric Exploration of High-resolution 'Sensitive TMS Masking' Effects on Subjective and Objective Measures of Vision

II.2 Feedforward and Quick Recurrent Processes in Early Visual Cortex Revealed by TMS?

II.3 On the Masking Effects of Occipital TMS with Varying Intensity when Applied Before and After Visual Stimuli (I)

II.4 On the Masking Effects of Occipital TMS with Varying Intensity when Applied Before and After Visual Stimuli (II)

II.5 The Chronometry of Visual Perception: review of occipital TMS masking studies

\section{Part III: High-level Aspects of Vision}

III.1 On the Functional Relevance of Frontal Cortex for Passive and Voluntarily Controlled Bistable Vision

III.2 Null Results in TMS: from absence of evidence to evidence of absence

III.3 Alpha-band Rhythms in Visual Task Performance: phase-locking by rhythmic sensory stimulation

III.4 Brain Network Dynamics Underlying Visuospatial Judgment: An fMRI Connectivity Study

Summary

Samenvatting

Publications 



\section{General Introduction}

All your thoughts, all your feelings, all your dreams and hopes, are built, caused, represented and stored, in a 3-pound pudding you could hold in one hand if only it weren't so securely locked inside your skull. The main question in this dissertation is "how" this lump containing primarily water and a little bit of fat gives rise to your consciousness and your behavior.

In 1906, Camillo Golgi from Italy and Santiago Ramón y Cajal from Spain shared a Nobel Prize for slicing up brains and staining them to reveal the 'brain processes' within. The two scientists were not friends, as the Italian believed the brain to constitute one large network, like a grid (the 'reticulum'), while the Spaniard claimed that it was made up of individual nerve cells (Rapport, 2005). Cajal was right: we now know that our brain contains roughly 86 billion neurons, each with some 1.000 to 10.000 connections to other neurons, the spatiotemporal activity of which forms the basis of all we experience, know, and do.

It is impossible to grasp the number of possible states the human brain could be in with all those neurons and connections: it is larger than the number of particles in the known universe. And yet, there is organization in that multitude. By evolutionary and developmental design, neurons involved in similar types of processing have often ended up next to each other (Barrett, 2012). For example, the primary visual cortex ('V1') is the outer layer at the back of the brain where visual inputs first reach the visual cortical system. In V1, neurons highly involved in detecting lines of certain orientation are organized in such a way that neurons preferring the same orientation are aligned in columns, while neighboring columns prefer 'neighboring' orientations (e.g. Hubel \& Wiesel, 1968). Moreover, neurons in these columns can prefer visual information presented to the left eye, or to the right eye, in a second dimension of organization (see Figure 1).

The distribution of perceptual tasks over the brain's many neurons is thus not random, which means it is graspable. It means the brain can be studied and, eventually, understood. In fact, that is the goal of the field of cognitive neuroscience; to link brain and mind, population activity and perception, networks and knowledge. If there are organization and principles behind brain function, they can be found.

\section{Linking Brain and Mind}

On September 13th 1848, Phineas Gage, foreman at railroad construction sites for Rutland and Burlington Railroad company, went to work at the tracks. His job was to blow up boulders that waylaid the tracks. To do this, he would drill a hole in the rock, fill it with blasting powder mixed with sand, set up a fuse and light it from a safe distance. Part of the job was to pound down the gunpowder mixture with a tamping 


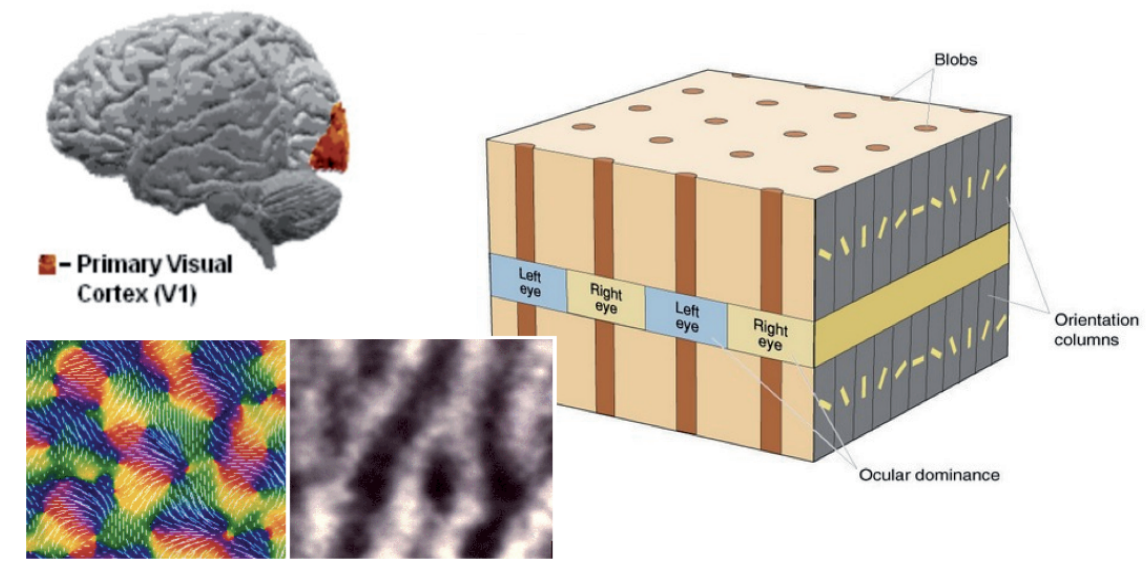

Figure 1: Organization in primary visual cortex

At the back of the brain, visual inputs first reach the cortical visual system at the primary visual cortex ('V1'). V1 is highly organized: neurons responding most strongly to edges of certain orientations are arranged in columns (color-coded with preferred orientations overlaid by white lines in lower left figure, and schematized in right figure). Orientation columns themselves are organized such that neighboring columns prefer 'neighboring' orientations. On top of this, there is a layer of organization concerning which eye's information certain neurons respond to (ocular dominance columns: black - white code for preferred eye in lower left figure, schematized in right figure). This organizational scheme repeats itself across the primary visual cortex.

(images from www.mcb.berkeley.edu/courses/mcb64/cortex.html, www.wikipedia.com, www.cs.utexas.edu/users/nn/web-pubs/sirosh/pvc.html, retrieved 10/2012).

iron; a 12-pound cylindrical iron rod. That Wednesday, the gunpowder ignited in an explosion, blasting the tamping iron upwards through Phineas Gage's left cheek, through his frontal brain, before it landed 25 meters further on. Gage reportedly got up almost straight away, able to walk and talk and ride a cart home. He did finally break down not too much later, but he survived, and lived another twelve years.

Phineas Gage was able to walk and talk again, meet again with his old friends, try again to resume work. Here, the story becomes muddled, as over the last centuryand-a-half the remarkable tale has been reported and re-reported with various degrees of freedom (Macmillan, 2000). Some reports claim that Gage became violent, fickle, unreliable and estranged. Some claim he abused his wife and children, although records show he never had any. Others state that he was perfectly able to hold a job, and made a remarkable social recovery. But all agree on one thing: he was changed. His old friends are famously quoted as saying, "Gage was no longer Gage". For a brain scientist, this case is as fascinating as it is tragic. We know clearly which part of the human brain was damaged ('lesioned'), and we have reports of what changed in personality and behaviour. The "astonishing hypothesis" (Crick, 1994) then, is that the neurons or 
processing in that particular brain region gave rise to aspects of Phineas Gage's personality.

No matter how much of Phineas Gage's anecdote is true, it does demonstrate the very foundation of cognitive neuroscience: to evaluate perception, cognition, emotion and behaviour, and link it to processes and/or areas in the brain. Patients that, like Phineas Gage, have localized brain lesions can display very concrete symptoms. Damage to a cluster called the fusiform face area results in a condition named prosopagnosia: faces are no longer recognised even though eyes, nose and mouth are clearly seen (e.g. Fox, Iaria, \& Barton, 2008), linking this region to face processing. If we sustain damage to the parietal cortex on the top-right of our brains, we may start neglecting things on our left (e.g. Corbetta \& Shulman, 2011). We may not even notice that we haven't touched the food on the left half of our plates, although we have polished off the right half of it and are still hungry. If the aforementioned primary visual cortex is damaged, we may not see anything in parts of our visual field. Yet, when prompted, we might correctly guess what is there (Weiskrantz, 1996).

While cognitive neuroscience could continue to develop and thrive on such interesting cases, it would remain fundamentally limited. Patients with such localized brain damage are relatively rare, and while the detailed perceptual, cognitive, behavioral effects of a brain lesion could be studied at length, the brain lesion itself could not be inspected until the patient passed away. Moreover, by that time especially, patients have had a long time to adapt to their condition which again changes the brain. Thus, for a long time healthy brains were inaccessible to neuroscientists. Besides, could we have temporarily removed our healthy brains from our skulls, what would we have seen in that uniform lump of pink? From this, it should become clear why cognitive neuroscience made such a giant leap forward when it became possible to actually investigate the brains of living, conscious, perceiving and behaving, healthy people.

\section{Levels...}

Fractals are mathematical functions that entail recurring patterns. When visualized, a fractal has a clear complex pattern on one level, and when zoomed in (or out), the same or a similarly complex pattern emerges again. One might consider each time the same pattern emerges a different level in the same mathematical reality. There are quite a few things in nature resembling fractals, such as snow crystals, certain leaves, and the Romanesco broccoli (see Figure 2). But more generally, the key to understanding many things is to consider them in terms of multiple levels constituting the same reality. Unlike in fractals, more often than not very different properties arise on different levels. $\mathrm{H} 2 \mathrm{O}$ molecules are not particularly wet, yet water is wet, although it is merely a collection of $\mathrm{H} 2 \mathrm{O}$ particles. A hurricane is a force of destruction, created by a lengthy process involving Coriolis forces and a positive feedback cycle of recurrent water evaporation and condensation, but on a smaller level a hurricane is just air and water. And who hasn't spent an evening admiring a flock of birds spiraling through the sky in complex and dynamic patterns. Such seemingly complex and intelligent behavior 'emerges' in the flock, even if the individual birds follow a limited set of simple rules. 
Unlike birds, human beings have dreams, thoughts and feelings. They have an extensive form of consciousness. Yet, these things are difficult to see when opening up a brain and looking at neurons. The brain may therefore also be considered as consisting in several levels. Molecules make up the organelles inside neurons, which in concert determine the neuron's state and activity, which in turn affects the activity of connected neurons which make up a neuronal column, which is part of a specialized region, which is connected to other regions in a network, which interacts with other networks, and so on. Somehow, this hierarchy gives rise to our behavior and consciousness. For the purposes of the current work, it is useful to distinguish the neuronal level (single or small populations of neurons), the regional level (functional areas in the brain such as aforementioned primary visual cortex), and the system-level (networks of regions, interacting with functional areas and each other). As we will see below, neuronal processes at all these levels may contribute to our visual consciousness.

\section{... and tools}

The advance in neuroscience since the time of Phineas Gage is that the brain can now be researched on all these levels. Even on the level of single neurons. Single-cell recording is usually done in animals, although occasionally also in humans when their brains have already been exposed for upcoming surgery. Recordings of single cells offer the advantages of both very high spatial resolution (= how spatially specific our measurements are) and temporal resolution (= how precise in time our observations can be) in the order of sub-milliseconds. Moreover, using similar equipment it is possible to stimulate single (or small groups of) neurons. Of course, since the brain works its magic by the patterned activity of constellations of cells across the brain - any such single-cell data only provide a small (though informative) piece of the puzzle.

To fully understand how the brain works we need to research it at higher levels as well. Just as we can measure or stimulate single neurons, we can measure or stimulate brain areas. We thus address the regional level of the brain. In general, brain measurement methods are referred to as 'brain imaging', and brain stimulation methods as 'brain interference'. For use in humans, noninvasive brain imaging methods include functional magnetic resonance imaging (fMRI: used in chapter III.4) and electro- or magnetoencephalography (EEG/MEG: used in chapter III.3). (Other imaging methods remain outside the scope of this dissertation.) In the category of brain interference methods that are painless, transient, and noninvasive (do not in any way enter or puncture the body), there are transcranial magnetic stimulation (TMS: used in Part II and chapter III.1) and more recently transcranial direct current stimulation (tDCS). We will conceptually introduce each of the methods applied in this dissertation, starting with the current mainstay of neuroimaging (Logothetis, 2008): fMRI. 

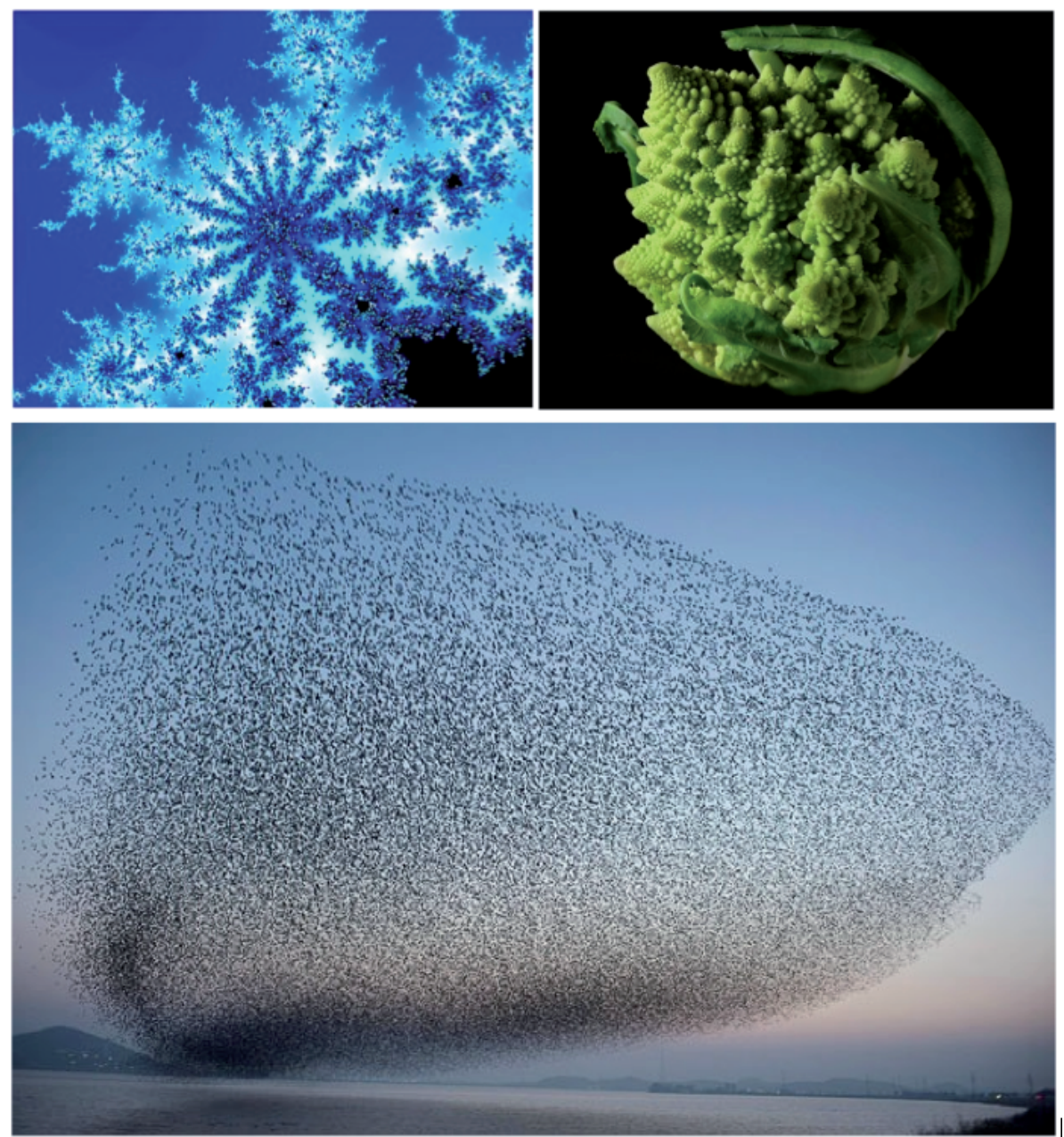

Figure 2: levels and emergence

Fractals (top-left) are mathematical functions that can be visualized in different levels, always showing identical or similar complexity. Nature sometimes approaches fractals, as in the broccoli shown on the top-right. But more generally, the concept of levels is incredibly useful in trying to understand nature. A key realization in the context of the current thesis is that sometimes, the same reality can have different properties on different levels. For instance, a flock of birds (bottom) consists of individual birds on one level, following a limited set of simple rules. But on a higher level, the flock as a whole displays complicated and beautiful emerging patterns. When looking at such a flock, our visual awareness of it may be a higher-order property, emerging from the concerted operations of the neurons of our visual system on a lower level.

(images from www.wikipedia.com, www.armedwithvisions.com/2011/07/17/deanetrwilderness-birds-of-extinction, www.coolmath.com/fractals/fractals_lesson.html, retrieved 10/2012) 


\section{Introduction to fMRI}

FMRI is a technique that in its current form was invented some twenty years ago. A steady rise in fMRI publications has brought the total to over 1500 fMRI research reports in 2011 alone (Smith, 2012). FMRI allows us to look with millimeter precision into the brain of everyday behaving, cognizant, conscious human beings, painlessly and with no adverse effects. Standard fMRI experiments evaluate the blood oxygenation level dependent (BOLD) signal. The most important limitation of fMRI is that this BOLD signal does not reflect neuronal activity directly (Logothetis, Pauls, Augath, Trinath, \& Oeltermann, 2001). Rather, it can measure throughout the brain how much oxygen is in the blood, which is informative since neuronal activity requires oxygen. Blood contains haemoglobin molecules, and haemoglobin loaded with oxygen has different magnetic properties from haemoglobin without oxygen: the latter distorts a magnetic field much more than the former. FMRI essentially sends radio pulses into each nook and cranny of the brain and detects the extent of such distortions, hence inferring how much oxygenated versus deoxygenated blood there is and deducing from this how much oxygen-requiring neuronal activity is taking place (for more details on physical and physiological mechanisms see Huettel, Song, \& McCarthy, 2004).

In recent years, fMRI experiments have begun to look beyond activity in regions to connectivity throughout the brain. By tracking activity over time in multiple brain regions, it is possible to identify networks throughout the brain that display similar (correlated) fluctuations - thus possibly engaged in similar tasks or processing. Using different analysis techniques, such as dynamic causal modeling (Friston, Harrison, \& Penny, 2003) or Granger causality analysis (Roebroeck, Formisano, \& Goebel, 2005), it has recently become possible to go even further than that, tracking directed interactions within such networks (applied in chapter III.4). This is a challenge, since fMRI has a limited temporal resolution in the order of seconds. Considering how little time it takes to read and process any of these words, it is clear that the brain works rather faster than that.

\section{Introduction to EEG/MEG}

EEG and MEG are more focused on the temporal domain, able to track brain activity with millisecond precision. Unfortunately these methods need to sacrifice some spatial resolution (in the order of centimeters). One might picture the type of neuron measured here as a short electrical wire. We noted that neurons are generally organized in 'populations', engaged in similar processing in close interaction. So, an active 'neuronal population' might be pictured as a set of parallel wires, or an electrical cable. When signals are sent through this 'cable', charged particles (ions) flow around it, reaching all the way through the skull to the scalp where this secondary current is picked up by sensitive electrodes. MEG measures almost the same signals, although it is sensitive to slightly different neuronal populations. The laws of physics dictate that whenever a current flows, a magnetic field exists perpendicularly to the current. While EEG picks up the current, MEG picks up this coexisting magnetic field (for more details on the 
physics or physiology of these methods, please see Hamalainen, Riita, Ilmoniemi, Knuutila, \& Lounasmaa, 1993; Niedermeyer \& da Silva, 2004).

EEG/MEG can be applied in several ways, to study the brain at several levels. For example, these methods can map out the response of a brain region (such as early visual cortex) to a repeated stimulus (such as a visual image). Previous work like this has suggested that such visual-evoked potentials (VEPs) begin to affect primary visual cortex from around 50-60 milliseconds after the onset of visual stimuli (e.g. Di Russo, Martinez, Sereno, Pitzalis, \& Hillyard, 2002) with a peak response around 90 milliseconds. This will be relevant in the context of 'TMS masking' paradigms discussed in Part II. Another application of EEG/MEG is the tracking of 'brain waves', or spontaneous oscillations in brain networks. As oscillations in the brain may be responsible for intra- and inter-regional communication (Buzsaki, 2006), the tracking of these oscillations can be meaningful at mid- to high-level brain investigations. For instance, the power and phase of alpha-oscillations (brain rhythms at around $10 \mathrm{~Hz}$ ) seems closely related to attention, and selection of which stimuli are consciously perceived (Mathewson, et al., 2011; Thut, Nietzel, Brandt, \& Pascual-Leone, 2006). This is the topic of chapter III.3.

In sum, although fMRI, EEG, and MEG differ in several ways, they have one thing in common: they correlate measured brain activity to perception, cognition or behavior. Brain interference methods including TMS add to this by 'taking control' of the brain processes. Thus, while in a brain imaging experiment a visual task is performed and resulting brain activity is measured, in a brain interference experiment the brain activity is manipulated and resulting visual task performance, or subjective conscious vision, is measured.

\section{Introduction to TMS}

TMS can manipulate brain activity in various ways, depending on the parameters of the magnetic stimulation. As noted above, basic laws of physics dictate that an electrical and magnetic field always co-occur. TMS exploits this principle. The currently most popular figure-8, or butterfly, coil employs two partly overlapping windings (coiled wires) (see Figure 3). A stimulator discharges a strong but brief electrical current through the windings. As the current flows, a magnetic field perpendicular to the coil windings arises. Since the current is so brief, the magnetic field exists very briefly as well, meaning it has a steep rise and decline. Such a rapidly changing magnetic field is also called a magnetic pulse. When applied to the head, the magnetic pulse effortlessly crosses the scalp and skull to reach the cortex. Just as an electrical current through matter induces a magnetic pulse, a magnetic pulse will induce an electrical field in matter, in this case brain matter. Since neuronal transmission and communication involve electrical charges and potentials, an induced changing electrical field can result in neurons firing (becoming active). If the cortical region thus stimulated was currently involved in spatiotemporally specific processing (e.g. processing a visual image), this undesirable wave of disorganized externally-induced neuronal activity disrupts the finely balanced internal symphony of organized neuronal activation patterns (not unlike 
disturbing a quietly whispered group discussion by suddenly shouting nonsense through a megaphone).

The magnetic field does decay rapidly with distance, so even with modern stimulators (reaching field strengths around 1.5-2.5 Tesla) only the superficial brain matter, i.e. cortex, is stimulated. Although this depends on stimulation intensity and cortical macroanatomy, as a rule-of-thumb a patch of cortex of about one square centimeter will be affected. This stimulation is momentary, and while single-neuron effects can in certain circumstances last for hundreds of milliseconds (Moliadze, Zhao, Eysel, \& Funke, 2003), for practical intents and purposes in cognitive neuroscience experimental settings, the duration of disruption by single pulses has been estimated to be around 510 milliseconds (e.g. Pascual-Leone, Walsh, \& Rothwell, 2000; Sack, 2006). So a single TMS pulse has an impressive spatial and temporal resolution. This can be exploited in paradigms using single-pulse TMS to chart the time course of functional relevance (crucial contributions) of a stimulated brain region for any task, cognition, or perception. In Part II, we apply this methodology to study functional relevance of early visual cortex for conscious (and potentially non-conscious) vision.

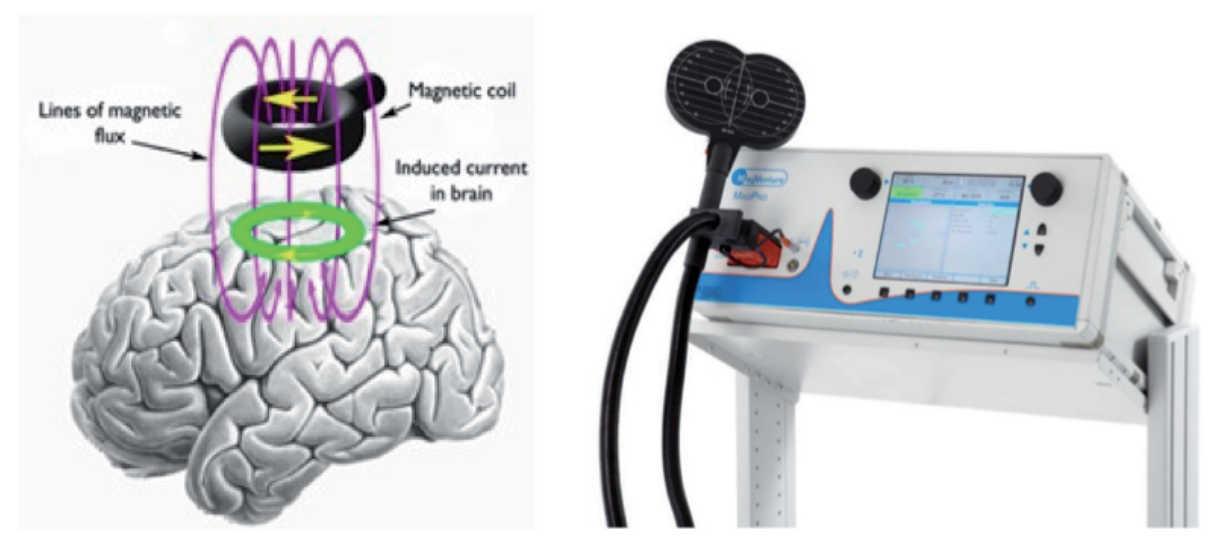

Figure 3: Transcranial Magnetic Stimulation

TMS involves 1) a strong electrical current through a coil, 2) magnetic field induction with perpendicular orientation, 3) induction of an electrical field in brain matter. Left: steps 1 to 3 schematically represented for a single-coil TMS device. Right: the MagPro TMS device with figure-8 (MC-B70) coil used in the work described here.

(images from http://www.med.upenn.edu/lcns/noninv.shtml,

http://www.magventure.com/default.aspx?pageid=196, retrieved 10/2012) 
Yet, the value of TMS as a research and clinical tool lies in its versatility. TMS can lead not only to momentary stimulations or disruptions but also to longer-lasting (though still temporary) changes in cortical excitability. Depending on stimulation

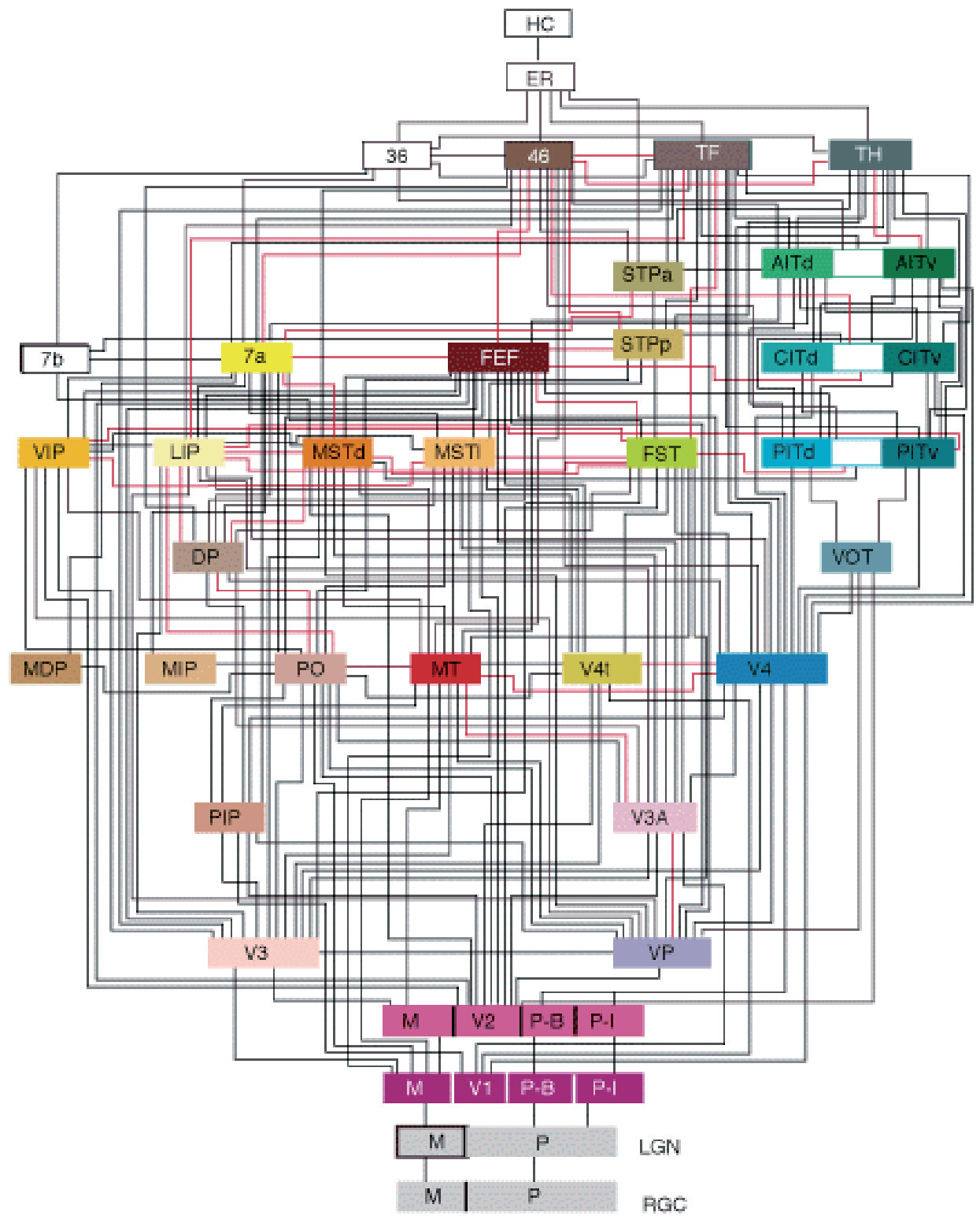

Figure 4: The visual hierarchy

The extended hierarchy of brain areas related to vision, and the connections between them.

Somehow, from this arise visual behavior and awareness. (from Felleman \& Essen, Cerebral Cortex, 1991). 
frequency, cortical regions can be made more, or less, excitable. Repetitive lowfrequency TMS (i.e. $1 \mathrm{~Hz}$ TMS) decreases excitability of the targeted region, resulting in what's been called a 'virtual lesion' (Pascual-Leone, et al., 2000). Repetitive highfrequency TMS (i.e. $10 \mathrm{~Hz}$ TMS) increases excitability, which means in certain conditions that cortical processing and therefore task performance can be improved. These longer-lasting TMS effects also imply that experiments can be designed in which TMS induces a (temporarily) lasting virtual lesion, after which behavioral effects can be measured 'offline' (i.e. without simultaneous TMS). This can be useful if the nonneural effects of TMS (i.e. sensory scalp stimulation, induced blinking, muscle twitching, or the 'clicking sound' accompanying each pulse) are uncomfortable or for other reasons interfere too much with task performance. We use this offline rTMS approach in chapter III.1.

\section{Psychophysics}

One of the exciting consequences of such interdisciplinary developments is that research fields become mutually informative. Based on improved knowledge of brain processes, new psychophysical studies can be designed to further probe brain dynamics using purely behavioral methods (Vanrullen \& Dubois, 2011). For instance, in chapter III.3 we aim to investigate the role of alpha oscillations in establishing conscious vision without actually measuring these oscillations. If this innovative methodology is successful, brain oscillations and their effects in various contexts can be manipulated and studied 'from the outside'. Valuable as brain imaging and interference tools are, they may therefore not always be required if psychophysical experiments are built on neuroscience-informed foundations and research questions. Also in Part I, we use purely psychophysical tools to probe mechanisms of neuronal competition underlying the selection of one or another image for access to visual awareness.

\section{Vision and Conscious Vision}

Having thus surveyed the methodological arsenal available to researchers interested in the brain and its relation to cognition, perception and behavior, we need to define the research topic we intend to apply these methods to. In the current dissertation, we focus on the establishment of conscious vision in the human brain.

\section{The distributed visual hierarchy}

Conscious vision is a process at the apex of the multiple levels of the visual brain. Neurons in the brain tend to fire in response to specific features of visual inputs (neuronal level). As noted above, fortunately neurons with similar preferences are often located together in regions. These regions constitute an extended and complex hierarchy of visual areas (regional level) (Felleman \& Van Essen, 1991). The basic premise of the visual hierarchy is that neurons at lower levels of the hierarchy preferably respond to 'simple' features. As we noted above, neurons in primary visual cortex ('V1') respond strongly to specifically one orientation, while others respond to another orientation. 
Upwards in the hierarchy, neurons begin to prefer simple shapes ('V2, V3'), colors ('V4'), motion ('V5'), until we reach areas preferring objects (lateral-occipital complex: LOC), faces (fusiform face area: FFA), 'places' such as buildings and other dwellings (parahippocampal place area: PPA), and further on in the temporal lobe neurons prefer object categories, tools, animals, and so on (see Figure 4).

To have succeeded in mapping these distributed processes is a major triumph for (neuro)science. But this mapped hierarchy leaves us with two questions. First, what if one sees a 'red car'? The 'redness' and the 'car' (and also the lines, contrasts, orientations, shapes making up the car) are apparently represented in separate visual brain areas. Yet, we see a 'red car', not separate 'redness' and 'car'. How this information is integrated is often referred to as the 'binding problem' (Revonsuo, 1999). A simple 'feedforward' approach to the visual hierarchy has difficulties resolving this issue, and so the current work addresses in Part II and Part III how the brain may reiteratively communicate within this hierarchy, and between these visual areas and other areas/systems in the brain.

The binding problem also relates directly to our second question. This question is: how do we SEE the red car? It is important to realize that this question has two parts, as we can split the concept of 'seeing' into at least two meanings. First, there is 'seeing' as in the processing of visual information in such a way that we can react to it. This form of 'seeing' is a form of seeing that an Iphone could perform as well: take in visual information, and use it in some way. The second sense of 'seeing' is the actual experience we have of it, the way something looks to us (Chalmers, 1996). Visual awareness, visual consciousness, conscious vision, we use these terms interchangeably throughout the thesis, to refer to this second aspect of seeing.

\section{The unified conscious visual experience}

The difference between the two aspects of seeing we refer to is not immediately obvious. But there are examples that demonstrate it clearly. For example, patients with lesions to the primary visual cortex sometimes have a condition called 'blindsight' (Weiskrantz, 1996). These patients do not experience (report) any subjective perception of stimuli presented to them in certain areas of their visual field, yet they relatively often will correctly discriminate, point to, or guess certain features of these stimuli. They can say that they might as well be guessing, having no idea at all what is presented to them, yet their brain clearly has enough information to make accurate judgments on the stimuli they don't consciously see.

Blindsight is rare. This second sense of seeing, the conscious visual experience, is something that fortunately most of us experience and enjoy all day every day. It is therefore of great scientific interest. How is the brain, with its constellation of 86 billion neurons, able to bring about such a unified and intimately familiar experience? As blindsight shows, being able to report on visual information does not always require conscious vision, so conscious vision is not identical to processing visual information. It is something additional that somehow emerges from the distributed activity across the visual brain. To truly understand the establishment of conscious vision, it will likely be necessary to approach this problem as one comprising processes 
at several levels of the brain. It is, after all, unlikely that there is one neuron, or one region, somewhere in the brain that determines whether or not you see something consciously or not.

There are some ideas on how the brain establishes specifically conscious vision. Lamme (Lamme, 2001, 2006; Lamme \& Roelfsema, 2000; Lamme, Super, Landman, Roelfsema, \& Spekreijse, 2000) has proposed that visual information first progresses through the visual hierarchy in a parallel, automatic, feedforward sweep, reaching all the way to parieto-frontal regions that allow us to react to the visual information. Then, the information is 'fed back' to earlier visual areas, to primary visual cortex, for more detailed and, eventually, conscious vision. Other theories, such as the global workspace theory (Baars, 1989; Baars \& Franklin, 2007) and its neuronal instantiation (Dehaene \& Naccache, 2001) suggest that visual information needs to reach frontal 'executive' and/or 'working memory' areas. These areas will make the visual information 'globally available' to other systems throughout the brain, and it is this global availability that constitutes visual consciousness. A third prominent theory on consciousness focuses on the complexity and connectivity that is required for conscious experience. This information-integration theory (Tononi, 2004) is in line with measurements showing that brain areas are more widely connected (connectivity) and display more differentiated responses to TMS-probes (complexity) when subjects are awake and conscious, than when they are in deep sleep and unconscious (Massimini, et al., 2005). These complex systems are distributed over the brain.

In our view, these theories are not mutually exclusive. In fact, they may be very complementary if one considers conscious vision to encompass functionally relevant processes, steps, and concurrent dynamics, at several levels of the brain. Simultaneously, in the endeavor to investigate and understand conscious vision in the brain, we believe it is useful to make a division between neural prerequisites, neural consequences, and actual neural substrates of conscious visual experience (see the Prologue). It seems possible that at some level(s) of the brain, processes correlated to conscious vision are required for the establishment of conscious vision, while processes at other level(s) may be identical to the experience, and processes at again other areas/level(s) may simply follow conscious experiences.

For all these reasons, in the current work we addressed the problem at several levels. We used a variety of methods to study vision, conscious vision, visual task performance, and visual cognition.

\section{Contents of this thesis}

The work described in this thesis aims to make several contributions to the understanding and study of conscious vision and its underlying dynamics. It thereby addresses the following (research) questions:

1) How can conscious vision be studied (and separated from other aspects of vision) in the human brain? (Prologue) 
2) How do low-level processes determine whether we do, or do not, become conscious of visual input? In other words: how do low-level processes co-determine whether/which visual information is 'selected' for visual consciousness? (Part I)

3) What are the dynamics in early visual cortex that are functionally relevant for conscious (and 'non-conscious') vision? What is the time course of involvement of early visual cortex? (Part II)

4) How do higher-level processes affect the processing of visual inputs? Do frontoparietal areas (implicated in conscious vision) top-down influence visual processing and the contents of conscious vision? (Part III)

The first question is a more conceptual one, which we address in the Prologue. Here, we discuss further how conscious vision can be studied in the human brain, which paradigms can usefully be applied, and propose a division into several 'kinds' of neural correlates of consciousness.

In subsequent chapters we follow-up on these proposals, studying the establishment of conscious vision in the brain by applying a range of methods, including psychophysics, TMS, fMRI, and MEG, focusing on a range of potentially involved brain processes. These we subdivided into three main 'Parts' to maintain an overview.

\section{Thesis outline}

In Part I, we present a potentially new visual phenomenon, referred to as the 'disrupted rivalry effect'. This effect, obtained in an experimental setup of 'binocular rivalry', involves the failure of the visual system to select for conscious vision a salient image that is presented to participants. Although this image is clearly on screen and has no clear distractors or competition for visual awareness, the novel experimental manipulation results in participants failing to perceive it consciously for seconds on end. In chapter I.1 we establish this effect, in chapters I.2, I.3, and I.4 we develop and test a proposed model of its underlying neuronal dynamics.

In Part II, we specifically aim to disrupt conscious vision and/or potentially non-conscious visual processing, in early visual cortex using single-pulse TMS. Early visual cortex, particularly $\mathrm{V} 1$, has been suggested, and by others contested, to be involved in conscious vision (see above; Lamme's theory of conscious vision). By further developing a traditional paradigm called the 'TMS masking' paradigm in a series of exploratory experiments, we aimed to chart the time course and several stages of functional relevance of this region in the establishment of vision. In chapter II.1 we suggest that 'sensitive TMS masking paradigms' may yield new results within a 'classical masking window'. In chapter II.2 we present two experiments focusing on the influence of different kinds of stimuli and tasks on masking results. In chapters II.3 and II.4 we demonstrate that TMS pulses can disrupt conscious vision not only when administered after the presentation of visual stimuli, but also when applied before visual information even appears on screen. In chapter II.5 we review how these findings, and other recent TMS masking experiments, suggest that the 'classical' masking effect may hold further insights, and what all these findings mean for the various stages of early 
visual cortex involvement in (conscious) vision. Specifically, throughout these chapters we address and test the theory that conscious vision requires recurrent signals to early visual cortex.

In Part III, we investigate the influence of higher-order visual areas on conscious vision and visual cognition. In chapter III.1 we address the hypothesis that frontoparietal areas directly influence the contents of conscious vision (see above: global workspace theory of conscious vision), again using a bistable perception paradigm and now applying inhibitory rTMS. One of the conclusions here is based on a null result, and in chapter III.2 we present a conceptual contribution on when null results in TMS can and cannot be interpreted meaningfully. In chapter III.3 we address a different higher-level brain process hypothesized to determine the selection of visual information for conscious vision, following up on the role of alpha oscillations. We used psychophysical methods to 'entrain' alpha oscillations, evaluating their influence on visual task performance and relating these results to MEG measurements. While chapter III.1 addressed frontal influences on vision, and chapter III.3 considered parieto-occipital influences, chapter III.4 also evaluates top-down influences on successful visual processing, in an fMRI connectivity study on frontoparietal involvement in visual cognition. 


\section{References}

Baars, B. J. (1989). A Cognitive Theory of Consciousness. New York: Cambridge University Press.

Baars, B. J., \& Franklin, S. (2007). An architectural model of conscious and unconscious brain functions: Global Workspace Theory and IDA. Neural Netw, 20(9), 955-961.

Barrett, H. C. (2012). A hierarchical model of the evolution of human brain specializations. Proc Natl Acad Sci U S A, 109 Suppl 1, 10733-10740.

Buzsaki, G. (2006). Rhythms of the Brain. New York: Oxford University Press.

Chalmers, D. J. (1996). The Conscious Mind: in search of a fundamental theory. Oxford: Oxford University Press.

Corbetta, M., \& Shulman, G. L. (2011). Spatial neglect and attention networks. Annu Rev Neurosci, 34, 569-599.

Crick, F. (1994). the astonishing hypothesis: the scientific search for the soul. New York: Touchstone.

Dehaene, S., \& Naccache, L. (2001). Towards a cognitive neuroscience of consciousness: basic evidence and a workspace framework. Cognition, 79(1-2), 1-37.

Di Russo, F., Martinez, A., Sereno, M. I., Pitzalis, S., \& Hillyard, S. A. (2002). Cortical sources of the early components of the visual evoked potential. Hum Brain Mapp, 15(2), 95-111.

Felleman, D. J., \& Van Essen, D. C. (1991). Distributed hierarchical processing in the primate cerebral cortex. Cereb Cortex, 1(1), 1-47.

Fox, C. J., Iaria, G., \& Barton, J. J. (2008). Disconnection in prosopagnosia and face processing. Cortex, 44(8), 996-1009.

Friston, K. J., Harrison, L., \& Penny, W. (2003). Dynamic causal modelling. Neuroimage, 19(4), 1273-1302.

Hamalainen, M., Riita, H., Ilmoniemi, R., Knuutila, J., \& Lounasmaa, O. (1993). Magnetoencephalography-theory, instrumentation, and applications to noninvasive studies of the working human brain. Reviews of Modern Physics, 65(2), 414-497.

Hubel, D. H., \& Wiesel, T. N. (1968). Receptive fields and functional architecture of monkey striate cortex. J Physiol, 195(1), 215-243.

Huettel, S. A., Song, A. W., \& McCarthy, G. (2004). Functional Magnetic Resonance Imaging. Sunderland, MA: Sinauer Associates, Inc.

Lamme, V. A. (2001). Blindsight: the role of feedforward and feedback corticocortical connections. Acta psychologica, 107(1-3), 209-228.

Lamme, V. A. (2006). Zap! Magnetic tricks on conscious and unconscious vision. Trends Cogn Sci, 10(5), 193-195.

Lamme, V. A., \& Roelfsema, P. R. (2000). The distinct modes of vision offered by feedforward and recurrent processing. Trends Neurosci, 23(11), 571-579.

Lamme, V. A., Super, H., Landman, R., Roelfsema, P. R., \& Spekreijse, H. (2000). The role of primary visual cortex (V1) in visual awareness. Vision research, 40(10-12), $1507-1521$ 
Logothetis, N. K. (2008). What we can do and what we cannot do with fMRI. Nature, 453(7197), 869-878.

Logothetis, N. K., Pauls, J., Augath, M., Trinath, T., \& Oeltermann, A. (2001). Neurophysiological investigation of the basis of the fMRI signal. Nature, 412(6843), 150-157.

Macmillan, M. (2000). An Odd Kind of Fame: The MIT Press.

Massimini, M., Ferrarelli, F., Huber, R., Esser, S. K., Singh, H., \& Tononi, G. (2005). Breakdown of cortical effective connectivity during sleep. Science, 309(5744), 22282232.

Mathewson, K. E., Lleras, A., Beck, D. M., Fabiani, M., Ro, T., \& Gratton, G. (2011). Pulsed out of awareness: EEG alpha oscillations represent a pulsed-inhibition of ongoing cortical processing. Front Psychol, 2, 99.

Moliadze, V., Zhao, Y., Eysel, U., \& Funke, K. (2003). Effect of transcranial magnetic stimulation on single-unit activity in the cat primary visual cortex. $J$ Physiol, 553(Pt 2), 665-679.

Niedermeyer, E., \& da Silva, F. L. (2004). Electroencephalography: Basic Principles, Clinical Applications, and Related Fields: Lippincot Williams \& Wilkins.

Pascual-Leone, A., Walsh, V., \& Rothwell, J. (2000). Transcranial magnetic stimulation in cognitive neuroscience--virtual lesion, chronometry, and functional connectivity. Curr Opin Neurobiol, 10(2), 232-237.

Rapport, R. (2005). Nerve Endings. New York: W.W. Norton \& Company, Inc.

Revonsuo, A. (1999). Binding and the phenomenal unity of consciousness. Conscious Cogn, 8(2), 173-185.

Roebroeck, A., Formisano, E., \& Goebel, R. (2005). Mapping directed influence over the brain using Granger causality and fMRI. Neuroimage, 25(1), 230-242.

Sack, A. T. (2006). Transcranial magnetic stimulation, causal structure-function mapping and networks of functional relevance. Curr Opin Neurobiol, 16(5), 593-599.

Smith, K. (2012). Brain imaging: fMRI 2.0. Nature, 484(7392), 24-26.

Thut, G., Nietzel, A., Brandt, S. A., \& Pascual-Leone, A. (2006). Alpha-band electroencephalographic activity over occipital cortex indexes visuospatial attention bias and predicts visual target detection. J Neurosci, 26(37), 9494-9502.

Tononi, G. (2004). An information integration theory of consciousness. BMC Neurosci, 5,42 .

Vanrullen, R., \& Dubois, J. (2011). The psychophysics of brain rhythms. Front Psychol, 2, 203.

Weiskrantz, L. (1996). Blindsight revisited. Curr Opin Neurobiol, 6(2), 215-220. 
Prologue 



\title{
P.1
}

\section{The "Correlates" in Neural Correlates}

\author{
of Consciousness
}

\author{
Related publication(s) \\ De Graaf, TA, Hsieh, JP*, Sack, AT. (2012). The 'correlates' in neural correlates of \\ consciousness. Neurosci Biobehav Rev, 36: 191-7. \\ De Graaf, TA, Sack, AT. On the various neural correlates of consciousness: can they be \\ distinguished? In upcoming volume of series Advances in Consciousness Research, ed. \\ Miller, S., John Benjamins, Amsterdam, the Netherlands \\ * Po-Jang Hsieh \\ 1) Department of Brain and Cognitive Sciences, McGovern Institute, Massachusetts \\ Institute of Technology, Cambridge, MA 02139, USA \\ 2) Duke-NUS Graduate Medical School, Singapore 169857, Singapore
}




\begin{abstract}
What sorts of brain processes covary with consciousness? We suggest that neural correlates of consciousness (NCCs) include neural substrates (actual conscious experience representation in the brain), neural prerequisites and neural consequences of a conscious experience. In a conventional correlative paradigm, these three NCCs are principally indistinguishable. However a multi-pronged research program might begin to disentangle them. We here integrate our ideas with other recent considerations on the various components of the empirically defined NCC, and propose strategies to address the challenges faced by the problem of three NCCs. We conclude that while this problem is important and difficult, it is not destructive. The NCC research program still has much to contribute.
\end{abstract}




\section{Introduction}

Over centuries, consciousness has been an obvious truth, a non-topic, unfashionable, the holy grail of scientific endeavor, and at times flat-out denied. Today, though 'consciousness' elicits the occasional grimace in researchers who consider it too vague a concept, it seems acceptable as a research topic. The burgeoning of empirical consciousness research may be related to the advent of new brain research tools. The subfield that aims to understand how consciousness arises from biological processes largely focuses on the brain, looking for the 'neural correlates of consciousness' or 'NCCs'. In short, NCC research tries to relate changes in brain processes to changes in consciousness.

Exactly because 'consciousness' is such a vague concept, it is crucial for the future of this research community that terms, topics, and findings are analyzed, operationalized, and schematized as clearly as possible. Many great minds have turned to this issue (Chalmers, 2000; Chalmers, 1996; Crick, 1994; Edelman and Tononi, 2000; Koch, 2004), contributing to the questions of 'what is consciousness', 'can we find consciousness in the brain', and 'if we find an NCC, what does it mean'?

To the latter question, several independent contributions have recently been made. We here discuss how different 'types' of processes may correlate to conscious vision, which means that different types of NCC exist (de Graaf, Hsieh \& Sac, 2012). Independently, a different group (Aru, Bachmann et al., 2012) has come to similar distinctions and related ideas (based in part on (Bachmann, 2009; Melloni and Singer, 2010). Miller $(2001,2007)$ moreover provided an extensive analysis on how to interpret an NCC, noting that not every empirically-identified neural correlate of consciousness is necessarily constitutive of that conscious state. He thus made a distinction between the neural correlates of consciousness and the neural constitution of consciousness. Other philosophically inclined contributors have debated how NCCs should be regarded in the grand scheme of understanding consciousness (Hohwy, 2009; Neisser, 2012; Noë and Thompson, 2004).

What these various contributors appear to agree on, as we will see below, is that there is indeed a (thusfar) largely neglected problem for NCC research that needs confronting. They are not (yet) so agreed on how and whether we can resolve it. Our view of the problem is that there is not one NCC but there are actually three, generally lumped together. Here, we aim to compare and synthesize our previous analysis with aforementioned contributions to provide an updated, empirically-focused analysis. With that goal in mind, we first need to clarify what we mean by 'consciousness' in the current context. We then briefly cover the basic methodology of the NCC research field to be clear on what types of findings we refer to, to set the stage for our central question: what does it mean if brain process $\mathrm{B}$ consistently covaries with conscious event $\mathrm{C}$ ? We will conclude with a discussion of the various strategies proposed by us and others to confront the central problem of multiple NCCs. As we are not philosophers, our discussion is from an empirical perspective even if the problem is partly conceptual. 


\section{What is 'consciousness'?}

Since there is nothing as unfruitful as a debate in which contributors inadvertently speak of different things, 'consciousness' needs to be clearly and unambiguously delineated and defined in any article or discussion considering it. In our view, it is useful to distinguish four notions of 'consciousness', all valid as types, kinds, of aspects of consciousness, and all worthy of and susceptible to scientific inquiry in their own right, but substantially different from each other conceptually. We call these self-awareness, higher-order awareness, medical awareness (= state-consciousness), and consciousness as experience (= content-consciousness). This is not the venue to discuss extensively the relation of these notions to all other published taxonomies, but it is important to briefly explain them so that it is clear which 'type' of consciousness is the one we focus on in the remainder of the chapter. In general, we use 'consciousness' and 'awareness' interchangeably.

Self-awareness, or self-consciousness, refers to all mental and cognitive processes that allow us to identify ourselves as an individual with mind and body, a subject in a world otherwise filled with other individuals and other things. It is the 'you' that you perceive as being at the center of your experiential world, the coordination and continuation of the essence of your personality, the one in control of your body and decisions (e.g. Blanke, 2012). Research about self-consciousness focuses on which brain regions host your 'sense of self' or your personality, how you have a sense of 'agency' when your body moves or performs tasks, or which animals (or humans from which age onward) recognize themselves in a mirror (e.g. see Gusnard, 2005).

Higher-order awareness refers to those mental and cognitive aspects that allow us to reflect and act nonimpulsively. It constitutes many functions that separate us from other animals: the ability to consider things in our minds, such as past, present and future. We feel that discussions about whether consciousness requires language (because otherwise you cannot 'think' as we think) are in this general realm of 'consciousness' as a concept. It is a notion of consciousness similar to for example 'reflective consciousness' (e.g. Edelman and Tononi, 2000), defined in Oxford English Dictionary as 'the recognition by the thinking subject of its own acts or affections'. It may be that much of the folk psychology conception of what 'consciousness' ought to refer to lies in this realm.

Medical awareness we have so dubbed because the corner of research that focuses on this type of awareness is often occupied by medical scientists (e.g. Gosseries et al., 2011). The question here is what (in the brain) is necessary for having any 'consciousness' as all? What determines whether we are in a conscious state, and what this state entails? Largely similar to the notion of 'state consciousness' (e.g. Hohwy, 2009), this aspect of consciousness is studied in experiments comparing deep sleep versus wakefulness (Massimini, Ferrarelli et al., 2005), comatose versus healthy subjects (Owen, Coleman et al., 2006), and drugged versus sober participants (Ferrarelli, Massimini et al., 2010). There is some potential overlap here between the study of medical/state consciousness and consciousness as experience/content consciousness, because the state a subject is in (e.g. sober vs drugged) may affect the phenomenal quality of any conscious experience had. But on the whole, medical 
awareness - being in a state of consciousness - is a precondition for the last aspect of consciousness we delineate here: consciousness as experience. You must be conscious (in a conscious state) in order to have any conscious experiences.

Consciousness as experience is the last and here most important type, kind, category or perspective on consciousness. It is at the same time the most difficult and the easiest notion of consciousness. In your mind right now, you experience different things over time. In the sense of 'medical awareness' or 'state-consciousness' you are constantly in a state of consciousness (if you manage to stay awake), but the contents of conscious experience change. Whatever is experienced at any one point in time, in any modality or any intensity, is what is - at that point in time - consciousness as experience. Research questions or paradigms in this category ask 'what (in the brain) defines whether you have this conscious experience or that conscious experience?' or 'what (in the brain) defines whether you do or do not have a particular conscious experience'. This notion of consciousness is the easiest in the sense that everyone at any time has a prime example in their own current experience. It is the most difficult because it entails the 'hard problem' (Chalmers, 1996) ${ }^{1}$.

Block (2005) distinguishes phenomenal and access consciousness. Phenomenal consciousness IS the experience itself: it is the qualitative aspect of an experience, the 'what it is like' to have that experience, the 'quale' (plural 'qualia'). The hard problem involves the questions: 'how is it possible that such subjective, qualitative experiences arise from mere neurons?' or 'why does an information processing machine like an organism have, or need, any conscious experience if all the same functions could conceivably be performed without the qualia?' or 'why is there this particular neuralphenomenal relationship and not some other?' (Chalmers, 1996). The information processing aspect here, the processes in the brain allowing a piece of information to be experienced consciously, e.g. involving global availability of that item of information to widespread systems across the brain (Dehaene and Naccache, 2001; Tononi, 2004), is called access consciousness. 'Consciousness as experience' in the current taxonomy, for most intents and purposes, can be said to cover both. In most cases, phenomenal and access consciousness will, after all, coincide. But it should be kept in mind that, when studying consciousness as experience, researchers generally want to find out what underlies phenomenal consciousness. So, if phenomenal consciousness and access consciousness turn out NOT to be implemented identically in the brain, 'consciousness as experience' in our framework would refer primarily to phenomenal consciousness.

\footnotetext{
1 There are some tricky confounding of elements of 'self-consciousness' and 'consciousness as experience'. You may experience, when focusing on it, a 'sense of self', and also a 'sense of control or agency' when doing something. It is important to mention that any such experiences of being 'a self' in this sense belong with 'consciousness as experience'. Also confusing is the idea of a 'pre-reflective self-consciousness' that is always present, facilitating any and all conscious experience as being 'in the eye of the beholder', making it your experience (Gallagher and Zahavi, 2010; Noë and Thompson, 2004). Again, in as far as this constitutes a mental process that is not directly experienced, it would fall in the category of analysis and study we call 'self-consciousness', but as soon as it is part of conscious experience directly it is 'consciousness as experience'.
} 


\section{NCC research}

\section{What is an NCC?}

One of the most highly-cited papers in the NCC literature is one published by David Chalmers in a volume about the NCC research field (edited by T. Metzinger 2002). It involves a delineation of what exactly is meant by an NCC:

An NCC is a minimal neural system $N$ such that there is a mapping from states of $N$ to states of consciousness, where a given state of $N$ is sufficient, under conditions $C$, for the corresponding state of consciousness. (Chalmers, 2000, p. 31)

This definition is widely accepted, although it does raise discussion (Neisser, 2012; Noë and Thompson, 2004). This definition applies primarily to research about content-consciousness, resting on the contrastive analysis (Aru, Bachmann et al., 2012; Baars, 1989; see below). The "minimal" in the definition above is important in the current context. It acknowledges that several processes (e.g. regional activities, frequency-specific oscillation power modulations, particular connectivities, and so on) in the brain might correlate to a conscious experience in a given paradigm, without all of these processes being required for the conscious experience (or even directly involved). This foreshadows the main point of the current chapter. Although by referring strictly to brain processes correlating to conscious experience we might avoid a quagmire of metaphysical issues, this may in the end remain unsatisfactory. Eventually we will want to understand how something works, not just record a collection of correlates without further interpretation.

\section{How do you find an NCC?}

But to proceed, we first need to obtain good NCCs. As cognitive neuroscientists, we have several tools at our disposal (see General Introduction). Brain imaging methodology revolves around the contrastive method: a subject's brain activity is tracked in an experimental condition that involves the mental function/behavior of interest, and contrasted with the activity in a control condition that mimics the experimental condition in all respects but the process of interest. Motion processing area hMT/V5 is easily identified by presenting moving dots and stationary dots and contrasting activity throughout the brain between these two conditions. But when trying to study the conscious experience of motion, a special problem arises. Moving dots and stationary dots will definitely result in different conscious experiences, but will also result in various different unconscious processes. Conscious and unconscious brain activity will fully coincide in this experiment. That is why, in NCC research, it is necessary to do one of two things: 1) manipulate the conscious percept without affecting unconscious processes, or 2) manipulate the unconscious processes without 
affecting the conscious percept. This allows one to separate the neural events in the brain underlying conscious and unconscious processing. In practice, although not strictly valid, manipulating (or not) the 'unconscious processes' is approximated by manipulating (or not) the stimuli and stimulation parameters. We have categorized and outlined the various paradigms that implement this strategy.

\section{Paradigms for the NCC researcher}

While potentially not exhaustive, we have found the following outline of methods surprisingly useful for maintaining an overview of NCC methodology. It delineates three major categories: illusions, multistable perception, and ON-OFF paradigms.

1) In illusion paradigms, researchers manipulate the conscious percept while affecting only minimally, or not at all, the stimulation.

1.1) One subclass of illusions involves illusory features. For example, in the Kanisza triangle, three pacman-like shapes can create the illusion of contours forming a triangle. A control condition involves the same pacman elements, but not illusory contours. A classic study used such a paradigm to find that V2 cells fire already in response to illusory contours (von der Heydt, Peterhans et al., 1984).

1.2) A second subclass of illusions involves hallucinations. For example, schizophrenic patients with auditory hallucinations were found to display activity in primary auditory cortex when hearing voices (conscious percept change) that were not there (no stimulation change) (Dierks, Linden et al., 1999).

1.3) Like illusory contours, there are "illusory textures" in the well-known process of filling-in (e.g. Weil and Rees, 2011), which could be tracked over time.

1.4) the fourth subclass of illusions involves after-images. When a stimulus is adapted to and removed, stimulation ceases, but a form of conscious percept remains and fades over time (Zaidi, Ennis et al., 2012).

2) Multistable paradigms are something of a staple item in NCC research. A constant stimulus gives rise to alternating conscious experiences over time (e.g. (Blake, 2001; Fox, 1991; Howard and Rogers, 1995; Kim and Blake, 2005; Kleinschmidt, Buchel et al., 1998; Levelt, 1965; Wheatstone, 1838; see Part I for extended discussion).

2.1) In binocular rivalry, one eye is presented with one image (e.g. a face), and the other eye with a second image (e.g. a house). Conscious experience alternates between the two images despite constant visual input, and the subject indicates when a face or house is perceived by pressing either of two buttons. Important early imaging work examined brain activity related to these switches to frontoparietal areas (Lumer, Friston et al., 1998; Lumer and Rees, 1999) and showed that activity in house- and face-specific regions (Tong, Nakayama et al., 1998) covaried with the conscious percept.

2.2) Monocular rivalry is a variant of this paradigm in which two representations presented to the same eye are 'dominant' alternatively (Breese, 1899), as is

2.3) pattern rivalry, where two images are presented to one eye each, but swapped between eyes in rapid alternation (Logothetis, Leopold et al., 1996). In the latter, the fact that conscious percept changes over time at its own pace (as opposed to strictly 
following the presentation alternation rates) shows that to some extent the 'higherorder' representations of the images can rival (Tong, Meng et al., 2006).

2.4) Ambiguous images yield the same conclusion: the Necker cube, Rubin's face-vase, the famous duck-rabbit, can all be perceived in two ways, but never simultaneously. Conscious perception changes, the stimulation remains constant.

3) $\mathrm{ON}-\mathrm{OFF}$ paradigms contrast conditions in which an image is perceived $(\mathrm{ON})$ versus is not perceived (OFF).

3.1) In 'weak ON-OFF' paradigms, this requires a small change in the stimulation parameters. For example in a classic masking study by Dehaene et al (Dehaene, Naccache et al., 2001), there was a visible condition (ON) in which a word was flanked by masks but with an intermittent blank period of $71 \mathrm{~ms}$ (no masking) and an invisible condition (OFF) in which there was no such blank period (masking). Comparing the $\mathrm{ON}$ and OFF conditions revealed, interestingly, that frontoparietal areas displayed significant activity specifically in the ON condition: an NCC.

3.2) Useful as weak ON-OFF paradigms are, 'strong ON-OFF' paradigms are slightly superior, since there is no change in the stimulus parameters at all, thus taking even more 'unconscious' processes out of the equation. FMRI research often needs to implement weak ON-OFF paradigms, in order to use powerful block designs. Imaging methods with higher temporal resolution can opt for strong ON-OFF paradigms however, in which the stimulation parameters are calibrated such that on some trials, an image (or other type of stimulus) is perceived, and on other times not. Subjects indicate on every trial whether they saw the stimulus or not, and ON and OFF condition are assigned post-hoc. For examples, see (Busch, Dubois et al., 2009; Mathewson, Gratton et al., 2009).

\section{The obtained NCC: what does it mean?}

Now that we have obtained our NCC, are we done? Philosophically oriented (Hohwy, 2009; Miller, 2007; Neisser, 2012; Noë and Thompson, 2004) and empirically orientated (Aru, Bachmann et al., 2012; Bachmann, 2009; de Graaf, Hsieh et al., 2012; Melloni and Singer, 2010; Miller, 2001, 2007) contributors have recently taken the next step. Neisser (2012), Hohwy (2009), and Noë and Thompson (2004) have argued that obtaining the NCC will only deliver part of the story. It is not valid to simply conclude that, whatever the NCC is, the conscious experience 'happens there' (Hohwy, 2009). The NCC program is able to provide a piece of the puzzle, but each NCC as resulting from one of the paradigms outlined above should be reinterpreted as one causal link in a complex sequence of actions (e.g Noë and Thompson, 2004; Neisser, 2012). The background conditions that allow this NCC to make a difference to our conscious perception are not included in any given NCC result, although they significantly affect how much, and what sort of, meaning we should ascribe to the NCC obtained.

But aside from these caveats to the NCC methodology, there is an additional question. What role, or function, does the NCC we have obtained play? Have we now found the instantiation (or what Miller refers to as the 'constitution') of the conscious experience in the brain? Not really: in our view any obtained NCC, whichever form it 
takes, in whichever correlative paradigm it was found, can have, at least, one of three different functional roles ${ }^{2}$.

\section{The three 'correlates' in neural correlates of consciousness}

Any NCC can reflect neural prerequisites for the conscious experience, may be (part of) the neural consequences of the conscious experience, or may be (part of) the neural substrate of the conscious experience itself. In what follows, by 'NCC' we will mean any neural event, neural process, chemical concentration, activity change, or whatever else can happen in the brain, that correlates consistently with a conscious experience and thus can come out of the sorts of NCC paradigms and experiments outlined above.

\section{Neural prerequisites of consciousness}

It may be that the obtained NCC was necessary for this conscious experience (' $\mathrm{C}$ ') to arise, ceteris paribus. It is, however, NOT itself the neural substrate of C. Given the state of all other processes in the brain, if this neural event did not occur, $\mathrm{C}$ would not have existed (in that form). 'Necessary' is a tricky term in consciousness talk, because it can be taken to suggest that specifically THAT neural event must take place for the conscious experience to arise. We do not claim as much. Perhaps a different neural event could also lead to $\mathrm{C}$ (i.e. give rise to the neural event that was the substrate), but it didn't: in this case it was the NCC we found that did the job.

Conceptually, neural prerequisites could take several forms. Content-specific prerequisites would be neural events necessary for the establishment of a particular conscious experience. For example, Crick and Koch have argued that neurons in primary visual cortex cannot be part of the (true) NCC (Crick and Koch, 1995). Yet, studies have reported a correlation between activity in primary visual cortex and conscious experience (Tong, 2003) and abolishment of primary visual cortex leads to a complete lack of visual consciousness (Weiskrantz, 1996). So, does consciousness reside in primary visual cortex or not? Silvanto (2008) tries to resolve this debate by arguing that processes in primary visual cortex might be crucial for consciousness to

\footnotetext{
${ }^{2}$ Various NCC contributors have acknowledged that NCC facts are not so simple. Miller (2007, p.162) provides a nice overview of this realization in previous work. For example, according to Crick (1994; p.218): 'it does not follow that these particular neurons are the real seat of awareness. They may by their firing, influence other neurons ... that are the true correlates of awareness' (see also Crick and Koch, 1998; Logothetis, 1998; Revonsuo, 2000, 2001). Miller (2007) points out though, that unequivocal determination of these actual constituents ("true correlates") of consciousness may in the end be impossible. In fact, for this problem "even an impression of a strategic solution is not yet apparent" (Miller, 2007; p161). However, that does not imply that substantial scientific progress in this arena cannot be made (Aru, Bachmann et al., 2012; de Graaf, Hsieh et al., 2012; Miller, 2001; 2007, Revonsuo, 2001).
} 
arise, but that consciousness itself resides elsewhere in the brain. This suggests in our framework that processes in primary visual cortex are neural prerequisites, but not substrates, of consciousness: certainly an NCC, but not the locus of conscious experience. The fact that a particular conscious experience requires a particular constellation of activity in V1 (e.g. a horizontal versus a vertical bar) suggests that V1 activity is a content-specific prerequisite for consciousness.

Connectivity between reticular formation and precuneus may be necessary for any conscious experience to arise (Silva, Alacoque et al., 2010). Such an NCC is an example of a content-invariant prerequisite. There is a range of possibilities in-between content-specific and content-invariant prerequisites for consciousness. For example, there could be such a thing as modality-specific prerequisites.

\section{Neural consequences of consciousness}

By neural consequences, we mean that an NCC was a result of the conscious experience. Because the conscious experience was there, this activation arose. It is not itself part of the experience, it is not necessary for the experience, yet it always coincides with the experience. There can be two forms of this (de Graaf et al., 2012).

Imagine a binocular rivalry study with two images: a rose and a house. Whenever we consciously experience the visual image of a rose, we are reminded of our deceased grandmother, who grew roses in her garden. We develop a range of (nonvisual) emotions and associations, all covarying with the (visual) conscious roseexperience, some conscious and others not. None of these are relevant to the conscious experience of interest. Yet, their neural underpinnings will be an NCC, since they always follow the experience of a rose. This exemplifies the content-specific (or stimulus-specific) form of neural consequences. In this example, the grandmotherassociation is also participant-specific. Looking at different participants would eliminate for the most part this NCC. But one can imagine content-specific yet participant-invariant neural consequences quite easily, all it requires is for most participants to have a similar association or value-response to the content in question. Such consequences could be countered using different kinds of stimuli.

That will not resolve content-invariant consequences, however. A conscious experience, of any kind, may consistently trigger specific neural events. Think of response preparation, effects on arousal/vigilance, attention capture, or just a general "oh, I just had an experience" effect. Aru et al (2012) point out that consequences, rather than useless and epiphenomenal, are a logical consequence of conscious experience, if one assigns any functionality to consciousness. Citing Seth (2009), they note that many theories of consciousness do in fact confer concrete functions to conscious experience, including novel, intentional behavior and enabling long-term memory. So, while troublesome in the context of the NCC program, these neural consequences may actually be highly relevant to the organism. 


\section{Neural substrates of consciousness}

There is a third functional role for any given NCC, and it is the one we are actually most interested in: the NCC may be the neural instantiation, neural constitution (Miller, 2001, 2007), or neural substrate (de Graaf, Hsieh et al., 2012), of the experience. The $\mathrm{NCC}$ is directly underlying the experience, not a result and not a precondition and is the minimal neural activity that is sufficient for a specific conscious percept, ceteris paribus (Chalmers, 2000; Koch, 2004). Considering the definition of Chalmers, this is to an extent the 'real' NCC, or the aforementioned 'true' correlates (footnote 2). In identity theory terms: this brain activation IS the conscious experience, or at least part of the brain activation that IS the conscious experience (note that the conceptual distinctions made apply in varying degrees whether identity theory, epiphenomenalism, or even dualism is adopted as the metaphysical starting point). Many NCC studies find certain neural events to correlate with conscious experience, and leave it at that. This is unfortunate, since a deeper reflection on what a given NCC finding can mean, the role it can play in the greater mechanisms underlying consciousness of emergent or downward causation (Feinberg, 2012; Hohwy, 2009; Neisser, 2012) might improve our understanding of the literature and better inform follow-up work.

\section{The case of binocular rivalry}

Binocular rivalry (see Part I) is an excellent example for our framework, considering the wide range of NCCs that have resulted from studies on binocular rivalry. Visual consciousness during binocular rivalry has been correlated with activity at some of the earliest stages of visual processing, from the LGN (Haynes, Deichmann, \& Rees, 2005; Wunderlich, Schneider, \& Kastner, 2005) and V1 (Haynes \& Rees, 2005; Lee, Blake, \& Heeger, 2005; Polonsky, Blake, Braun, \& Heeger, 2000; Tong \& Engel, 2001) all the way up the hierarchy through extrastriate regions (Lumer, Friston, \& Rees, 1998; Moutoussis, Keliris, Kourtzi, \& Logothetis, 2005; Tong, Nakayama, Vaughan, \& Kanwisher, 1998), parietal cortex (Lumer, Friston, \& Rees, 1998), and finally, even frontal regions (Lumer, Friston, \& Rees, 1998; Panagiotaropoulos, Deco, Kapoor, \& Logothetis, 2012). All of these regions are thus NCCs of visual consciousness during rivalry, but it seems unlikely to us and others (Miller, 2001, 2007, this volume; Crick and Koch, 1998; Logothetis, 1998, Revonsuo, 2000, 2001) that they all play the same role with respect to consciousness. Because of this widespread distribution, it is entirely possible to envision many different scenarios within the constraints of our prerequisitessubstrates-consequences framework. As we discuss in this chapter, V1 has been discounted by some as a candidate region for the actual substrates of consciousness. Thus, perhaps NCCs early in the hierarchy, such as LGN and V1, are prerequisites for conscious experience, while parietal and extrastriate regions are substrates of the experience, and activity in frontal regions reflects various consequences. But, without 
further evidence, the division of roles could just as easily be completely different. How to proceed in obtaining such extra evidence is what we discuss below. ${ }^{3}$

\section{Strategies to disentangle the entanglement}

We would like to know of all the NCCs that we are seeing in a particular experiment which is which: for each activated brain area, modulated frequency spectrum, ERP peak, are we looking at a substrate, a prerequisite, or a consequence? Is this possible? We propose five strategies for disentangling this entanglement.

\section{Cross-literature integration}

Content-invariant (or modality-specific) neural prerequisites or consequences of consciousness might be retrievable from the literature. We and others before us (e.g. (Dehaene and Naccache, 2001; Rees, 2007) have noted, for example, that frontoparietal activations seem to pop up in a wide range of consciousness paradigms. For example in multistable paradigms (Lumer, Friston et al., 1998; Lumer and Rees, 1999; Schoth, Waberski et al., 2007; Sterzer, Kleinschmidt et al., 2009) and ON-OFF paradigms (Dehaene, Naccache et al., 2001; Dehaene, Naccache et al., 1998). This suggests that frontoparietal activity is not content-specific, and therefore not (by itself) a neural substrate of any specific conscious experience, but perhaps a prerequisite or

\footnotetext{
${ }^{3}$ Recent related work has appeared. Aside from aforementioned Neisser (2012), Hohwy (2009) has pointed out a methodological/interpretational issue with the contrastive (content-consciousness) method: obtained NCCs might not reflect the phenomenality of our conscious experience, but rather the selection of one conscious experience over another (see also Miller 2001, 2007 on the problem of distinguishing attentional selection and visual consciousness during rivalry, and see further discussion below). Indeed, for any given NCC, this seems possible. Hidden in the totality of observable brain processes during (and immediately preceding) a conscious experience, must be both the phenomenality (corresponding to our 'substrate') and the selection (corresponding to our 'prerequisites'). And indeed, it is inherent to our schema that whenever a conscious experience X occurs, the preconditions P and consequences Q, always co-occur. Miller (2001, 2007 ) is not very optimistic about this problem. As mentioned above, he makes a distinction between two NCCs: the neural correlates and the neural constitution (or constituents) of consciousness. It is unclear in any given NCC paradigm, whether the results (the correlates) are constituents or not. The constituents are the neural processes/events/etcetera, that really are the conscious experience. They seemingly overlap perfectly with what we call substrates. Miller goes to some length explaining why we may never be able to fully parse these constituents from the correlates. It may be, he argues, a fundamental problem, a hard problem, with as yet no foreseeable solution. However, as mentioned above, Miller $(2001,2007)$ does agree with us (de Graaf et al., 2012) and others (Aru et al., 2012) that significant progress can nevertheless be made using appropriate strategies.
} 
consequence. Concretely, frontal and/or parietal cortex activation might reflect the attentional selection of 'consciousness-candidate'-items and thus be a prerequisite, or might activate because the consciously perceived item captures attention or primes a response and thus be a consequence (see Chapter III.1).

To search further in the NCC literature for activations or processes that reiterate across NCC studies, stimuli, and paradigms, newly emerging brain activation databases might be used. For instance, BrainMap holds a large number of published PET and fMRI studies, enabling meta-analyses and cross-study overviews (Fox and Lancaster, 2002). Related to this, for each candidate NCC, we must take into account basic anatomical knowledge converging with previous research. Since frontoparietal areas seem to play some important role in the establishment of consciousness, it stands to reason that NCCs serving as substrate would be connected to these (see Panagiotaropoulos et al., 2012). V1 is not connected in this way, which could be a reason to doubt V1 as a neural substrate, making it more likely as a prerequisite (Crick and Koch, 1995).

\section{Brain interference methods}

If we have determined that an NCC is unlikely to be a substrate of consciousness, and we hypothesize that it might be a prerequisite or consequence, we need to move forward. How can we distinguish between neural prerequisites and consequences? Again using frontoparietal cortices as an example, one could hypothesize that frontal or parietal regions drive the selection of one or other item for consciousness (e.g. by initiating a re-evaluation of the input; Sterzer et al., 2009; see also Carmel, Walsh et al., 2010; Kanai, Bahrami et al., 2010; Zaretskaya, Thielscher et al., 2010). On the other hand, perhaps frontal and/or parietal cortices are activated as a consequence of a salient new conscious experience (e.g. de Graaf, de Jong et al., 2011: Chapters III.1, III.2). When the NCC is a regional activation (as opposed to for example a chemical concentration level or interregional coherence), there is now an experimental method that may distinguish between regions that are functionally relevant (necessary) for a task or percept, and regions that are not. This is brain interference.

Transcranial magnetic stimulation (TMS) or transcranial direct current stimulation (tDCS) allow us to change brain activity in relatively constrained brain regions in healthy, awake, conscious human beings. Thus, the level of activity (more precisely: cortical excitability) in a region becomes an independent variable (Sack, Hubl et al., 2002). If frontal cortex activation of a certain level is a neural prerequisite for consciousness, TMS over frontal regions should interfere with conscious perception. TMS over early visual regions has repeatedly shown that it has the ability in principle to affect conscious (and unconscious) perception (Amassian, Cracco et al., 1989; Amassian, Cracco et al., 1993; Boyer, Harrison et al., 2005; de Graaf, Cornelsen et al., 2011; de Graaf, Goebel et al., 2012; de Graaf, Herring et al., 2011; Koivisto, Mantyla et al., 2010; Koivisto, Railo et al., 2011; Ro, Shelton et al., 2004; Silvanto, Lavie et al., 2005; Jacobs, Goebel et al., 2011), and it has recently been applied to frontal (de Graaf, de Jong et al., 2011) and parietal (Carmel, Walsh et al., 2010; de Graaf, de Jong et al., 
2011; Kanai, Bahrami et al., 2010; Miller et al., 2000; Zaretskaya, Thielscher et al., 2010) regions to start evaluating the frontoparietal role in multistable perception and other paradigms (e.g. Amassian, Mari et al., 2008).

It is clear that there are many possible instantiations of how the brain establishes conscious vision. As Hohwy and Neisser recently pointed out, there is likely a complex cascade of causal relations underlying any conscious experience, and the contrastive analysis only yields one piece of the puzzle (and not necessarily always the one we think). For example, maybe an extrastriate (e.g. face) area needs to connect with a frontal region in order to make a percept globally available and thus conscious. The connectivity itself might be the neural substrate of consciousness, rather than the level of activity in some region or other (Massimini, Ferrarelli et al., 2005; Tononi, 2004, 2005). This is certainly possible, and would complicate things. Yet, affecting the excitability of one region might also affect other anatomically/functionally connected areas (Bestmann, Baudewig et al., 2003, 2004; de Graaf, Jacobs et al., 2009; Ruff, Bestmann et al., 2008; Ruff, Blankenburg et al., 2006; Sack, Kohler et al., 2007), allowing it to potentially affect networks. Moreover, brain interference methods are becoming increasingly sophisticated, going beyond 'virtual lesions' (Pascual-Leone, Walsh et al., 2000) for example to induce frequency-specific oscillations in brain regions (Romei, Gross et al., 2010; Thut, Schyns et al., 2011; Thut, Veniero et al., 2011). This is very exciting in our view, since TMS might be used to test NCCs found in $\mathrm{EEG} / \mathrm{MEG}$ research.

Aru et al (2012) point out that lesion studies can serve a similar purpose. They draw on an interesting example involving neurons in the medial temporal lobe (MTL). These neurons fire in close correspondence to a subjective percept, thus constituting an NCC (Quiroga, Mukamel et al., 2008). Yet, lesions to this brain area do not eliminate conscious perception, so this NCC is not substrate and not prerequisite. The MTL NCC must be a neural consequence (discussed further below).

\section{Temporal information}

At the risk of pointing out the obvious: any NCC that occurs prior to a conscious experience cannot be a substrate, or consequence. In this sense, a division of NCCs in the temporal domain, related to the onset/period of a conscious experience, is useful. For instance, pre-stimulus alpha power determines whether a liminal stimulus will be perceived (van Dijk, Schoffelen et al., 2008), and even the phase of alpha-waves has a strong influence (Busch, Dubois et al., 2009; Mathewson, Gratton et al., 2009; see Chapter III.3). These NCCs must be neural prerequisites of conscious experience. For brain processes occurring after stimulus presentation (e.g. the $200 \mathrm{~ms}$ ERP negativity increase; (Koivisto, Lahteenmaki et al., 2008; Koivisto and Revonsuo, 2009), the question is more difficult. Such processes are not automatically neural consequences of conscious experience, since it is not straightforward at which point in time our phenomenal awareness of a stimulus 'happens' (Breitmeyer and Ogmen, 2006; Koch, 2004; Wilenius and Revonsuo, 2007). 
Melloni et al. (2011) recently showed how some neural prerequisites of consciousness, short-latency ERP components occurring early after stimulus presentation and previously thought to be 'NCC proper' (Pins and Ffytche, 2003), could be detected using experimental manipulations (Aru, Bachmann et al., 2012). Affecting the conscious experience first by attention, then by expectations, led to the same conscious percept but different 'background conditions'. Some components of the ERP were invariant to the manipulation (and thus remain NCC-substrate candidates), others were specific to either attention or expectation (thus unlikely to be NCC-substrates). Such examples cannot unequivocally determine NCC-substrates maybe (see below), but they certainly serve to refine our understanding and constrain our search for the NCCs.

Other methods are increasingly applied that operate in the temporal domain even if temporal resolution is notoriously low. In fMRI, there are BOLD chronometry (Formisano and Goebel, 2003) and connectivity methods (dynamic causal modeling; (Friston, Harrison et al., 2003); Granger causality mapping; (Goebel, Roebroeck et al., 2003; Roebroeck, Formisano et al., 2005). While perhaps not perfectly separable in time, neural consequences of consciousness should generally occur after neural substrates of consciousness. If a given network of brain regions in fMRI is an NCC, connectivity methods or BOLD chronometry might determine how information flows within this network: regions receiving information in later stages of this interactive framework are perhaps more likely to be neural consequences.

\section{Unconscious perception paradigms}

By thus tracking down neural prerequisites and neural consequences of consciousness, we can engage in a process of elimination. A network of regions found to correlate to consciousness can be evaluated node by node, using brain interference methods or other methods described in de Graaf et al. (2012) and Aru et al. (2012). Part of the strategy thusfar proposed is checking which NCCs are content-specific. Neural substrates are likely to be content-specific, but can we say that a content-specific neural event is automatically a substrate? Unfortunately not; we have already discussed contentspecific prerequisites/consequences. Nevertheless, we might make advances in this respect by looking for "neural correlates of unconsciousness". Motion area hMT/V5 for example is a content-specific part of an NCC of any conscious motion perception. Some might suggest that the conscious perception of motion thus resides in this motion area (Zeki, 2003, 2008). A key approach then, is to check to what extent such a region is active when motion is presented but not perceived consciously. Thus, unconscious perception paradigms can be used.

Recent studies using various stimuli and paradigms have shown that several modular brain regions in the visual hierarchy can display activity without conscious perception (Dehaene, Naccache et al., 2001; Fang and He, 2005; Haynes, Driver et al., 2005; Luck, Vogel et al., 1996; Marois, Yi et al., 2004; Morris, Ohman et al., 1999; Moutoussis and Zeki, 2002, 2006; Naccache, Gaillard et al., 2005; Pasley, Mayes et al., 2004; Rees, Wojciulik et al., 2000, 2002; Vuilleumier and Driver, 2007; Jacobs and Sack, 2012). For example, studies showing that the face processing area FFA is active 
when faces are presented but not perceived (e.g. Moutoussis and Zeki, 2002) suggest that some activity in the face area is not sufficient for a conscious face percept. The visual word form area may be active when words are presented (Dehaene, Naccache et al., 2001) but this does not mean that these words make it to consciousness. One might object that the activity level is lower in these studies. But Goebel et al. (2001) showed in a blindsight patient that activity in motion area hMT/V5 was of the same level when a motion stimulus was consciously perceived (when presented in the intact hemifield), versus when it was not at all perceived (when presented in the scotoma). Thus, even a 'normal' amount of activity in such a modular region seems insufficient to generate a conscious percept of motion.

Unfortunately, this strategy works only in one direction: null results (NOT replicating an NCC candidate during 'unconscious perception') seem difficult to interpret (see de Graaf and Sack, 2011; Chapter III.2) concerning null result interpretability). But, lessons from unconscious perception paradigms can surely eliminate content-specific candidates, and in our view have already done so for modular visual regions including at the very least hMT/V5 (see above). The next step seems to be to take into account combinations of different NCCs: is it for example sufficient to have simultaneous hMT/V5 and frontal activity? Or need there be connectivity between the two? Such questions might now be addressed in unconscious perception paradigms.

\section{Experimental manipulations to partition NCCs}

Above, we saw how Melloni et al. (2011) independently manipulated attention and expectation to tease apart NCC substrates from prerequisites/consequences. This relates to a general question whether attention might be confounding the NCC search. Among others, Koch and Tsuchiya $(2007,2012)$ believe that attention and consciousness are fundamentally different things. Yet, in NCC research, regions known to be involved in attention (i.e. frontoparietal regions: Corbetta, 1998; Coull, Frith et al., 1996; Naghavi and Nyberg, 2005; Nobre, Coull et al., 1999; Pessoa, Kastner et al., 2003) often pop up as correlating to consciousness, for example in multistable perception paradigms (Inui, Tanaka et al., 2000; Kleinschmidt, Buchel et al., 1998; Lumer, Friston et al., 1998; Schoth, Waberski et al., 2007; Sterzer, Russ et al., 2002). Thus, a debate rages on whether consciousness is nothing more than attention or attentional selection (e.g. Beck and Eccles, 1992; Koch and Tsuchiya, 2007; Mack and Rock, 1998; Merikle and Joordens, 1997; O'Regan and Noe, 2001; Posner, 1994; Velmans, 1996; see also Miller 2001; 2007).

Clearly, attention plays a role in the establishment of consciousness. Classic phenomena, such as the attentional blink (Luck, Vogel et al., 1996) and inattentional blindness (Mack and Rock, 1998) show that a lapse or (mis)allocation of attention can prevent a particular stimulus from reaching consciousness. But that doesn't mean attention and consciousness are the same thing. Attention could be an unavoidable prerequisite or consequence of conscious experience, or not even, if it just nearly always comingles with consciousness but is not per se an inevitable co-correlate. Partly exemplified by Melloni et al. (2011), attention could be manipulated independently 
from conscious experience. Orthogonal manipulations of conscious content and attention allocation could possibly tease apart the neural events underlying either/both. In fact, new light was shed on the role of V1 in consciousness, when a recent study employed a $2 \times 2$ design to orthogonally manipulate stimulus visibility and attention, as already predicted by (Koch and Tsuchiya, 2007, 2012): V1 was responsive to attentional changes, but not at all to conscious perception changes (Watanabe, Cheng et al., 2011).

A second confounder that might affect a wide range of NCC studies is task performance. Behavioral performance required by NCC paradigms (some behavioral marker of the conscious experience is necessary in almost all NCC studies) is one form of neural consequence of conscious experience. After all, as Lau (2008) points out; when consciousness differs, performance often differs as well. Ingeniously, Lau and Passingham (2006) used a metacontrast masking paradigm that contained conditions with equal task performance but unequal conscious perception. This was possible because the metacontrast function is a u-curve, and the u-curves for conscious (subjective) perception of stimuli and for correct visual discrimination performance were not in perfect overlap in their study. Having thus controlled for behavioral task performance, Lau and Passingham (2006) noted that only dorsolateral prefrontal cortex showed activity covarying with the conscious percept. Thus, while generally masking paradigms elicited a frontoparietal network, this finding suggests that some of the parietal activations might not have been NCC substrates.

It is promising then, that experimental manipulations, going a little further than classical contrastive methods are able to 'zoom in' on the NCC-substrates, eliminating neural prerequisites and consequences from the candidates thusfar obtained.

\section{Can we thus find the NCC substrates?}

Miller (2007) claims that the neural constituents, as opposed to correlates, of consciousness may not be found unequivocally by neuroscience. Not only now, but by any imaginable methods. He addresses several of the strategies that we've proposed, even enhances their detection power and spatiotemporal resolution for the sake of argument, and then argues why they would still not solve the problem. For example, he imagines a super-version of TMS, in which particular neurons or neuronal circuits can be stimulated, perhaps even disrupted perfectly. Imagine that such a method would fully be able to shut down at will some NCC candidate. Imagine then that the conscious percept would be absent. Could we conclude that the NCC is the substrate? No, perhaps it is a prerequisite. Imagine then that the conscious percept would be unaffected. Could we conclude that the NCC is a neural consequence? No. To see why, we must go back to the example of the medial temporal lobe (MTL), discussed above. Damage to MTL did not affect conscious perception, so we were happy to conclude that this particular NCC was a consequence. But the brain is known to be redundant, able to compensate for disrupted processing in some regions by moving task processing to other regions (e.g. Sack, Camprodon et al., 2005). Thus, it is possible that in healthy subjects, the MTL neurons ARE a prerequisite, or even a substrate, but that the brain establishes 
conscious perception in a different way, in different neurons, if the MTL is out of commission. There are two ways to address this: 1) if the MTL is, based on previous knowledge, plausibly involved in another processing task, such as entering items into memory systems when/after they are consciously perceived, this serves as converging evidence for a NCC-consequence. While Miller (2007) may be right that we can never definitively know, we make an educated guess, which for empiricists might be good enough. 2) the compensation hypothesis can be tested, using reversible virtual lesions (brain interference methods) concurrently with brain imaging. This can further strengthen the case, if no compensating brain events are found when MTL is disrupted, although again the answer would not be definitive (Miller 2007).

In short, there is no conceptual way to argue against these arguments, and even against strategies we outlined above that were not directly addressed by Miller (2007), such objections could likely be made. So, we can never be sure. However, even conceding the issue fully, we believe it may become practically irrelevant. This does not mean it should not be considered, accepted, and taken seriously. But at the same time, it should not detract us from following exactly the strategies we proposed. We may never be entirely sure that the NCC substrate candidate we are left with, after eliminating various candidates that turned out to be (more plausibly) prerequisites or consequences, is actually constitutive. But we will understand a lot better the sequence of neural events underlying consciousness. Converging evidence from multiple strategies will paint a picture that will suggest a cascade of brain events, perhaps in frameworks such as proposed by Neisser (2012), in which some brain events are prerequisites, some substrates, and some consequences. It will be a more refined theoretical model than we have now. We therefore believe, perhaps overly optimistically, that Miller hits the nail on the head when he says: “... Future scientific work may shed enough light on the problem that any remaining uncertainties ... will appear trivial rather than substantial." Whether the NCC program will turn out like this or not, we will not know until we try.

\section{Conclusion}

At the very least, a conceptual understanding that NCCs can have three 'roles' in the cascade of brain events that surround a conscious percept is useful. Using new methods and analysis tools, armed with this new understanding we should be able to move forward in a science of consciousness to start disentangling the neural correlates of consciousness into prerequisites, consequences and candidate substrates. As we progress, reframing the findings in different kinds of causal schemes (Neisser, 2012) may partly address the inherent shortcomings of the contrastive analysis (Hohwy, 2009). While very different, all the contributions made to this topic seem to agree on this much: NCC research is valuable, interesting, and ready to take some steps forward. Even if we may never with certainty distinguish the three NCCs, there is quite some ground to be covered before we reach our epistemological limits. 


\section{References}

Amassian V, Mari Z, Sagliocco L, Hassan N, Maccabee P, Cracco JB, Cracco RQ, and Bodis-Wollner I. (2008). Perception of phosphenes and flashed alphabetical characters is enhanced by single-pulse transcranial magnetic stimulation of anterior frontal lobe: The thalamic gate hypothesis. Perception, 37: 375-88.

Amassian VE, Cracco RQ, Maccabee PJ, Cracco JB, Rudell A, and Eberle L. (1989). Suppression of visual perception by magnetic coil stimulation of human occipital cortex. Electroencephalogr Clin Neurophysiol, 74: 458-62.

Amassian VE, Cracco RQ, Maccabee PJ, Cracco JB, Rudell AP, and Eberle L. (1993). Unmasking human visual perception with the magnetic coil and its relationship to hemispheric asymmetry. Brain Res, 605: 312-6.

Aru J, Bachmann T, Singer W, and Melloni L. (2012). Distilling the neural correlates of consciousness. Neurosci Biobehav Rev, 36: 737-46.

Baars BJ. (1989). A cognitive theory of consciousness. New York: Cambridge University Press.

Bachmann T. (2009). Finding erp-signatures of target awareness: Puzzle persists because of experimental co-variation of the objective and subjective variables. Conscious Cogn, 18: 804-8; discussion 809-10.

Beck F and Eccles JC. (1992). Quantum aspects of brain activity and the role of consciousness. Proc Natl Acad Sci U S A, 89: 11357-61.

Bestmann S, Baudewig J, Siebner HR, Rothwell JC, and Frahm J. (2003). Subthreshold high-frequency tms of human primary motor cortex modulates interconnected frontal motor areas as detected by interleaved fmri-tms. Neuroimage, 20: 1685-96.

Bestmann S, Baudewig J, Siebner HR, Rothwell JC, and Frahm J. (2004). Functional mri of the immediate impact of transcranial magnetic stimulation on cortical and subcortical motor circuits. Eur J Neurosci, 19: 1950-62.

Blake R. (2001). A primer on binocular rivalry, including current controversies. Brain and Mind, 2: 5-38.

Blanke O. (2012). Multisensory brain mechanisms of bodily self-consciousness. Nat Rev Neurosci, 13: 556-71.

Block N. (2005). Two neural correlates of consciousness. Trends Cogn Sci, 9: 46-52.

Boyer JL, Harrison S, and Ro T. (2005). Unconscious processing of orientation and color without primary visual cortex. Proc Natl Acad Sci U S A, 102: 16875-9.

Breese BB. (1899). On inhibition. Psychological Monographs, 3: 1-65.

Breitmeyer B and Ogmen H. (2006). Visual masking: Time slices through conscious and unconscious vision. New York: Oxford University press.

Busch NA, Dubois J, and VanRullen R. (2009). The phase of ongoing eeg oscillations predicts visual perception. J Neurosci, 29: 7869-76.

Carmel D, Walsh V, Lavie N, and Rees G. (2010). Right parietal tms shortens dominance durations in binocular rivalry. Curr Biol, 20: R799-800.

Chalmers D. (2000). What is a neural correlate of consciousness? In T. M. (Ed.) Neural correlates of consciousness: Empirical and conceptual questions: MIT Press.

Chalmers DJ. (1996). The conscious mind: In search of a fundamental theory. Oxford: Oxford University Press. 
Corbetta M. (1998). Frontoparietal cortical networks for directing attention and the eye to visual locations: Identical, independent, or overlapping neural systems? Proc Natl Acad Sci U S A, 95: 831-8.

Coull JT, Frith CD, Frackowiak RS, and Grasby PM. (1996). A fronto-parietal network for rapid visual information processing: A pet study of sustained attention and working memory. Neuropsychologia, 34: 1085-95.

Crick F. (1994). The astonishing hypothesis: The scientific search for the soul. New York: Touchstone.

Crick F and Koch C. (1995). Are we aware of neural activity in primary visual cortex? Nature, 375: 121-3.

Crick F and Koch C. (1998). Consciousness and neuroscience. Cereb Cortex, 8: 97-107. de Graaf TA, Cornelsen S, Jacobs C, and Sack AT. (2011). Tms effects on subjective and objective measures of vision: Stimulation intensity and pre- versus post-stimulus masking. Conscious Cogn, 20: 1244-55.

de Graaf TA, de Jong MC, Goebel R, van Ee R, and Sack AT. (2011). On the functional relevance of frontal cortex for passive and voluntarily controlled bistable vision. Cereb Cortex, 21: 2322-31.

de Graaf TA, Goebel R, and Sack AT. (2012). Feedforward and quick recurrent processes in early visual cortex revealed by tms? Neuroimage.

de Graaf TA, Herring J, and Sack AT. (2011). A chronometric exploration of highresolution 'sensitive tms masking' effects on subjective and objective measures of vision. Exp Brain Res, 209: 19-27.

de Graaf TA, Hsieh PJ, and Sack AT. (2012). The 'correlates' in neural correlates of consciousness. Neurosci Biobehav Rev, 36: 191-7.

de Graaf TA, Jacobs C, Roebroeck A, and Sack AT. (2009). Fmri effective connectivity and tms chronometry: Complementary accounts of causality in the visuospatial judgment network. PLoS One, 4: e8307.

de Graaf TA and Sack AT. (2012). Null results in tms: From absence of evidence to evidence of absence. Neurosci Biobehav Rev, 35: 871-7.

Dehaene S and Naccache L. (2001). Towards a cognitive neuroscience of consciousness: Basic evidence and a workspace framework. Cognition, 79: 1-37.

Dehaene S, Naccache L, Cohen L, Bihan DL, Mangin JF, Poline JB, and Riviere D. (2001). Cerebral mechanisms of word masking and unconscious repetition priming. Nat Neurosci, 4: 752-8.

Dehaene S, Naccache L, Le Clec HG, Koechlin E, Mueller M, Dehaene-Lambertz G, van de Moortele PF, and Le Bihan D. (1998). Imaging unconscious semantic priming. Nature, 395: 597-600.

Dierks T, Linden DE, Jandl M, Formisano E, Goebel R, Lanfermann H, and Singer W. (1999). Activation of heschl's gyrus during auditory hallucinations. Neuron, 22: 615-21. Edelman GM and Tononi G. (2000). Universe of consciousness: How matter becomes imagination. New York: Basic Books.

Fang F and He S. (2005). Cortical responses to invisible objects in the human dorsal and ventral pathways. Nat Neurosci, 8: 1380-5.

Feinberg TE. (2012). Neuroontology, neurobiological naturalism, and consciousness: A challenge to scientific reduction and a solution. Phys Life Rev, 9: 13-34. 
Ferrarelli F, Massimini M, Sarasso S, Casali A, Riedner BA, Angelini G, Tononi G, and Pearce RA. (2010). Breakdown in cortical effective connectivity during midazolaminduced loss of consciousness. Proc Natl Acad Sci U S A, 107: 2681-6.

Formisano E and Goebel R. (2003). Tracking cognitive processes with functional mri mental chronometry. Curr Opin Neurobiol, 13: 174-81.

Fox PT and Lancaster JL. (2002). Opinion: Mapping context and content: The brainmap model. Nat Rev Neurosci, 3: 319-21.

Fox R. (1991). Binocular rivalry. In Regan D.M. (Ed.) Binocular vision and psychophysics. London: MacMillan Press, pp. 93-110.

Friston KJ, Harrison L, and Penny W. (2003). Dynamic causal modelling. Neuroimage, 19: 1273-302.

Gallagher S and Zahavi D. (2010). Phenomenological approaches to self-consciousness. In Zalta E.N. (Ed.) The stanford encyclopedia of philosophy (winter 2010 edition): http://plato.stanford.edu/archives/win2010/entries/self-consciousness-

phenomenological/.

Goebel R, Muckli L, Zanella FE, Singer W, and Stoerig P. (2001). Sustained extrastriate cortical activation without visual awareness revealed by fmri studies of hemianopic patients. Vision Res, 41: 1459-74.

Goebel R, Roebroeck A, Kim DS, and Formisano E. (2003). Investigating directed cortical interactions in time-resolved fmri data using vector autoregressive modeling and granger causality mapping. Magn Reson Imaging, 21: 1251-61.

Gosseries O, Bruno MA, Chatelle C, Vanhaudenhuyse A, Schnakers C, Soddu A, and Laureys S. (2011). Disorders of consciousness: What's in a name? NeuroRehabilitation, 28: 3-14.

Gusnard DA. (2005). Being a self: Considerations from functional imaging. Conscious Cogn, 14: 679-97.

Haynes, J. D., Deichmann, R., \& Rees, G. (2005). Eye-specific effects of binocular rivalry in the human lateral geniculate nucleus. Nature, 438(7067), 496-499.

Haynes, J. D., \& Rees, G. (2005). Predicting the stream of consciousness from activity in human visual cortex. Curr Biol, 15(14), 1301-1307.

Haynes JD, Driver J, and Rees G (2005). Visibility reflects dynamic changes of effective connectivity between v1 and fusiform cortex. Neuron, 46: 811-21.

Hohwy J. (2009). The neural correlates of consciousness: New experimental approaches needed? Conscious Cogn, 18: 428-38.

Howard LP and Rogers BJ. (1995). Binocular vision and stereopsis. New York: Oxford University Press.

Inui T, Tanaka S, Okada T, Nishizawa S, Katayama M, and Konishi J. (2000). Neural substrates for depth perception of the necker cube; a functional magnetic resonance imaging study in human subjects. Neurosci Lett, 282: 145-8.

Jacobs C, Goebel R, and Sack AT. (2011).Visual awareness suppression by prestimulus brain stimulation; a neural effect. Neuroimage.

Jacobs C and Sack AT. (2012). Behavior in oblivion: The neurobiology of subliminal priming. Brain Sciences, 2: 225-241.

Kanai R, Bahrami B, and Rees G. (2010). Human parietal cortex structure predicts individual differences in perceptual rivalry. Curr Biol. 
Kim CY and Blake R. (2005). Psychophysical magic: Rendering the visible 'invisible'. Trends Cogn Sci, 9: 381-8.

Kleinschmidt A, Buchel C, Zeki S, and Frackowiak RS. (1998). Human brain activity during spontaneously reversing perception of ambiguous figures. Proc Biol Sci, 265: 2427-33.

Koch C. (2004). The quest for consciousness: A neurobiological approach. Englewood, US-CO: Roberts \& Company Publishers.

Koch C and Tsuchiya N. (2007). Attention and consciousness: Two distinct brain processes. Trends Cogn Sci, 11: 16-22.

Koch C and Tsuchiya N. (2012). Attention and consciousness: Related yet different. Trends Cogn Sci, 16: 103-5.

Koivisto M, Lahteenmaki M, Sorensen TA, Vangkilde S, Overgaard M, and Revonsuo A. (2008). The earliest electrophysiological correlate of visual awareness? Brain Cogn, 66: 91-103.

Koivisto M, Mantyla T, and Silvanto J. (2010). The role of early visual cortex (v1/v2) in conscious and unconscious visual perception. Neuroimage, 51: 828-34.

Koivisto M, Railo H, and Salminen-Vaparanta N. (2011). Transcranial magnetic stimulation of early visual cortex interferes with subjective visual awareness and objective forced-choice performance. Conscious Cogn, 20: 288-98.

Koivisto M and Revonsuo A. (2009). Event-related brain potential correlates of visual awareness. Neurosci Biobehav Rev.

Lau HC. (2008). A higher order bayesian decision theory of consciousness. Prog Brain Res, 168: 35-48.

Lau HC and Passingham RE. (2006). Relative blindsight in normal observers and the neural correlate of visual consciousness. Proc Natl Acad Sci U S A, 103: 18763-8.

Lee, S. H., Blake, R., \& Heeger, D. J. (2005). Traveling waves of activity in primary visual cortex during binocular rivalry. Nat Neurosci, 8(1), 22-23.

Levelt W. (1965). On binocular rivalry. Soesterberg, Netherlands: Institute for Perception RVOTNO.

Logothetis N. (1998). Object vision and visual awareness. Curr Opin Neurobiol, 8: 53644.

Logothetis NK, Leopold DA, and Sheinberg DL. (1996). What is rivalling during binocular rivalry? Nature, 380: 621-4.

Luck SJ, Vogel EK, and Shapiro KL (1996). Word meanings can be accessed but not reported during the attentional blink. Nature, 383: 616-8.

Lumer ED, Friston KJ, and Rees G (1998). Neural correlates of perceptual rivalry in the human brain. Science, 280: 1930-4.

Lumer ED and Rees G (1999). Covariation of activity in visual and prefrontal cortex associated with subjective visual perception. Proc Natl Acad Sci U S A, 96: 1669-73.

Mack A and Rock I (1998). Inattentional blindness. Cambridge, MA: MIT Press.

Marois R, Yi DJ, and Chun MM (2004). The neural fate of consciously perceived and missed events in the attentional blink. Neuron, 41: 465-72.

Massimini M, Ferrarelli F, Huber R, Esser SK, Singh H, and Tononi G (2005). Breakdown of cortical effective connectivity during sleep. Science, 309: 2228-32. 
Mathewson KE, Gratton G, Fabiani M, Beck DM, and Ro T (2009). To see or not to see: Prestimulus alpha phase predicts visual awareness. J Neurosci, 29: 2725-32.

Melloni L, Schwiedrzik CM, Muller N, Rodriguez E, and Singer W (2011). Expectations change the signatures and timing of electrophysiological correlates of perceptual awareness. J Neurosci, 31: 1386-96.

Melloni L and Singer W (2010). Distinct characteristics of conscious experience are met by large scale neuronal synchronization. In Perry E., Collerton D., LeBeau F.E.N., and Ashton H. (Eds.), New horizons in the neuroscience of consciousness. Amsterdam: John Benjamins, pp. 17-28.

Merikle PM and Joordens S (1997). Parallels between perception without attention and perception without awareness. Conscious Cogn, 6: 219-36.

Miller SM (2001). Binocular rivalry and the cerebral hemispheres: With a note on the correlation and constitution of visual consciousness. Brain and Mind, 2: 119-149.

Miller SM (2007). On the correlation/constitution distinction problem (and other hard problems) in the scientific study of consciousness. Acta Neuropsychiatrica, 19: 159176.

Morris JS, Ohman A, and Dolan RJ (1999). A subcortical pathway to the right amygdala mediating "Unseen" Fear. Proc Natl Acad Sci U S A, 96: 1680-5.

Moutoussis, K., Keliris, G., Kourtzi, Z., \& Logothetis, N. (2005). A binocular rivalry study of motion perception in the human brain. Vision Res, 45(17), 2231-2243.

Moutoussis K and Zeki S (2002). The relationship between cortical activation and perception investigated with invisible stimuli. Proc Natl Acad Sci U S A, 99: 9527-32.

Moutoussis K and Zeki S (2006). Seeing invisible motion: A human fmri study. Curr Biol, 16: 574-9.

Naccache L, Gaillard R, Adam C, Hasboun D, Clemenceau S, Baulac M, Dehaene S, and Cohen L (2005). A direct intracranial record of emotions evoked by subliminal words. Proc Natl Acad Sci U S A, 102: 7713-7.

Naghavi HR and Nyberg L (2005). Common fronto-parietal activity in attention, memory, and consciousness: Shared demands on integration? Conscious Cogn, 14: 390425 .

Neisser J (2012). Neural correlates of consciousness reconsidered. Conscious Cogn, 21: 681-90.

Nobre AC, Coull JT, Frith CD, and Mesulam MM (1999). Orbitofrontal cortex is activated during breaches of expectation in tasks of visual attention. Nat Neurosci, 2: $11-2$.

Noë A and Thompson E (2004). Are there neural correlates of consciousness? Journal of Consciousness Studies, 11: 3-28.

O'Regan JK and Noe A (2001). A sensorimotor account of vision and visual consciousness. Behav Brain Sci, 24: 939-73; discussion 973-1031.

Owen AM, Coleman MR, Boly M, Davis MH, Laureys S, and Pickard JD (2006). Detecting awareness in the vegetative state. Science, 313: 1402.

Panagiotaropoulos, T. I., Deco, G., Kapoor, V., \& Logothetis, N. K. (2012). Neuronal discharges and gamma oscillations explicitly reflect visual consciousness in the lateral prefrontal cortex. Neuron, 74(5), 924-935. 
Pascual-Leone A, Walsh V, and Rothwell J (2000). Transcranial magnetic stimulation in cognitive neuroscience--virtual lesion, chronometry, and functional connectivity. Curr Opin Neurobiol, 10: 232-7.

Pasley BN, Mayes LC, and Schultz RT (2004). Subcortical discrimination of unperceived objects during binocular rivalry. Neuron, 42: 163-72.

Pessoa L, Kastner S, and Ungerleider LG (2003). Neuroimaging studies of attention: From modulation of sensory processing to top-down control. J Neurosci, 23: 3990-8.

Pins D and Ffytche D (2003). The neural correlates of conscious vision. Cereb Cortex, 13: 461-74.

Polonsky, A., Blake, R., Braun, J., \& Heeger, D. J. (2000). Neuronal activity in human primary visual cortex correlates with perception during binocular rivalry. Nat Neurosci, 3(11), 1153-1159.

Posner MI (1994). Attention: The mechanisms of consciousness. Proc Natl Acad Sci U $S$ A, 91: 7398-403.

Quiroga RQ, Mukamel R, Isham EA, Malach R, and Fried I (2008). Human singleneuron responses at the threshold of conscious recognition. Proc Natl Acad Sci US A, 105: 3599-604.

Rees G (2007). Neural correlates of the contents of visual awareness in humans. Philos Trans R Soc Lond B Biol Sci, 362: 877-86.

Rees G, Wojciulik E, Clarke K, Husain M, Frith C, and Driver J (2000). Unconscious activation of visual cortex in the damaged right hemisphere of a parietal patient with extinction. Brain, 123 ( Pt 8): 1624-33.

Rees G, Wojciulik E, Clarke K, Husain M, Frith C, and Driver J (2002). Neural correlates of conscious and unconscious vision in parietal extinction. Neurocase, 8: 387-93.

Revonsuo A (2000). Prospects for a scientific research program on consciousness. In Metzinger T. (Ed.) Neural correlates of consciousness: Empirical and conceptual questions. Cambridge: MIT Press.

Revonsuo A (2001). Can functional brain imaging discover consciousness in the brain? Journal of Consciousness Studies, 8: 3-23.

Ro T, Shelton D, Lee OL, and Chang E (2004). Extrageniculate mediation of unconscious vision in transcranial magnetic stimulation-induced blindsight. Proc Natl Acad Sci U S A, 101: 9933-5.

Roebroeck A, Formisano E, and Goebel R (2005). Mapping directed influence over the brain using granger causality and fmri. Neuroimage, 25: 230-42.

Romei V, Gross J, and Thut G (2010). On the role of prestimulus alpha rhythms over occipito-parietal areas in visual input regulation: Correlation or causation? J Neurosci, 30: $8692-7$

Ruff CC, Bestmann S, Blankenburg F, Bjoertomt O, Josephs O, Weiskopf N, Deichmann R, and Driver J (2008). Distinct causal influences of parietal versus frontal areas on human visual cortex: Evidence from concurrent tms-fmri. Cereb Cortex, 18: 817-27.

Ruff CC, Blankenburg F, Bjoertomt O, Bestmann S, Freeman E, Haynes JD, Rees G, Josephs O, Deichmann R, and Driver J (2006). Concurrent tms-fmri and psychophysics reveal frontal influences on human retinotopic visual cortex. Curr Biol, 16: 1479-88. 
Sack AT, Camprodon JA, Pascual-Leone A, and Goebel R (2005). The dynamics of interhemispheric compensatory processes in mental imagery. Science, 308: 702-4.

Sack AT, Hubl D, Prvulovic D, Formisano E, Jandl M, Zanella FE, Maurer K, Goebel R, Dierks T, and Linden DE (2002). The experimental combination of rtms and fmri reveals the functional relevance of parietal cortex for visuospatial functions. Brain Res Cogn Brain Res, 13: 85-93.

Sack AT, Kohler A, Bestmann S, Linden DE, Dechent P, Goebel R, and Baudewig J (2007). Imaging the brain activity changes underlying impaired visuospatial judgments: Simultaneous fmri, tms, and behavioral studies. Cereb Cortex, 17: 2841-52.

Schoth F, Waberski TD, Krings T, Gobbele R, and Buchner H (2007). Cerebral processing of spontaneous reversals of the rotating necker cube. Neuroreport, 18: 13358 .

Seth AK (2009). Functions of consciousness. In Banks W.P. (Ed.) Encyclopedia of consciousness. Amsterdam: Elsevier, p. 279/293.

Silva S, Alacoque X, Fourcade O, Samii K, Marque P, Woods R, Mazziotta J, Chollet F, and Loubinoux I (2010). Wakefulness and loss of awareness: Brain and brainstem interaction in the vegetative state. Neurology, 74: 313-20.

Silvanto J (2008). A re-evaluation of blindsight and the role of striate cortex (v1) in visual awareness. Neuropsychologia, 46: 2869-71.

Silvanto J, Lavie N, and Walsh V (2005). Double dissociation of v1 and v5/mt activity in visual awareness. Cereb Cortex, 15: 1736-41.

Sterzer P, Kleinschmidt A, and Rees G (2009). The neural bases of multistable perception. Trends Cogn Sci, 13: 310-8.

Sterzer P, Russ MO, Preibisch C, and Kleinschmidt A (2002). Neural correlates of spontaneous direction reversals in ambiguous apparent visual motion. Neuroimage, 15: 908-16.

Thut G, Schyns PG, and Gross J (2011). Entrainment of perceptually relevant brain oscillations by non-invasive rhythmic stimulation of the human brain. Front Psychol, 2: 170 .

Thut G, Veniero D, Romei V, Miniussi C, Schyns P, and Gross J (2011). Rhythmic tms causes local entrainment of natural oscillatory signatures. Curr Biol, 21: 1176-85.

Tong F (2003). Primary visual cortex and visual awareness. Nat Rev Neurosci, 4: 219 29.

Tong F, Meng M, and Blake R (2006). Neural bases of binocular rivalry. Trends Cogn Sci, 10: 502-11.

Tong F, Nakayama K, Vaughan JT, and Kanwisher N (1998). Binocular rivalry and visual awareness in human extrastriate cortex. Neuron, 21: 753-9.

Tong, F., \& Engel, S. A. (2001). Interocular rivalry revealed in the human cortical blind-spot representation. Nature, 411(6834), 195-199.

Tononi G (2004). An information integration theory of consciousness. BMC Neurosci, 5: 42.

Tononi G (2005). Consciousness, information integration, and the brain. Prog Brain Res, 150: 109-26. 
van Dijk H, Schoffelen JM, Oostenveld R, and Jensen O (2008). Prestimulus oscillatory activity in the alpha band predicts visual discrimination ability. J Neurosci, 28: 181623.

Velmans M (1996) (Ed.). The science of consciousness. London: Routledge.

von der Heydt R, Peterhans E, and Baumgartner G (1984). Illusory contours and cortical neuron responses. Science, 224: 1260-62.

Vuilleumier P and Driver J (2007). Modulation of visual processing by attention and emotion: Windows on causal interactions between human brain regions. Philos Trans $R$ Soc Lond B Biol Sci, 362: 837-55.

Watanabe M, Cheng K, Murayama Y, Ueno K, Asamizuya T, Tanaka K, and Logothetis N (2011). Attention but not awareness modulates the bold signal in the human v1 during binocular suppression. Science, 334: 829-31.

Weil RS and Rees G (2011). A new taxonomy for perceptual filling-in. Brain Research Reviews, 67: 40-55.

Weiskrantz L (1996). Blindsight revisited. Curr Opin Neurobiol, 6: 215-20.

Wheatstone C (1838). On some remarkable, and hitherto unobserved, phenomena of binocular vision. Phil. Trans. R. Soc. Lond., 128: 371-394.

Wilenius ME and Revonsuo AT (2007). Timing of the earliest erp correlate of visual awareness. Psychophysiology, 44: 703-10.

Wunderlich, K., Schneider, K. A., \& Kastner, S. (2005). Neural correlates of binocular rivalry in the human lateral geniculate nucleus. Nat Neurosci, 8(11), 1595-1602.

Zaidi Q, Ennis R, Cao D, and Lee B (2012). Neural locus of color afterimages. Curr Biol, 22: 220-4.

Zaretskaya N, Thielscher A, Logothetis NK, and Bartels A (2010). Disrupting parietal function prolongs dominance durations in binocular rivalry. Curr Biol, 20: 2106-11.

Zeki S (2003). The disunity of consciousness. Trends Cogn Sci, 7: 214-218.

Zeki S (2008). The disunity of consciousness. Prog Brain Res, 168: 11-8. 
Part I

Low-level Aspects of Vision:

The Disrupted Rivalry Effect 



\section{The Disrupted Rivalry Effect}

Related publication(s):

De Graaf, TA, van Ee, R*, Croonenberg, D, Goebel, R, Sack, AT (in preparation). The disrupted rivalry effect: a new binocular rivalry phenomenon?.

* Raymond van Ee

1) Department of Physics of Man, Helmholtz Institute, Utrecht University, Utrecht 3584 $\mathrm{CH}$, The Netherlands

2) Department of Brain, Body, Behaviour, Philips Research Laboratories, Eindhoven 5656 AE, The Netherlands

3) Department of Experimental Psychology, Leuven University, Leuven B-3000, Leuven, Belgium 


\begin{abstract}
In binocular rivalry, two images presented separately to the two eyes compete for access to visual awareness. Conscious perception switches between both percepts, at a rate that depends on perceptual and cognitive state, and on stimulus parameters. In flash suppression, a binocular rivalry phenomenon, a newly presented image to one eye wins dominance over a previously adapted stimulus to the other eye. Here, we show that a potentially new phenomenon can occur if flash suppression is followed by quick removal of the newly presented stimulus. Thus, shortly after instigating binocular rivalry, rivalry is disrupted. Rather than conscious perception reverting back to the remaining salient stimulus, subjects report 'not seeing anything' for some time, before the remaining stimulus is indeed perceived again. To establish and probe this 'disrupted rivalry effect' (DRE), we performed a series of experiments using competing high-contrast peripheral 'star' and 'triangle' stimuli presented to the two eyes via a custom-built stereoscope. We show that the effect duration depends on the durations of the adaptation phase (time before flash suppression by second image) and rivalry phase (time between flash suppression and removal of the second image), that DRE is also present under fully focused attention, and that the duration varies across visual field rivalry locations. A simple 'double-inhibition model' seems suited to explain all the main findings, postulating DRE as a useful new phenomenon under the umbrella of binocular rivalry.
\end{abstract}




\section{General Introduction}

Binocular rivalry is an established and widely researched psychophysical paradigm, in which the two eyes are presented with two different images in a corresponding visual field location. In this paradigm, the conscious percept will vary stochastically, switching back and forth between both possible percepts (Levelt, 1965). Based on previous research, several models on the mechanisms behind binocular rivalry have been proposed (e.g. Blake, 1989; Freeman, Nguyen, \& Alais, 2005; Leopold \& Logothetis, 1996; Logothetis, Leopold, \& Sheinberg, 1996; Mueller \& Blake, 1989; Tong, Meng, \& Blake, 2006). One basic component of these models is reciprocal inhibition between the representations of both possible percepts. The fundamental idea is that when one representation wins the competition for dominance, it will gradually decrease in strength, while the currently suppressed representation recovers. After a period of time that depends on attentional state (Brascamp \& Blake, 2012) and various parameters affecting rivaling stimulus strength (Levelt, 1965), the suppressed representation will have recovered enough for the scales to tip, and the suppressed representation will become dominant again.

While the concept of reciprocal inhibition seems widely accepted, the details remain unclear. For example, different factors influence the durations of stimulus dominance and stimulus suppression (e.g. Sobel \& Blake, 2002). It has been proposed that competition takes place between neurons prior to binocular convergence (e.g. Lehky, 1988), monocular channels (e.g. Blake, 1989), neurons along the entire monocular pathway to higher-order cortex (e.g. Freeman, et al., 2005; Leopold \& Logothetis, 1996), and within higher-order cortex (e.g. Logothetis, et al., 1996). It is possible that all mechanisms take place simultaneously (Tong, et al., 2006) although perhaps their contributions may depend on stimulus and task parameters (e.g. Y. Bonneh, Sagi, \& Karni, 2001; Freeman, et al., 2005).

One of the competing representations can receive a substantial 'boost' in this mutual inhibition scheme, if it is presented after some delay. The basic form of this 'flash suppression' phenomenon involves the single presentation of one stimulus for some time (hundreds of milliseconds to seconds), before the second stimulus is presented to the other eye (Wolfe, 1984). Likely due to the fact that the previously dominant stimulus has already adapted to some extent, and possibly due to the visual system's propensity to value change in inputs whether this constitutes stimulus onset or offset (e.g. Macknik \& Livingstone, 1998; Roland, 2010), the second, 'fresh', stimulus will immediately gain dominance (but see Brascamp, Knapen, Kanai, van Ee, \& van den Berg, 2007).

The question is, however, what it would mean in this scenario if the flashed stimulus is removed almost immediately after presentation. On the one hand, intuitively one might expect that the remaining visual stimulus immediately becomes dominant again. After all, it is the only remaining stimulus, without any true competition. On the other hand, the second eye that was first presented with the flashed stimulus, which was subsequently removed again, has in quick succession received two changes in visual input: a high-contrast stimulus onset, and subsequent offset. Thus, in terms of recent visual change, the eye currently without any presented stimulus is much more 
'interesting' to the visual system. Recent work has indeed shown that visual transients can affect binocular rivalry (Kanai, Moradi, Shimojo, \& Verstraten, 2005), and make a salient monoptically presented stimulus disappear from conscious vision (Wilke, Logothetis, \& Leopold, 2003). How much of an influence can visual change have on binocular rivalry? Can it take precedence over salient visual stimulation?

We noticed in pilot experiments on binocular rivalry that disrupting rivalry seemed to have a strange effect. Concretely, when first adapting one eye to a salient high-contrast peripheral stimulus (adaptation phase), and then flash-suppressing this stimulus with a competing image to the other eye (rivalry phase) but immediately taking away again this image, 'nothing' (i.e. only homogeneous black background) was perceived for several seconds (disrupted rivalry phase). In the current study, we explored this 'disrupted rivalry effect' (DRE) to identify its sources, constraints, and parameters. Concretely, we aimed to characterize this effect by varying adaptation time, rivalry time, and keeping track of its duration in different visual field locations. We first explored the effect by presenting a circular array of eight simultaneously competing visual elements (i.e. stars and triangles) (experiment 1), using five levels of adaptation time and rivalry time, and recording which elements 'disappeared' for the longest time. We then presented only one visual element per trial to maximize the attention allocation to the relevant visual field location (experiment 2). We replicated the latter experiment while fixing trial length to better control for potential eye movements (experiment 3 ). Lastly, we increased the durations of the adaptation phase to tens of seconds to further probe its effect on the duration of DRE (experiment 4). Throughout, whenever possible we included a 'standard' binocular rivalry/flash suppression condition, and a control condition that closely mimicked the subjective-perceptual sequence but actually involved no rivalry, to compare and relate the disrupted rivalry effects to. 


\section{Experiment 1: the disrupted rivalry effect over the visual field}

\section{Introduction}

The first experiment aimed to formally establish the disrupted rivalry effect, and map its dependence on the duration of the initial 'adaptation phase', as well as the duration of the 'rivalry phase'. A simple mutual inhibition model of rivalry would predict an increased disrupted rivalry effect (DRE) with increased adaptation time, and decreased DRE with increased rivalry time (see General Discussion for details). Using an array of eight visual elements constituting a circle around a central fixation dot, all four visual field quadrants at once were stimulated and probed. Subjects indicated by button press after the disrupted rivalry phase when the first stimulus was again fully visible. Immediately after this, they indicated which of the eight visual elements was the last to re-appear. This later allowed us to evaluate the effect durations over visual field locations, to see if the effect was equally strong across the visual field or if its duration was inhomogeneous across the field.

\section{Material and Methods}

\section{Participants}

Twelve participants volunteered for this study, of which 10 were naive to the goals of the study and new to any binocular rivalry setup. All had normal or corrected-to-normal vision. They provided written informed consent in each of two experimental sessions. This and all subsequent experiments were approved by the local ethics committee.

\section{Stimuli}

Stimuli were custom-made bitmaps presented on two standard TFT monitors, using Presentation software (Neurobehavioral Systems, CA, USA). 'Blank' images contained a central fixation dot and a peripheral 'frame' to provide context for binocular fusion. 'Triangle' images moreover contained a circular array of eight triangle elements. The total circular array of elements was 9.5 visual degrees in diameter. Each element was 1.5 degrees in diameter, line thickness was 0.2 degrees. 'Star' images contained a circular array of star elements in the place of triangle elements, and were otherwise identical. See Figure 1 for the stimuli. The stimulus elements of triangles and stars were inspired from van Ee (2011). 


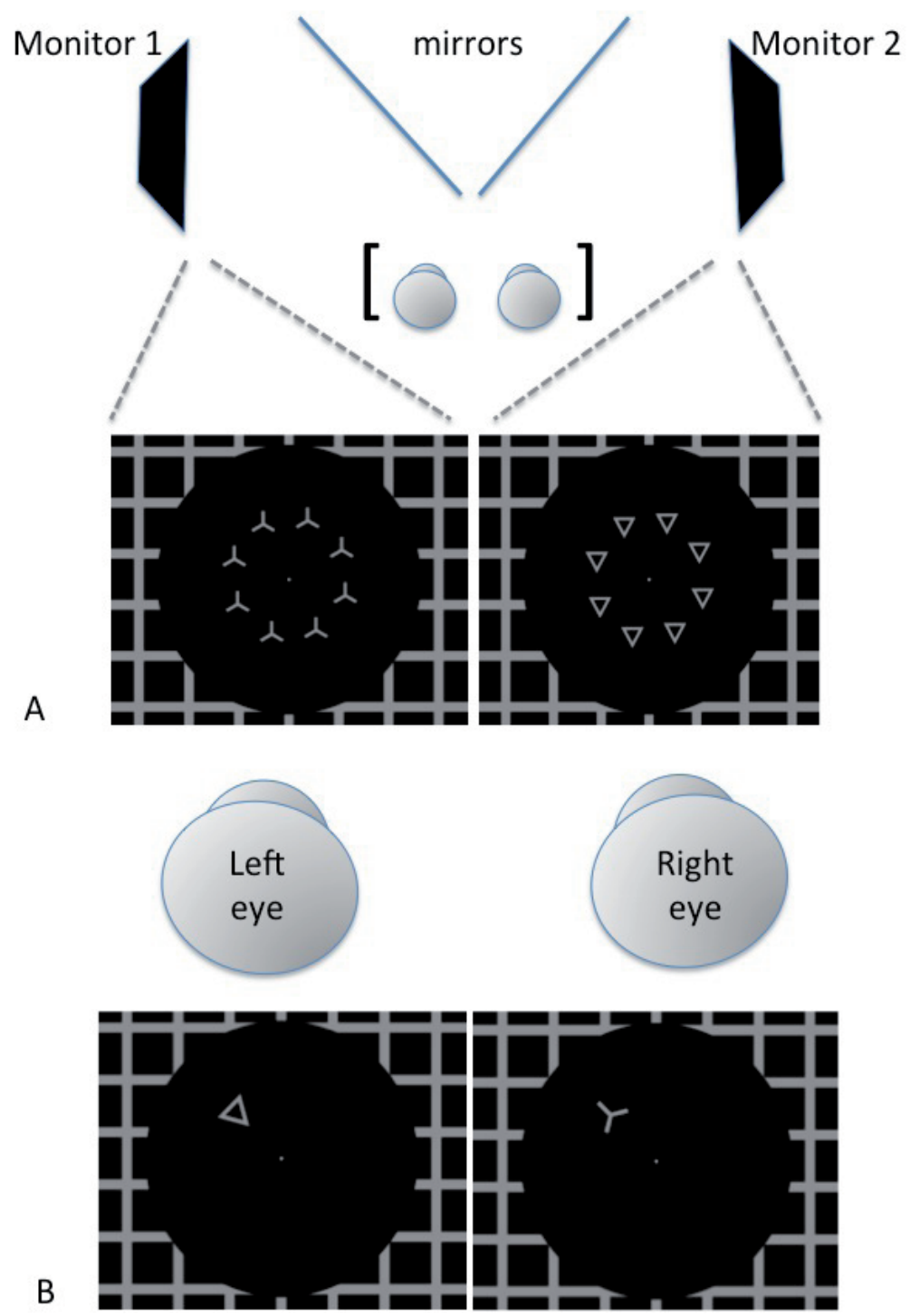

Figure 1: experimental setup and stimuli

A. We built a stereoscope containing two mirrors with adjustable positions and angles. To the right and left of these mirrors were two identical computer monitors (see Methods).

Participants were seated in a chin rest right in front of the mirrors, ensuring that the right and left eye could not perceive monitors' reflection in the left and right mirror respectively. They wore customized diving goggles without glasses, which shielded the left and right eye from direct light of the left and right monitors respectively. Thus, participants could only see the reflection of the left monitor with the left eye, and the right monitor with the right eye. Shown are also the stimuli used in Experiment 1. B. The stimuli used in Experiments 2 to 4. 


\section{Design and Tasks}

The experiment contained trials of three fundamentally different conditions. There was a standard binocular rivalry after flash suppression condition (BR), a disrupted rivalry condition (DR), and a control condition (CTR). Each of these trial types commenced after the previous trial was ended by means of a dedicated key press ('space bar'). The trial started with the 'adaptation phase', in which either the triangle image or star image

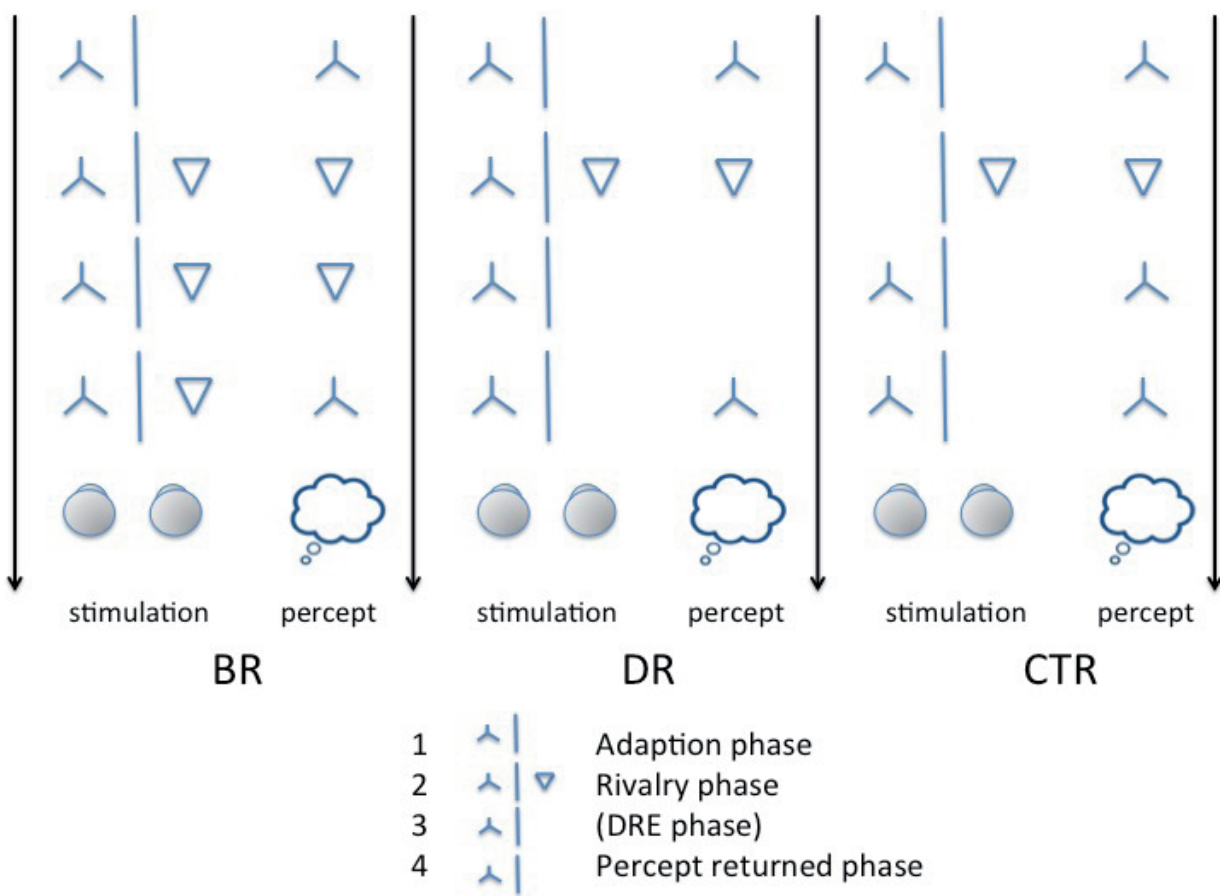

Figure 2: Main conditions and corresponding percepts

Shown separately for the main conditions of binocular rivalry (BR), disrupted rivalry (DR), and control (CTR), are the different phases of stimulation (schematic depiction of images presented to left and right eye respectively, always shown left) and corresponding percepts (schematic depiction of consciously perceived image, always shown right). For DR, the condition of interest, the different phases of a single trial are outlined with corresponding stimulation protocol inserted. Consecutively, after 1) an adaptation phase of a variable duration, there is a brief 2) rivalry phase, of variable durations of several hundreds of milliseconds, after which the rivaling stimulus is removed, and the subject may report seeing neither stimulus in the potential 3) DRE phase (=disrupted rivalry effect) of variable duration, until the stimulus element still presented to one eye return to conscious perception in the 4) percept returned phase. In the BR condition, the rivalry phase lasts much longer, namely until the first perceptual switch back to the originally adapted stimulus. In the CTR condition, the 'rivalry phase' does not really involve rivalry, as the originally adapted stimulus is removed. The participant does not notice this, as the new stimulus is dominant, but after the 'rivalry phase' the percept will immediately switch back to the remaining originally adapted stimulus. Experience dictates that the 'DRE phase' in the CTR condition is not really composed of any disappearance effect, but purely of reaction time. 
was presented to one of both eyes (starting image and starting eye were random and fully balanced over all trials) for a certain duration (=adaptation time). Adaptation time could be $250,1000,1750,2500$, or 3250 milliseconds (ms). The next phase was the 'rivalry phase'. In the BR condition, this would essentially be the last phase. One task for participants on all trials was always: 'press the keyboard NUM5 button as soon as the stimulus presented at the beginning of a trial is again fully visible' (i.e. the adapted stimulus). In all conditions, the second, competing image presented at the onset of the rivalry phase would immediately become dominant/visible. Thus, in the BR condition, participants indicated when the first perceptual switch occurred (since this is when the initial percept became again visible). In the DR condition, the rivalry phase was very short, since the second, competing image was replaced by a blank image after either $100,200,300,400$, or $800 \mathrm{~ms}$. The basis of the current report is the somewhat surprising finding that a third phase followed the rivalry phase, the 'disrupted rivalry effect' phase, in which the remaining salient image was not (fully) perceived for some time. This phase ended when subjects indicated that this percept had fully returned to visual awareness. In the third, control condition (CTR), the crucial manipulation was removal of the initially dominant, adapted image during the rivalry phase. In other words, while in the DR condition both images were presented to one eye each throughout the rivalry phase, in the CTR condition there was first one image (the adapted image) presented to one eye, then the other image to the other eye (the second, flash-suppressing image) with the adapted stimulus replaced by a blank image, then again the first (adapted) image to the first eye. Note that, subjective-perceptually, both conditions were equal up to the end of the rivalry phase. We will report, however, that in the CTR condition the remaining salient image is immediately perceived, while in the DR condition, the remaining image is not perceived for some time. See Figure 2 for a schematized visual outline of the stimulation protocol and corresponding perception in various conditions.

Participants were asked to perform a second task on each trial. They indicated which stimulus element had been the last to reappear. Participants' reports, as well as experimenters' own experience, revealed that this last reappearing element was easily identified in the BR and DR conditions (in earlier pilot experiments we experimented with larger numbers of elements, finally determining that eight elements could reliably be kept track of), but not at all on the CTR condition (as the perceptual switch here was instantaneous). On all trials, as soon as participants had indicated full percept of the adaptation image by button press, the eight stimulus elements were replaced by arrows pointing in 8 directions. These arrows indicated which buttons on the keyboard NUMpad corresponded to these visual field locations. Participants then self-paced their response on this task, ending trials with a third key press (space bar).

All trial types were fully balanced in this wholly within-subject experiment. The experiment was conducted in 2 runs in one session which each consisted of 10 blocks of trials ( 8 blocks of 50 trials and 2 blocks of 20 trials towards the end), separated by breaks in which lights were turned on in the laboratory and subjects were allowed to relax and move around. With 5 BR conditions (5 adaptation times) and 25 DR and CTR conditions (5 adaptation times x 5 rivalry times), the total number of trials per condition cell was 16 , and the total number of trials over both sessions was 880 . 
Analysis

Trials were excluded from analysis in preprocessing using custom Matlab-based preprocessing, if participants did not follow instructions properly (e.g. pressed no or wrong buttons, gave the 'location'-response before the 'percept return'-response, gave no 'location-response', etc.). Outliers were removed on the basis of individual mean reaction times (RTs) and standard deviations separately per BR, DR, and CTR conditions, by the rule that trials with RTs more than 2.5 standard deviations above the individual mean were excluded.

The design did not allow a full-model repeated-measures ANOVA (RMANOVA) (e.g. no rivalry time conditions within the BR condition), so we determined a priori which RM-ANOVAs we were interested in (always Huynh-Feldt corrected). These were condition (BR, DR, CTR) $\mathrm{x}$ adaptation time (Atime: 5 levels) and condition (DR, CTR) $x$ rivalry time (Rtime: 5 levels). Also, for the DR and CTR conditions separately we evaluated RM-ANOVAs of Atime $x$ Rtime. Follow-up analyses as reported in Results section.

To evaluate whether the dominance periods of the flashed stimulus (in BR) and durations of potential disappearance effects (DRE, in DR) were homogeneously distributed over the visual field, subjects indicated which stimulus element out of the array of 8 elements was the last to switch (BR) or re-appear (DR). The distributions of these reports were analyzed for homogeneity using subject- and condition-specific ChiSquare Tests.

\section{Results}

A repeated-measures analysis of variance (RM-ANOVA) with factors condition (Cond: $\mathrm{BR}, \mathrm{DR}, \mathrm{CTR})$ and adaptation time (Atime: 5 levels) revealed no interaction $(\mathrm{P}=0.822)$, no effect of Atime $(\mathrm{P}=0.618)$, but a main effect of Cond $(\mathrm{F}=23.9 ; \mathrm{P}<0.001)$. The average reaction times ( $\mathrm{RT}$ : indicating time to last nucleation for $\mathrm{BR}$, time to full percept return for DR, or baseline reaction time for CTR) are shown in Figure 3. Posthoc comparisons showed that all three conditions significantly differed from each other (all $\mathrm{P}<0.05$, bonferroni corrected). The slower responses on DR than CTR formally established the disrupted rivalry effect.

\section{Effects of adaptation time or rivalry time on DRE}

The RM-ANOVA with factors condition (Cond: DR and CTR) and rivalry time (Rtime) yielded a main effect of Cond $(\mathrm{F}=97.9 ; \mathrm{P}<0.001)$ and Rtime $(\mathrm{F}=30.5 ; \mathrm{P}<0.001)$, but also an interaction $(\mathrm{F}=8.9 ; \mathrm{P}<0.001)$. Follow-up $\mathrm{RM}$-ANOVAs of Rtime separately for DR and CTR showed that Rtime did significantly affect $(\mathrm{F}=17.7 ; \mathrm{P}<0.001) \mathrm{RT}$ for $\mathrm{DR}$, as well as CTR $(\mathrm{F}=19.8 ; \mathrm{P}<0.001)$. Figure 4 shows the RTs over Rtime, for DR and CTR. The effect of Rtime in the control condition seems due to the sharply increased RT for the shortest Rtime. As the onset of rivalry in effect constituted a temporal cue priming 
the response, this very short Rtime likely provided insufficient 'warning' of the upcoming required button press. To confirm this, we re-evaluated without the shortest Rtime the RM-ANOVA of Cond (DR, CTR) $x$ Rtime (4 levels). There were again main effects of Cond $(\mathrm{F}=86.3 ; \mathrm{P}<0.001)$, Rtime $(\mathrm{F}=10.3 ; \mathrm{P}=0.001)$ and an interaction $(\mathrm{F}=9.6$; $\mathrm{P}<0.001)$. Follow-up RM-ANOVAs per condition showed that there was now an effect of Rtime on $\mathrm{RT}$ in disrupted rivalry $(\mathrm{F}=19.1 ; \mathrm{P}<0.001)$, but not in the control condition $(\mathrm{F}=1.6 ; \mathrm{P}=0.229)$.

RM-ANOVAs per condition (DR, CTR) revealed no interaction between Atime and Rtime for DR $(\mathrm{P}=0.293)$ but an interaction for $\mathrm{CTR}(\mathrm{F}=2.5 ; \mathrm{P}<0.05)$. This was again due to the effect for the shortest Rtime, as there was no interaction when removing the $100 \mathrm{~ms}$ Rtime from the analysis $(\mathrm{F}=1.4 ; \mathrm{P}=0.251)$. When we re-analyzed the effect of Atime on DR and CTR trials excluding (for reasons given above) the

\section{Time to full initial percept return}

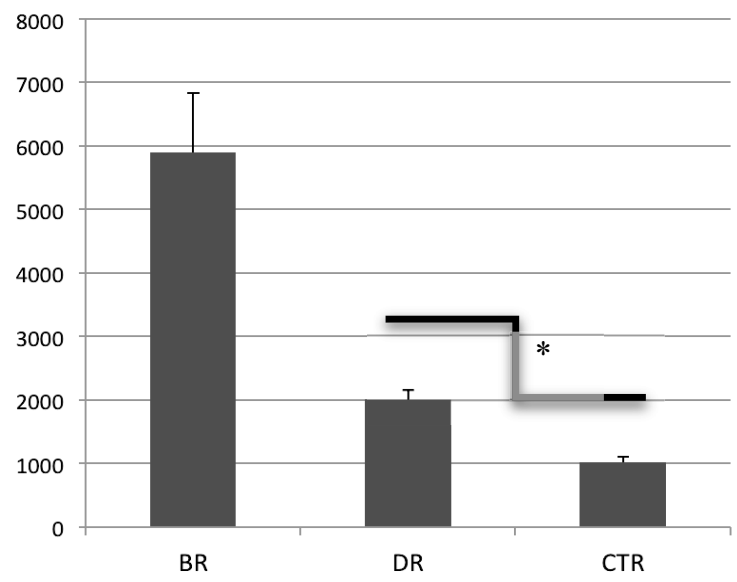

Figure 3: Reaction times in main conditions

For condition BR (binocular rivalry), reaction times (RT vertical axis) reflected the time from rivalry (started with flash suppression after an adaptation phase) to the first full perceptual switch back to the original adapted stimulus. For condition DR (disrupted rivalry), RT reflected the time from the end of the rivalry phase to the start of the percept return phase, or in other words the time from removal of the flashed rivaling stimulus to reported full percept of the only remaining stimulus. RT for condition CTR (control condition) reflects the same, except that there was not really any disappearance effect - thus this condition is a form of baseline: if the RTs in DR were larger than in CTR, this indicates that the remaining stimulus was not immediately visible again. Indeed, as indicated by the asterisk, RTs in DR were significantly longer than in CTR, establishing the disrupted rivalry effect (DRE). shortest Rtime, there was a significant effect of Atime $(\mathrm{F}=4.2 ; \mathrm{P}<0.01)$, but since this did not interact with condition (DR, CTR; $\mathrm{P}=0.818)$ the interpretability was limited.

One thing of note was the duration of the effect. Figure 5 shows histograms of the RTs over all subjects (A) or one subject (B). There were a limited number of trials in the control condition where participants took a long time to respond (presumably not following instructions, or not pressing the response key firmly enough and pressing again some time later). In contrast, in the disrupted rivalry condition there were a large number of trials in which the stimulus had disappeared from perception for seconds on end, with a mean duration of over 2 seconds, but many instances of much longer durations. Moreover, in the General Discussion of Part I, we discuss why our estimation 
in these experiments is likely very conservative.
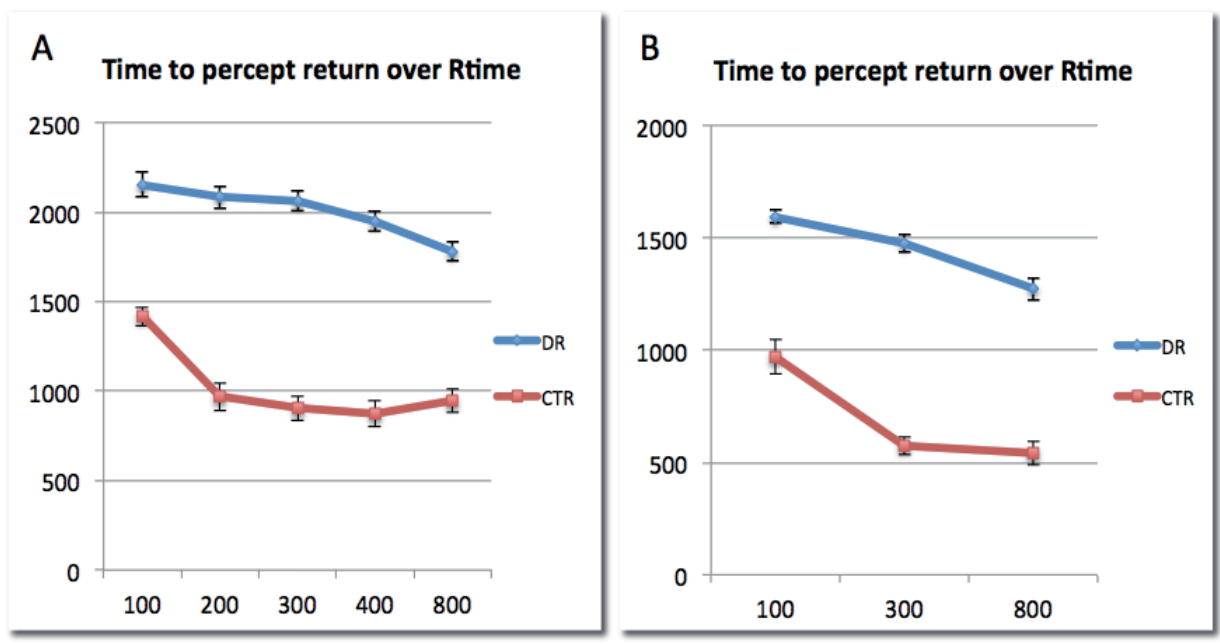

Figure 4: The duration of DRE effects over rivalry time

A. Experiment 1. Shown separately for DR and CTR conditions, are the RTs (vertical axis) for various durations of the rivalry phase (rivalry time; Rtime). For DR, there is a general linear trend towards decreased DRE duration with increased rivalry time. For CTR, the shortest Rtime of $100 \mathrm{~ms}$ resulted in peak reaction time, but otherwise the downward trend was not mirrored. Thus, in disrupted rivalry, the disappearance effect is larger with briefer flashes of a rivaling stimulus. B. Same as in A., but for Experiment 2 - with single stimulus elements instead of stimulus element arrays. Within-subject error bars (Cousineau, 2005)

\section{Non-uniform DRE duration across the visual field}

Van Ee (2011) recently showed that binocular rivalry processes are not uniform across the visual field, asking participants to indicate after flash suppression which stimulus element out of a peripheral array switched back first to a previously adapted rivalling stimulus. The distributions of these 'first percept-switch nucleations' were not homogenous, indicating the involvement of local processes. Here, we aimed to replicate this finding for the binocular rivalry (BR) condition. However, we extended it by asking participants for the 'last nucleation' instead of the first. Moreover, in the same participants we evaluated the same hypothesis of inhomogeneous distributions of these last 'nucleations' for the disrupted rivalry effect (DRE). If DRE is fully subserved by higher-order (competition or other) mechanisms, the distribution should be homogeneous over the eight locations. Participants reported no problems with this task, as it was generally subjectively clear which element last switched (BR) or reappeared (DR). 
For BR, the number of trials may have been insufficient to replicate in all subjects the inhomogeneous distribution of dominance periods over the visual field, although for six out of twelve participants the distribution was significantly (4) or marginally significantly (2) inhomogeneous. For DR, however, eleven out of twelve participants demonstrated a significantly inhomogeneous distribution. The distributions are displayed in Figure 6. These results were so strongly significant that they survived a full bonferroni-correction for twelve tests. Moreover, Figure 6 makes clear that the distributions were very dissimilar between participants, ruling out that potential constant differences between visual field locations (e.g. monitor- or stimulus-related) caused the inhomogeneous distributions of DRE. This strongly suggests that the neuronal mechanisms underlying DRE involve at least in part local competition processes.

Histograms of durations until percept return
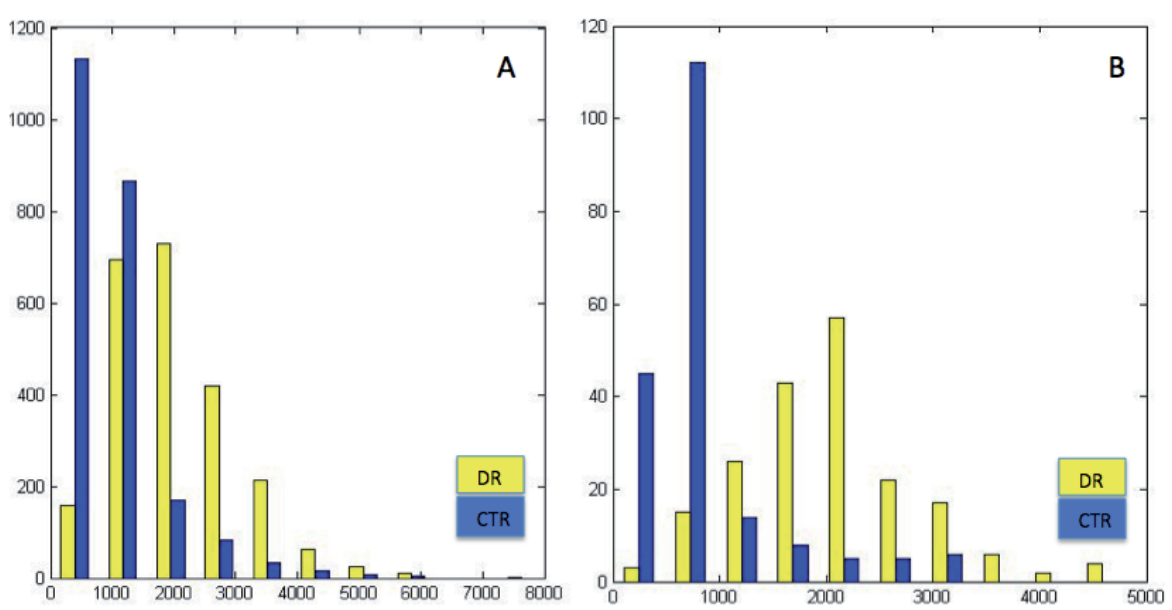

Figure 5: distributions of reaction times in main conditions DR and CTR

A. Shown in blue are binned counts of reaction times (RTs) in the control condition CTR. Shown in yellow is the same, but for the DR condition. The few trials with long reaction times in the control condition are likely due to participants either taking a mini-break (in discordance with instructions) since the required button press would initiate the next trial, or not pressing the response button firmly enough and pressing it again a few seconds later. While such factors may also be involved in the DR condition, there are substantially more trials with reaction times in the order of several seconds in this condition. This difference indicates that the DRE can last for seconds on end. B. same as in A, but for one representative participant. 


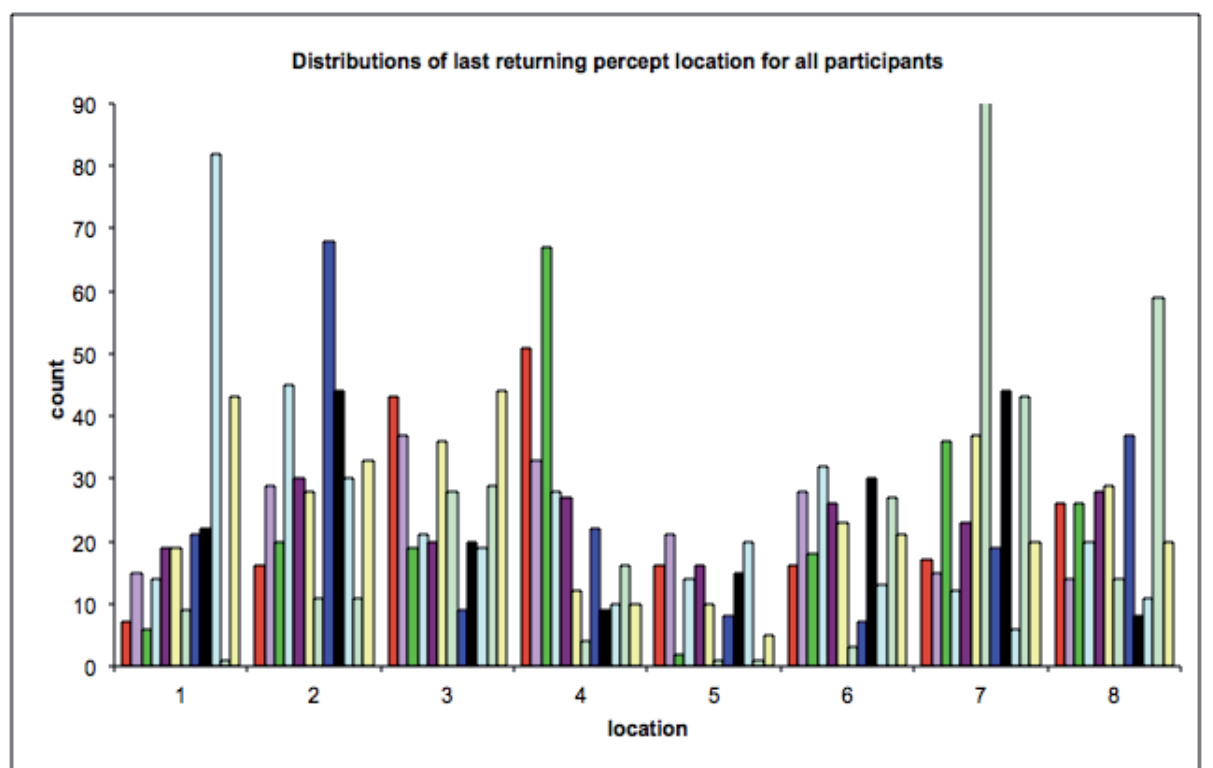

Figure 6: distributions of last returning percept location for all participants Counts (vertical axis) of the numbers of trials in which each of 8 locations (horizontal axis) contained the last stimulus element to return to perception, as indicated by participants on each trial. Different participants are color-coded. Most important here is that 1) the distributions for individual participants seem to be inhomogeneous, and 2) the distributions are very different between participants - excluding potential causes of inhomogeneity due to stimulus or computer monitor characteristics.

\section{Discussion}

It is clear that the disrupted rivalry effect (DRE) is significant and powerful in a sample of participants familiarized with the effect in a few trials but otherwise completely naive and untrained in binocular rivalry paradigms. This effect decreased with rivalry time. The effect is inhomogeneous across the visual field, suggesting that it involves (in part) local rivalry processes. The effect of adaptation time was however unconvincing: weak in terms of effect size and statistically underpowered. Although there was a main effect of adaptation time, it did not interact with condition. However, the adaptation times were short, the stimuli constituted a wide array of elements covering the visual field, and trial lengths directly depended on adaptation time. The latter consideration might confound the results, given our informal observation that any eye movement in the disrupted rivalry effect phase immediately and completely abolishes the effect (i.e. the remaining salient stimulus immediately and fully becomes dominant again), as should be expected of visual suppression phenomena (see General Discussion below). In following experiments we addressed these considerations. 


\section{Experiment 2: the disrupted rivalry effect in one fully attended}

\section{visual field location}

\section{Introduction}

Having established the DRE in Experiment 1, we here made one major change in the design: instead of presenting an array of competing visual elements covering all four visual field quadrants, we presented only one pair of competing visual elements at one of four visual field locations on any given trial (the four possible locations corresponding to the four visual quadrants). This fully eliminates any possible influences of attention (or attention bias) during the trial or over the visual field. In this setup we thus stripped the DRE to the barest of bones: the location where a salient visual stimulus is known to exist is fully attended to throughout the trial.

\section{Materials and Methods}

\section{Participants}

Nine participants were tested in two sessions per subjects. Eight of these were fully naive to the goals and content of the experiment, and all were completely inexperienced in binocular rivalry paradigms. All had normal or corrected-to-normal vision and provided written informed consent.

\section{Stimuli, Tasks, and Design}

Blank images were unchanged, the elements were still triangles and stars, but now there were four 'triangle' and four 'star' images. They were fully symmetrical, with the triangle 'pointing' to the fixation dot in all four visual field locations (one in each visual quadrant). The stimuli used in this and following experiments are shown in Figure 1B.

The task was again to 'press the button when the initial percept fully returns'. The second task of experiment 1 , indicating which element returned last, was dropped since there was only one element per trial.

Again BR, DR and CTR conditions (factor 'Cond') were implemented, with the addition of factor 'element position' (Pos), and a reduced number of adaptation times (Atime: now 250, 1750, and $3250 \mathrm{~ms}$ ) and rivalry times (Rtime: now 100, 300, $800 \mathrm{~ms}$ ). Trials were fully balanced between the monitor (eye) that was the adapting stimulus, and the stimulus (star/triangle) that was adapted to. The fully within-subject design thus included conditions Cond, Atime, Rtime, Pos, with 8 trials per condition cell per participant, leading to a total of 672 trials per participant separated into two sessions on different days (614 trials for one participant). 


\section{Analyses}

Outlier trials were removed from analysis by the rule that RTs per condition (as in Experiment 1) could not exceed 2.5 subject- and condition-specific standard deviations above the mean. 34 trials ( $\sim 0.5 \%$ of total) had unrealistic RTs below $200 \mathrm{~ms}$ and were also removed.

Repeated-measures ANOVAs (Huyhn-Feldt corrected) determined a priori were an analysis containing factors Cond, Atime, Pos. For DR and CTR trials, a RMANOVA containing Cond, Atime, Rtime, Pos was performed. Follow-up tests were implemented as indicated in the Results section.

\section{Results}

A repeated-measures ANOVA (RM-ANOVA) with factors Cond, Atime, Pos revealed a strong main effect of Cond $(\mathrm{F}=90.6 ; \mathrm{P}<0.001)$ and statistical trends for Atime $(\mathrm{F}=3.8$; $\mathrm{P}=0.067)$ and for $\mathrm{Pos}(\mathrm{F}=3.6 ; \mathrm{P}=0.076)$. Follow-up pairwise comparisons showed that RTs significantly differed between all three conditions (BR, DR, CTR) (all Ps $<0.001$, bonferroni-corrected). There were no interactions for Atime with other conditions, but the effect of stimulus element position (Pos) was dependent on condition (Cond) $(\mathrm{F}=4.2 ; \mathrm{P}<0.05)$. Follow-up RM-ANOVA analyses of Pos per level of Cond showed that $\mathrm{Pos}$ had a significant effect on $\mathrm{RT}$ in $\mathrm{BR}(\mathrm{F}=5.2 ; \mathrm{P}<0.05)$, and in CTR $(\mathrm{F}=7.9$; $\mathrm{P}<0.05)$, but not in DR $(\mathrm{P}=0.46)$. An effect of $\mathrm{Pos}$ for $\mathrm{BR}$ suggests that on the group level, there might be some consistent differences in dominance durations across the visual field (e.g. Chen \& He, 2003). However, since there was also an effect of Pos for the control condition CTR (in which no effects actually occur), there seemed to be a consistent bias in response speed (possibly attentional bias), making other effects of Pos difficult to interpret. Since the design was fully balanced and there was no effect of Pos for DR, Pos was left out of further analyses.

The RM-ANOVA with factors Cond (DR and CTR), Atime and Rtime revealed a significant main effect of Rtime $(\mathrm{F}=27.2 ; \mathrm{P}<0.001)$, but moreover an interaction of Rtime with Cond $(\mathrm{F}=4.9 ; \mathrm{P}<0.05)$. Follow-up analysis showed that Rtime had a strong significant effect in DR $(\mathrm{F}=24.6 ; \mathrm{P}<0.001)$, and also an effect in CTR $(\mathrm{F}=7.0 ; \mathrm{P}<0.05)$. Figure $4 \mathrm{~B}$ shows the pattern of $\mathrm{RT}$ over Rtime for both $\mathrm{DR}$ and $\mathrm{CTR}$ conditions. It becomes clear that, as in Experiment 1, the shortest Rtime in the control condition is 'too short', in the sense that participants are much slower to respond. The interaction between Rtime and Cond shows that in the disrupted rivalry condition, rivalry time has an effect over and above this baseline effect. To confirm, a 2x2 RMANOVA leaving out the shortest Rtime yielded a significant interaction between Cond (DR, CTR) and Rtime $(300,800)(\mathrm{F}=7.7 ; \mathrm{P}<0.05)$. Follow-up comparisons showed that Rtime now did affect $\mathrm{RT}$ in disrupted rivalry trials $(\mathrm{t}(8)=3.5 ; \mathrm{P}<0.05$ - 2-tailed, bonferroni-corrected), but not in the control condition ( $\mathrm{P}=0.217$ uncorrected). In short, in disrupted rivalry, longer rivalry durations lead to shorter-lasting disappearance effects. In contrast to experiment 1 , there was no main effect (or interaction with Cond) of Atime when excluding data with the shortest Rtime. 


\section{Discussion}

Somewhat surprisingly, the DRE in fully naive and untrained subjects was still present and quite strong in this experiment. Subjective reports suggested that with only one stimulus, it was rather difficult to maintain fixation. There is a non-central location where 'something' exists, yet the subject does not see it. This leads to a strong impulse to make a saccade to the location. Yet, subjects apparently managed well enough to maintain fixation, as shown by the presence of DREs with durations up to several seconds. Again, the effect of adaptation time was unconvincing. This could have three reasons at this point. One, perhaps adaptation time does not affect the DRE. We should not assume this option for the moment, since a previously demonstrated effect of adaptation time (van Ee, 2011) in the binocular rivalry condition was also not replicated here, and the contribution of local adaptation build-up to binocular rivalry dominance durations is widely accepted (Alais, Cass, O'Shea, \& Blake, 2010; Kang \& Blake, 2010; van Ee, 2011) suggesting that our design or subject sample size lacked sensitivity to detect the putative effect. Alternatively, perhaps the propensity to make an eye movement becomes stronger with time, leading subjects to make eye movements more often with longer adaptation times. Or, perhaps our stimuli and design choices would require longer adaptation times to clearly demonstrate the influence of local adaptation build-up. We proceeded in follow-up experiments 3 and 4 to further probe the effect of adaptation time on DRE duration, addressing both of the latter possibilities. 


\section{Experiment 3: single-element paradigm with fixed trial durations}

\section{Introduction}

One explanation of small or no effects of adaptation time on DRE might be the increasing trial (and required fixation) duration with increasing adaptation time. Longer required fixation might mean proportionally more unintentional saccades. Thus, the increase in DRE due to longer adaptation might potentially be counteracted by the decrease in average DRE due to more frequent eye movements with longer adaptation times. Since our setup did not allow the tracking of eye movements, we performed an experiment in which we fixed trial duration and thereby fixation time, using again the single-element stimuli from experiment 2.

\section{Material and Methods}

\section{Participants}

Fifteen participants participated in this experiment. Fourteen of them were naive to the goals and content of the study, and all were inexperienced with binocular rivalry. They had normal or corrected-to-normal vision and provided written informed consent.

\section{Stimuli, tasks, and design}

The stimuli and tasks were identical to experiment 2. Again BR, DR, and CTR conditions were implemented. We increased the adaptation times with $250 \mathrm{~ms}$ (now $500,2000,3500 \mathrm{~ms})$. The rivalry times were changed to $150,350,550 \mathrm{~ms}$.

With factors Cond (BR, DR, CTR), Atime (3 levels), Rtime (3 levels), Pos (4 levels), fully balanced over monitors (eyes) and adapted stimulus, 4 trials per condition cell were measured per participant, leading to a total of 336 trials per participant measured in one session.

The most important change in experimental design was the fixation of trial duration. While in other experiments the fixation dot and frame ('blank image') were continuously present if no stimuli were presented, here trials ended with a full disappearance (and thus subsequent reappearance to start new trials) of the fixation dot and frame. This clearly indicated the start of a trial. Then, the time to the end of the rivalry phase (i.e. the onset of a potential disrupted rivalry effect phase) was kept constant. Concretely, a variable initial fixation phase (with only two blank images presented) added up with adaptation time and rivalry time to a total of $5000 \mathrm{~ms}$. 


\section{Analyses}

Trials were excluded if instructions were not followed properly, or if RTs were unrealistically lower than $200 \mathrm{~ms}$ ( 91 trials, $=1.8 \%$ of total) and outliers with over 2.5 standard deviations (subject- and condition-specific) above the mean. Repeatedmeasures ANOVAs (RM-ANOVA) were used to evaluate the a priori determined analyses: RM-ANOVA of Cond (BR, DR, CTR) x Atime $\mathrm{x}$ Pos and RM-ANOVA of Cond (DR, CTR) x Atime x Rtime (Huynh-Feldt corrected). Follow-up tests and additional analyses as reported in Results section.

\section{Results}

The full repeated-measures ANOVA (RM-ANOVA) with factors Cond (BR, DR, CTR), Atime, and Pos revealed main effects of Cond $(\mathrm{F}=119.6 ; \mathrm{P}<0.001)$ and Atime $(\mathrm{F}=5.9 ; \mathrm{P}<0.01)$, but no interactions. The interaction with Pos found in Experiment 2 was not replicated. While an effect of adaptation time was hypothesized, the expectation was that Atime would affect specifically BR and DR, but not CTR. Or, at least, that the effect would be in some way specific to the rivalry conditions.

The RM-ANOVA with factors Cond (DR and CTR), Atime and Rtime yielded a main effect of Cond $(\mathrm{F}=62.8 ; \mathrm{P}<0.001)$, Atime $(\mathrm{F}=11.1 ; \mathrm{P}=0.001)$, and Rtime $(\mathrm{F}=14.6 ; \mathrm{P}=0.001)$. Moreover, there was a trend for interaction between Atime and Rtime $(\mathrm{F}=2.4 ; \mathrm{P}=0.06)$ and a significant interaction between Rtime and Cond $(\mathrm{F}=4.6$; $\mathrm{P}<0.05)$. Follow-up analysis showed that Rtime had a significant effect both on disrupted rivalry trials $(\mathrm{F}=9.6 ; \mathrm{P}=0.001)$ and control trials $(\mathrm{F}=3.1 ; \mathrm{P}<0.01)$. These results largely mirrored the findings in Experiment 2, with the shortest Rtime leading to a sharp reaction time increase in the CTR condition, but an effect of Rtime in DR trials over and above this.

\section{Discussion}

This experiment confirmed again the existence of DRE, using single-element stimuli. Results resembled the previous experiment. Most importantly here, the fixation of trial duration did not increase markedly the effect of adaptation times on the duration of DRE. There was a main effect, but the hypothesized interaction between adaptation time duration and condition was not obtained, so differences in fixation time could be excluded as explanation for the lack of adaptation time effects on DRE in previous experiments. 


\section{Experiment 4: increased adaptation times do affect the duration of}

\section{DRE.}

\section{Introduction}

Having excluded the possible confounding effect of fixation duration by fixing trial length, we proceeded to sharply increase the durations of the adaptation phase. With adaptation up to 21 seconds, any putative effect on DRE duration should surely become apparent. Also, the effects of adaptation time on reaction times in the control condition could plausibly disappear, given the more widely spaced and unpredictable rivalry onsets (i.e. end of adaptation). The binocular rivalry condition was omitted to save time, focusing this experiment solely on the potential effect of adaptation time on the duration of the disappearance effect DRE.

\section{Materials and Methods}

\section{Participants}

Five participants returned four times for a total of 20 measurements. This was necessary to obtain sufficient trials with trial durations of markedly increased duration. All had normal or corrected-to-normal vision and provided written informed consent.

\section{Stimuli, tasks and design}

The stimuli were identical to the previous single-element experiments. The BR condition was omitted, to constrain the total number of required trials. Thus DR and CTR conditions with 6 adaptation times (1000, 5000, 9000, 13000, 17000, $21000 \mathrm{~ms})$ and 4 rivalry times $(200,500,800,1100 \mathrm{~ms})$ were implemented. A total number of 8 trials per condition cell per session (i.e. over all sessions 32 trials per condition cell per subject) were collected (i.e. per session 192 trials in total).

The task was changed slightly, in that participants were asked to press and hold the keyboard button whenever the initially perceived stimulus was not perceived. This could thus be anywhere during the adaptation, rivalry, or disrupted rivalry effect phases, for any duration.

\section{Analyses}

Trials in which instructions were not properly followed or RTs of unrealistic values (less than 200ms) were removed prior to analysis (241 trials; $6.3 \%$ of total). Outliers 
were removed by the criterion of 2.5 standard deviations above the individual condition-specific mean. A repeated-measures ANOVA (RM-ANOVA) was performed with factors Cond (DR, CTR), Atime (6 levels), Rtime (4 levels), with follow-up tests as indicated in Results section (Huynh-Feldt corrected).

\section{Results}

The full-model RM-ANOVA containing factors Cond (DR, CTR), Atime and Rtime revealed significant main effects of Cond $(\mathrm{F}=15.6 ; \mathrm{P}<0.05)$, adaptation time $(\mathrm{F}=3.3 ; \mathrm{P}<0.05)$ and rivalry time $(\mathrm{F}=11.4 ; \quad \mathrm{P}<0.05)$. However, while Rtime did not interact with other factors, the hypothesized interaction of Cond $\mathrm{x}$ Atime was strongly significant $(\mathrm{F}=6.6 ; \mathrm{P}=0.001)$. This indicates that adaptation time had a specific effect on one of the two conditions.

Follow-up RM-ANOVA of the effects of Atime and Rtime per condition showed that this was due to a significant effect of Atime on RT in disrupted rivalry trials $(\mathrm{F}=5.4 ; \mathrm{P}<0.01)$, while Atime had no effect in the baseline condition $(\mathrm{P}=0.882)$. It is thus clear that the duration of adaptation time does indeed affect the duration of the disappearance effect in the disrupted rivalry paradigm, provided a wide enough range of adaptation durations is implemented. Figure 7 displays the RTs over adaptation time durations for DR and CTR trials separately. In contrast with the flat baseline over Atime for the

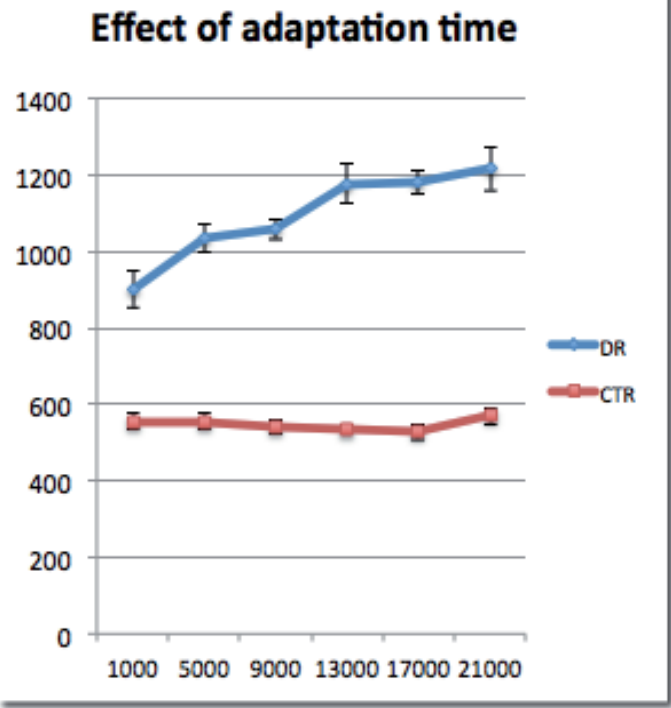

Figure 7: effect of adaptation phase duration on DRE

Shown separately for conditions DR and CTR are the reaction times (RT: vertical axis) over the six levels of adaptation phase duration (adaptation time: Atime, in milliseconds on horizontal axis) in Experiment 4. While there was no effect of Atime on reaction times in the control condition CTR, there was a clear increase of the DRE with increasing adaptation time. This increase was linear. Within-subject error bars (Cousineau, 2005). control trials, there seems to be a linear increase in RT in disrupted rivalry trials with increasing adaptation time. Indeed, polynomial contrasts confirmed that the effect of Atime was significantly linear $(\mathrm{F}=8.6 ; \mathrm{P}<0.05$ - higher-order contrasts not significant).

\section{Discussion}

In previous experiments, we did not find a significant effect of the duration of adaptation time on the duration of the disappearance effect (DRE) in the disrupted 
rivalry paradigm. However, if the DRE is related to low-level competition processes, putting it in the family of binocular rivalry phenomena, an effect of adaptation time is expected. Previous work with a range of adaptation times similar to experiments 1-3 did find an effect of adaptation time on dominance durations (our 'BR' condition) (van Ee, 2011). As we did not replicate this finding unambiguously in experiments $1-3$, though expecting it to exist based on previous work (Alais, et al., 2010; Kang \& Blake, 2010; van Ee, 2011), we could not draw strong conclusions about the role of adaptation time in DRE. Having excluded possible causes for the previous null result in experiments 23 , we here sampled a much broader range of adaptation times and focused the experiment on manipulating just this variable. The clear positive finding confirms and further constrains our initial interpretation that the DRE phenomenon is probably within the family of binocular rivalry, and involves (at least in part) local competition.

\section{General Discussion}

In a series of experiments we presented a phenomenon arising in a binocular rivalry setup, that - to the best of our knowledge - constitutes a new and surprising effect. Binocular rivalry involves a stochastically varying percept due to competition between two stimulus representations. Yet, we here showed that a salient and previously dominant stimulus presented to one eye can actually be suppressed for durations of up to several seconds without any kind of salient stimulus presented to the opposite eye. This effect was found when rivalry was quickly disrupted after flash suppression, thus we dubbed it the 'disrupted rivalry effect' (DRE). The characteristics of this DRE revealed that it persists even when attention is fully focused on the stimulus location, that its duration increases linearly with the adaptation duration of the previously dominant stimulus, decreases with rivalry time, and that its distribution across the visual field is inhomogeneous.

These findings may be explained by a simplified model of binocular rivalry in which the representations of two competing images are in mutual inhibition, if one assumes that the offset of one of the two sensory streams boosts the inhibition to the other representation. That the onset of a sensory stream boosts this inhibition has long been known as 'flash suppression' (Wolfe, 1984), although it has been suggested that the neuronal mechanisms underlying the inhibition from binocular rivalry may not be identical to those underlying the inhibition from flash suppression (Tsuchiya 2006). It was previously shown that visual transients can induce perceptual switches (Kanai, et al., 2005; Walker \& Powell, 1979; Wilson, Blake, \& Lee, 2001). And visual change, rather than mere presence of luminance sensory input, may be the most interesting sort of visual signal to early regions of the human visual system (Macknik \& Livingstone, 1998; Roland, 2010). Indeed, while standard binocular rivalry involves suppression periods of seconds, continuous flash suppression, in which one stable image presented to one eye is suppressed by continuously flashed images to the other eye, can result in suppression periods of several minutes (Tsuchiya \& Koch, 2005). Moreover, the depth of suppression in such continuous flash suppression is much stronger (Tsuchiya, Koch, Gilroy, \& Blake, 2006). That the offset of a stimulus may have strong inhibitory consequences was shown in visual masking experiments (Macknik \& Livingstone, 
1998; Macknik, Martinez-Conde, \& Haglund, 2000). Macknik et al. (2000) showed that the 'temporal' edges (i.e. onset and offset) of visual masks had the strongest masking effects. In the paradigm of disrupted rivalry, then, flash suppression by competing stimulus onset may thus quickly be followed by a second 'flash suppression' by competing stimulus offset.

In our paradigm, 'eye' and 'representation' were not disentangled, as for instance in Logothetis et al.(1996). It is thus difficult to say on what level of the visual system competition takes place in our experimental setting. On the one hand, the stimuli are of a size and form that do not preclude contribution of a higher-order competition mechanism (e.g. when superimposed, the triangle and star overlap minimally). On the other hand, if the situation during the disrupted rivalry effect phase (i.e. one eye is presented with a salient high-contrast stimulus and the other eye is presented with homogenous black background) is conceived of as binocular rivalry between a salient stimulus and homogenous black, it is difficult to believe that a 'representation' of homogenous black could suppress a distinct and salient shape for very long.

We thus interpret the DRE in terms of mutual inhibition between eye-channels. The quick flash-suppression of an already somewhat adapted eye-channel followed by a second flash suppression by stimulus offset may lead to a double-inhibition-boost to the fatigued eye-channel. Moreover, the 'flashed' eye-channel may have gained some relative strength from the double transient, which may persist for a little while even though there is no real further stimulation of the channel. The lack of transient changes in the previously dominant eye-channel means that the channel takes rather long to recover from this double-inhibition even if it is no longer receiving any significant inhibitory signals from a competing percept. Of course, eventually the percept returns, whether due to natural recovery of the channel or due to (micro-)saccades which constitute a visual transient and result in different neuronal ensembles receiving the sensory input.

The inhomogenous distribution of DRE effect sizes over the visual field suggests that this postulated mechanism takes place early in the visual system at the local level (i.e. in accordance with Whittle et al. (1968): local rivalry zones, see (Blake, 2005). The double-inhibition hypothesis on the level of eye-channels also explains why the effect is wholly absent in the control condition. After all, in the control condition, when the adapted stimulus is removed during the 'rivalry' phase and re-presented, this eye-channel has received also a stimulus offset, and again onset, to counter the inhibitory signals from the second eye-channel.

Figure 8 shows schematically why in this framework the duration of adaptation phase and rivalry phase should affect DRE in the way that, based on experiments 1 through 4 , indeed they do. With increasing adaptation time, the relative advantage of the adapted eye-channel at the moment of flash suppression is weaker, and the difference at the onset of rivalry phase is greater, which carries through to the DRE (Figure 8A). The double-inhibition account itself is schematized in Figure 8B. Figure $8 \mathrm{C}$ shows why DRE is shorter with increasing duration of the rivalry phase. See figure caption for further details.

Recent work has shown that visual after-images of gratings can engage in binocular rivalry with each other (Gilroy \& Blake, 2005). Moreover, when matched for 
subjective intensity, after-images can engage in rivalry with (and become dominant) real images (Bartels, Vazquez, Schindler, \& Logothetis, 2011). While subjects did not report this, and while to us the impression was not overwhelming, it is possible that the flashed competing image resulted in an after-image. In this scenario, the after-image in one eye channel could have been engaged in binocular rivalry with the real image in the other channel. If so, not many of our interpretations would change. However, it seems unlikely to us to fully explain DRE, given the huge difference in objective and subjective intensity between the present real image and the potential competing after image. The flashed real image that should then have led to the after-image would in any case have been much shorter in duration than the after image itself - since the DRE on particular trials lasted for several seconds. Lastly, since after-images increase in strength and duration with presentation time of the eliciting real image, increasing rivalry time should lead to increasing DREs if after-images explain the DRE phenomenon. This was not observed; the opposite was observed. A decrease in DRE with increasing rivalry times is better explained by the double-inhibition hypothesis, since the inhibition boosts from flashed stimulus onset and offset are further apart and DRE is measured on relation to rivalry stimulus offset.

These results show that the brain can be 'fooled' into failing to represent a very strong, salient, present stimulus - which participants KNOW to be present yet cannot see - for several seconds on end. We strongly feel that the duration of DRE has been severely underestimated here, by the use of untrained fixators not used to binocular rivalry paradigms and not personally invested in the experiments. Indeed, on certain trials, the effect could last for several seconds. Some of the authors of this paper, themselves trained in fixation maintenance, experienced the effect for multiple seconds as a rule rather than exception, in informal observations. This also suggests that the effect may have methodological value. For instance brain-imaging studies on the neural correlates of consciousness could well use paradigms in which stimuli are presented yet not perceived (see Prologue). The current paradigm might allows with trained participants, the presentation of salient stimuli for seconds on end with accompanying conscious percept. It has methodological advantages in this respect, compared to for example continuous flash suppression, because it does not require the concomittant presentation of (neurally noisy) salient competing stimuli. Rather, it seems to be a fully inherent suppression of conscious perception without any real competing stimulus.

The results presented here also raise intriguing further questions. The doubleinhibition hypothesis suggests that, for example, stimulus offset by itself in binocular rivalry should have an inhibitory influence. Also, one may wonder how DRE is affected by a gradual, rather than abrupt, offset of the rivaling (flashed) stimulus. Presumably, this would decrease the effect duration? Such questions are addressed in the next set of experiments, reported in chapters I.2-I.4. 

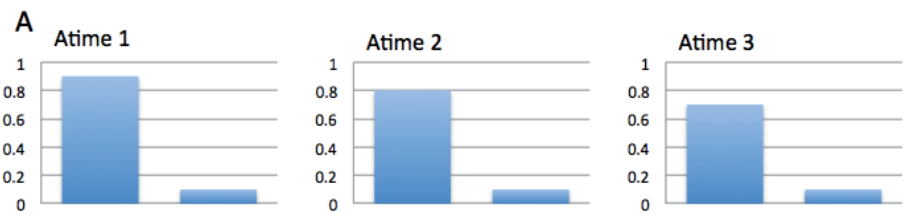

Adaptation phase
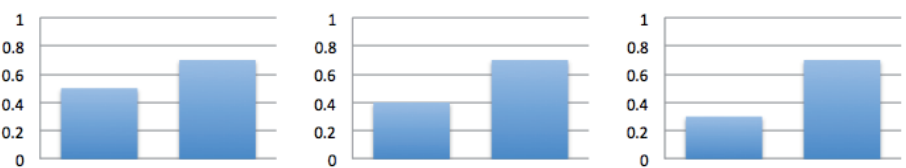

Rivalry phase onset
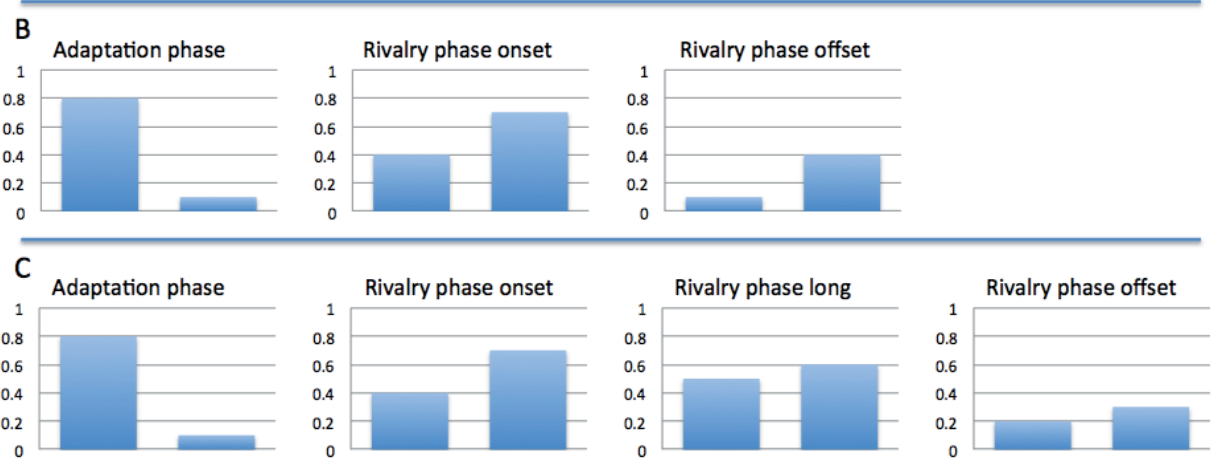

Figure 8: hypothesized mechanisms behind double-inhibition model

A. Shown schematically are the relative strengths of the left eye-channel (left blue bar in all graphs) and right eye-channel (right blue bar in all graphs), at two stages in a disrupted rivalry trial (top-three charts reflect adaptation phase, bottom-three charts reflect the situation at rivalry phase onset, or immediately at flash suppression), for three increasing levels of adaptation time (Atime1 left charts, Atime 2 middle charts, Atime 3 right charts).

Fundamental in this framework is that the eye-channel with the highest bar is currently dominant. The mutual inhibition framework dictates that, ceteris paribus, the dominant representation loses strength, while the suppressed representation gains strength, which here means that high bars will over time become lower, and low bars will become higher. Assuming (somewhat simplistically) that flash suppression by new inputs to the right eyechannel constitutes a fixed increase for the right bar and decrease for the left bar, figure A shows that with increasing Atime, the resulting relative advantage for the right eye-channel after flash suppression (at the onset of the rivalry phase) will be larger. If the doubleinhibition account of DRE is correct, this should carry over to the DRE duration.

B. Same as in A., but now the left chart reflects the adaption phase, the middle chart the situation as rivalry phase onset, and the right chart the situation after removal of the second (flashed) stimulus. Our interpretation assumes that the offset of the second, dominant stimulus, by virtue of constituting a visual transient, has two effects: 1) it boosts somewhat the right eye-channel, which is dominant at the time - this results in a relatively higher strength for this channel, although it is lower than previously as there is now no significant input, 2) it inhibits even further the left eye-channel, which was already inhibition by the flash suppression. This contribution thus essentially forms a second flash-suppression by offset.

C. Shown is why increasing rivalry time negatively affects the duration of DRE. Again the left chart reflects the adaption phase, the second chart reflects the onset of the rivalry phase. But as the flashed stimulus is dominant for longer before removal of the stimulus, the relative difference in strength between both eye-channels is reduced. Thus, at the offset of the rivalry phase (onset of DRE phase), given the same inhibitory boosts outlined under B., the right 76 eye-channel has less of a relative advantage to begin with, and the DRE will last shorter. 


\title{
Characterizing the Disrupted Rivalry
}

\author{
Effect (I): the prevalence and duration
}

\section{of disappearing stimuli under various}

\author{
conditions
}

Related publication(s):

De Graaf, TA, van Ee, R*, Goebel, R, Sack, AT (in preparation). Manuscript in preparation

* Raymond van Ee

1) Department of Physics of Man, Helmholtz Institute, Utrecht University, Utrecht 3584 $\mathrm{CH}$, The Netherlands

2) Department of Brain, Body, Behaviour, Philips Research Laboratories, Eindhoven 5656 AE, The Netherlands

3) Department of Experimental Psychology, Leuven University, Leuven B-3000, Leuven, Belgium 


\begin{abstract}
In binocular rivalry settings, flash suppression involves the immediate dominance of a fresh stimulus presented to one eye over a previously adapted competing stimulus presented to the other eye. We recently showed that quick removal of the flashed stimulus does not revert conscious perception back to the only remaining salient stimulus until seconds later. This 'disrupted rivalry effect' (DRE) was interpreted as an instantiation of rivalry between two eye-channels. Yet, also without flash suppression or even rivalry, peripheral stimuli are known to disappear from conscious perception, for example in 'Troxler fading'. To examine possible roles, relations or contributions of rivalry and fading to DRE, we measured the prevalence and durations of disappearing stimulus elements with varying eccentricity on various conditions: 1) binocular rivalry, 2) disrupted rivalry, 3) 'fading' with 1 stimulated eye, 4) fading with both eyes stimulated. Stimulus elements as used in this and previous disrupted rivalry experiments did disappear spontaneously from conscious perception in the 'fading' conditions. But this happened less frequently, and with strongly reduced durations, as compared to the disrupted rivalry condition. Thus, while mechanisms other than the hypothesized 'double-inhibition' model may contribute to the DRE, they cannot fully explain it.
\end{abstract}




\section{Introduction}

Binocular rivalry is a research paradigm in which incompatible images are presented to both eyes. Rather than fusing the images, the brain selects one of the stimuli for perception. However, after a delay that depends on cognitive/perceptual state (Brascamp \& Blake, 2012), stimulus properties (Levelt, 1965), and visual field location (Chen \& He, 2003), the percept will switch to the other image. It appears that the two representations (of both images) are in continuous competition, vying for access to conscious awareness (Alais, et al., 2010; Blake \& Logothetis, 2002; Tong, et al., 2006).

In the previous chapter, we established a potentially new phenomenon in the family of binocular rivalry, dubbed the disrupted rivalry effect (DRE). This effect consisted of the failure of a very salient stimulus to reach conscious awareness for seconds on end without any true competition. To achieve DRE, a stimulus was presented to one eye for a variable adaptation period. Then a competing stimulus was presented to the other eye in flash suppression. The novel manipulation was the quick removal of the flashed stimulus, after only hundreds of milliseconds. Rather than returning to conscious perception of the only remaining stimulus, the brain seemed to continue to select the eye channel representing now only a black homogeneous background.

While this effect can be interpreted in a framework of binocular rivalry processes, there are other demonstrations of visual targets disappearing from conscious perception, such as motion-induced blindness (Y. S. Bonneh, Cooperman, \& Sagi, 2001), filling-in (see Pessoa \& de Weerd, 2003), and Troxler's fading (Troxler, 1804). Troxler's fading occurs when one stares at a fixation point for a prolonged period of time: visual percepts in the periphery will begin to 'disappear' or fade, possibly due to fatiguing edge detectors (e.g. Ramachandran \& Gregory, 1991). Related or not, similar 'fading' effects can be observed when flashing transients near peripheral stimuli (transient-induced fading: Kanai \& Kamitani, 2003), or when abruptly changing peripheral stimulus contrast (contrast-decrement-induced fading: May, Tsiappoutas, \& Flanagan, 2003). Under the right conditions, even entire complex scenes can fade from conscious perception (Simons, et al., 2006). It appears that all these fadings effects immediately end when a (micro-)saccade occurs, which is something that we have observed in the DRE phenomenon. Since our stimuli were also peripheral, this raises the question whether such fading mechanisms underlie, fully or in part, the DRE. This leads also to the more general question: how often our visual stimulus elements would disappear from conscious view spontaneously and for what durations?

To further characterize the DRE and understand its underlying mechanisms we evaluated in the same group of participants the pattern of effects of manipulation of one independent variable (eccentricity of competing stimuli) on four phenomena: 1) binocular rivalry dominance period duration, 2) disrupted rivalry effect duration, 3) presence and durations of spontaneous fading with one stimulus presented to one eye, 4) presence and durations of spontaneous fading with the same stimulus presented to both eyes. This design allowed us to compare absolute durations and disappearance frequency between conditions. It is perhaps conceivable that condition 3) involved rivalry between black background and one stimulus, in which case it might elicit the 
same frequency and durations of disappearing stimulus elements. On the other hand, if fading or filling-in were the only mechanisms at work, there could be a resemblance of effects between the disrupted rivalry and fading conditions 3) and 4). By manipulating the effects for all conditions with one identical additional factor, eccentricity of stimulus elements, we could moreover compare qualitatively, and quantitatively after normalization, the pattern of effects of eccentricity on these measures.

\section{Materials and Methods}

\section{Participants}

Since the purposes of the current experiment involved the correlation of normalized effects across participants, a large subject sample was required. 27 participants volunteered for this study to partially fulfill course credit requirements. 20 of these were naive to the goals and contents of the current experiment, all were new to binocular rivalry and untrained in maintaining fixation. All had normal or corrected-to-normal vision and provided written informed consent. The experiment was approved by the local ethics committee.

\section{Stimuli, Tasks and Design}

Stimuli included the previously described 'blank' images, as well as 'star' and 'triangle' images of always one visual element in one peripheral location in one of the four visual quadrants. There were moreover three possible locations in terms of eccentricity. These were equidistant, with the middle eccentricity corresponding to previous experiments. There were thus 2 (star vs triangle) x 4 (visual field quadrant) x 3 (eccentricity) stimulus images.

The task for subjects was always the same: 'indicate by pressing and holding keyboard button [NUM5] when the initial stimulus disappears from view - release the button when you perceive it again'. There were however four different conditions in the experiment. In the binocular rivalry condition (BR), there was an adaptation phase of 5000 seconds in which one stimulus was presented to one eye, after which a competing stimulus was presented in addition to the second eye (becoming dominant $=$ flash suppression [Wolfe 1984]). Subjects indicated the return of the adapted stimulus by releasing the button. In the disrupted rivalry condition (DR), the flashed second stimulus was removed after a rivalry time of $300 \mathrm{~ms}$. Subjects indicated return of the adapted stimulus by releasing the button. In a third condition there was never a rivalry phase: the initial image was continually presented to the one eye, with a blank image presented to the other eye for the duration of the trial. IF the stimulus were to disappear in this condition, it might be because of binocular rivalry between the blank image and the salient stimulus, or because of Troxler's fading effect. For convenience, we refer to this condition as F1 (fading with 1 stimulated eye). In a fourth condition, both eyes were presented with the same (thus non-competing) stimulus element throughout the trial duration. Any stimulus disappearance in this condition would therefore not be 
attributable to binocular rivalry processes. We refer to this condition as F2 (fading with 2 eyes). Each trial always lasted 10 seconds.

Each participant was measured in two runs in one session. There were 4 (BR, $\mathrm{DR}, \mathrm{F} 1, \mathrm{~F} 2) \times 4$ (visual field quadrant) $\times 3$ (stimulus eccentricity) $\times 2$ (adapted stimulus) x 2 (eye/monitor with adapted stimulus) conditions (except for F2 condition), each tested twice, leading to a total of 336 trials per session/participant.

\section{Analysis}

Trials in which participants did not follow instructions or had unrealistic RTs $(<200 \mathrm{~ms})$ were excluded prior to analysis; outliers were removed if more than 2.5 subject- and main condition-specific standard deviations above the mean.

The proportion of trials in which effects (nucleations for BR, DRE for DR, disappearance for F1, F2) occurred per subject were evaluated in a repeated-measures ANOVA (RM-ANOVA, Huynh-Feldt corrected) to evaluate whether some effects were less common than others.

The distributions of effect durations for each main condition (BR, DR, F1, F2) were displayed in (normalized) binned histograms. They suggested that average values of effect durations over participants would be inadequate representations of the data for the purpose of comparing durations between main conditions, so median values were extracted per participant per main condition for statistical analysis. Non-parametric Signed-Rank tests (bonferroni-corrected) compared the median effect durations between conditions.

As this analysis suggested that the effects measured by the four main conditions were potentially subserved by different neuronal mechanisms, the effects of eccentricity were evaluated separately for each main condition (BR, DR, F1, F2) using RM-ANOVAs and follow-up tests as indicated in the Results section. But to nevertheless compare qualitatively and quantitatively the pattern of effects of eccentricity between these conditions, the data in each condition were normalized around individual means per condition. This brought the measures in the same 'space', allowing more direct comparison. The effects were correlated using Pearson's correlation, as reported in the Results section.

\section{Results}

For conditions BR (binocular rivalry) and DR (disrupted rivalry) there was always an effect duration by default, as the rivalry phase by itself required the button press. In the 'fading' conditions however (F1, F2), it was not necessarily the case that the stimulus element would disappear from conscious perception on each given trial. In fact, this event could be rather rare, given that a very clear stimulus was presented in both conditions. Nevertheless, disappearance could occur, by the various mechanisms outlined in the Introduction. 


\section{proportion of trials with stimulus element disappearance}

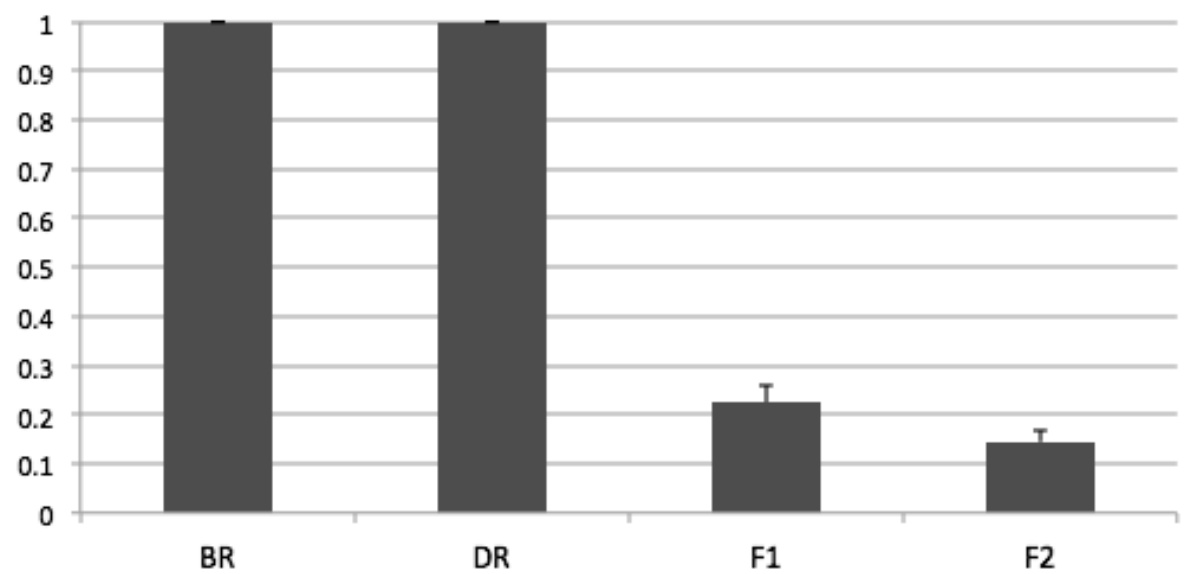

Figure 1: proportion of trials with disappearing stimulus elements

The proportion of trials in which participants reported not seeing the 'adapted' stimulus element at least once, shown separately per main condition $(\mathrm{BR}=$ binocular rivalry; $\mathrm{DR}=$ disrupted rivalry; F1 = 'fading' 1 : for the duration of the trial (10 seconds) one eye was presented with a stimulus element and the other eye was not; F2 = fading 2: both eyes presented with same stimulus element for the duration of a trial). For conditions BR and DR, since a different stimulus flash-suppressed the adapted stimulus at least briefly, nonperception of the adapted stimulus was by design reported on each trial. Most important are the findings that participants continued to see the stimulus element uninterrupted for the full duration of trials most of the time in conditions F1 and F2, although significantly more often in F2. Within-subject error bars (Cousineau, 2005)

\section{Effects of condition on prevalence and duration of effects}

Figure 1 shows the proportion of trials per condition in which disappearance occurred, averaged over participants. The number of trials in which disappearance occurred was clearly dependent on condition $(\mathrm{F}=621.9 ; \mathrm{P}<0.001)$. Of interest was the fact that the proportion of trials with disappearing stimulus elements was significantly different between F1 and DR, but also between F1 (disappeared stimulus report on $22.4 \%$ of trials) and F2 $(14.4 \%$ of trials) $(\mathrm{Ps}<0.001)$. In condition F2, since both eyes were presented with the same image, there could be no rivalry and disappearance effects should be due to mechanisms underlying for example Troxler's fading. In condition F1, it seems possible that the additional proportion of trials with effects is due to rivalry between the stimulus element to one eye and the black background to the other eye. But this is not straightforward, as in F1 there was also only 'half the stimulation' presented to the visual system as a whole. This decreased total sensory input might also affect the likelihood of fading. 

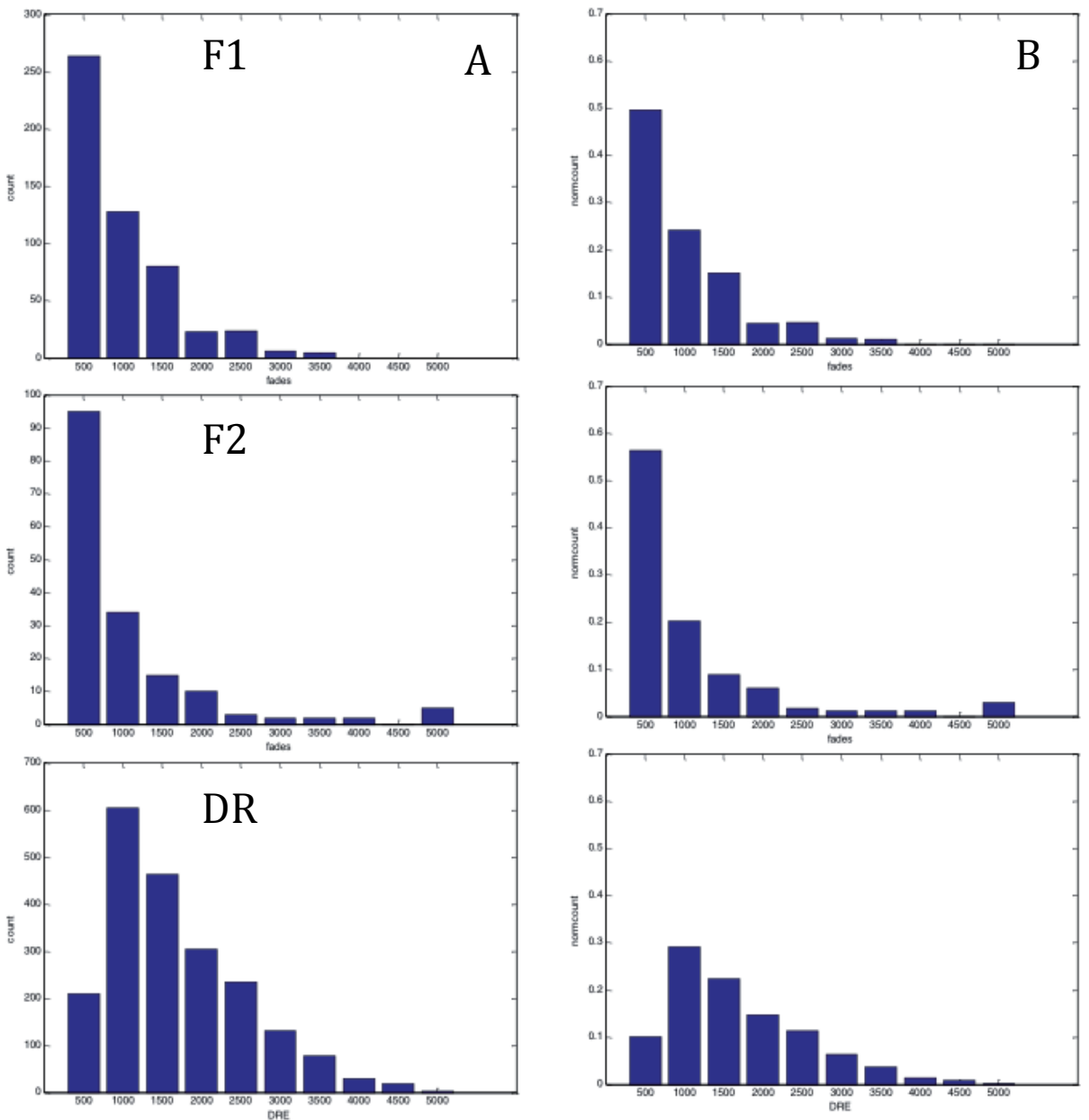

Figure 2: distributions of stimulus element disappearance durations A. Shown separately for condition F1 (top), condition F2 (middle), and condition DR (bottom) are binned (horizontal axis) counts (vertical axis) of the durations of perceived stimulus element disappearance (always with stimulus element actually present). These distributions show that for both F1 and F2 conditions, the norm for effect durations was clearly a disappearance duration of only hundreds of milliseconds. Given the strong skewing to the right, and the maximal duration of 10 seconds for F1/F2, and 4.7 seconds for DR, mean durations would constitute inadequate reflections of the data distribution for the purposes of comparing conditions directly in terms of effect durations. B. Same as in Figure 2A, but counts were normalized and axes identical, to offer more direct comparison of distributions.

To further investigate the potential mechanisms underlying the effects in conditions BR, DR, F1, and F2, we plotted the distributions of the effect durations in trials that showed an effect (i.e. trials of F1, F2 in which no disappearance occurred were not included). If on a given trial of F1 or F2 there was more than 1 instance of reported disappearance, the durations of these periods were averaged into one 
incorporated value. Figure 2A shows the distributions over all subjects per main condition. Figure 2B shows normalized distributions with identical axes. It is clear that, if there was disappearance of perceived stimulus elements on a given trial in F1 or F2, the duration of disappearance was much shorter than in the case of DR.

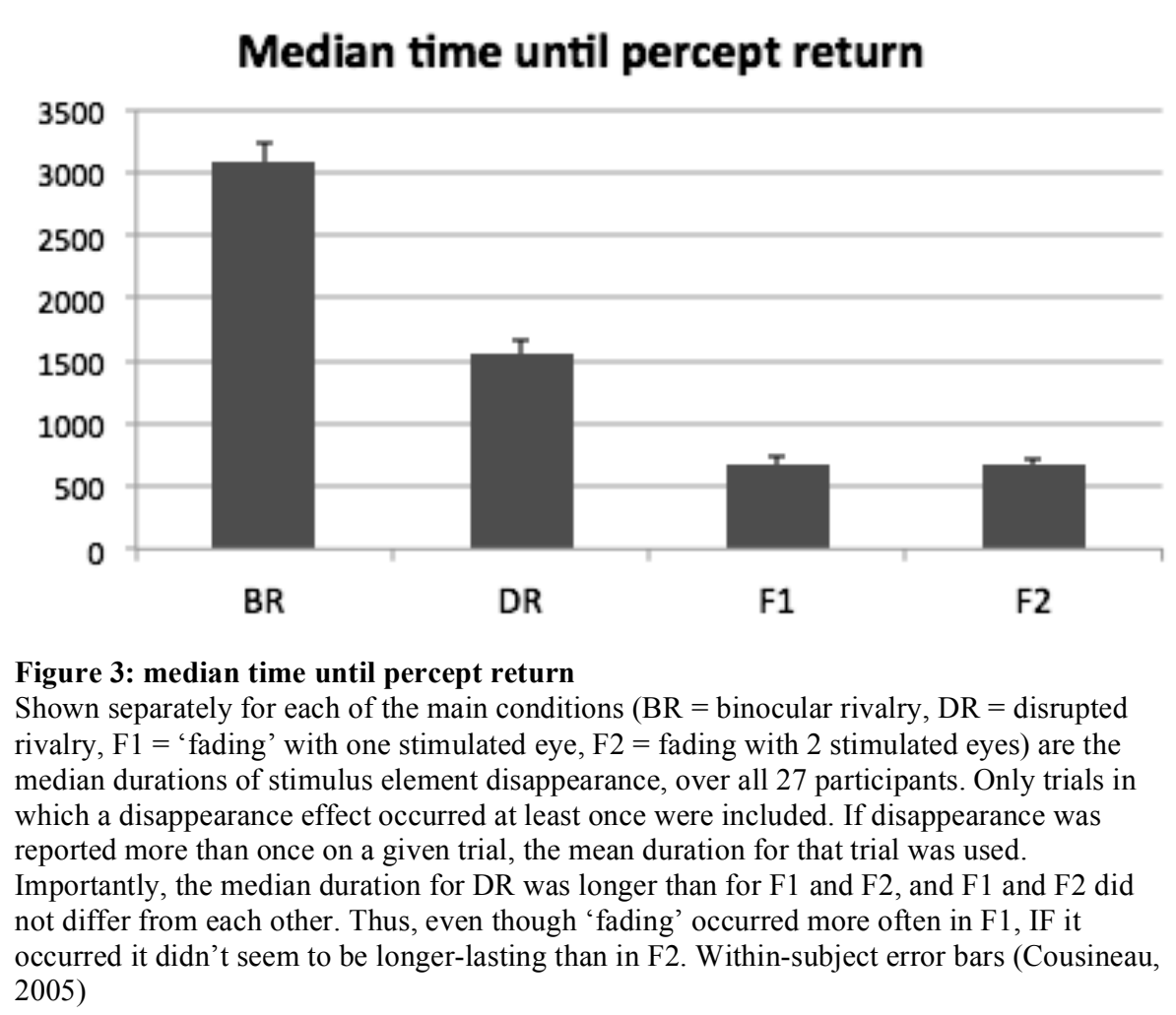

To test this statistically, we extracted median values for the effect durations of conditions BR, DR, F1, and F2, including only trials of F1, F2 in which effects actually occurred. (The fact that there was a range of 10 seconds for potential fading effects $(10$ second trials) but less than 5 seconds for DRE durations (first 5 seconds were adaptation), combined with the shapes of the distributions in Figure 2, suggested that mean effect durations would not accurately reflect the data for the purposes of comparing these conditions.) Figure 3 displays the average median effect durations over participants. Related-samples Friedman's 2-way ANOVA by Ranks showed that there was a main effect of Cond (BR, DR, F1, F2). Wilcoxon signed-rank tests showed that medians in all conditions differed significantly from each other $(\mathrm{Ps}<0.001)$ except for $\mathrm{F} 1$ and $\mathrm{F} 2(\mathrm{P}=0.819)$. 

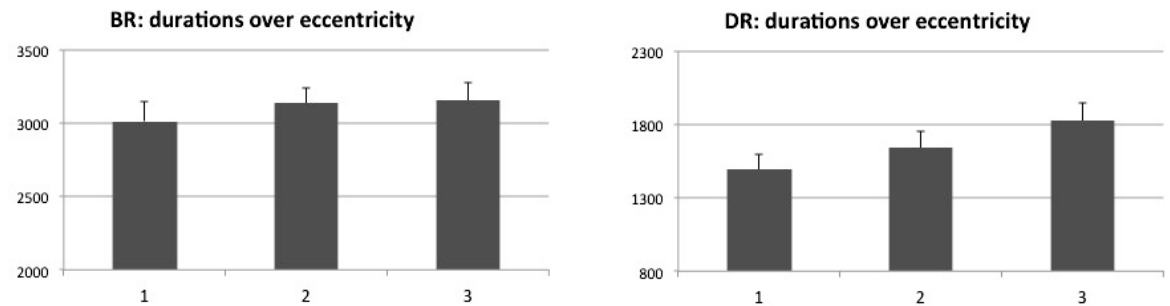

F1: durations over eccentricity

F2: durations over eccentricity
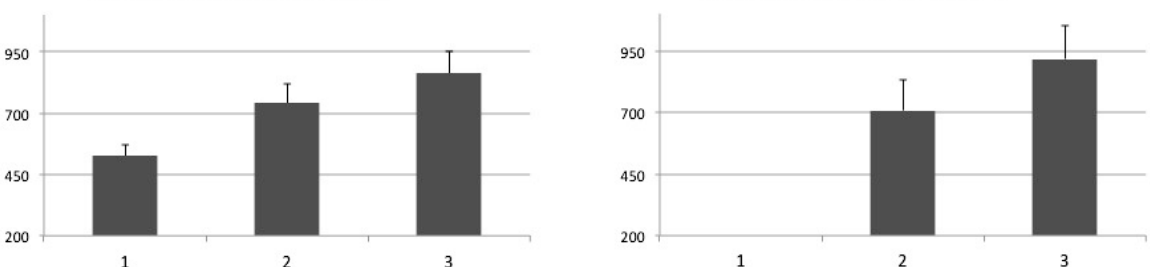

Figure 4: The effect of stimulus eccentricity on durations of stimulus element disappearance

Shown separately per main condition are the mean durations of stimulus element disappearance per level of eccentricity (horizontal axis: $1=$ stimulus element closest to fixation, 2 = 'standard' eccentricity used in other chapters, middle distance to fixation, $3=$ stimulus element furthest from fixation). For F2, there were too few trials per participant for eccentricity 1 to obtain a reliable estimate. Overall, it appears that there is a rising trend in effect durations with increasing eccentricity.

\section{The effect of eccentricity}

Figure 4 shows the mean effect durations of BR, DR, F1 and F2 over the three levels of eccentricity. In condition F2, for the lowest eccentricity (stimulus element closest to fixation), there were only 2 participants with more than 2 trials in which disappearance was reported, so this level was omitted. For DR, F1 and F2 there is on the whole a clear increase in effect durations with increasing eccentricity. For BR, this effect seems slightly less clear and less linear. RM-ANOVAs per condition with factor eccentricity (Ecc) confirmed a significant effect of Ecc for DR $(\mathrm{F}=16.2 ; \mathrm{P}<0.001)$ and $\mathrm{F} 1(\mathrm{~F}=15.1$; $\mathrm{P}<0.001)$, but not for $\mathrm{BR}(\mathrm{P}=0.221$, uncorrected). For $\mathrm{F} 2$, there was also no significant effect, but this should not be taken very strongly considering the number of trials involved (on average 2.7 trials per participant per level, and that is still leaving out the participants without any trials with effects).

Considering the strong differences in absolute effect durations, we normalized the effects over levels of eccentricity per condition (BR, DR, F1, F2) per participant. Figure 5 shows the averages of these normalized results plotted together. Overall, there seems to be a rising trend, indicating that with increased eccentricity dominance durations of the flashed stimulus (BR), the duration of DRE (DR), and the duration of spontaneous 'fading' (F1) increase. Since these data were standardized, a RM-ANOVA was performed with factors Cond (BR, DR, F1) x Ecc (eccentricity; 3 levels). There was a main effect of Ecc $(\mathrm{F}=10.5 ; \mathrm{P}<0.01)$, but no interaction with Cond. Also, the 
patterns of effects over Ecc (i.e. differences between normalized scores for different levels of Ecc) did not correlate significantly between DR and BR, but also not between DR and F1 or F2.

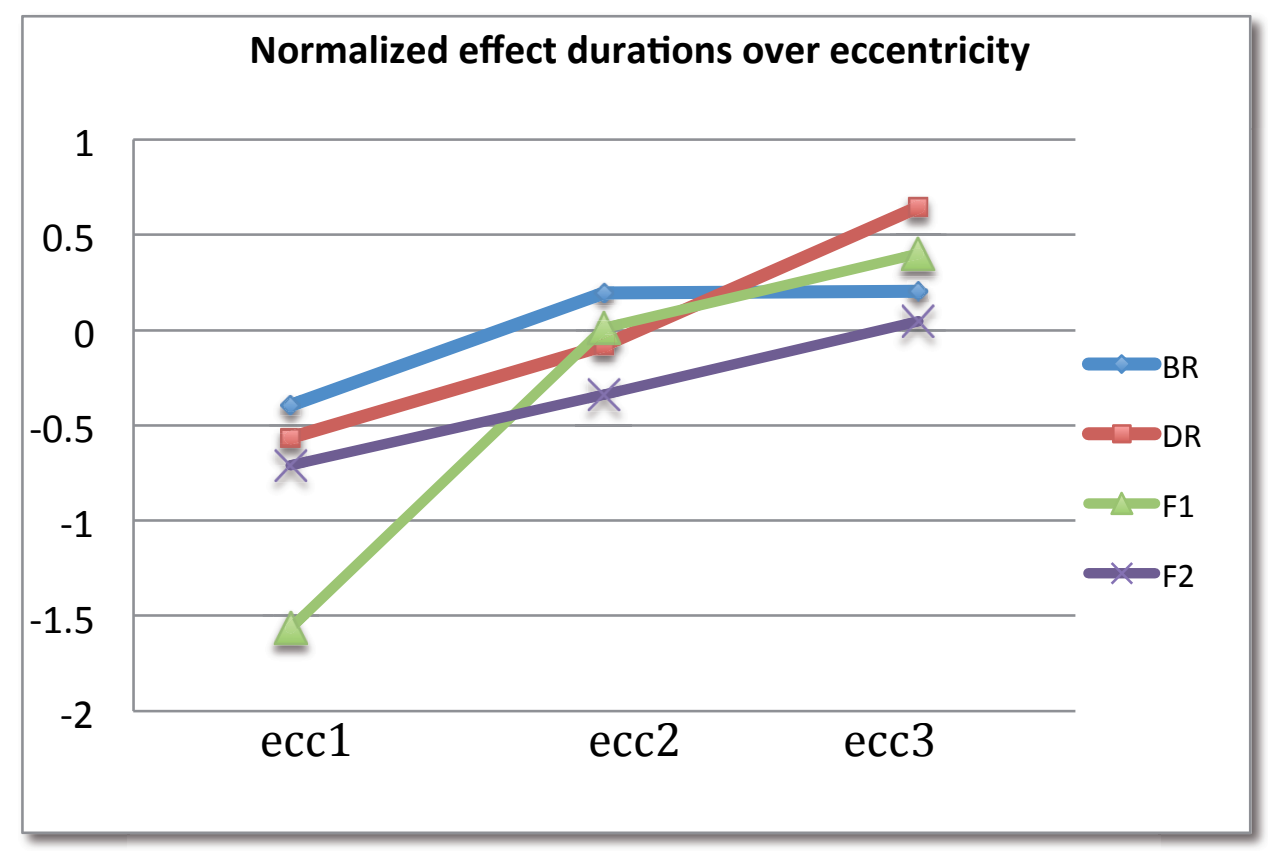

Figure 5: normalized time to percept return over eccentricity Shown separately for the four main conditions are the effect durations, including only trials in which effects occurred, averaged over participants. It appears the effect of eccentricity is largely the same for all conditions, and indeed there was no significant interaction between stimulus eccentricity and condition on the normalized effect durations.

\section{Discussion}

One important question in appreciating and interpreting the DRE established in chapter I.1, concerned the frequency and duration of disappearing stimulus elements in the same setup but without the 'disrupted rivalry protocol'. In short: do the elements also disappear spontaneously, and if so for how long? We here tested this question by presenting one stimulus element for up to ten seconds, to one eye and also to both eyes. It was clear that the spontaneous disappearance of the elements was not common, but that it did happen occasionally. It happened more often when one eye was stimulated, and the other eye was presented only with homogenous black background in the corresponding location, as compared to when both eyes were presented with the same stimulus element. However, when evaluating the median durations of the disappearance, this duration was not different between the conditions with one or both eyes stimulated (F1, F2) on trials with reported stimulus disappearance. Moreover, both 
durations were shorter than in the disrupted rivalry trials. One candidate mechanism contributing to DRE may be fading, as in Troxler's fading (Troxler, 1804). As conditions F2 and F1 measured possible Troxler fading effects (or other mechanisms in which stimulus elements could disappear spontaneously, see Introduction), it appears DRE cannot be fully explained by such mechanisms. We cannot yet determine whether DRE is (related to) a form of transient-induced fading (Kanai \& Kamitani, 2003), although experiments in chapters I.1, I.3, I.4 support at least a contribution of binocular rivalry mechanisms.

Here we also measured the effect of one independent variable (eccentricity of competing stimuli) on multiple measures in the same subjects (dominance duration in binocular rivalry (BR) after flash suppression, DRE after flash suppression, 'fading' with 1 stimulus presented to one eye, and fading with 1 stimulus presented to both eyes). For all conditions, increased eccentricity led to increased effect durations, although this did not reach significance for BR. The pattern of effects did not correlate between DR and any other condition, over participants. This may suggest that, while fading and binocular rivalry can contribute to the DRE, there is no one-to-one correspondence between them, although the lack of correlation is a null result that is difficult to credit.

For the moment, we should consider that DRE may be related to several mechanisms. Speculatively, for example the double-inhibition in the binocular rivalry framework may form the basis of DRE, but strengthened by similar mechanisms underlying Troxler's fading or transient-induced fading, or filling-in. The data from experiment 1 suggest that certainly the mutual inhibition (binocular rivalry) mechanism may contribute to DRE, but that the full story may be more complex. 



\title{
Characterizing the Disrupted Rivalry
}

\author{
Effect (II): decreased DRE duration by
}

\author{
gradual stimulus onset and offset
}

Related publication(s):

De Graaf, TA, van Ee, R*, Croonenberg, D, Goebel, R, Sack, AT (in preparation). The disrupted rivalry effect: the role of interocular stimulus offset

* Raymond van Ee

1) Department of Physics of Man, Helmholtz Institute, Utrecht University, Utrecht 3584 $\mathrm{CH}$, The Netherlands

2) Department of Brain, Body, Behaviour, Philips Research Laboratories, Eindhoven 5656 AE, The Netherlands

3) Department of Experimental Psychology, Leuven University, Leuven B-3000, Leuven, Belgium 


\begin{abstract}
Binocular rivalry involves the stochastic fluctuation in conscious perception between two competing representations, one of an image presented to the left eye, the other of an image presented to the right eye. Previously we introduced a potentially new phenomenon referred to as the disrupted rivalry effect (DRE). This effect was the prolonged failure to perceive a salient visual stimulus following abrupt onset and offset of a rivaling stimulus in quick succession. We interpreted this effect by means of a 'double-inhibition' account of mutually inhibiting eye-channel representations. This account makes several testable predictions. As the double-inhibition model postulates that abrupt stimulus offset in one eyechannel results in an 'inhibitory boost' of the competing channel, one specific prediction is that more gradual flashed stimulus on/offsets should decrease the effect duration. We here tested and confirmed this prediction.
\end{abstract}




\section{Introduction}

Binocular rivalry occurs when two incongruent images are presented to both eyes. The representations of these two possible percepts compete for access to visual awareness, with the result that conscious perception fluctuates over time. An ongoing debate focuses on the areas in the brain (Leopold \& Logothetis, 1996; Tong, et al., 2006) and stage of visual processing (Blake \& Logothetis, 2002; Freeman, et al., 2005) in which this competition occurs. While today consensus suggests that both low- and higher-level stages in the visual hierarchy are involved, it remains unclear to what extent and in which situations competition is focused on (for example) eye-channels (Blake, 1989) or higher-order representations (Logothetis, et al., 1996), although this may depend on stimuli and tasks (Y. Bonneh, et al., 2001)

Irrespective of the locus of competition, a relatively simple model described in chapters I.1-2 can account for many of the observations underlying these binocular rivalry phenomena. In this model (although simplified, see (Noest, van Ee, Nijs, \& van Wezel, 2007) for a recent example of a more mathematically rigorous implementation), the representation (or eye-channel) of one image inhibits the representation (or eyechannel) of the other image, and vice versa.

Recently, we introduced a phenomenon that may be related to this mechanism, referred to as the 'disrupted rivalry effect' (DRE). DRE involves the lasting (up to several seconds) suppression of a salient stimulus without any 'real' competition. It was elicited by first adapting an eye to a peripheral visual stimulus (adaptation phase), then adding a fresh competing stimulus to the other eye (rivalry phase) ensuring its immediate perceptual dominance (= flash suppression, see Wolfe, 1984). The novel manipulation was to immediately (after only hundreds of milliseconds) remove this flashed stimulus, reinstating the homogenous black background. Intuitively, one might expect that conscious perception would simply revert to the remaining, salient, previously dominant stimulus. Yet, participants reported seeing 'nothing' for quite some time, in the order of seconds.

We speculated that this DRE demonstrated the propensity of the visual system to appreciate visual change, thus including stimulus offsets, rather than constant visual stimulation (Macknik \& Livingstone, 1998; Macknik, et al., 2000; Roland, 2010). We proposed that the stimulus offset, quickly following the abrupt stimulus onset, constituted a second inhibition-boost in the mutual-inhibition framework. The eyechannel corresponding to the remaining stimulus (although salient) might be inhibited so strongly by this 'double-inhibition' that it did not re-attain dominance for a prolonged period of time. This interpretation would suggest to us that the abruptness of the onset of the flashed stimulus, as well as its offset, is crucial. Presumably, the inhibition signal is stronger with a square-wave (instantaneous) onset/offset, as compared to a ramped (gradual) onset/offset. In the current study, we test this prediction by repeating the experiments with square-wave (0 ms ramp), fast-gradual (150 ms ramp), and slowgradual (300 ms ramp) flashed stimulus on/offsets. 


\section{Materials and Methods}

\section{Participants}

Ten participants volunteered for this experiment. Nine were naive to the goals and content of the experiment, all were absolutely inexperienced in binocular rivalry and generally untrained in fixation. They had normal or corrected-to-normal vision and provided written informed consent. They were compensated with monetary coupons. The experiment was approved by the local ethics committee.

\section{Stimuli, Task, and Design}

Stimuli and experimental setup were identical to experiments 2-4 described in chapter I.1. The task on each trial was to 'press the keyboard button when the initially presented image is again fully visible.' The initially presented image was either star or triangle, selected at random, presented by itself to one eye for an adaptation phase (always lasting $4000 \mathrm{~ms}$ ). A competing stimulus was then presented to the other eye during the rivalry phase. There were two main conditions in the experiment: binocular rivalry (BR) trials contained only the adaptation phase and then involved rivalry until the end of trial, while disrupted rivalry (DR) trials also included rivalry phase (always $300 \mathrm{~ms}$ ) and subsequent potential DRE phase. The baseline condition (CTR, see chapter I.1) was omitted, as previous experiments had established the consistent existence of DRE.

The standard setup in previous experiments was a square-wave ( $0 \mathrm{~ms}$ ramp $)$ onset and offset of this rivalry stimulus. We here implemented gradual on-/offsets to create the following conditions: 1) square-wave onset/offset, 2) ramped onset/offset, 3) ramped onset only (and square-wave offset), 4) ramped offset only (and square-wave onset), 5) ramped onset/offset. Moreover, we implemented two different rates of onset/offset. For fast-ramp trials, the process of stimulus presentation from naught to fully luminant (=equal luminance to square-wave stimulus) took $150 \mathrm{~ms}$. This gradual process was centered around the onset of the stimulus in square-wave trials. Thus, in relation to the onset of the stimulus in the square-wave trials, half $(75 \mathrm{~ms})$ of the ramping in fast-ramp trials occurred before and half $(75 \mathrm{~ms})$ occurred after this onset. The same for offset fast-ramp trials. For slow ramping, the ramping process took 300 $\mathrm{ms}$, again centered around the onset or offset of the square-wave stimulus in none-ramp trials. Thus, for example in the trials with slow-ramp onset and slow-ramp offset, the second stimulus would immediately begin to disappear as soon as it was fully luminant (i.e. in non-ramp trials the full stimulus duration was $300 \mathrm{~ms}$, second half of slow-ramp onset ( $=150 \mathrm{~ms})$ and first half of slow-ramp offset ( $=150 \mathrm{~ms}$ ) add up to $300 \mathrm{~ms}$ ).

With no-ramp, both-ramp (fast and slow), onset-ramp (fast and slow), offsetramp (fast and slow), there were a total of 7 conditions for DR trials. BR trials were divided into trials with no-ramp, trials with fast-onset ramp and slow-onset ramp, thus a total of 3 conditions. Each of the 10 condition cells was measured 32 times per subject, leading to a total of 320 trials measured in two runs. Participants were given self-paced 
breaks during the measurement with the opportunity to move around, turn on the light, and relax their eyes.

\section{Analysis}

Trials in which instructions were not followed properly (e.g. responses at wrong moments in trial; 144 trials $=4.5 \%$ of total) or RTs were unrealistically short (i.e. less than $200 \mathrm{~ms}$; 51 trials: $1.7 \%$ of total) were excluded prior to analysis. Outliers were removed and determined by the criterion that RTs could not exceed 2.5 participantspecific and BR-/DR-specific standard deviations above the mean. One participant was removed because his/her responses over all conditions (i.e. also BR) were over 2 standard deviations below the group mean over all subjects, leaving 9 participants. Repeated-measures ANOVAs (RM-ANOVAs, Huynh-Feldt corrected) were used to analyze the data. Due to the specific conditions in the experiment, a full-model ANOVA including all conditions was not possible. We thus determined a priori the following RM-ANOVAs of interest.

The first RM-ANOVA included factors Cond (BR, DR) and Onset-Ramp (Noramp [No-R], Onset-Ramp [On-R]). Second, for DR specifically a full-model RMANOVA evaluated factor Ramp (No-R, On-R, Off-R, On/Off-R), allowing a first evaluation of whether or not gradual stimulus presentation had any effects as compared to the original paradigm (i.e. No-R), followed by planned comparisons of On-R, Off-R and On/Off-R, all versus No-R by one-tailed paired-samples t-tests. Third, to allow comparisons including the factor RampSpeed, a 3x2 RM-ANOVA was run including factors Ramp (On-R, Off-R, On/Off-R) and RampSpeed (fast, slow). But since On/Off$\mathrm{R}$ conditions were strictly not independent from the single-ramped conditions, a $2 \times 2$ RM-ANOVA was evaluated including factors Ramp (On-R, Off-R) and RampSpeed (fast, slow). Any follow-up analyses as presented in the Results section.

\section{Results}

The RM-ANOVA with factors Cond (BR, DR) x Onset-Ramp (No-R, On-R) revealed a main effect of Cond $(\mathrm{F}=16.2 ; \mathrm{P}<0.01)$ and Onset-Ramp $(\mathrm{F}=6.0 ; \mathrm{P}<0.05)$, but no interaction $(\mathrm{P}=0.517)$, suggesting that the effect of gradually presenting the competing second stimulus is similar in a situation of binocular rivalry and in a situation of disrupted rivalry. As shown in Figure 1, the effect in both cases is a decrease of response time (RT) indicating dominance duration (for BR) or duration of the DRE (for DR).

The RM-ANOVA for disrupted rivalry trials with factor Ramp (No-R, On/Off$\mathrm{R}$, On-R, Off-R) confirmed that there was an effect of Ramp $(\mathrm{F}=10.8 ; \mathrm{P}<0.01)$, and follow-up tests (one-tailed, bonferroni-corrected for 3 planned comparisons) showed that all three gradual stimulus presentation/removal conditions significantly decreased the duration of DRE as compared to No-R (On/Off-R: $\mathrm{t}(8)=3.7 ; \mathrm{P}<0.05$, On-R: $\mathrm{t}(8)=2.8$; $\mathrm{P}<0.05$, Off-R: $\mathrm{t}(8)=3.3 ; \mathrm{P}<0.05)$. Thus, gradual onset of the rivaling stimulus, but also gradual offset of the rivaling stimulus, significantly decreased DRE duration. The durations per level of Ramp are shown in Figure 2. 


\section{Gradual Onset of Rivalling Stimulus}

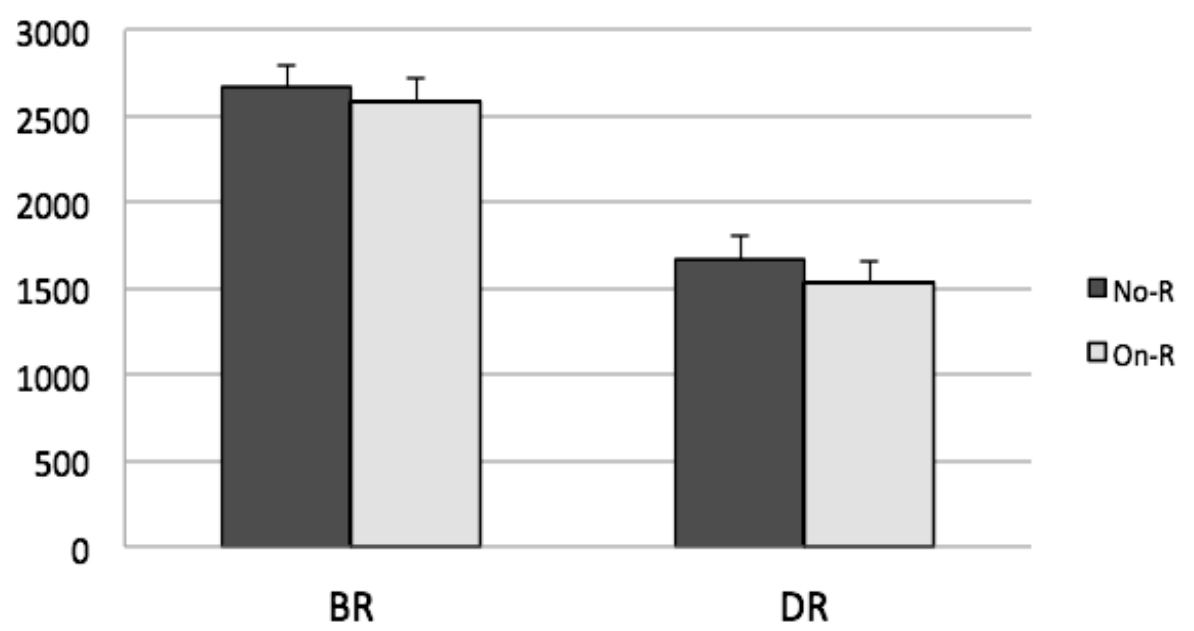

Figure 1: Gradual Onset of Rivaling Stimulus

Shown separately for condition BR (binocular rivalry) and condition DR (disrupted rivalry) are the reaction times (for BR: time to first perceptual switch back to the originally adapted stimulus, for DR: time to return to awareness of the only remaining, originally adapted, stimulus) for condition No-R (no ramp: abrupt onset of rivalling stimulus) and On-R (gradual onset of rivalling stimulus). The speed of 'ramping' (i.e. how gradual it was) was collapsed here. Within-subject error bars (Cousineau, 2005).

The RM-ANOVA for factors Ramp (On/Off-R, On-R, Off-R) and RampSpeed (fast, slow) revealed a main effect of Ramp $(\mathrm{F}=8.9 ; \mathrm{P}<0.01)$, and of RampSpeed $(\mathrm{F}=14.1 ; \mathrm{P}<0.01)$, but also an interaction between the two $(\mathrm{F}=4.2 ; \mathrm{P}<0.05)$. Thus, there is not only an effect of the speed of rivaling stimulus onset and offset, but this effect depends on whether onset or offset (or both) is graded. This analysis might not be wholly adequate, however, since the effect in On-/Off-R trials may comprise the potential effects in On-R and Off-R trials. Thus, a 2x2 RM-ANOVA containing factors Ramp (On-R, Off-R) and RampSpeed (fast, slow) was run to confirm this potential interaction. Again, there was a significant interaction between Ramp and RampSpeed $(\mathrm{F}=7.4 ; \mathrm{P}<0.05)$.

To further evaluate these interactions, paired-samples t-tests (1-tailed, bonferroni-corrected) compared the DRE durations between fast and slow ramping, separately for each level of Ramp. This analysis suggested significantly decreased DRE for the more gradual rivaling stimulus On-/Offset (slow ramp DRE $<$ fast ramp DRE $t(8)=2.9 ; \mathrm{P}<0.05$ ), no significant difference for the speed of ramped Onset, and again a significantly sharper decrease in DRE duration for more graded rivalry stimulus Offset (slow ramp DRE $<$ fast ramp DRE $-\mathrm{t}(8)=3.2 ; \mathrm{P}<0.05$ ). In Figure 3 we show the DRE durations for these various conditions, and provide for the sake of comparison the DRE duration in the 'original' DR paradigm with abrupt stimulus On-/Offset. 


\section{DRE durations with gradual on-/offsets}

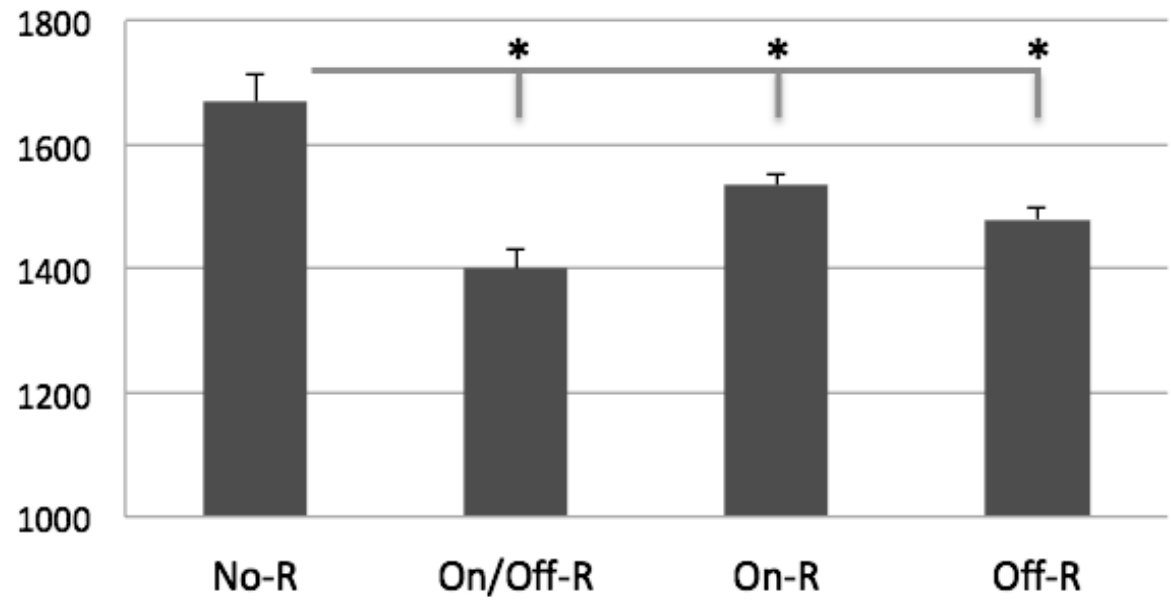

Figure 2: The effect of gradual rivalling stimulus on-/offsets on DRE duration Shown for the same participants are the DRE durations (RT in disrupted rivalry trials), separately for the 'original' abrupt, or No-R (no-ramp), condition, and the conditions On/Off$\mathrm{R}$ (both rivalling stimulus onset and offset are gradual - collapsed over ramp speed), On- $\mathrm{R}$ (only the onset is gradual, collapsed over ramp speed), Off-R (only the offset is gradual, collapsed over ramp speed). As indicated by asterisks, DRE in each of the gradual on-/offset conditions was significantly shorter-lasting than the no-ramp (No-R) condition. Withinsubject error bars (Cousineau, 2005).

\section{Discussion}

From previous work establishing the disrupted rivalry effect (DRE), we derived several predictions. A double-inhibition account explained why a nearly complete lack of signal (homogeneous black background) might successfully dominate and suppress a salient peripheral stimulus representation in the competing eye channel. In a simple mutualinhibition scheme, a fresh stimulus would strongly inhibit an already somewhat adapted image representation. This was previously known as flash suppression (Wolfe, 1984). But a second boost of inhibition was suggested to result from the stimulus offset. We proposed that the quick succession of these two inhibition boosts suppressed the eye channel strongly. The lack of any visual change in the suppressed eye channel ensured that the recovery of the suppressed eye channel was slow.

This interpretation led to the hypothesis that the abruptness of the visual change was crucial for the DRE. We thus tested here the effect of ramping (quickly or more slowly) the onset, offset, or onset and offset of the flashed stimulus. As hypothesized, this ramping decreased the duration of DRE. This decrease was, however, surprisingly modest. Based on our framework, we more or less expected an abolishment 
DRE durations: ramp speed

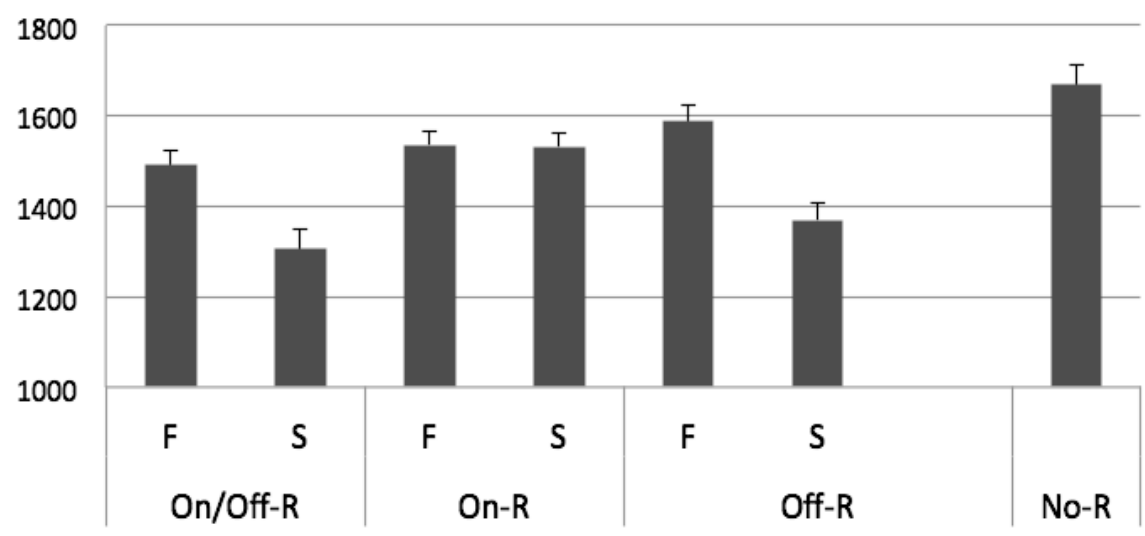

Figure 3: DRE durations for gradual on-/offsets at two ramp speeds

Shown are the DRE durations (RTs in disrupted rivalry trials), for gradual onset/offset trials (On/Off-R), gradual onset only trials (On-R), and gradual offset only trials (Off-R), as well as original abrupt on-/offsets trials (No-R) for comparison. For the trials with gradual (ramped) on- and/or offset, there were two conditions with either fast (F), or slow (S) ramping. Withinsubject error bars (Cousineau, 2005).

of DRE or strong decrease of its duration. This modest decrease may indicate that the mutual inhibition model describes only partly the mechanisms underlying DRE. It is possible, of course, that even slower ramping would further decrease the effect. But if the rivalry phase is to remain in the range of hundreds of milliseconds, there is a fundamental limit to how long the ramping process can take. Moreover, the effect of ramping was, aside from modest, not unequivocal. It was relatively strong for stimulus offsets, and scaled with ramping speed. This is in accordance with our previous interpretation. But for stimulus onsets, this was not the case. Although there was a decrease of DRE with ramped onset, this was small and did not scale with ramping speed. Thus, although these results partly confirmed our hypotheses, they also suggest that the DRE may be subserved by multiple, or more complex mechanisms.

There is an important methodological caveat in this experiment that could affect these and other results. As specified in the Methods section, the 'ramping' or gradual on- and/or offsets of rivaling stimuli were centered around the time point of square-wave stimulus on- and/or offset. The response times were therefore related to this same time point. However, for example with ramped stimulus offset, the image would not be fully removed from view for another 75 (fast-ramp) or 150 (slow-ramp) milliseconds. If we assume that the correct time point to relate the start of DRE to is the complete absence of the rivaling stimulus, this would decrease the DRE by a further $150 \mathrm{~ms}$, strengthening our conclusions. Since it seems unclear which is the more reasonable time point to start measuring DRE from, we selected the (in our view 
conservative) middle-point in the ramping process. For ramped stimulus onsets, the situation is reversed. Again we conceived of the rivalry-onset as the midpoint of the onset-ramping process. However, it could be argued that the correct starting point is earlier, more towards the first 'onset' of the stimulus element that is slowly increasing in luminance. The 'true' onset of rivalry depends on when the participant first becomes aware of the 'flashed' rivalry stimulus. For the binocular rivalry condition (BR), this directly affects the response time, as here the response time reflects the dominance duration of the 'flashed' rivalry stimulus. For the disrupted rivalry condition, however, even though DRE is related to rivalry stimulus offset, this may also have an effect. In previous experiments we showed that rivalry phase duration negatively relates to the duration of the DRE. Thus, if the rivalry onset is in actuality earlier, the rivalry phase is essentially longer-lasting, which should decrease the DRE. This issue does not appear to have greatly influenced the DRE onset results, since it would for example have added to an even greater difference between the slow and fast ramped onsets. However, there was no real difference between the DRE in these conditions.

Overall, the results presented here are thus in line with the previous experiments and the postulated double-inhibition model. Methodological constraints do limit the interpretation for reasons provided above, so further testing of the notion that robust stimulus offsets indeed provide an inhibitory boost which can overpower a salient but constant stream of sensory input would be of interest. We thus performed even more rigorous tests of the model in the next and final experiment. 



\section{Characterizing the disrupted rivalry}

\section{effect (III): "true" disrupted rivalry}

Related publication(s):

De Graaf, TA, van Ee, R*, Goebel, R, Sack, AT (in preparation). 'True disrupted rivalry': lasting visual suppression by interocular stimulus offset.

* Raymond van Ee

1) Department of Physics of Man, Helmholtz Institute, Utrecht University, Utrecht 3584 $\mathrm{CH}$, The Netherlands

2) Department of Brain, Body, Behaviour, Philips Research Laboratories, Eindhoven 5656 AE, The Netherlands

3) Department of Experimental Psychology, Leuven University, Leuven B-3000, Leuven, Belgium 


\begin{abstract}
In a binocular rivalry paradigm, the DRE phenomenon previously involved an adaptation phase of one stimulus in one eye, followed by flash suppression of a competing stimulus in the other eye, and quick removal of this flashed (then dominant) stimulus. The effect constituted in a lasting suppression (up to several seconds) of the only remaining, salient stimulus. On the basis of previous experiments we suggested that the abrupt stimulus offset itself inhibits the competing eye channel demonstrating that visual change in an eye channel can trump salient yet constant sensory inputs in the other eye channel. But this 'doubleinhibition' interpretation predicts that just an offset of a rivaling stimulus should suffice to suppress the remaining percept. To test this hypothesis, we implemented a 'true' disrupted rivalry paradigm, in which each trial began with standard binocular rivalry but one of the competing stimuli was removed after a variable delay. Based on continuous subjective reports of ongoing perception, trials were sorted post-hoc based on whether the removed stimulus had been dominant or suppressed. In $\sim 37 \%$ of trials there was no DRE at all. But when DRE was present, it was as strong as in previous experiments. Interestingly, not only was DRE present equally often when the removed stimulus had been dominant or suppressed, but its duration was actually longer when the suppressed stimulus had been removed. This is in line with our previous interpretation scheme of 'double-inhibition', but suggests that the contribution of stimulus offset is even larger than expected.
\end{abstract}




\section{Introduction}

When one eye is presented with an image, but the other eye is shown a competing image in the corresponding retinotopic location, conscious perception will switch between the two. This situation of binocular rivalry has become a popular research paradigm (Blake, 2005; Blake \& Logothetis, 2002). Under the umbrella of binocular rivalry phenomena fall also continuous flash suppression (Tsuchiya \& Koch, 2005; Tsuchiya, et al., 2006), flash suppression (Wolfe, 1984), flash facilitation (Brascamp, et al., 2007), possibly generalized flash suppression (Wilke, et al., 2003), and now perhaps an effect we have referred to as the disrupted rivalry effect (DRE). In DRE, one eye adapts to a stimulus, until the other eye is presented with a fresh competing stimulus. The second image immediately gains dominance (= flash suppression, see Wolfe, 1984), until perception again switches (van Ee, 2011). However, we found that quick removal (after hundred of milliseconds) of the flashed stimulus does not revert conscious percept back to the remaining percept, although salient and now without real competition. Surprisingly, the salient stimulus can remain suppressed for up to several seconds in DRE.

One interpretation of this phenomenon involves a simplified model of mutual inhibition, in which each stimulus (eye of origin and/or representation) inhibits the other. When a stimulus becomes dominant, it overpowers the competing stimulus. Yet, during this suppression period it can recover, while the dominant stimulus fatigues. Thus, the scales tip and the process reverses. Flash suppression in this scenario may provides a strong boost of inhibition of the already fatigued previously dominant stimulus, ensuring stable dominance of the flashed stimulus (although the mechanisms underlying flash suppression are not necessarily identical to those involved in binocular rivalry: (Tsuchiya, et al., 2006)). DRE, then, may add to this a second boost of inhibition by the stimulus offset, which quickly follows the onset. This doubleinhibition account makes several predictions. In the previous chapter we confirmed that gradual, versus abrupt, stimulus onsets/offsets decreased the DRE duration. We also, however, considered that DRE may not be fully subserved by rivalry between eyechannels alone.

In the current study, we tested one further prediction of our double-inhibition interpretation. The scenario suggests that stimulus offset, by itself, should also result in DRE, using the stimuli and setup we implemented. Moreover, the model makes the rather strong and counterintuitive prediction that DRE could be as long-lasting or actually longer-lasting when suppressed stimuli are removed in a situation of binocular rivalry, as when dominant stimuli are removed. After all, when the removed stimulus was suppressed that eye-channel will already have been recovering and therefore in a stronger position if it should become dominant. The stimulus offset provides the visual change that favors the thus-far suppressed eye-channel. This seems counterintuitive, since the brain has not even selected the stimulus for perception when it is removed: the easiest thing would be to just continue the ongoing percept that, after all, remains in view.

We therefore adapted the paradigm to a 'true disrupted rivalry' paradigm. On every trial, subjects were immediately presented with two competing stimuli in one 
visual field location. Then, after a variable delay, one of the two stimuli was removed. As subjects continuously recorded their perception (of 'star', 'triangle', or no percept), we could post-hoc determine whether on given trials the currently dominant or currently suppressed stimulus was removed. We analyzed the presence and duration of DRE on dominant and suppressed trials, and analyzed the effect of duration of dominance (or suppression) of a stimulus up to the point of removal on the duration of DRE.

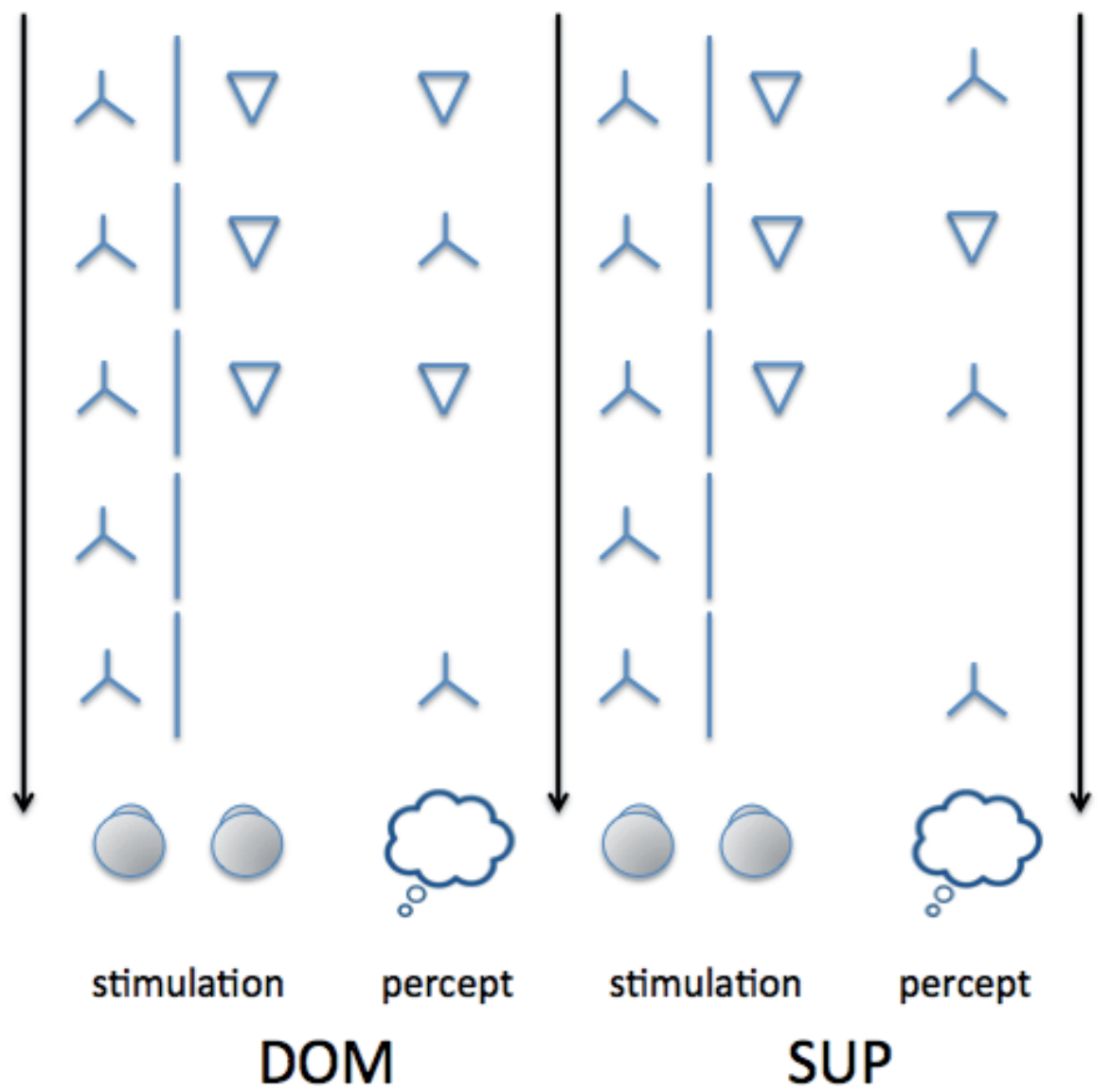

Figure 1: Main conditions and corresponding percepts

Shown are the stimulation protocol and corresponding percepts in the two main conditions: DOM (dominant: the stimulus element that was pseudo-randomly removed was dominant at the time of removal) and SUP (suppressed: the stimulus element that was removed was suppressed (i.e. not perceived) at the time of removal). In terms of stimulation parameters, there were no relevant main conditions: on any given trial both stimuli would be presented, participants continuously reported which was perceived. If neither was perceived, they would release both response keys. By these subjective reports, we could post-hoc sort the trials into DOM and SUP. The duration of rivalry until removal of one stimulus was varied between 4000-9000 milliseconds, to increase the chances of obtaining an even division of trials between both conditions. Indeed, there were approximately an equal number of trials in DOM and SUP. 


\section{Materials and Methods}

\section{Participants}

Ten participants volunteered for this experiment. Eight were naive to the goals and content of the study, nine were untrained in binocular rivalry paradigms and maintaining fixation. All had normal or corrected-to-normal vision and provided written informed consent. The study was approved by the local ethics committee.

\section{Stimuli, Tasks, and Design}

Stimuli consisted of 'blank', 'star' or 'triangle' images, presented separately to the left and right eye. All these contained a central fixation dot and a peripheral frame to aid in fixation and binocular fusion. Stimuli were identical to chapters I.1 (experiments 2-4) and I.3, as was the experimental setup.

The task for subjects was to continually track and record their conscious percepts. They used a standard computer mouse, pressing and holding the left mouse button with right index finger for as long as they perceived triangle elements and pressing and holding the right mouse button with right middle finger for as long as they perceived star elements. By default and explicit instruction, if no star or triangle were perceived, this required the release of both mouse buttons. This allowed us to post-hoc sort trials (using custom scripts in Matlab software) into two conditions. Dominant (DOM) trials were trials in which, on the basis of subjective reports up to the point of stimulus removal, the removed stimulus was at the time perceived. Suppressed (SUP) trials were trials in which the removed stimulus was at the time suppressed by the competing stimulus. See Figure 1 for a schematic visualisation of trials, stimulation and corresponding percepts.

Trials in which the post-hoc sorting process was not unequivocal (e.g. participants had pressed both mouse buttons simultaneously for a prolonged period, indicated switched perception in the $300 \mathrm{~ms}$ after stimulus removal [i.e. taking into account response time this means perceptual switches occurred around stimulus removal], or otherwise did not follow instructions properly on a trial) were removed prior to analysis. Each trial began with simultaneous presentation of both competing stimuli, star/triangle stimuli randomly assigned to right/left eye but balanced over the experiment. After a delay of either 4000, 5000, 6000, 7000, 8000, 9000 milliseconds, star or triangle stimulus would (randomly, but balanced) be removed (i.e. replaced with blank image). The trial would then continue for 4500 milliseconds, which means that the maximum effect duration measured by our design was under $4500 \mathrm{~ms}$. A total number of 48 trials per participants were measured per run. Four runs separated by breaks (lights on, freedom to move, self-paced) were measured per participant in one session. As the conditions of DOM and SUP were determined post-hoc their respective numbers of trials will be reported in Results. 


\section{Analyses}

No outliers were removed since the maximum effect duration was ceilinged to $4500 \mathrm{~ms}$ by design and it was an open question whether or not any effects would occur and under which conditions. However, one participant was removed prior to analysis because he/she consistently failed to properly release one of the two mouse buttons indicating the two possible percepts, making it impossible on a great number of trials to determine which images were perceived at which time. All other participants followed this instruction properly and could be included.

Repeated-measures ANOVAs (RM-ANOVAs; Huynh-Feldt corrected) evaluated the proportions of trials with DRE effects for DOM and SUP conditions. On trials with DRE effects, RM-ANOVAs compared the time from stimulus element removal that participants indicated a lack of any percept (Percept End: P-E) and the time that they indicated seeing again the only remaining stimulus element (Percept Return: P-R). P-E was taken as a form of baseline to compare the P-R (essential DRE) response times to. With P-E/P-R and DOM/SUP, this constituted a 2x2 RM-ANOVA. Any follow-up tests as reported in Results.

We aimed to evaluate (over all trials over all participants) separately for DOM and SUP trials whether there was a relation between the percept duration before stimulus removal and duration of potential DRE. For this, we extracted the time of dominance or suppression up to the point of stimulus removal (Percept Before removal $=\mathrm{P}-\mathrm{B})$, and the P-R (=DRE). To correlate these values for individual trials over all subjects we first standardized these values based on subject- and condition-specific means and standard deviations. We then used Pearson's correlation (2-tailed test for significance).

\section{Results}

Post-hoc sorting of trials into dominant (DOM) or suppressed (SUP) conditions on the basis of their reported percept at the moment of pseudorandomized stimulus element removal resulted in an equal number of trials in both conditions. On average, 82.3 trials were measured per participant in DOM, and 81.6 in SUP. There was no significant difference $(\mathrm{P}=0.874)$. The first question was how many of these trials also led to a DRE. In contrast to our previous disrupted rivalry experiments, there could now really be no DRE effect at all, if participants just held the same button or immediately switched to the alternative button (depending on whether dominant or suppressed stimulus was removed). Figure 2 shows for both DOM and SUP conditions the proportions of trials averaged over participants in which a DRE was reported. Indeed, participants reported seeing neither stimulus element in quite a few trials, $65.3 \%$ of trials for DOM and $60.5 \%$ of trials for SUP. Over participants, this was not significantly different $(\mathrm{P}=0.305)$.

We proceeded to evaluate the durations of DRE in the trials in which there was a reported disappearance of the only remaining stimulus element. Figure 3 shows these durations $(\mathrm{P}-\mathrm{R}$ : percept return $=$ time since stimulus element removal that the remaining percept becomes again visible), and provides as a form of baseline the P-E (percept-end 
$=$ time since stimulus element removal before absence of conscious perception is reported). Since P-E is much less predictable (due to the large variation in timing since trial onset that stimulus removal occurred) than P-R, it is not a very reliable indication of 'general' response time. But it does constitute a useful individual baseline to exclude overly long reaction times as an explanation op DRE in this experiment, and to form a backdrop to compare the durations of DRE to.

\section{Proportion of trials with DRE}

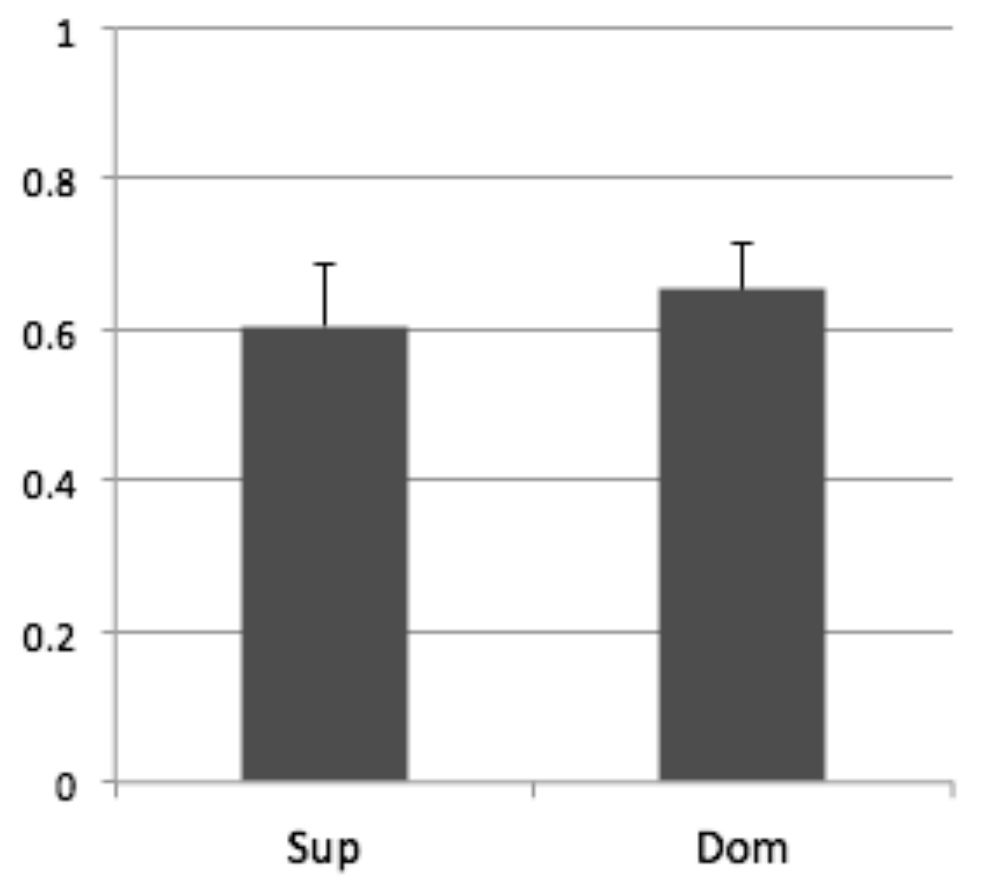

Figure 2: proportion of trials with DRE.

Shown separately for SUP (removed stimulus was suppressed) and DOM (removed stimulus was dominant) are the proportions of trials with disrupted rivalry effects (reported disappearance of stimuli). The proportion of trials appears slightly higher for DOM, but the difference was not significant. Within-subject error bars (Cousineau, 2005).

A RM-ANOVA with factors DRE (P-E/P-R) and Perc (DOM/SUP) showed a main effect of DRE $(\mathrm{F}=107.6 ; \mathrm{P}<0.001)$, demonstrating that even without the adaptation phase (i.e. in a case of 'true' disrupted rivalry) the DRE can occur. Interestingly, there was also a main effect of Perc $(\mathrm{F}=14.2 ; \mathrm{P}<0.01)$, showing that it does make a difference whether the removed stimulus was at the time perceived or not. A priori, it should not make a difference for the response time baseline P-E, so this effect would likely be 


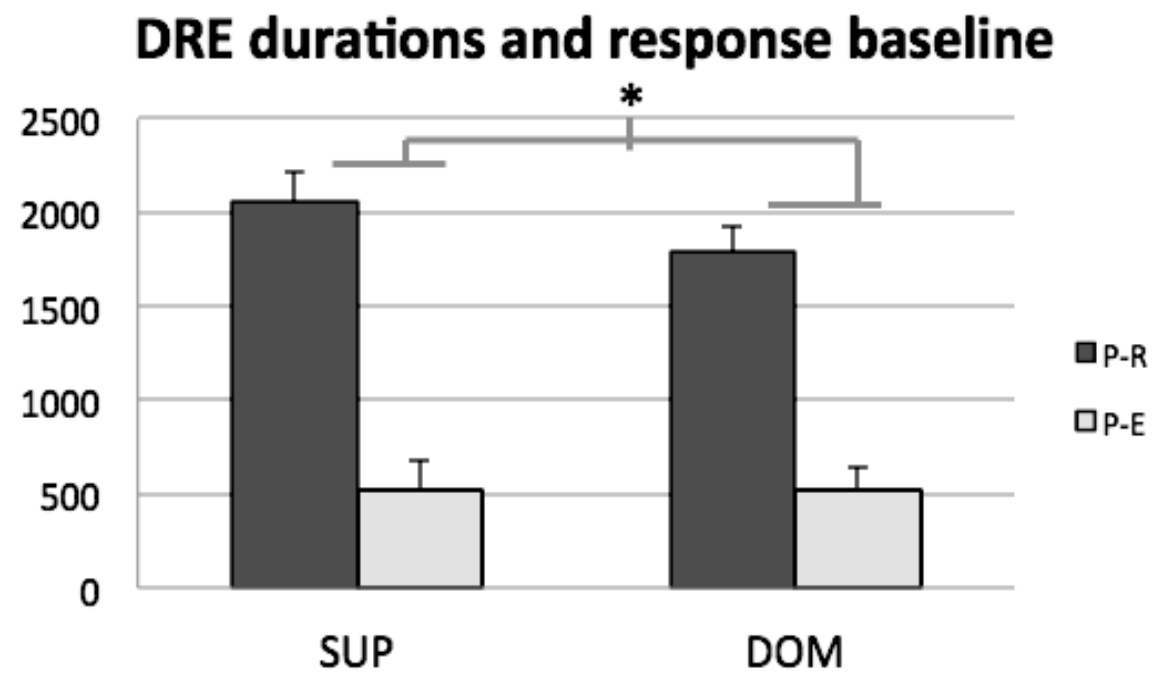

Figure 3: DRE durations and response time baseline

Shown separately for SUP (removed stimulus element was suppressed) and DOM (removed stimulus element was dominant) are the time from stimulus element removal to the subjective report of perception of the remaining stimulus element, in dark grey (P-R: percept-return). For comparison, P-E (response time to the removal of the stimulus element) is shown in light grey, as a baseline for reaction times in the subjective report setting. As a direct measure of $\mathrm{RT}$ in the P-R condition it is not wholly adequate (it is too stringent, see main text), but it does provide an individual baseline to relate the DRE durations to. Interestingly, the DRE duration seems to be longer in the SUP condition, and as indicated by the asterisk, there is in fact a significant interaction between DOM/SUP and P-R, P-E. Within-subject error bars (Cousineau, 2005)

attributable to P-R only. Indeed, there was a significant interaction between DRE x Perc $(\mathrm{F}=14.8 ; \mathrm{P}<0.01)$. Follow-up tests (2-tailed, bonferroni-corrected) showed that there was a significant difference between DOM and SUP for the DRE (P-R) condition $(\mathrm{t}(8)=3.9 ; \mathrm{P}<0.01)$ but not for $\mathrm{P}-\mathrm{E}(\mathrm{P}=0.749)$. As outlined in the Introduction, although counterintuitive, we could thus confirm that the effect of DRE is even longer-lasting when suppressed visual stimuli are removed as compared to removal of currently dominant stimuli.

The double-inhibition model also predicts that the duration of DRE should depend on the time that the dominant or suppressed stimulus had been dominant or suppressed at the moment of stimulus removal. Specifically, if the removed stimulus had been dominant at the time, the longer it had been dominant, the shorter the DRE should last. We should thus expect a negative correlation. In contrast, if the removed stimulus had been suppressed at the time, the longer it had already been suppressed, the longer the DRE should last. Here we should expect a positive correlation. Post-hoc, we could extract the time that SUP or DOM removed stimuli had been suppressed or dominant respectively (Percept Before removal: P-B), standardize these values (see Methods) and correlate these values with the standardized durations of DRE over all trials of all participants. As hypothesized, there was a significant negative correlation 
$(\mathrm{r}=-0.204 ; \mathrm{P}<0.001)$ between $\mathrm{P}-\mathrm{B}$ and $\mathrm{P}-\mathrm{R}$ (i.e. DRE) when the removed stimulus had been dominant (DOM). In contrast, and also in accordance with the double-inhibition model, there was a significant positive correlation $(\mathrm{r}=0.151 ; \mathrm{P}=0.001)$ between $\mathrm{P}-\mathrm{B}$ and P-R (i.e. DRE) when the removed stimulus had been suppressed (SUP).

\section{Discussion}

In previous experiments we established the disrupted rivalry effect (DRE) and constrained its underlying mechanisms. This led us to propose a double-inhibition account in a simple mutual-inhibition between eye-channels model. Yet, the previous chapter showed that it seems primarily the stimulus offset that affects the duration of DRE, as gradual offsets (as compared to abrupt offsets) led to the strongest decrease in DRE. So, rather than focusing on our paradigm as a 'double'-inhibition, perhaps the most striking feature of the implementation is the 'offset-inhibition'.

This interpretation led to the hypothesis that 'true disrupted rivalry', in which standard binocular rivalry would be disrupted by the removal of the dominant stimulus, should also lead to the DRE. In fact, counterintuitively this account predicted that removal of even the suppressed stimulus should also lead to DRE. This seems somewhat unlikely, since that would amount to the scenario that subjects are seeing one stimulus and not the other - yet when the other, unseen stimulus is actually removed, the currently dominant stimulus would suddenly become invisible. This is, however, exactly what we observed here. Moreover, our previous interpretation would even predict that DRE might be stronger when the removed stimulus was suppressed, as opposed to currently dominant, since suppression at the onset of the DRE phase essentially amounts to a rivalry time of 0 (rivalry time was in previous experiments shown to negatively relate to DRE duration). By the same logic, if dominant stimuli are removed, the longer the dominance duration up to the point of removal, the shorter the effect should be (i.e. essentially this amounts to a longer rivalry time). Yet, for suppressed stimuli, the longer the suppression duration up to the moment of removal, the longer the effect should last. After all, if the relative strength of the currently dominant stimulus has already been reduced by a prolonged dominance period, the offset-induced inhibitory boost will bring it to an even lower level.

All these predictions were confirmed by our data. True disrupted rivalry, without an adaptation and flash suppression phase, did still result in DRE. This was the case for dominant and suppressed removals equally often, and DRE was stronger for suppressed removals. Lastly, for dominant trials there was a negative correlation between the time removed stimuli had been dominant and DRE, and for suppressed trials there was a positive correlation between the time removed stimuli had been suppressed and DRE.

There are some additional interesting aspects of this dataset worth mentioning. One, in contrast to our experiences with the 'standard' disrupted rivalry paradigm involving adaption phase and flash suppression (although that design could not exclude trials without effects, informal observations suggested that the effect was always present), there was not always a DRE in the current 'true disrupted rivalry' paradigm, although more often than not. On trials were there was no DRE, participants just 
continued to see the dominant stimulus or immediately reverted to seeing the remaining previously suppressed stimulus. Also informally, we observed that occasionally a trial just ended as if nothing had happened. But when there was a DRE, it was strong. In fact, it was as strong in this experiment, with only the offset-phase of our 'doubleinhibition' account (i.e. now a 'single-inhibition' scenario), as it was in previous experiments. Thus, IF the brain is fooled into suppressing the eye-channel that is presented with meaningful and salient information, it is fooled for up to several seconds.

Since a potentially inhibiting stimulus onset was lacking in the current paradigm, it seems that the stimulus offset may be the defining factor in DRE duration. This is in concert with the finding in previous chapters that a slow gradual stimulus offset (as opposed to onset) in the DR paradigm leads to the strongest reduction in DRE. Yet, there were fewer trials in which DRE occurred at all in the current study, which may suggest that the inhibition from flashed stimulus onset does help to put the brain into a state in which one eye channel, or the peripheral percept, is suppressed. The duration of this suppression may then in large part be determined by the rate of stimulus offset (i.e. visual change). Although note that the current experiment also shows that the flashed stimulus onset is not necessarily required to achieve DRE.

A full explanation of the collective set of results might involve an interaction between various mechanisms underlying stimulus suppression, such as fading (Kanai \& Kamitani, 2003; May, et al., 2003; Troxler, 1804), generalized flash suppression (Wilke, et al., 2003) or filling-in (Pessoa \& de Weerd, 2003). In the General Discussion we discuss the link to potentially related mechanisms in more detail, but in the current experiment specifically it is of note that DRE was either present or not present, but when present it was in full force. From the double-inhibition account only we should expect that having here only 'single-inhibition', the average duration of DRE should be somewhere between 0 and the effect size in previous experiments. It thus seems wholly possible that the double-inhibition (i.e. rivalry) account of DRE tells only part of the story. It could help put the brain in a from of local attractor state where one eye-channel is suppressed, after which the stimulus continues to remain 'invisible' due to contributions of fading, filling-in, and potentially additional factors. These are the subject of future investigations. 


\section{General discussion: the disrupted rivalry effect}

There are a few general remarks we should make about the disrupted rivalry effect (DRE) at this point. Primarily, we briefly discuss how the effect may relate to previous work, and secondarily, we consider the effect duration.

\section{DRE and previous work}

In the general discussion of chapter I.1, we outlined some of the proposed neural dynamics underlying DRE. In following chapters we aimed to test these propositions and build on the effect and the model. Here, we note that DRE may relate to several previous observations. One component of DRE that seems of interest is the proposal that it is an eye-channel that is suppressed (at one or more cortical locations along monocular pathways; (Blake, 1989; Blake \& Logothetis, 2002; Freeman, et al., 2005). It has been noted before that the scope of visual suppression in a state of binocular rivalry is rather broad. Although quick alternation of which of two stimuli is presented to which eye can result in 'normal' bistable perception characteristics (suggesting that rivalry can take place on the level of stimulus representation rather than eye: (Logothetis, et al., 1996), simply switching the stimuli once between both eyes can lead to an immediate switch in percept (Blake, Westendorf, \& Overton, 1980). The latter finding is an example of eye-suppression, in which the 'contents' of suppression are irrelevant; it is the eye-channel that continues to be suppressed (or dominant). The surprisingly long suppression of an eye channel in DRE, even without 'true' interocular competition, may relate in this respect to work by Blake et al. (1990). These authors employed standard binocular rivalry, but then forced a stimulus to regain dominance immediately after it was suppressed. Results showed that while the thus regained dominance periods were unusually short, the following suppression periods were longer than usual, supporting proposed contributions of local adaptions/fatigue to suppression period durations.

Although thus related to binocular rivalry, DRE may also relate to two previous effects that demonstrate disappearance of visual targets without interocular conflict. First, it has been shown that visual transients can induce Troxler fading. Kanai \& Kamitani (2003) presented peripheral visual targets, and showed that 1) a flashed image surrounding the target, 2) an apparent motion setting, 3) a brief 'blink' of the target itself, could all induce time-locked perceptual fading. Although in chapter I.2 we compared the frequency and duration of 'true fading', the fact that such visual transients could induce a fading spell reinforces our interpretation that visual change is a dominant force in the selection of visual inputs for conscious vision. In line with this, Kanai et al. (2005) demonstrated that visual transients could also induce perceptual alternations in several bistable paradigms, including binocular rivalry.

A series of experiments by Wilke et al. (2003) established a different paradigm to abolish a salient visual stimulus from visual consciousness. They developed 'generalized flash suppression' (GFS). A peripheral target was presented to one eye (and a 'blank' to the other eye) for a duration of minimally a few hundred milliseconds. 
As opposed to conventional flash suppression, an array of stationary or moving dots (or several additional visual manipulations in following experiments) were presented around the visual target, to the same eye, to the opposite eye, or to both eyes. Particularly when these surrounding visual transients were presented to the opposite eye (or both eyes), the visual target would often disappear from conscious vision for several seconds (analogous to DRE). Since GFS also involved visual targets presented to one eye without 'true' interocular competition, the mechanisms underlying this effect may relate to DRE.

The fundamental difference between DRE and these potentially related observations is that DRE involves the instigation of visual suppression of a very salient target (in contrast to the time-locked fading stimulus of Kanai \& Kamitani, 2003) by the offset of an interocularly competing stimulus. Thus, in DRE, the full period of visual suppression involves a situation of 1) no visual stimulation, 2) no interocular competition, 3) instigation of disappearance by stimulus offset rather than onset. Nevertheless, as it has been suggested that visual suppression in bistable perception and fading (and soforth) may share to some extent underlying neural dynamics (Y. S. Bonneh, et al., 2001; Carter \& Pettigrew, 2003; Kanai, et al., 2005), further work aiming to distill these contributions would be valuable.

\section{The potential suppression duration}

In the majority of the experiments reported here, we probably underestimated the potential effect duration. We used primarily untrained participants, who were unfamiliar with binocular rivalry, untrained in fixation, and unaware of the intents and purposes of the experiment. While we emphasized the importance of properly maintaining fixation, without any real motivation other than fulfillment of course credit or monetary coupons - neither of which were in any way tied to performance - it seems highly plausible that saccades did contaminate a great number of trials. This immediately ended the effect, in line with established findings that abruptly changing the contrast of (Wilson, et al., 2001) or moving (Walker \& Powell, 1979) suppressed visual stimuli immediately breaks suppression. Some experimental sessions were quite long, and none were particularly challenging or otherwise interesting to participants, so the task would be fatiguing. One solution for future studies would be to links rewards to the successful maintenance of fixation, but this would require online eye-tracking in the stereoscope setup, which at this point was not feasible in our lab. Other solutions to maximize sensitivity of the design would be to train participants in our setup first, or recruit trained fixators, and implement shorter experimental sessions.

Another factor limiting the effect duration, possibly interacting with fatigue and motivation as well, may have been the use of the single stimulus element. For establishing the effect, this seemed to be the clearest and most controlled stimulus. However, in pilot measurements using whole-field arrays of stimuli, we noticed effect durations of around 4-5 seconds rather than 1.5 - 2 seconds. The single stimulus element of course draws all the attentional resources. It makes it also very hard to continue fixating, as it is not so much 'pure black' that is perceived during the effect. While difficult to explain, for the observer it seems apparent that 'something is there'. 
This visual impression that something is in one's periphery that one cannot see creates a very strong urge to make a saccade to the area. The experimental session was thus a continuous battle to inhibit this urge. One possible solution for this in future studies may be the use of two stimulus elements, on opposite sites of the fixation point. This would require divided attention to two opposite locations, hopefully diminishing the urge to saccade to either one in particular.

One shortcoming of the series of experiments presented here is the consistent use of one stimulus. We used triangles and stars throughout the experiments, to allow continued comparison and integration of results. Our stimuli were 'thin and long', consisting in extended high-contrast boundaries to the black background and only very limited surface. It would be of interest to compare the frequency and extent of DRE for stimuli with larger surfaces and more complex stimuli. 


\section{Part 1 References}

Alais, D., Cass, J., O'Shea, R. P., \& Blake, R. (2010). Visual sensitivity underlying changes in visual consciousness. Curr Biol, 20(15), 1362-1367.

Bartels, A., Vazquez, Y., Schindler, A., \& Logothetis, N. K. (2011). Rivalry between afterimages and real images: the influence of the percept and the eye. J Vis, 11(9).

Blake, R. (1989). A neural theory of binocular rivalry. Psychol Rev, 96(1), 145-167.

Blake, R. (2005). Landmarks in the History of Binocular Rivalry. In D. Alais \& R. Blake (Eds.), Binocular Rivalry (pp. 1-28). Cambridge, MA: A Bradford Book, The MIT Press.

Blake, R., \& Logothetis, N. K. (2002). Visual competition. Nat Rev Neurosci, 3(1), 1321.

Blake, R., Westendorf, D., \& Fox, R. (1990). Temporal perturbations of binocular rivalry. Percept Psychophys, 48(6), 593-602.

Blake, R., Westendorf, D. H., \& Overton, R. (1980). What is suppressed during binocular rivalry? Perception, 9(2), 223-231.

Bonneh, Y., Sagi, D., \& Karni, A. (2001). A transition between eye and object rivalry determined by stimulus coherence. Vision Res, 41(8), 981-989.

Bonneh, Y. S., Cooperman, A., \& Sagi, D. (2001). Motion-induced blindness in normal observers. Nature, 411(6839), 798-801.

Brascamp, J. W., \& Blake, R. (2012). Inattention Abolishes Binocular Rivalry: Perceptual Evidence. Psychol Sci.

Brascamp, J. W., Knapen, T. H., Kanai, R., van Ee, R., \& van den Berg, A. V. (2007). Flash suppression and flash facilitation in binocular rivalry. J Vis, 7(12), 12 11-12.

Carter, O. L., \& Pettigrew, J. D. (2003). A common oscillator for perceptual rivalries? Perception, 32(3), 295-305.

Chen, X., \& He, S. (2003). Temporal characteristics of binocular rivalry: visual field asymmetries. Vision Res, 43(21), 2207-2212.

Cousineau, D. (2005) Confidence intervals in within-subject designs: A simpler solution to Loftus and Masson's method, Tutorials in Quantitative Methods for Psychology, 1, 75-78

Freeman, A. W., Nguyen, V. A., \& Alais, D. (2005). The Nature and Depth of Binocular Rivalry Suppression. In D. Alais \& R. Blake (Eds.), Binocular Rivalry (pp. 47-62). Cambridge, MA: A Bradford Book, The MIT Press.

Gilroy, L. A., \& Blake, R. (2005). The interaction between binocular rivalry and negative afterimages. Curr Biol, 15(19), 1740-1744.

Kanai, R., \& Kamitani, Y. (2003). Time-locked perceptual fading induced by visual transients. J Cogn Neurosci, 15(5), 664-672.

Kanai, R., Moradi, F., Shimojo, S., \& Verstraten, F. A. (2005). Perceptual alternation induced by visual transients. Perception, 34(7), 803-822.

Kang, M. S., \& Blake, R. (2010). What causes alternations in dominance during binocular rivalry? Atten Percept Psychophys, 72(1), 179-186.

Lehky, S. R. (1988). An astable multivibrator model of binocular rivalry. Perception, $17(2), 215-228$. 
Leopold, D. A., \& Logothetis, N. K. (1996). Activity changes in early visual cortex reflect monkeys' percepts during binocular rivalry. Nature, 379(6565), 549-553.

Levelt, W. (1965). On Binocular Rivalry. Institute for Perception RVOTNO, Soesterberg, Netherlands.

Logothetis, N. K., Leopold, D. A., \& Sheinberg, D. L. (1996). What is rivalling during binocular rivalry? Nature, 380(6575), 621-624.

Macknik, S. L., \& Livingstone, M. S. (1998). Neuronal correlates of visibility and invisibility in the primate visual system. Nat Neurosci, 1(2), 144-149.

Macknik, S. L., Martinez-Conde, S., \& Haglund, M. M. (2000). The role of spatiotemporal edges in visibility and visual masking. Proc Natl Acad Sci USA, 97(13), 7556-7560.

May, J. G., Tsiappoutas, K. M., \& Flanagan, M. B. (2003). Disappearance elicited by contrast decrements. Percept Psychophys, 65(5), 763-769.

Mueller, T. J., \& Blake, R. (1989). A fresh look at the temporal dynamics of binocular rivalry. Biol Cybern, 61(3), 223-232.

Noest, A. J., van Ee, R., Nijs, M. M., \& van Wezel, R. J. (2007). Percept-choice sequences driven by interrupted ambiguous stimuli: a low-level neural model. $J$ Vis, 7(8), 10.

Pessoa, L., \& de Weerd, P. (Eds.). (2003). Filling-In: From Perceptual Completion to Cortical Reorganization. USA: Oxford University Press.

Ramachandran, V. S., \& Gregory, R. L. (1991). Perceptual filling in of artificially induced scotomas in human vision. Nature, 350(6320), 699-702.

Roland, P. E. (2010). Six principles of visual cortical dynamics. Front Syst Neurosci, 4, 28.

Simons, D., Lleras, A., Martinez-Conde, S., Slichter, D., Caddigan, E., \& Nevarez, G. (2006). Induced visual fading of complex images. $J$ Vis, 6(10), 1093-1101.

Sobel, K. V., \& Blake, R. (2002). How context influences predominance during binocular rivalry. Perception, 31(7), 813-824.

Tong, F., Meng, M., \& Blake, R. (2006). Neural bases of binocular rivalry. Trends Cogn Sci, 10(11), 502-511.

Troxler, D. (1804). In K. Himly \& J. A. Schmidt (Eds.), Ophthalmologische Bibliothek (Vol. 2, pp. 1-119). Jena: Fromann.

Tsuchiya, N., \& Koch, C. (2005). Continuous flash suppression reduces negative afterimages. Nat Neurosci, 8(8), 1096-1101.

Tsuchiya, N., Koch, C., Gilroy, L. A., \& Blake, R. (2006). Depth of interocular suppression associated with continuous flash suppression, flash suppression, and binocular rivalry. $J$ Vis, $6(10), 1068-1078$.

van Ee, R. (2011). Percept-switch nucleation in binocular rivalry reveals local adaptation characteristics of early visual processing. $J$ Vis, 11(2).

Walker, P., \& Powell, D. J. (1979). The sensitivity of binocular rivalry to changes in the nondominant stimulus. Vision Res, 19(3), 247-249.

Whittle, P., Bloor, D. C., \& Pocock, S. (1968). Some experiments on figural effects. Perception and Psychophysics, 4, 183-188.

Wilke, M., Logothetis, N. K., \& Leopold, D. A. (2003). Generalized flash suppression of salient visual targets. Neuron, 39(6), 1043-1052. 
Brain in Sight

Wilson, H. R., Blake, R., \& Lee, S. H. (2001). Dynamics of travelling waves in visual perception. Nature, 412(6850), 907-910.

Wolfe, J. M. (1984). Reversing ocular dominance and suppression in a single flash. Vision Res, 24(5), 471-478. 
Part II

Low-level Aspects of Vision:

TMS Masking 

II.1

\title{
A Chronometric Exploration of High-
}

resolution 'Sensitive TMS Masking'

\author{
Effects on Subjective and Objective
}

Measures of Vision

Related publication(s):

De Graaf, TA, Herring, J, Sack, AT (2011). A chronometric exploration of highresolution 'sensitive TMS masking' effects on subjective and objective measures of vision. Experimental Brain Research. 209:19-27 


\begin{abstract}
Transcranial magnetic stimulation (TMS) can induce masking by interfering with ongoing neural activity in early visual cortex. Previous work has explored the chronometry of occipital involvement in vision by using single pulses of TMS with high temporal resolution. However, conventionally TMS intensities have been high and the only measure used to evaluate masking was objective in nature. Recent studies have begun to incorporate subjective measures of vision, alongside objective ones. The current study goes beyond previous work in two regards. First, we explored both objective vision (an orientation discrimination task) and subjective vision (a stimulus visibility rating on a four-point scale), across a wide range of time windows with high temporal resolution. Second, we used a very sensitive TMS-masking paradigm: stimulation was at relatively low TMS intensities, with a figure- 8 coil, and the small stimulus was difficult to discriminate already at baseline level. We hypothesized that this should increase the effective temporal resolution of our paradigm. Perhaps for this reason, we are able to report a rather interesting masking curve. Within the classical-masking time window, previously reported to encompass broad SOAs anywhere between 60 and $120 \mathrm{~ms}$, we report not one, but at least two dips in objective performance, with no masking in-between. The subjective measure of vision did not mirror this pattern. These preliminary data from our exploratory design suggest that, with sensitive TMS masking, we might be able to reveal visual processes in early visual cortex previously unreported.
\end{abstract}




\section{Introduction}

Studies into visual awareness often adopt masking paradigms. In such paradigms, a visual target stimulus is presented briefly, and a secondary visual stimulus (mask) is presented either before or after the target stimulus. By using different stimulus onset asynchronies (SOAs) between target and mask, the level of processing of the visual target can be modulated (Breitmeyer and Ogmen 2006). This indicates that there is a temporal organization in visual cortex that underlies the establishment of conscious vision. At certain points in time, this organization should be left unperturbed, or vision will be abolished.

To target the organization in early visual regions specifically, a brain interference method can be used. Transcranial magnetic stimulation (TMS) is ideal in this regard, because it affords a high temporal resolution in brain interference. Using TMS at different SOAs from a visual target stimulus has yielded different time windows at which the organization of stimulated early visual cortex should be left unperturbed (Kammer 2007a). Classically, a time window around 100ms from visual target stimulus onset was revealed (Amassian et al. 1989). But in addition, earlier time windows have been reported (Corthout et al. 1999; Paulus et al. 1999; Laycock et al. 2007) and TMS effects in later time windows as well (Heinen et al. 2005; Camprodon et al. 2010). When using longer-lasting motion stimuli and relating TMS to stimulus offset, again two masking periods were identified rather than one (Silvanto et al. 2005; Koivisto et al. 2010).

Most TMS-masking studies at any rate identified the 'classical' masking effect around $100 \mathrm{~ms}$. However, the precise latency at which masking was strongest, or even present, can differ between studies (Kammer 2007a, b). For instance, masking effects have been reported at 60-75 ms (Romei et al. 2007), 80-100 ms (Amassian et al. 1989), and $120 \mathrm{~ms}$ (Kammer et al. 2003). Thus, since it seems impossible to pinpoint chronometrically when a TMS pulse has its effect, a window spanning perhaps 60-120 $\mathrm{ms}$ is regarded to house a TMS-masking effect. We will continue to refer to this as the 'classical- masking time window'.

TMS-masking studies conventionally evaluate vision by an objective measure. If the research interest concerns visual awareness, one might argue that a subjective measure is appropriate. After all, a core aspect of visual consciousness is the subjective and reportable nature of it (Boyer et al. 2005; Lau and Passingham 2006; Koivisto et al. 2010). Moreover, the combination of both objective and subjective measures of vision may yield very interesting insights. Boyer et al. (2005) asked their subjects on every trial to discriminate the orientation of a bar and to moreover subjectively report whether they consciously perceived it or not. When evaluating objective discrimination performance on trials that were reported not to be consciously perceived, performance was still far above-chance. This has been dubbed 'TMS-induced blindsight'. That particular study applied TMS pulses only in the classical masking time window around $100 \mathrm{~ms}$. This finding raises the question of what happens in other time windows when both subjective and objective measures of vision are implemented. Are dissociations time-specific? How does unimpaired objective performance square with previous reports of strong objective TMS masking at this late time window? More generally: 
does objective visual performance reflect the subjective experience of the visual stimuli? Could both measures be used alternately, or in unison, to investigate visual consciousness? There is to date no study that performed a rigorous high-resolution exploration of the chronometry of TMS masking of subjective and objective vision. This was one goal of the current work.

The second goal was to evaluate what happens to the TMS-induced masking curve, over time, if the TMS-masking paradigm is made to be as sensitive as possible. The purported advantage of single-pulse TMS is the high temporal resolution (Wassermann et al. 2008). However, even if the magnetic field change of a single TMS pulse lasts under a millisecond, it does not mean the neural effects of that pulse are equally brief. In fact, TMS-induced differential effects on neuron behavior can last up to hundreds of milliseconds (Moliadze et al. 2003, 2005). The stronger the TMS pulse is, the longer these neural effects can be expected to last. Traditional TMS-masking studies used circular coils (e.g., Amassian etal. 1989; Beckers and Homberg 1991), and indeed they are yet often used (Boyer et al. 2005). The spatial extent of disturbance may affect how long the neural effects last as well. Indeed, as pointed out, most TMSmasking studies reveal masking in broad time windows around $100 \mathrm{~ms}$, rather than a single peak in one time window (say between 60 and $120 \mathrm{~ms}$, see above). A figure- 8 coil can at least provide focal stimulation, addressing this spatial resolution issue. It seems plausible that within a time window of tens of milliseconds early visual cortex is differentially involved in visual processing over this time, rather than occupied in the same way uniformly (see e.g., Foxe and Simpson 2002). The question is whether TMS masking is sensitive enough to reveal putative multiple stages of such involvement. One last factor that may affect the masking curve is the stimulus itself. Larger and clearer stimuli may need higher TMS intensities to yield any masking effect, since the information that needs to be suppressed is more salient. Higher intensities in turn may again decrease temporal resolution. Thus, a more sensitive paradigm might require nonsalient stimuli.

Altogether, these considerations lead us to hypothesize that, to obtain the highest effective temporal resolution, one should (1) use difficult and small visual target stimuli, (2) a spatially precise TMS coil, to allow masking with (3) the lowest TMS intensities possible (that still yield masking effects), and (4) high temporal sampling rate (minimizing gaps between measured SOAs). In the current study, we implemented such a 'sensitive TMS paradigm' and explored its effects on both objective and subjective visual processing.

\section{Materials and Methods}

\section{Participants}

Fourteen participants (four men) volunteered for this study. None had a history of neuropsychiatric disorders, and all had normal or corrected-to-normal vision. The 
experiment was approved by the local medical-ethical committee, and written informed consent was obtained before participation. An independent medical supervisor screened all participants for TMS experimentation safety prior to measurements, and participants were compensated with gift certificates. Since localization of TMS target site involved elicitation of phosphenes, the four participants in whom phosphenes could not be elicited reliably or in the proper visual field location were not tested in the experiment, leaving a total of ten included participants.

A

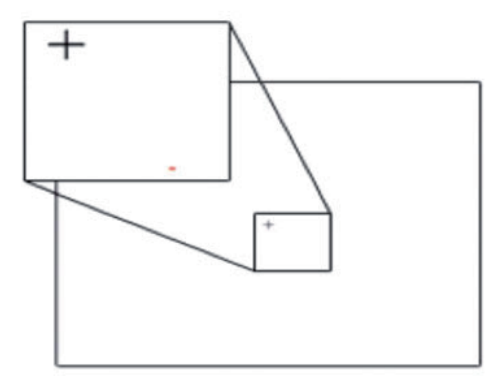

B

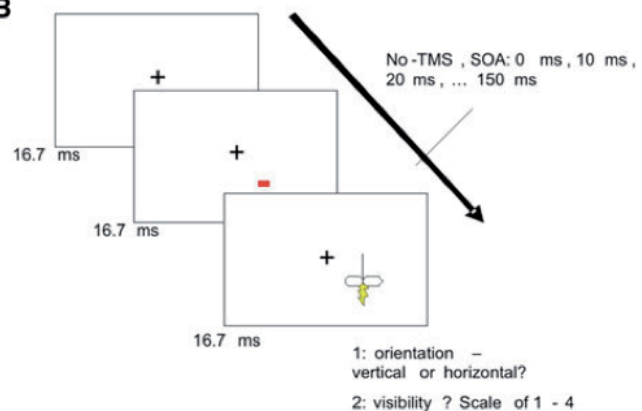

Figure 1. Stimulus and design.

A The stimulus was a horizontal or vertical bar. The stimulus was deliberately small and difficult to distinguish, in line with a 'sensitive TMS-masking paradigm' (see main text). B Stimuli were presented for $16.7 \mathrm{~ms}$ only, after which a TMS pulse at one SOA between 0 and $150 \mathrm{~ms}$ (except on no-TMS trials) was administered. Participants were asked to first make a forced-choice judgment about stimulus orientation. Second, they were asked to indicate on a scale of $1-4$ how clearly they perceived the orientation of the stimulus.

\section{Stimuli and tasks}

We used stimuli in the form of small rectangular bars $(\sim 0.025$ by $\sim 0.035$ visual degrees), that could be either horizontal or vertical in orientation, and either red or blue in color (for an example, see Fig. 1). Each stimulus was always presented for only 16.7 $\mathrm{ms}$, one frame on a monitor set to $60-\mathrm{Hz}$ refresh rate. Average luminance of the bars was $65 \mathrm{~cd} / \mathrm{m} 2$, of background $180 \mathrm{~cd} / \mathrm{m} 2$. The experiment involved a forced-choice discrimination task, and a subjective visibility rating task. In the objective task, subjects were required to indicate, using button presses, whether the orientation of the visual target stimulus was vertical (right index finger) or horizontal (right middle finger). These forced-choice discriminations were made first and constituted the objective measure of vision. After this judgment, participants were asked, in the same trial, to indicate subjectively the visibility of the stimulus. This was done using a 4-point scale, in which value ' 1 ' indicated "I didn't see the orientation at all"; ' 2 ' indicated "I don't think I saw the orientation"; ' 3 ' indicated "I think I did see the orientation"; " 4 ' indicated "I saw the orientation clearly". We indicated that these ratings were a subjective rating of how clearly the stimulus was perceived, not a confidence rating. 
Moreover, we emphasized that the descriptions were a guideline, but that consistency of the rating throughout the experiment was most important. Subjects were given ample time to practice with both discrimination tasks and subjective ratings.

Stimuli were presented on a standard TFT computer monitor (Samsung Syncmaster 931BF), using Presentation software (Neurobehavioral Systems, San Francisco, CA, USA). Viewing distance was $60 \mathrm{~cm}$. The location of the stimuli was 4 degrees visual angle to the lower left or right of fixation-depending on where participants perceived phosphenes more easily in the required eccentricity and polar angle (on the left side in three subjects). Subjects fixated throughout the experiment.

\section{Design and TMS parameters}

The experiment consisted of one session per subject. A total of 17 conditions (16 time windows + no-TMS) with 15 trials per time window resulted in 255 orientation task trials per session. Each trial took a minimum of 6 seconds, with subjects otherwise self-pacing, pressing a key to continue to the next trial. The different TMS SOAs $(0,10$, $20,30,40,50,60,70,80,90,100,110,120,130,140$, and $150 \mathrm{~ms}$ ) and no-TMS trials were randomly interleaved throughout the whole experiment. The different conditions were grouped in 15 task blocks of 17 trials. The order of these blocks was pseudorandomized. Subjects could take a break whenever and however long they wanted between task blocks, but were asked to at least take a break halfway through the session to rest.

In 4 subjects, a separate 'NoTMS' run was added, containing 20 trials. Because of the absence of a TMS pulse in the NoTMS condition during the actual experiment, participants could respond differently due to the expectance of a pulse. Indeed, the average no-TMS performance over all four participants was 0.90 in the separate no-TMS block, while it was 0.78 for these four participants in the no-TMS trials interleaved in the actual experiment. Considering this large difference, we decided not to use no-TMS as baseline, but rather to take a more conservative approach (see section on "Analysis").

Biphasic TMS pulses were administered with a figure-8 coil (MC-B70), over early occipital cortex. The coil handle was oriented laterally to the right, with initial current direction going away from the handle. Target site localization was based initially on phosphene induction. If a TMS-induced phosphene (nearly) overlapped with the visual field location that corresponded to where visual stimuli would later be presented, the coil was fixed. Stimulation intensity was individually calibrated initially at $\sim 10 \%$ above phosphene threshold. When in initial masking trials the participants indicated rarely to subjectively perceive the stimulus orientation, TMS intensity was

\footnotetext{
${ }^{4}$ In the same session an equal number of trials, using the same stimuli, were obtained using a secondary task for a different experiment involving color judgment: this experiment will not be reported here or elsewhere. The original intentions behind this secondary experiment caused our stimuli to be red on half the orientation trials, and blue on the other half of trials. These color differences were balanced between time windows and of no further influence or import.
} 
decreased to the point where participants indicated sometimes to perceive the stimulus, and sometimes not. The average intensity finally used was $58 \%$ machine output (MagPro X100).

Since our results show differences between time windows that were $10 \mathrm{~ms}$ apart, we were prompted to reconfirm whether our experimental setup was accurate in this range. To this end, we performed calibration measurements. A photodiode was applied to the TFT monitor, measuring luminance changes on an oscilloscope. Simultaneously, the TTL pulse leaving the experimental computer's parallel port (which otherwise triggers the TMS machine with negligible delay) was measured. Using this setup, we tested latencies of visual stimulus and TTL pulses presented by the stimulation program also used in the experiment, in several neighboring visual stimulus-TMS triggering pulse SOAs (i.e., time window conditions). By these measurements, we could not confirm if all time windows were not shifted by milliseconds, although a stable monitor response delay should have been offset by a programmed TMS discharge delay. These calibration measurements were rather performed to check whether requested increases in TMS SOA were reliable. Indeed, the measurements confirmed that requested 10-ms increases in SOA between stimulus and TMS pulse were perfectly (accurate to the sub-millisecond range) presented by the experimental setup. These calibration measurements thus showed conclusively that whichever temporal pattern of findings we obtained (see "Results") was not due to technical decisions/limitations.

\section{Analysis}

Since this study was exploratory in nature, we included all ten measured participants in the analyses. This conservatively included also participants where individual masking curves showed limited effects (see Fig. 3). In fact, two of the three subjects, where no multiple-dip pattern of results could be observed (see "Results"), had performance across conditions consistently above no-TMS. Thus, masking did not work at all in these participants. Nonetheless, they were included in all analyses - in acknowledgment of the exploratory nature. Note that this conservative procedure across the board makes any and all effects in the final group results probably weaker than necessary. Since our no-TMS baseline was compromised, probably due to an 'oddball-like' disruptive effect of the unexpected absence of the TMS pulse in randomly interleaved no-TMS trials, we also took a conservative baseline to base our statistical tests on. We took the average (performance or visibility rating) of all trials in the experiment to compare fluctuations around this mean to. This we refer to as the 'all-round mean'. Note that potential TMS effects would already bias this all-round mean downward, making it harder to detect these TMS effects. With 17 conditions, it is not feasible to do statistical correction for multiple comparisons, given TMS effect sizes in general but probably sensitive TMSmasking effect sizes in particular. Thus, we reasoned that taking this conservative allround mean as reference for each individual time window, combined with several conservative analysis decisions mentioned above, would moderately compensate for the multiple comparisons problem. To thus explore TMS-masking effects, we performed pairwise t-test analyses (one-tailed) on each time window separately. The same analysis 
was performed for both objective orientation discrimination performance and subjective visibility ratings.

As presented in the Results section, our data required a follow-up analysis to investigate whether objective masking precedes subjective masking. For this analysis, we looked at individual masking curves and extracted peak masking latencies for these two measures. Peak masking was defined as the lowest performance (or visibility rating) over all SOAs. If peak masking occurred at two SOAs with equal masking, the average of these SOAs was taken as peak masking latency value.

\section{Results}

In this study, we applied single-pulse TMS over all SOAs between 0 and $150 \mathrm{~ms}$ in steps of $10 \mathrm{~ms}$. We measured the effects of TMS pulses on objective visual task performance (an orientation discrimination task) and on subjective orientation visibility rating (on a scale of one to four). Figure 2 shows the resulting data for both measures separately.

\section{Objective discrimination performance}

The upper graph shows the group masking curve of all ten participants, of the objective measure (orientation discrimination task). Whereas classic TMS-masking curves generally display a single broad dip within the classical-masking time window (between 60 and $120 \mathrm{~ms}$, see above), or secondary dips in remote time windows, our data may suggest a different scenario. Orientation discrimination accuracy was decreased in 70 $\mathrm{ms}, 90 \mathrm{~ms}$, and seemingly in $110 \mathrm{~ms}$, in what seem to be three separate dips in performance. In between these SOAs, at $80 \mathrm{~ms}$ and at $100 \mathrm{~ms}$, there seemed to be no masking effects - not a slight decrease of effect, but a real return to baseline. Statistically, the earlier two of these three dips are confirmed to be significant. As explained in the Methods section, we conservatively compared masking effects in individual time windows with the all-round mean, yet did not correct for multiple comparisons. Table 1 reveals the $t$ and $P$ values for these comparisons. Since this was an exploratory study, results are tentative and future research should aim to replicate and confirm these findings in hypothesis-driven experiments. For the moment, in our comparison to all-round mean, our data suggest that there was a behavioral impairment at $70 \mathrm{~ms}(t(9)=2.41 ; P<0.05)$ and at $90 \mathrm{~ms}(t(9)=1.93 ; P<0.05)$. Note that time window $0 \mathrm{~ms}$ closely approached significance, with $(t(9)=1.80 ; P=0.053)$. In light of previous research (Laycock et al. 2007), this very early dip might also reflect behaviorally relevant processing. 
$\begin{array}{cr}\text { If } & \text { different } \\ \text { participants } & \text { have }\end{array}$ individual masking curves around $100 \mathrm{~ms}$, but slightly displaced temporally, a group masking curve with separate dips could result artifactually from the averaging of these individual curves. To rule out this option, we looked into the individual masking curves. Individual curves were noisier than the group curve, which is to be expected with a total of 15 trials per time window per subject, on which a single accuracy ratio is based. In spite of this, we noticed that many individual participants showed at least two of the three dips (decreases in performance at SOA 70,90 , or 110 compared to the SOAs immediately before and after). To allow inspection of these 'dips', we provide all ten individual masking curves (focused on the time windows of interest) in Fig. 3. These include curves of participants in whom masking seemed wholly unsuccessful, but were nonetheless included (see "Methods"). With 15 trials per time window, and taking inter-individual differences into account, these results seem remarkably consistent. For instance, one of the two participants without any 'dips' at the appointed
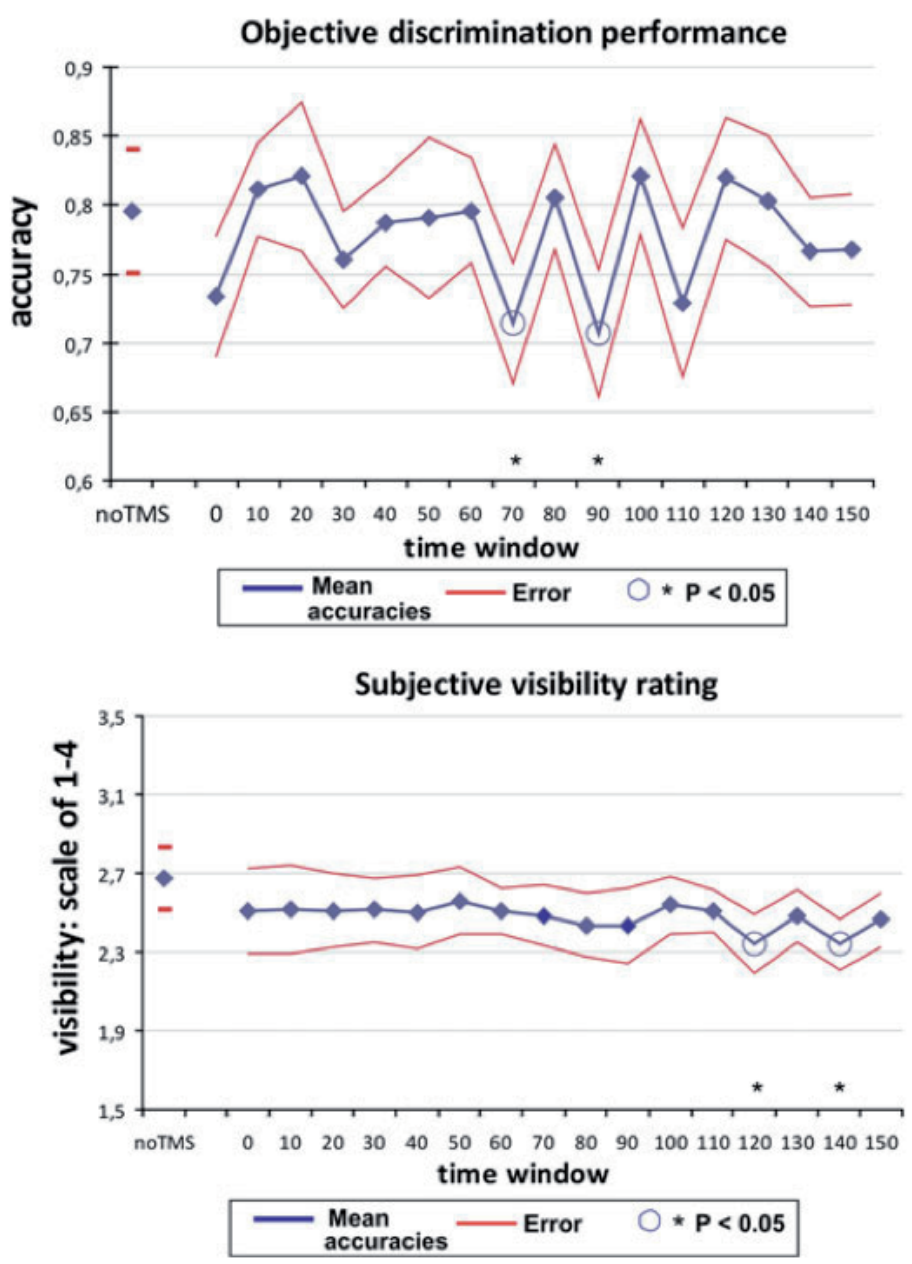

Figure 2: Group masking curves

Upper graph the average performance accuracy across participants is shown in blue. SOAs (time between visual stimulus onset and TMS pulse onset) are on the horizontal axis, proportion correct is on the vertical axis. Red curves shading the performance curve represent the standard error of the mean. Open circles reflect a significant decrease from the all-round mean performance. Lower graph the average visibility rating across participants is shown in blue. SOAs are on the horizontal axis, visibility rating between 1 and 4 is on the vertical axis. 2.5 marks the border between 'seen' and 'not seen'. Red curves again reflect standard error of the mean. Open circles reflect a significant decrease from the all-round mean visibility rating. 
time windows showed two dips at 60 and $80 \mathrm{~ms}$, rather than 70 and $90 \mathrm{~ms}$. Such variability is to be expected, which is why a group curve remains most informative. The individual curves shown in Fig. 3 at any rate do not suggest that the multiple dips in the group map are an averaging artifact based on two or three outliers. Thus, altogether, our data seem to reveal at least two performance dips within the classical-masking time window around $100 \mathrm{~ms}$.
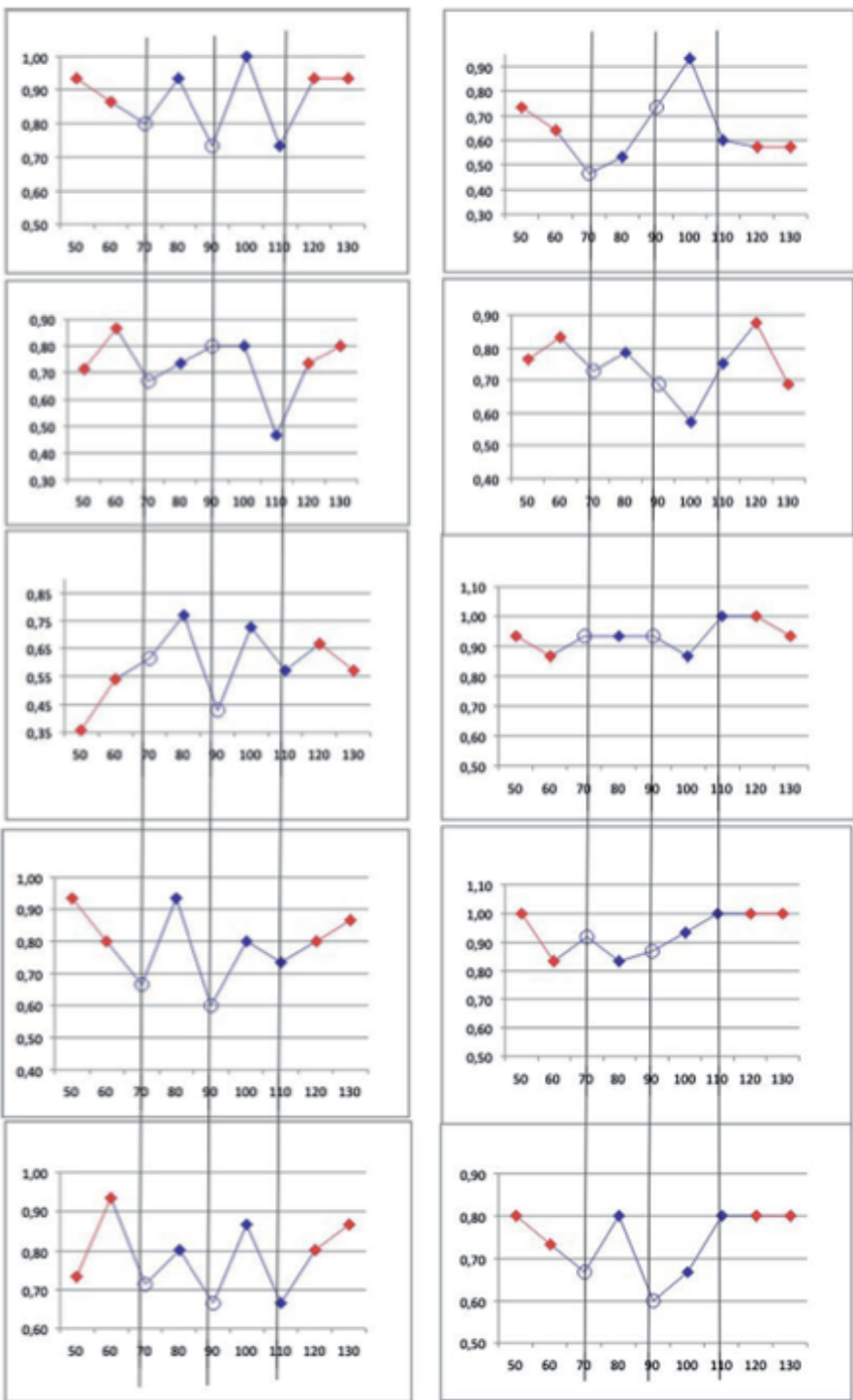

Figure 3:

Individual

masking curves

Focused on the

time windows of

interest (blue

SOAs 70-110),

the individual

performances

over SOAs

(between 50 and

130 shown) are

presented. Note

that proportion

correct is on the

vertical axis, but

that the scale

varies across

participants. The

individual curves

are expectedly

noisier than the

group curve, and

not every

participant

displays the group

pattern in his/her

individual

performance. But

the individual

curves

demonstrate that

the group effect is

not an artifact of

averaging, and

indeed many

participants

display more than one dip. 


\section{Subjective visibility ratings}

The group masking curve on subjective report of visibility did not correspond well to the performance curve (Fig. 2, lower graph). The no-TMS rating was above all TMS pulse time windows, from 0 to $150 \mathrm{~ms}$ SOA. When taking, for instance, the visibility rating of time window $0 \mathrm{~ms}$ as a reference, there is hardly a deviation across time windows. On a scale of $1-4$, in which values 1 and 2 represent 'I didn't see it' and values 3 and 4 represent 'I did see it' (to simplify), the average rating was around 2.5-the border between reporting 'seen' versus 'unseen'. This

shows that our stimuli really were difficult to perceive, as was our intention in establishing a sensitive TMS-masking paradigm. When performing the statistical comparison of individual time windows versus all-round mean, again two time windows reveal a significant TMS effect; 120 and $140 \mathrm{~ms}(t(9)=3.23 ; P<0.05$, and $t(9)=1.84 ; P<0.05$, respectively-see Table 1 for all $t$ and $P$ values). It thus seems to make a difference which measure of vision is adopted, whether (and when) TMSmasking effects seem to occur in a sensitive TMS-masking paradigm. However, we should point out that, although significant, the effects here were exceedingly small. On a scale of $1-4$, the differences between the 120 and $140 \mathrm{~ms}$ time windows to the average visibility rating were only 0.14 and 0.15 , respectively. For comparison, we note that in a different (non-sensitive) TMS-masking study using the same scale (de Graaf et al., 2012), we found a masking effect on subjective visibility rating around 1.5 , which is a full order of magnitude larger. Nonetheless, we acknowledge the statistically significant comparisons. And effect sizes in the objective discrimination task were small as well, 
compared to masking effects in non-sensitive paradigms. This is to be expected when stimulating at lower intensities than is conventional.

This might lead one to suggest that objective vision is disturbed by TMS before subjective vision ( $70-90$ vs. $120-140 \mathrm{~ms}$, respectively). However, we caution that this is not a valid conclusion based on these results alone. An appropriate analysis to address this question specifically is to look at individual peak masking latencies for objective masking, and for subjective masking, and to compare these directly in a within-subject contrast (see "Methods"). This analysis suggests that the average peak masking latencies for objective and subjective masking curves were 97.5 (SD 27.0) $\mathrm{ms}$ and 90.0 (SD 41.4) ms SOA respectively, and not significantly different $(t(9)=0.7 ; P=$ $0.500)$. In our view, it seems that the objective masking curve is interesting and potentially very revealing. The subjective masking curve may not have been sensitive enough to detect these fragile effects.

\section{Discussion}

We here present an exploration of TMS-masking effects at different time windows from visual target stimulus onset, using a sensitive TMS-masking paradigm and two measures of vision. We evaluated whether a sensitive TMS-masking paradigm might yield different masking curves from those revealed previously.

\section{Objective versus subjective measures of visual awareness over time}

Methodologically, we find that the subjective rating measure of visibility was, in the current study, not sensitive enough to obtain convincing masking effects in the classical masking window (60-120 ms, see "Introduction"). This is in line with our experiences using the subjective scale, which generally results in the same pattern of masking effects as objective measures of vision, but is less sensitive chronometrically (de Graaf et al. 2012; next chapter). At any rate, in this study the behavioral masking effects observed within the classical-masking time window were not mirrored by the subjective measure. The fact that TMS affected discrimination performance but not subjective visibility ratings in time-specific windows is, strictly speaking, a dissociation (de Graaf and Sack, 2011). But it is a dissociation opposite to the TMS-induced blindsight that has been reported before (Boyer et al. 2005; Jolij and Lamme 2005). These studies reported that, in the absence of subjective vision, performance was unhampered. In our case, it would seem that with decreased performance, subjective vision was unhampered. But it seems more straightforward to conclude that our measure of subjective vision was less sensitive than the measure of objective vision, than to postulate that objective vision can be disrupted while conscious experience is unchanged. In this regard, it is relevant to keep in mind that the conscious experience of the visual stimuli was always very weak and thus difficult to rate (as is evident from the low visibility rating at baseline). On the other hand, if we take the dips in visibility rating of 120 and $140 \mathrm{~ms}$ seriously, then indeed one might speak of a 'blind-sight'-like dissociation, since conscious experience was affected while behavioral processing was not. In following chapters, we discuss how a TMS-induced blindsight could most likely 
appear in the latter part of the classical masking window. Yet, in the current study this effect size was quite small, so we caution not to stretch interpretations.

\section{TMS-masking effects in a sensitive paradigm}

Our data suggest that, instead of one, there may be at least two dips within the classicalmasking time window surrounding $100 \mathrm{~ms}$. We have already emphasized that our experiment was exploratory in nature, and future research should aim to replicate this finding. But the potential implications upon confirmation are fascinating in our view.

Theoretically, we might consider at least two explanations for neighboring dips. Briefly, it might be that there is not one feedforward sweep and one feedback sweep, but rather multiple recurrent processes (e.g., Lamme and Roelfsema 2000). Communications between regions could repeatedly go back and forth (see, e.g., de Graaf et al. 2009) between hierarchical levels, or within - in the form of horizontal recurrent projections. Foxe and Simpson (2002) argued, conceptually and supported by data, that already at the earliest stages of occipital processing, after visual stimulation, recurrent influences may affect ongoing processing (see Chapter II.5 for more elaborate discussion). It would seem that the very concept of distributed processing suggests that brain regions must interact intensively and repeatedly, if not continuously, to lead to our complex and yet rapid conscious visual experiences. Thus, multiple recurrent loops may explain multiple, chronometrically neighboring, TMS-masking dips within the broad classical time window. Alternatively, the two dips might correspond to arrival in early visual cortex of M-pathways and P-pathways. Magnocellular (M) pathways are faster than parvo- cellular (P) pathways (Bullier 2001), and have been hypothesized to arrive at different times, hence possibly allowing TMS to disturb them at different times (see also Paulus et al. 1999). There is no way to distinguish between these options based on our current data. 



\title{
II. 2
}

\section{Feedforward and Quick Recurrent}

\author{
Processes in Early Visual Cortex
}

\author{
Revealed by TMS?
}

Related publication(s):

De Graaf, TA, Goebel, R, Sack, AT (2012). Feedforward and quick recurrent processes

in early visual cortex revealed by TMS? NeuroImage. 61: 651-59 


\begin{abstract}
Transcranial magnetic stimulation (TMS) can be applied to occipital cortex to abolish (conscious) perception of visual stimuli. TMS research has revealed several time windows of masking relative to visual stimulus onset, most consistently a time window around $100 \mathrm{~ms}$ post-stimulus. However, the exact nature of visual processing in this 'classical' time window, e.g. whether it represents the feedforward processing of the visual information, or rather a feedback projection from higher visual areas, remains unclear. Here, we used TMS to mask in the same participants two types of stimuli of different complexities (orientation Gratings and Faces) over different time windows. Interestingly, the masking functions were not the same for both stimulus types. We found an earlier peak masking latency for orientation stimuli, and a slower recovery for Faces. In a second, follow-up experiment, we superimposed both types of stimuli to create one composite stimulus set. Depending on the instruction, participants could then perform orientation or face discrimination tasks on the exact same stimuli. In addition, for each participant, stimuli were calibrated to equate task difficulties. The peak masking latency was now identical for both tasks, but the masking function revealed again a slower recovery during the face discrimination task, suggesting top-down (recurrent) effects in the second half of the masking function. Hence, rather than this masking window reflecting either feedforward or feedback processing, the early part of what is traditionally considered one masking window may reflect feedforward processing, while the latter part may already reflect recurrent processing. These findings shed new light on recurrent models of vision and related theoretical accounts of visual awareness.
\end{abstract}




\section{Introduction}

Transcranial magnetic stimulation (TMS) can be applied to mask otherwise visible visual stimuli (Kammer, 2007a; Sack, 2006). By interrupting brain processing in early visual cortex directly, in a chronometric design, TMS can reveal crucial points in time for the establishment of a conscious percept (Breitmeyer et al., 2004; Kammer, 2007a, 2007b). This means that TMS indicates when, over time, this specific brain region must remain unperturbed for conscious vision to be established. Classically, such research has resulted in a specific time window for single-pulse TMS masking, around $100 \mathrm{~ms}$ post target stimulus onset (Amassian et al., 1989; de Graaf et al., 2011a, 2011b; Jacobs et al., 2012; Kammer, 2007a; Kammer et al., 2005; Sack et al., 2009). However, earlier (Corthout et al., 1999; Kammer et al., 2003; Laycock et al., 2007; Paulus et al., 1999) and later (Camprodon et al., 2010; Heinen et al., 2005) TMS effects have been reported, adding to mounting evidence for the existence of at least two separate time windows for masking by TMS over early visual cortex (Kammer, 2007b; Koivisto et al., 2010; Silvanto et al., 2005b).

Traditionally, hierarchical models postulated that visual information proceeds upward through a series of hierarchical layers in the visual system (e.g. DeYoe et al., 1994; Felleman et al., 1997). But massive feedback connections exist as well (Maunsell and van Essen, 1983), and recent feedforward/feedback (interactive) models of vision suggest that a recurrent feedback stream of information is necessary to establish detailed or conscious vision (Bullier, 2001; Hochstein and Ahissar, 2002; Lamme and Roelfsema, 2000). Indeed there is concrete evidence that recurrent streams of information from higher-order regions to early visual cortex are required for visual perception (Beckers and Homberg, 1992; Cowey and Walsh, 2000; Pascual-Leone and Walsh, 2001; Ro et al., 2003; Silvanto et al., 2005a, 2005b). These studies, postulating feedforward versus feedback streams of information, nicely match the idea of two TMS masking dips. Only the precise temporal position of these two masking windows is unclear, considering the ambiguity of alternate masking time windows both before and after the classical window. A point of inquiry and indeed speculation has thus been whether the classical masking window of $100 \mathrm{~ms}$ reflects disruption of feedforward or feedback processes (e.g. de Graaf et al., 2011a, 2011b; Kammer, 2007b; Koivisto et al., 2011, see Chapter II.5).

In the current work, we set out to investigate whether the classical masking window around $100 \mathrm{~ms}$ post-stimulus reflects feedforward or recurrent processing, or perhaps even both as recently hypothesized (Koivisto et al., 2011). We reasoned that it might be informative to use different types of stimuli with different complexities of informational content to be masked by TMS in the same participants. For example, if masking around $100 \mathrm{~ms}$ disrupts recurrent processes, the chronometric masking curve for simple stimuli (orientation Gratings) might be different than the masking curve for more complex stimuli (Faces). Possibly, more complex stimuli need more processing time further 'upstream' before being 'fed back', leading to two temporally shifted masking curves. If so, this would indicate very rapid feedback to early visual cortex, already around $100 \mathrm{~ms}$ after stimulus onset. Alternatively, if the classical masking 
window reflects feedforward processing only, the masking curves for simple versus complex stimuli could be identical.

But it is also possible that the masking window of several tens of milliseconds does not neatly reflect either the feedforward or the feedback effect (Chapter II.5). In that case, parts of the masking curve could be identical, such as the onset of masking, while other parts might be shifted, such as the recovery of task performance at longer latencies. This could indicate feedforward and then quick feedback contributions to the single masking curve. In effect, this could mean that both feedforward and feedback processes occur within several tens of milliseconds in early occipital cortex, around 100 ms after visual stimulus onset.

In the current study, we mapped the TMS masking curves between 50 and 130 ms after target stimulus onset. We used two types of stimuli: orientation Gratings and Faces, under the assumption that these are stimuli of increasing complexity (orientation Gratings being most simple and Faces being most complex). We mapped masking curves of both stimulus types using both an objective measure of visual acuity, and subjective reports of stimulus visibility. In a second, follow-up experiment, we more rigorously controlled for task difficulty, and moreover used one composite, superimposed image of Gratings/Faces, rather than two separate sets of stimuli. Note that in this follow-up experiment, the stimulus was thus exactly the same in both the orientation judgment task and the Faces judgment task. Any potential differences in the masking curves for both tasks could therefore be explained as task-induced, top-down effects.

\section{Materials and Methods}

\section{Experiment one}

\section{Participants}

Fourteen participants (four male; age range: 20-42) volunteered for this study. All had normal or corrected-to-normal vision and no history of neuropsychiatric disorders. The experiment was approved by the local medical-ethical committee, written informed consent was obtained before participation. Participants were screened for TMS experimentation safety by an independent medical supervisor, and were compensated with course credit or coupons. Three participants were excluded, because masking in one of both tasks was unsuccessful as indicated by a consistent behavioral performance over $80 \%$ correct in all TMS SOAs, leaving a total of eleven participants with clear masking curves for both tasks in experiment one. 


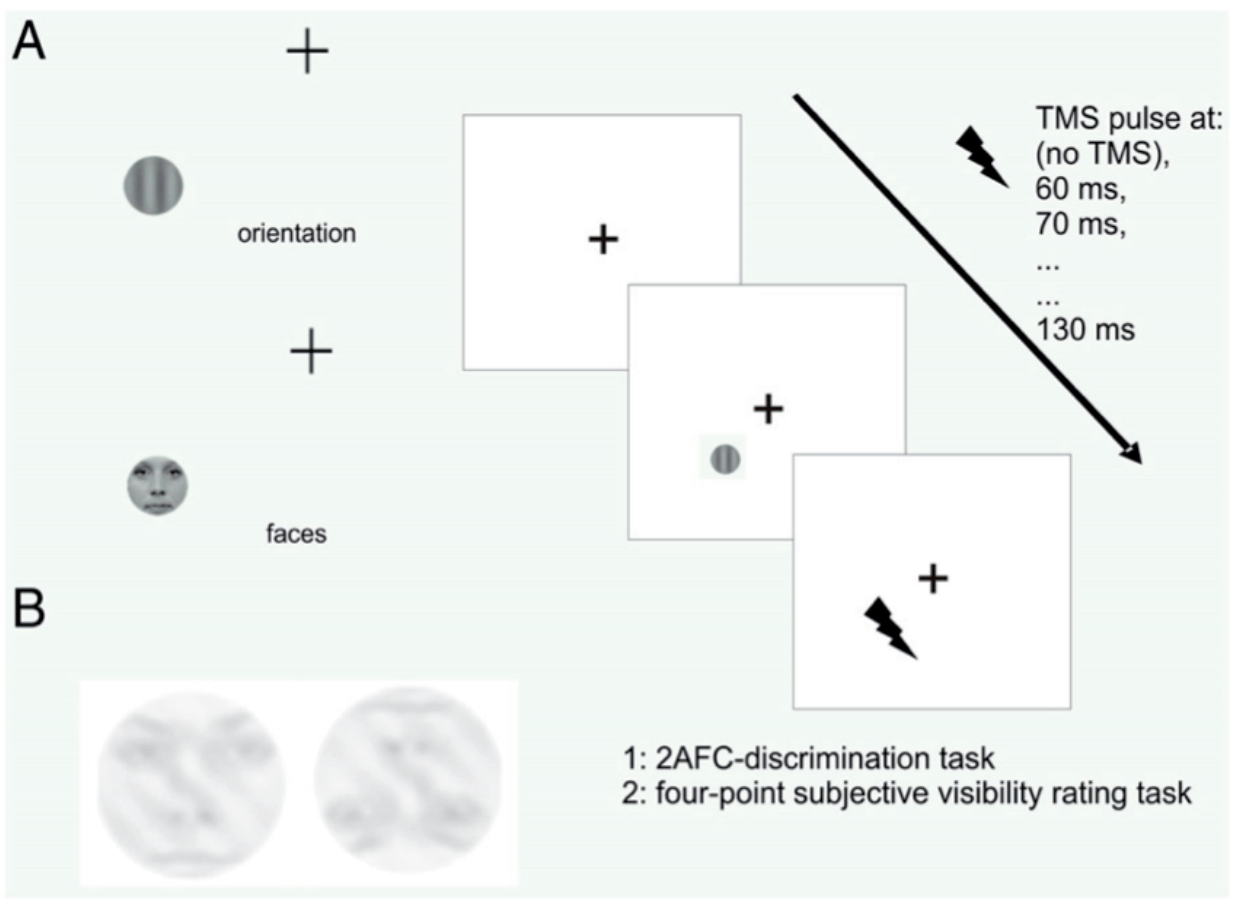

Figure 1. Stimuli and design

A. Orientation Gratings and Faces stimuli were masked by occipital TMS at various time windows. Gratings could have horizontal orientation or vertical orientation. Faces could be upright or inverted. Shown on the right is the design for experiment one. TMS pulses could be applied at visual stimulus - TMS onset asynchronies (time windows) ranging from 60 to $130 \mathrm{~ms}$ in steps of $10 \mathrm{~ms}$. Participants were asked to indicate the orientation of the Grating in Grating task blocks, or the putative inversion of the face in face task blocks. Then they provided a subjective rating of stimulus visibility on a scale of one to four. B. Shown are two of the four stimuli in our follow-up experiment. Shown are an upright face superimposed on a left-tilted Grating, and an inverted face superimposed on a left-tilted Grating. Gratings could also be right-tilted. These four stimuli were used in both a Grating task, where subjects indicated the orientation of the Grating in the stimulus, and a Faces task, where subjects indicated the putative inversion of the face in the stimulus. Thus, the same stimuli were used for both tasks. Other differences with experiment 1 were the measured TMS time windows, and a calibration of the stimuli for individual participants such that both tasks (Gratings and Faces tasks) were equally difficult. 


\section{Stimuli and tasks}

We used two types of stimulus and corresponding tasks, shown in Fig. 1. ${ }^{5}$ Each stimulus was presented for 16.7 milliseconds $(\mathrm{ms})$. Orientation stimuli consisted of greyscale Gratings that could be either predominantly horizontal or vertical in orientation. Due to an error in the stimulus fabrication process the horizontal and vertical Gratings were not identical rotated versions of one Grating. Rather, the horizontal Grating had a hint of a vertical Grating 'shining through'. Since we compared orientation stimuli as a category with Faces stimuli, this issue should not affect the main results and interpretations of the current experiment. On these 'Gratings' stimuli the discrimination task required subjects to indicate with button presses whether the orientation was vertical (left Shift key on a standard keyboard) or horizontal (right Shift key). 'Faces' stimuli consisted of greyscale circular cut-outs of male Faces and female Faces (the sex was of no import for the task). All these Faces were either upright (left Shift key) or inverted (right Shift key). All stimuli were of equal size (circular, see Fig. 1, 1 degree visual angle in diameter), and positioned 4 degrees visual angle diagonally (lower-left) to the fixation cross. Luminance of the stimuli was measured on a TFT monitor (Samsung Syncmaster 931BF) used for stimulus presentation (orientation stimuli: $124 \mathrm{~cd} / \mathrm{m}^{2}$; Faces stimuli on average [ 3 male and 3 female Faces]: $128 \mathrm{~cd} / \mathrm{m}^{2}$ ). Background luminance was always the same: $180 \mathrm{~cd} / \mathrm{m}^{2}$. As we discuss below, luminance values can be important for the masking curve obtained. Note therefore that the orientation and Faces stimuli are nearly perfectly matched.

Task-dependent forced-choice discriminations were made first, and constituted the objective measure of vision. On every trial, participants also indicated stimulus feature visibility as in Chapter II.1. Subjects were given ample time to practice with both discrimination tasks and subjective ratings, until they felt confident and performance on discrimination tasks was well above $80 \%$ without TMS.

\section{Design and TMS parameters}

The experiment consisted of one session. A total of 18 conditions ( 2 stimulus types and 8 time windows + no-TMS) with 12 trials each resulted in 216 included trials per session. Each trial took a minimum of $6 \mathrm{~s}$, with subjects determining the pace by means of button presses (after all responses they would manually begin the next trial). The different TMS SOAs $(60,70,80,90,100,110,120$, and $130 \mathrm{~ms})$ and no-TMS trials were randomly interleaved throughout the whole experiment. The different stimulus types were grouped in task blocks of 12 trials. The order of these blocks was pseudorandomized. Subjects could take a break whenever and however long they wanted to between task blocks, but were asked to at least take a break halfway through the session to rest.

\footnotetext{
5 The first eight participants were measured with a third task involving symbolic (arrow) stimuli. This task proved too difficult in relation to the other two tasks. These data were therefore excluded from analysis and no longer measured in additional participants.
} 
Biphasic pulses were administered with a Magpro X100 stimulator and a figure-8 coil (MC-B70) (Magventure, Denmark), over early occipital cortex. The coil handle was oriented laterally to the right, with initial current direction going away from the handle. Target site localization was based initially on phosphene induction. If a TMS-induced phosphene (nearly) overlapped with the visual field location that corresponded to where visual stimuli would later be presented, the coil was fixed. To check location and define appropriate stimulation intensity, visual stimuli were presented and TMS pulses delivered with an SOA of $90 \mathrm{~ms}$. A block of 20 stimuli was presented at TMS intensity $120 \%$ above phosphene threshold. If at this, or if necessary a corrected, intensity the subject indicated to subjectively sometimes perceive and sometimes not perceive the visual stimulus, the intensity was fixed and kept constant for all the measurements in the actual experiment.

\section{Analysis}

Per stimulus type, and for objective discrimination performance and subjective rating separately, we mapped visual awareness as a function of SOA. The group curves are presented in Fig. 2, showing the averages of individual discrimination accuracies. To define the average peak masking latencies per stimulus type, individual masking curves were mapped as well. The individual peak masking latencies (biggest 'dip' in performance or subjective rating) were identified in these individual curves, and extracted per stimulus type. If in a given subject/stimulus type there were two dips of equal masking magnitude, the average between the two masking latencies was taken, or the first of the two latencies was taken for that individual in that stimulus type/awareness measure. We report results of both methods in the Results section. To evaluate whether peak masking latencies were significantly higher (later) for the complex stimuli (Faces) than the simple stimuli (Gratings), paired-samples t-tests were used (alpha 0.05) on the hypothesis that Gratings would have an earlier peak masking latency. To evaluate other differences between the masking curves, we performed repeated-measures ANOVAs (Huynh-Feldt corrected) and paired samples t-tests as reported.

\section{Experiment two}

Based on the results of experiment one (earlier peak masking latency for Gratings than Faces, overall stronger masking for Faces, and later recovery of the masking function for Faces stimuli), we performed a follow-up experiment. The rationale behind all design choices is elaborated on in the Results section, where we present the results of experiment one.

\section{Participants, stimuli, tasks}

A new subject sample of eight participants was tested. We made four composite stimuli, all of which consisted of superimposed orientation Gratings and Faces. Two of these stimuli are presented in Fig. 1. We arbitrarily selected one female face for the 
composite stimulus. Since the Gratings task in experiment one was ultimately a little too easy, we rotated the Grating stimulus to either be diagonally to the left, or diagonally to the right, at 45 degree angles to vertical, and made the stimulus as a whole more transparent and difficult to perceive. Please see Fig. 1B for the resulting stimuli and compare to Fig. 1A. Thus, the four stimuli in this experiment were: Grating diagonally left/upright Face, Grating diagonally left/inverted Face, Grating diagonally right/upright Face, and Grating diagonally right/inverted Face. Participants only performed one task: the Gratings (orientation judgment) task (diagonally to left: left Shift key, diagonally to the right: right Shift key), or the Faces (inversion judgment) task (upright or inverted, same as in experiment one). Blocks and trial design were the same as in experiment one. Numbers of trials per condition cell were different from experiment one, and varied across subjects. While we measured 12 trials per time window in experiment one, we generally measured 16 trials per time window in experiment two, but due to longer calibration phases for task difficulty in three subjects, we were able to measure 13 trials in one, and 10 trials in two subjects. As we took average values per condition cell per subject, this did not affect our analyses or results.

\section{Procedure, TMS, analysis}

A major difference with experiment one was that we calibrated the relative transparency of the Faces and the Gratings in the composite stimuli on an individual basis to achieve equal task difficulty. Prior to testing, subjects performed a no-TMS block of 20 trials of both tasks on a set of stimuli. If one task or the other was performed better with a percentage difference of more than 3\%, the transparency of the Grating was increased or decreased slightly, and the procedure was repeated. This successfully had the effect that the final stimulus set for each single participant led to equal performance on both tasks in pure no-TMS blocks. Naturally, this one set of stimuli was then used throughout the actual experimental measurements.

The TMS procedures were largely similar to experiment one, with the exception of TMS SOAs measured. In experiment one, we found a peak masking latency difference of only $10 \mathrm{~ms}$, and in a recent unrelated masking study we revealed that TMS SOAs separated by only $10 \mathrm{~ms}$ can have vastly different behavioral results (de Graaf et al., 2011b). Therefore, in the current experiment we increased the temporal resolution even further, in accordance to the guidelines outlined in the aforementioned paper. We zoomed in on the time windows of interest with an unprecedented temporal resolution of $5 \mathrm{~ms}$ steps between SOAs. Thus, we measured the SOAs $50 \mathrm{~ms}, 70 \mathrm{~ms}, 80$ $\mathrm{ms}, 85 \mathrm{~ms}, 90 \mathrm{~ms}, 95 \mathrm{~ms}, 100 \mathrm{~ms}, 110 \mathrm{~ms}$, and $130 \mathrm{~ms}$ - along with interleaved noTMS trials. Note here and in Fig. 3 of the Results section, this means that measured time windows were separated by varying temporal distances.

We performed this follow-up experiment to specifically test the findings of experiment one in a more rigorously controlled setting. Thus, the statistical analyses focused on the effects found in experiment one. Specifically, we zoomed in on the late TMS SOAs to directly compare Gratings and Faces masking - testing for a later recovery (thus, bigger masking effect in late time windows) in the Faces task - even 
though the same stimuli were presented. These analyses were uncorrected paired samples t-tests. We focused on these a priori hypotheses because our subject sample was relatively small. We do however present the masking curves as a whole in Fig. 3, and report the results of statistical analyses analogous to experiment one as well.

\section{Results}

\section{Experiment 1}

For both the objective measure and the subjective measure of vision, the group masking curves are shown in Fig. 2. It becomes clear that masking was very successful, with a strong decrease and subsequent increase in both performance accuracies and visibility ratings over time windows. A repeated-measures ANOVA with factors TASK (Gratings and Faces) and SOA (TMS stimulation onset asynchro- ny, or time window: including no-TMS trials a total of 9 levels) showed a significant effect of TASK $(\mathrm{F}=11.81 ; \mathrm{P}<$ $0.01)$ and SOA $(\mathrm{F}=12.84 ; \mathrm{P}<0.001)$, but no significant interaction, for objective performance. Interestingly, for the subjective measure (stimulus visibility ratings) we found a different pattern: no significant effect of TASK, but again a highly significant effect of SOA $(\mathrm{F}=27.50 ; \mathrm{P}<0.001)$, and moreover a significant interaction between TASK and SOA $(\mathrm{F}=2.30 ; \mathrm{P}<0.05)$. We thus looked further by exploring taskspecifically the effects of TMS at different SOAs.

In a task-specific repeated-measures ANOVA, the TMS time window (SOA) had a significant effect on proportion correct in the objective discrimination task for Gratings $(\mathrm{F}=9.01 ; \mathrm{P}<0.001)$, and Faces $(\mathrm{F}=7.84 ; \mathrm{P}<0.001)$. SOA also had a significant effect on subjective visibility ratings for Gratings $(\mathrm{F}=21.59 ; \mathrm{P}<0.001)$, and Faces $(\mathrm{F}=22.57 ; \mathrm{P}<0.001)$. In spite of the different results from the full ANOVAs for the objective and subjective measures, in general a good correspondence between the objective performance (Fig. 2 left) and the subjective ratings (Fig. 2 right) could be observed. It seems that the objective measure is more time-specific than the subjective measure (the performance masking curves are 'sharper', the visibility curves are a little wider - almost a smoothed version of the performance curves). These observations become clearer when data are normalized for individual participants to baseline (no TMS), and objective and subjective masking curves are put together as in Supplementary Fig. S1. Statistically there was no effect of visual measure (objective versus subjective) when analyzing these normalized data in a repeated-measures ANOVA for Gratings $(\mathrm{F}=2.15 ; \mathrm{P}>0.1)$ or Faces $(\mathrm{F}=0.02 ; \mathrm{P}>0.1)$.

In summary, TMS had disruptive effects on task performance, in a timespecific manner, for both the objective and subjective measures of vision. There was evidence that the stimulus type/task (Gratings or Faces) had an effect on the masking curve obtained. 
A Objective Measure: Performance

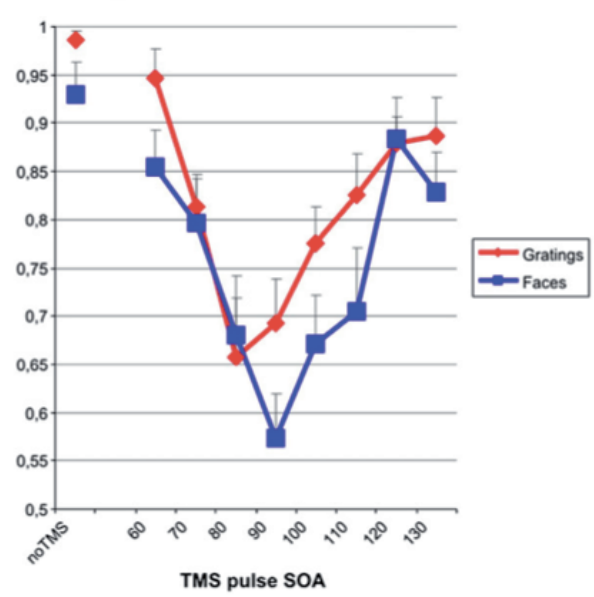

B Subjective Measure: Visibility Rating

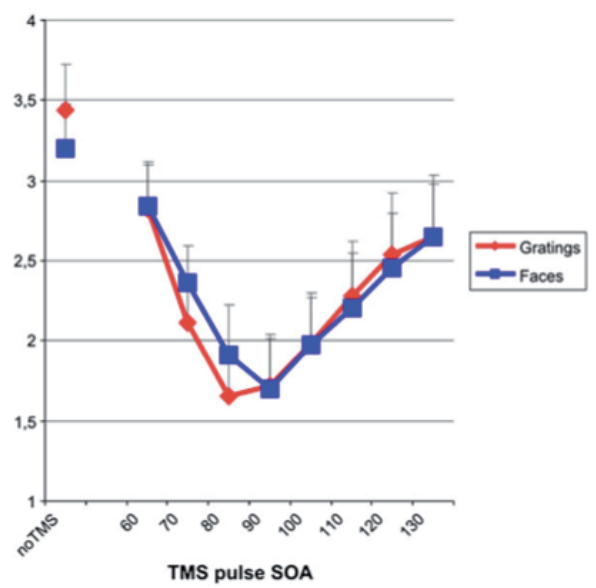

Figure 2: TMS masking curves for Gratings and Faces stimuli/tasks using different stimuli A. On the horizontal axis are the visual stimulus - TMS onset asynchronies (time windows), on the vertical axis is the average proportion correct responses on the forced-choice discrimination task. The average over all subjects is shown for each condition by a marker, error bars reflect standard error of the mean. In red is shown the masking curve for the Gratings stimuli, in blue is shown the masking curve for the Faces stimuli. Statistical analysis confirmed that there was an overall effect of task, with the Faces task being performed less accurately. Other confirmed

observations are a later peak masking latency for Faces than for Gratings, and a slower recovery for Faces than for Gratings. Thus, stimulus/task had an effect on the TMS masking curve. B. Instead of proportion correct, the vertical axis here marks the average subjective stimulus visibility ratings. While masking certainly occurred, and while a different peak masking latency seems mirrored here as well, the curves on the whole appear 'smoothed'. However, as shown in Fig. S1 of the Supplementary material (below), when both the objective and subjective measures of vision are normalized and overlaid, TMS largely seems to have similar effects on both.

\section{Peak masking latency}

What is apparent in Fig. 2 is that the largest masking effect for Gratings occurred at a latency of $80 \mathrm{~ms}$, while for Faces it occurred at a latency of $90 \mathrm{~ms}$. Thus, by eye, it seems that stimulus type had an effect on 'peak masking latency', the SOA where masking was optimal. To test this observation statistically, we extracted for each individual subject the peak masking latency of both stimulus types (Gratings, Faces), to compare individual peak masking latencies between stimulus types across subjects in a paired-samples t-test. Moreover, we used different ways to extract the individual peak masking latencies to validate our finding.

We extracted the SOA per subject at which the proportion correct was at the absolute lowest level (the absolute lowest point in individual masking curves of a given stimulus). If the absolute lowest proportion correct was the same in two SOAs, the first 
SOA at which this masking was obtained was taken as value. The resulting peak masking latencies were $84.55 \mathrm{~ms}$ (standard error of the mean [SEM]: 2.1) for Gratings, and $94.55 \mathrm{~ms}$ (SEM: 3.4) for Faces. The peak masking latency for the Faces stimuli was significantly later (paired-samples t-test, $\mathrm{t}(10)=3.32, \mathrm{P}<0.01$ ) than for Gratings. In a second, alternative, analysis we averaged between SOAs that showed the same absolute lowest level of performance (instead of taking the first SOA), and found again a significant difference (Gratings: $89.09 \mathrm{~ms}$ [3.4], Faces: 96.55ms [3.5], t(10) $=2.19, \mathrm{P}<$ $0.05)$ with, again, the peak masking latency for Faces stimuli being significantly later than for Gratings. These results seem to suggest that our two different stimulus types had different peak masking latencies.

\section{Recovery of masking function}

Aside from this later peak masking latency for Faces as compared to Gratings, Fig. 2 also shows a later recovery of the masking curve for Faces than for Gratings. In other words, at later masking windows, Faces stimuli were still masked quite strongly, while Gratings stimuli were less easily or strongly masked by the same TMS intensity in the same subjects. This later recovery might or might not be related to the earlier peak masking latency discussed above. Incidentally, while in Fig. 2B the later peak masking latency seems reflected in the subjective measure of vision as well, the late recovery cannot be observed in the subjective ratings.

We explored this late-recovery observation using post-hoc statistics. Conservative fully-corrected (bonferroni: 16 comparisons) two-tailed paired-samples ttests, comparing objective task performance (accuracy) at different TMS SOAs versus no-TMS baseline showed that TMS statistically significantly masked the visual stimuli in the Gratings task at SOAs $70 \mathrm{~ms}(\mathrm{t}(10)=4.93 ; \mathrm{P}<0.01), 80 \mathrm{~ms}(\mathrm{t}(10)=5.60 ; \mathrm{P}<$ $0.01)$, 90ms $(\mathrm{t}(10)=5.79 ; \mathrm{P}<0.01), 100 \mathrm{~ms}(\mathrm{t}(10)=5.28 ; \mathrm{P}<0.01)$, and in the Faces task at SOAs $80 \mathrm{~ms}(\mathrm{t}(10)=3.99 ; \mathrm{P}<0.05), 90 \mathrm{~ms}(\mathrm{t}(10)=7.17 ; \mathrm{P}<0.001), 100 \mathrm{~ms}(\mathrm{t}(10)=$ 4.82; $\mathrm{P}<0.05)$, and also $110 \mathrm{~ms}(\mathrm{t}(10)=4.50 ; \mathrm{P}<0.05)$. In other words, we see the earlier masking latencies for Gratings reflected in the fact that Gratings were significantly masked at $70 \mathrm{~ms}$, but Faces not yet. And we see the later recovery for Faces reflected in the fact that Faces were still significantly masked at $110 \mathrm{~ms}$, but Gratings not anymore. However, no-TMS is a tricky baseline, as discussed below, so we also performed post-hoc paired-samples t-tests (one-tailed, uncorrected) between Gratings and Faces tasks for the different TMS SOAs, to more directly explore at which SOAs a TMS pulse led to lower performance on Faces than on Gratings tasks. In this analysis, we found a difference at SOAs $60 \mathrm{~ms}(\mathrm{t}(10)=2.98 ; \mathrm{P}<0.01), 90 \mathrm{~ms}(\mathrm{t}(10)=$ 2.58; $\mathrm{P}<0.05)$, and $100 \mathrm{~ms}(\mathrm{t}(10)=1.90 ; \mathrm{P}<0.05)$, and a trend at $110 \mathrm{~ms}(\mathrm{t}(10)=$ $1.70 ; \mathrm{P}=0.06)$. Note that in this liberal, explorative analysis, the no-TMS difference would (just) reach significance as well, which points out the need to consider task difficulty in interpreting these results (see below), and further supports the need for a second experiment to relate to the weaker of the observations above.

In summary, in this experiment we compared the traditional TMS masking curves that are obtained when masking orientation Gratings versus Faces. We revealed interesting differences, including an overall TASK effect, an earlier peak masking 
latency for Gratings as compared to Faces, an interaction between TASK and TMS SOA specifically in the subjective ratings of stimulus visibility, and a potential later recovery of the masking function for Faces as compared to Gratings, specifically in the objective forced-choice discrimination task.

However, any or all of these theoretically and methodologically interesting differences could be attributed to either of three of the following possible causes in experiment one: first, a potential difference in task difficulty. The Gratings task seemed to be easier in baseline (no TMS) than the Faces task. As can be seen in the group curves of Fig. 2, baseline performance did not seem to be equal across all conditions in our sample of subjects (in contrast to initial pilot measurements in different participants), which might confound the results. We must consider this point even though the difference in baseline performance was not statistically significant in our initial two-tailed uncorrected paired samples t-test (no-TMS performance: $t(10)=1.82$; $\mathrm{P}=0.1$ ) and closer inspection revealed that one participant specifically had a rather low Faces baseline (removing this subject did not qualitatively change the pattern of results reported over SOAs or between tasks). The baseline difficulty was actually quite difficult to ascertain in experiment one, since many subjects were on ceiling performance in no-TMS baseline. Thus, is the overall objective TASK effect (Faces task over all SOAs was performed worse) due to a baseline task difficulty difference, or to something else? Secondly, the stimuli themselves were physically different. Although Gratings and Faces stimuli were not appreciatively different in luminance (which has an effect on peak masking latency (Kammer, 2007a), any number of image properties were different. It is impossible to state whether orientation Gratings as an informational category are masked differently from Faces stimuli as an informational category, based on two physically different stimuli, since any lower-level visual feature differences than category might confound this interpretation. Thirdly, the tasks were different. In the Gratings task, subjects were actively paying attention to the orientation information in the stimulus. In the Faces task, subjects were actively seeking out facial features. Any differences in masking curves between Gratings and Faces might be due to top-down influences stemming from this visual search bias. Considering that the conventional conception of a 'feedforward sweep' seems to be an automatic parallel process (Lamme and Roelfsema, 2000), a top-down effect on the traditional masking curve would suggest to us that this masking curve might not reflect only a disruption of the feedforward sweep.

Therefore, while interesting both theoretically and methodologically, our revealed differences in masking characteristics between Gratings and Faces could become more meaningful still if combined with our follow-up experiment.

\section{Experiment 2}

In experiment 2 we again set out to compare the TMS masking curves of orientation Gratings, versus Faces, but we sought to complement our initial measurements in the following ways: 1) we controlled more rigorously for difficulty, equating in individual 
participants the difficulty of both tasks below ceiling level in a calibration phase before the actual measurements, 2) we used composite images of orientation Gratings and Faces, so that between the Gratings and Faces tasks, only the task differed while the visual stimulus was exactly the same. Note that if we still found differences between both masking curves, these could be attributable to top-down influences on visual processing, and 3) we 'zoomed in' on the time frame of interest by measuring with an unprecedented high temporal resolution of $5 \mathrm{~ms}$ steps.

Fig. 3 shows the group masking curves for both tasks in experiment 2 for the objective visual discrimination tasks. Three findings seem to stand out. Firstly, there is no clear indication of an earlier peak masking latency for the Gratings task as compared to the Faces task. Secondly, there seems to still be a later recovery of the masking curve for the Faces task as compared to the Gratings task. Thirdly, the masking curves are rather 'peaky' between the $5 \mathrm{~ms}$ separated SOAs. We consider this last observation further in the Discussion below. Note also that baseline performance in both tasks seemed similar and well below ceiling. Statistically, this was borne out: no significant difference between both no-TMS conditions for the two tasks $(t(7)=-0.41 ; \mathrm{P}>0.1)$.

We performed the same full repeated-measures ANOVA of TASK $\times$ SOA we performed for experiment one, even though our subject sample of eight participants was rather small for this analysis. In contrast to experiment one, the main effect of TASK did not reach significance $(\mathrm{F}=3.130, \mathrm{P}>0.1)$, although there was a significant effect of SOA $(\mathrm{F}=2.892, \mathrm{P}<0.01)$. Since, based on experiment one, we had a priori hypotheses about the peak masking latency and the recovery of the masking curves, we analyzed these aspects of the dataset further.

The peak masking latencies were extracted similarly as in experiment one (here we now report the method of extracting the individual peak masking latency which was the first SOA at which the absolute lowest performance was obtained). The peak masking latencies were not significantly different (Gratings task average $88.9 \mathrm{~ms}$ [SEM 6.9] Faces task average $86.9 \mathrm{~ms}[3.1], \mathrm{t}(7)=0.22 ; \mathrm{P}>0.1)$. Given these values, this null finding does not seem to be a matter of statistical power (see chapter III.2).

The later recovery during the Faces task as compared to the Gratings task, in spite of implementing the exact same visual stimuli, does seem preserved. Statistically, paired-samples t-tests show that for the Gratings task, performance was significantly degraded from baseline (no-TMS) at the SOAs of 90ms $(\mathrm{t}(7)=2.14 ; \mathrm{P}<0.05)$ and $95 \mathrm{~ms}$ $(\mathrm{t}(7)=2.19 ; \mathrm{P}<0.05)$. For the Faces task, performance was significantly degraded from baseline (no-TMS) at the SOAs of $50 \mathrm{~ms}(\mathrm{t}(7)=2.69 ; \mathrm{P}<0.05), 90 \mathrm{~ms}(\mathrm{t}(7)=2.28 ; \mathrm{P}<$ $0.05), 95 \mathrm{~ms}(\mathrm{t}(7)=2.48 ; \mathrm{P}<0.05)$, with additionally marginally significant masking at $100 \mathrm{~ms}(\mathrm{t}(7)=1.46 ; \mathrm{P}<0.1)$, and statistically significant masking at $110 \mathrm{~ms}(\mathrm{t}(7)=$ 2.46; $\mathrm{P}<0.05)$. Thus, TMS pulses over occipital cortex disrupted visual discrimination performance during the Faces task at later time points as compared to performance during the orientation Gratings task, even though the same stimuli were used, task difficulty was identical, and the same participants were measured.

However, no-TMS trials are a tricky baseline given the absence of a TMS pulse which can have unexpected, potentially oddball-like effects on a given trial if the visual stimulus is hard to perceive at baseline, as we recently discussed (de Graaf et al., $2011 \mathrm{~b}$; previous chapter). Therefore, we directly compared the performance on the 


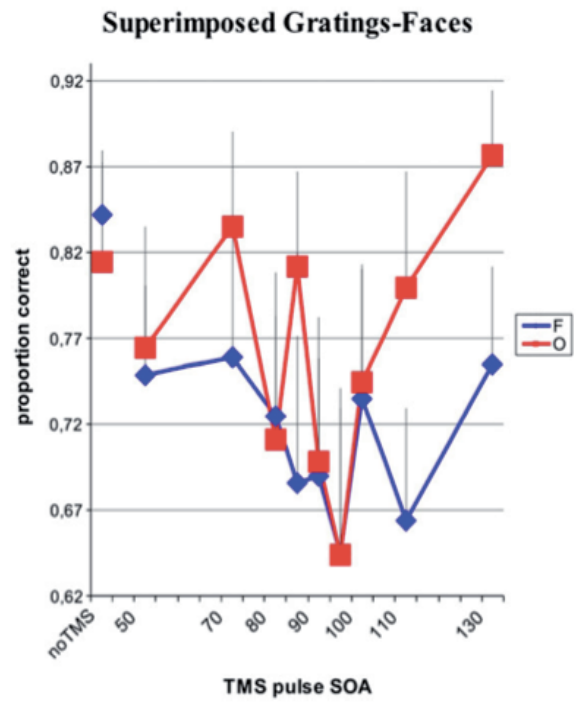

Figure 3: TMS masking curves for the Gratings and Faces tasks using identical stimuli. As in Fig. 2A, the vertical axis reflects proportion correct on the objective forced-choice discrimination tasks; red for the Gratings task and blue for the Faces task. The horizontal axis again reflects TMS time windows, but note that not the same time windows were measured (see Methods, note here that markers point out the time windows actually measured). Three findings stand out. First, there is no difference in peak mask- ing latency. This suggests that the stimulus itself, or task difficulty, rather than top- down task-induced bias, caused the difference in peak masking latency in experiment one. Second, there is still a later recovery for the Faces task. This can only be explained by a top-down task-related influence. Third, in the Gratings task, the TMS curve seems to go back to baseline at $85 \mathrm{~ms}$ SOA, after which masking again occurs. Thus, there are two 'dips' in the curve, which seems to replicate an earlier preliminary observation published in de Graaf et al. (2011b).

Gratings task and the Faces task per TMS SOA. Note that in this comparison, everything was exactly the same between task conditions: same TMS pulse timing, stimuli, and participants. Only the top-down attentional bias and demands related to the performed task differed. In this analysis, we found again that masking was stronger in later TMS SOAs in the Faces task as compared to the Gratings task: performance was significantly lower for the Faces task in SOAs $110 \mathrm{~ms}(\mathrm{t}(7)=2.11 ; \mathrm{P}<0.05)$, and 130 $\mathrm{ms}(\mathrm{t}(7)=2.89 ; \mathrm{P}<0.05)$.

Masking in the Faces task was also significantly stronger at SOA $85 \mathrm{~ms}(\mathrm{t}(7)=$ 2.69; $\mathrm{P}<0.05)$, which was due to the short return to baseline in the Gratings task at this SOA that can be observed in Fig. 2. This return to baseline might be mere noise, were it not for the fact that we observed such a return to baseline around that SOA in a previous study (de Graaf et al., 2011b; previous chapter).

In summary, while in experiment one we found a later peak masking latency and slower recovery for Faces stimuli, in experiment two only the late recovery is replicated, after controlling for task difficulty and physical stimulus characteristics. 
Thus, the peak masking latency difference in experiment one seems dependent on the actual stimulus used or an imperfect control of task difficulty, while the recovery difference may be a top-down effect.

\section{Discussion}

We here reported two experiments that compared the TMS masking functions for different stimuli and tasks. In experiment one, we found that Faces were most strongly masked at a later latency than Gratings, in both objective discrimination tasks and a subjective stimulus visibility rating task. Also, the objective Faces task was performed worse across SOAs, and it seemed as if Faces could be masked at later time points than Gratings. In a follow-up experiment, we masked composite images of Faces and Gratings, on which on a given trial only the Gratings task or the Faces task was performed. Moreover, these two tasks on these stimuli were calibrated to be equally difficult in every single participant. Any potential differences between masking curves would solely be due to the task that was performed, and thus to a top-down attentional/task bias that might have acted on stimulus processing and/or the TMS disruption thereof. We reasoned that the combination of TMS masking information using different stimuli and different tasks would be valuable methodologically, and moreover could illuminate the role of feedforward and/or feedback processes in the traditional TMS masking window. We discuss methodological and theoretical implications of our findings in turn.

\section{Methodological considerations}

Since pioneering work by Amassian et al. (1989), the mapping of masking curves over time has been a most popular research question. It is indeed quite striking that a single TMS pulse in a critical time window, after the onset and offset of a visual target stimulus, can completely abolish that stimulus from conscious perception. But it is exactly because this effect is so meaningful and informative that several outstanding questions should be addressed. While TMS-induced masking has been applied many times before to study vision in the human brain, in contrast to visual masking methods, TMS masking has not yet been rigorously mapped out in terms of what different parameters or dependent variables mean for the masking effects. For instance, some studies have looked at the effect of TMS intensity (Beckers and Homberg, 1991; de Graaf et al., 2011a; Kammer et al., 2005), or coil parameters (Kammer et al., 2007), but most TMS masking studies have investigated the effect of TMS pulse timing, or different SOAs (see Kammer, 2007a, 2007b, and Chapter III.5 for reviews). Few studies, however, have looked at the effect of using different stimulus categories, and to our knowledge no study to date has looked at the effect of using different tasks on the exact same stimulus.

Our findings suggest that stimulus types with different informational contents are processed differentially in early visual cortex, around $100 \mathrm{~ms}$ after stimulus onset, as evidenced by differing TMS masking curves. But it is important to consider how our stimuli were different. Concretely, the Gratings and Faces stimuli differed in 'high- 
level' informational content, in that one stimulus was an orientation Grating, and the other was a Face. But since the stimuli were different, they must have physical differences on a more basic level as well. As far as we know, luminance of visual input affects retino-cortical transmission times. The optimal latency for TMS masking might thus depend on stimulus luminance. Masur et al. (1993) noticed this in two participants, without statistical analyses. In a more systematic approach, Miller et al. (1996) and recently Kammer et al. (2005) showed systematically that the peak masking latency for brighter visual input is shorter; TMS pulses are needed earlier to obtain optimal masking. The modulations in luminance were considerable in this work (Kammer et al., 2005; Miller et al., 1996). Luminance differences are, however, unlikely to explain our own results here (see Methods). Previous work comparing peak masking latencies found no differences between stimuli. One previous attempt compared letters and orientation Gratings, but found no differences in peak masking latency (Amassian et al., 1993). But this study had a lower temporal resolution and used a circular coil. Additionally, one study compared chromatic and achromatic stimuli, but although they found interesting differences between the two groups of stimuli on SOAs in an earlier time window, which we did not measure, the peak masking latency in the classical time window was the same for both (90 ms in that study) (Paulus et al., 1999). Recently, Koivisto et al. (2011) found different maskings of orientation and symbol stimuli, but in separate groups of subjects.

A third methodological variable to consider is the use of objective versus subjective measures of vision. Although performance accuracies (our objective measure of visual processing) showed chronometrically narrower masking effects, while visibility ratings (our subjective measure) revealed seemingly more gradual and undefined masking curves, the pattern of results did not differ significantly. In a direct comparison using normalized data, there was no effect of 'measure used'. Thus, while performance may be a more sensitive measure if it comes to defining the optimal SOA for masking, we here did not observe a clear dissociation of results between the objective and subjective measures. This is in line with our previous experiences (de Graaf et al., 2011a) but seems to stand in contrast to some earlier reports of dissociations (see Koivisto et al., 2011). Recent TMS masking studies have begun to use more than one measure of visual processing (Boyer et al., 2005; Jolij and Lamme, 2005; Koivisto et al., 2010, 2011; Ro et al., 2004). However, our experience that the subjective and objective measures of vision show largely the same responses to TMS over SOAs does not directly contradict most of these studies. For example, Boyer et al. (2005) and Koivisto et al. (2010) selected trials in which subjective ratings were low, and then looked at objective performance in different conditions. This approach is paradigmatically so different that it complements, rather than contradicts, our repeated finding that TMS affects objective and subjective vision in a largely similar way.

\section{Neurobiological considerations}

Our findings are relevant theoretically; as well as methodologically. As outlined in the Introduction, recent models of conscious vision postulate that early visual cortex is important for perception in two stages: one fast feedforward sweep passes V1, and later 
feedback inputs occur (e.g. Lamme and Roelfsema, 2000). The feedback stream has been suggested to be important for conscious perception (Lamme, 2006; Ro et al., 2003; Silvanto et al., 2005a). If we consider that the $100 \mathrm{~ms}$ masking window may reflect the feedback stream, our data do not seem fully in line with such a notion. Not only subjective vision, but both objective and subjective vision, thus visual processing as a whole, was disrupted, although we did not look at objective performance on trials with low subjective vision only (Boyer et al., 2005; Koivisto et al., 2010). Koivisto et al. (2010) showed with motion stimuli that the second TMS masking window was relevant for unconscious vision as well as conscious vision. Thus, it is relevant to note that there are alternative recurrent models, such as the reverse hierarchy theory (Hochstein and Ahissar, 2002). This model does not ascribe a 'consciousness'-role to the feedback stream, but rather a function of filling in the details, or 'vision with scrutiny', where the feedforward stream of visual information sets the scene, or 'vision at a glance' (Ahissar et al., 2009; Hochstein and Ahissar, 2002). See also Bullier (2001).

We cannot decide between these models on the basis of our data. Moreover, these considerations assume that the traditional masking window is either the feedforward window, or a feedback window. While there seems to be some evidence that some visual input arrives earlier than the classical time window around $100 \mathrm{~ms}$, starting around $40 \mathrm{~ms}$, from research with monkeys (Maunsell and Gibson, 1992; Nowak et al., 1995; Raiguel et al., 1989; Schmolesky et al., 1998) and while a separate TMS masking window around 30-40 ms has been reported in some studies (Corthout et al., 1999; Kammer et al., 2003; Paulus et al., 1999), these reports are not consistent (see Kammer, 2007b). And in contrast to these indications, it seems that information in human visual cortex does not really arrive until some $60 \mathrm{~ms}$ after stimulus onset, reflecting mean retino-cortical transmission time, as evidenced by EEG and MEG responses to visual inputs (e.g. Baseler and Sutter, 1997; Foxe and Simpson, 2002; Vanni et al., 1997). This seemingly leads to an impasse: does the traditional masking window reflect the feedforward, or the feedback stage?

Perhaps this question may be misguided. We recently noted that within the classical masking window, there may be more masking dips than one, and thus this broad masking window might reflect something more complicated than a single dip (de Graaf et al., 2011b; previous chapter). We acknowledged in that study that the findings were preliminary and needed replication. It is thus noteworthy that in the current study, in the follow-up experiment, we see again qualitatively a masking dip in the Gratings task at SOA 80 , that goes back to baseline at SOA 85 , only to turn back down to a second masking dip. This is still not a definite confirmation of the existence of multiple masking dips per se, but it does nicely coincide with our earlier report. In that study, we also used orientation stimuli, which were difficult to perceive, with relatively low TMS intensities and a high temporal resolution. That we did not see two masking dips in experiment one of the current report, makes sense: the stimuli were more salient and required higher TMS intensities to be masked, and temporal resolution was lower. In order words, experiment two of the current study constituted a 'sensitive masking paradigm' as outlined in de Graaf et al. (2011b), while experiment one did not.

Both this finding of closely neighboring masking dips, and the other pattern of findings in the current experiments, may fit with a new model of the traditional masking 
window recently proposed. Koivisto et al. (2011) noted that converging evidence from TMS and MEG research supports very early local recurrent processes after the initial arrival of visual input in early visual cortex (Boehler et al., 2008; Foxe and Simpson, 2002). Recurrent processing might start only a few tens of milliseconds after the initial feedforward sweep. It thus seems possible that the wide traditional masking window reflects both feedforward and recurrent processes. Specifically, the first tens of milliseconds of the traditionally wide masking window around $100 \mathrm{~ms}$ post-stimulus onset might reflect a feedforward process, while TMS only slightly after this could be disrupting early recurrent processing (Koivisto et al., 2011). Our findings match this suggestion. ${ }^{6}$

A Gratings - normalized to baseline

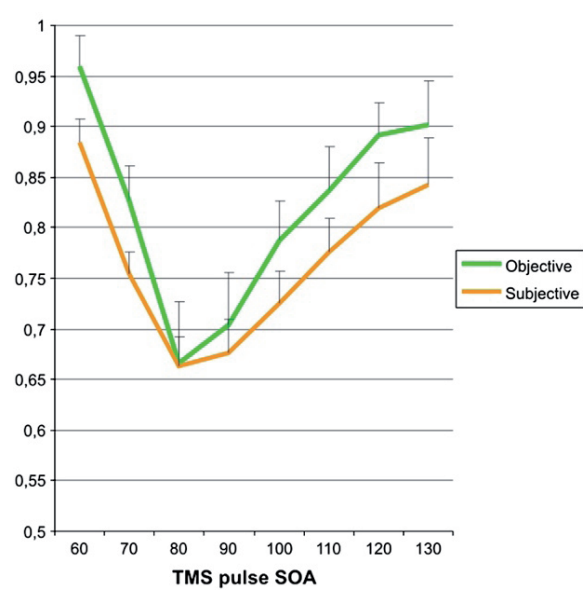

B Faces - normalized to baseline

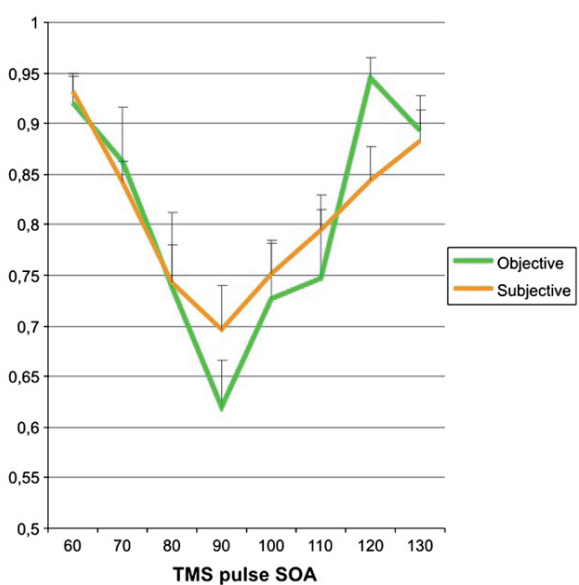

Figure S1: normalized results for both measures of visual processing

The earlier peak masking latency for Gratings versus Faces was found when using different stimuli, but not when using the same composite stimulus. The peak masking latency could reflect still the feedforward phase of processing in early visual cortex, which intuitively should not be affected by a top-down attentional/ task bias (experiment two) but could be affected by physical characteristics of visual input (experiment one). In contrast, a top-down task bias, and/or a stimulus category effect, might affect recurrent processing. Orientation stimuli are processed in the earliest regions (e.g. Hubel and Wiesel, 1968), while Faces are likely processed in the fusiform face area, higher up in the visual hierarchy (e.g. Kanwisher and Yovel, 2006). Thus, it stands to reason that 'feedback about' more complex stimuli would take longer to travel back to the same early visual cortex region that we stimulated with TMS. Or more

\footnotetext{
${ }^{6}$ Note, however, that the implementation of this hypothesis subtly differs from the cited work by Koivisto et al. (2011). These authors focus on the possible link between these early recurrent processes and subjective awareness. We rather focus on the link between these early recurrent processes and task performance, in relation to stimulus category/task bias effects.
} 
complex local recurrent processing is required for the more complex stimuli that were faces. Thus, in experiment two, local or top-down recurrent processes could be responsible for any differences between the Gratings and Faces masking curves, and indeed we found a later recovery of the masking curve for Faces as compared to Gratings. The fact that, of the various differences between both masking curves found in experiment one, only the late 'recovery-effect' was replicated in experiment two, seems in line with the hypothesis that the second half of the traditional masking function around $100 \mathrm{~ms}$ already reflects recurrent processes. 



\section{II.3}

\section{On the Masking Effects of Occipital}

TMS with Varying Intensity when

Applied Before and After Visual Stimuli

(I)

Related publication(s):

De Graaf, TA, Cornelsen, S,* Jacobs, C, Sack, AT (2011). TMS effects on subjective and objective measures of vision: Stimulation intensity and pre- versus post-stimulus masking. Consciousness and Cognition. 20: 1244-1255

\section{* Sonja Cornelsen}

1) Eberhard Karls Universität Tübingen, Psychologisches Institut, Tübingen, Germany 


\begin{abstract}
Transcranial magnetic stimulation (TMS) can be used to mask visual stimuli, disrupting visual task performance or preventing visual awareness. While TMS masking studies generally fix stimulation intensity, we hypothesized that varying the intensity of TMS pulses in a masking paradigm might inform several ongoing debates concerning TMS disruption of vision as measured subjectively versus objectively, and pre-stimulus (forward) versus post-stimulus (backward) TMS masking. We here show that both pre-stimulus TMS pulses and poststimulus TMS pulses could strongly mask visual stimuli. We found no dissociations between TMS effects on the subjective and objective measures of vision for any masking window or intensity, ruling out the option that TMS intensity levels determine whether dissociations between subjective and objective vision are obtained. For the poststimulus time window particularly, we suggest that these data provide new constraints for (e.g. recurrent) models of vision and visual awareness. Finally, our data are in line with the idea that pre-stimulus masking operates differently from conventional post-stimulus masking.
\end{abstract}




\section{Introduction}

TMS masking has been applied repeatedly to investigate how the human brain processes visual information. Such studies have revolved strongly around a temporal question: in reference to the onset of a briefly presented visual stimulus, when do occipital TMS pulses disrupt vision? These chronometric studies have consistently revealed a TMS masking effect around 90- $100 \mathrm{~ms}$ after visual target stimulus onset (Amassian et al., 1989; Beckers \& Homberg, 1991; Christensen, Kristiansen, Rowe, \& Nielsen, 2008; Corthout, Uttl, Walsh, Hallett, \& Cowey, 1999; de Graaf, Herring, \& Sack, 2011; Kammer, 2007a; Sack, van der Mark, Schuhmann, Schwarzbach, \& Goebel, 2009).

Interestingly, some additional time windows in which TMS pulses can interfere with vision have been reported (see Chapters II.1,2,5). One non-classical time we investigate here is the 'pre-stimulus time window' (Corthout, Hallett, \& Cowey, 2003; Laycock, Crewther, Fitz- gerald, \& Crewther, 2007) in which TMS pulses are applied prior to the onset of a visual target stimulus. This pre-stimulus time window is interesting, but difficult to interpret, since TMS pulses could have various non-neural effects that interfere with subsequent vision, such as eye blinks or attentional (alerting) effects. If the pre-stimulus masking effect is genuine, and neural in nature, it is very informative, and likely to be fundamentally different from a post-stimulus masking effect. The existence and possibility of TMS masking in a pre-stimulus masking window is therefore one major question of interest.

Secondly, recently researchers have wondered whether TMS can affect 'conscious vision' while leaving 'unconscious visual processes' largely intact. If so, this would have profound implications for the study of human conscious experience. Boyer, Harrison, and Ro (2005) suggested that TMS can induce 'blindsight' in healthy volunteers, disrupting conscious experience of a stimulus without strongly reducing performance on a forced-choice discrimination task. This above-chance performance without conscious vision was thus suggested to be 'unconscious', and resistant to the TMS manipulation (see also Christensen et al., 2008; Jolij \& Lamme, 2005; Ro, Shelton, Lee, \& Chang, 2004).

Boyer et al. (2005) suggested that the blindsight-like performance was mediated by subcortical pathways. But such data are also interesting in light of recurrent models of human vision (Ahissar, Nahum, Nelken, \& Hochstein, 2009; Bullier, 2001; Di Lollo, Enns, \& Rensink, 2000; Lamme \& Roelfsema, 2000; Ro, Breitmeyer, Burton, Singhal, \& Lane, 2003; Silvanto, Cowey, Lavie, \& Walsh, 2005a). A selective disruption of 'conscious vision' would also be in line with one feedforwardfeedback model of conscious vision, in which a fast feedforward sweep is sufficient for simple task performances, while later feedback activity is responsible for visual awareness of the input (Lamme, 2001, 2006). In such a model, the inconsistently reported earlier TMS masking windows around $30 \mathrm{~ms}$ could constitute the feedforward sweep, while the classical masking effect around $100 \mathrm{~ms}$ post-stimulus might reflect disruption of the feedback activity.

However, the findings in Boyer et al. (2005) have been contested (e.g. Koivisto, Mantyla, \& Silvanto, 2010), and in fact, most TMS masking studies do find 
masking of visual performance around 100 ms (Amassian et al., 1989; Breitmeyer \& Ogmen, 2004; Camprodon et al., 2010; Corthout et al., 1999; Corthout et al., 2003; de Graaf et al., 2011; Heinen et al., 2005; Koivisto et al., 2010): indeed, TMS-induced behavioral impairment constituted the starting point of the entire TMS masking enterprise. Indeed, in our own efforts we could not find consciousness-specific suppression (see previous chapter). Therefore, when considering recurrent models of vision in the context of TMS masking effects, it is important to note that some recurrent models of vision do not propose a 'consciousness-role' for the feedback activity

A

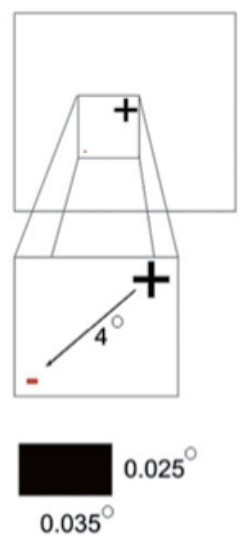

B

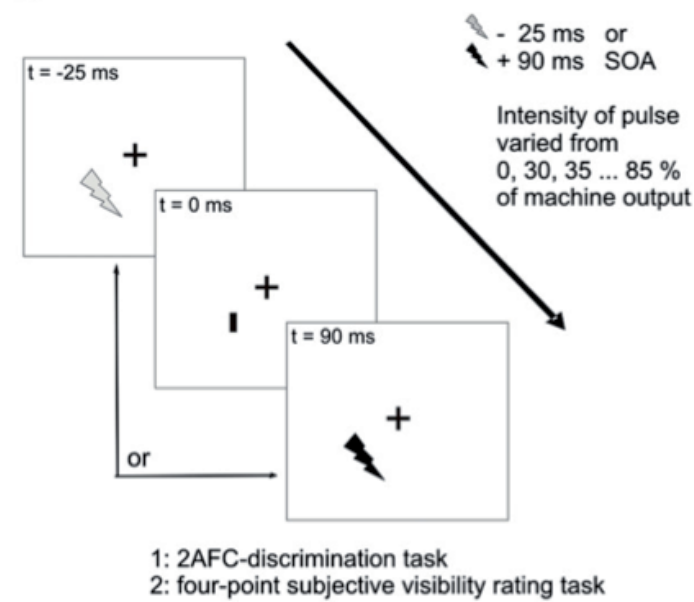

C Pre-stimulus masking window Post-stimulus masking window<smiles>COc1ccccc1</smiles>

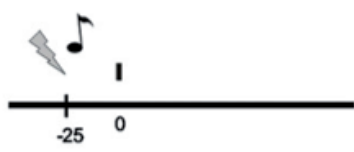

or
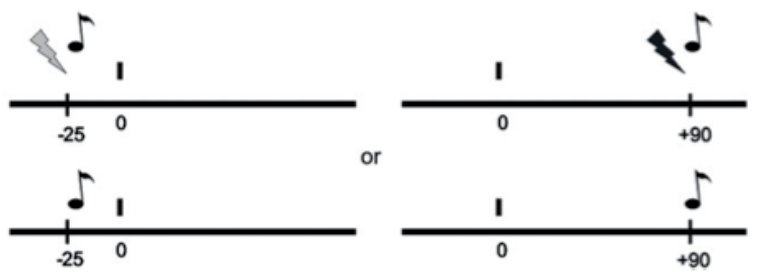

Figure 1: Stimulus and design

(A) Our stimuli consisted of bars, which could be of either horizontal or vertical orientation. They were very small and difficult to perceive even without TMS, see Section 2 for details. (B) Schematic depiction of the experimental design is shown. There were principally two factors; the pulse timing was either post-stimulus $(90 \mathrm{~ms})$ or pre-stimulus $(-25 \mathrm{~ms})$, where 'stimulus' refers to the onset of the visual target stimulus. Orthogonally, magnetic stimulation intensity was varied from very low $(30 \%)$ to high ( $85 \%$ ) levels, in steps of $5 \%$. On any given trial, participants were required to first indicate (or 'guess') the orientation of the stimulus in a 2-alternative forced-choice (2AFC) task. Subsequently, they were asked to indicate on a four-point scale the subjective visibility of the stimulus on that same trial. (C) A schematic depiction of all possible trials is shown. As indicated, pulses either preceded or came after the visual stimulus (' 0 ' on the horizontal timelines). The pulses were either real TMS, or SHAM TMS (the music symbol only). Not shown is the experimental factor of intensity. 
(Bullier, 2001; Hochstein \& Ahissar, 2002; see Chapter II.5).

One reason some experiments could dissociate between subjective and objective measures of vision, while others would not, might be the stimulation intensity, or TMS 'dosage', used. Perhaps subjective and objective measures of vision are differentially sensitive to TMS disruption, which could mean that only a narrow band of stimulation intensities would reveal a dissociation. It seems that no study to date has systematically and in detail investigated the effects of TMS stimulation intensity on visual processing, irrespective of the measure of vision, with high 'dosage-resolution'. Usually a single intensity is chosen and fixed throughout the experiment, while it seems wholly possible that different intensities lead to different TMS masking curves (see Beckers \& Homberg, 1991; Kammer, Puls, Erb, \& Grodd, 2005; Reichenbach, Whittingstall, \& Thielscher, 2011 for some investigations of TMS dosage effects). Perhaps differential selection of TMS intensities may also contribute to the inconsistency between reports on masking time windows other than the consistent 90 $100 \mathrm{~ms}$ window. Perhaps at certain intensities TMS masking at time window A will cooccur with masking in time window $\mathrm{B}$, while at other intensities only time window A yields masking effects, or only time window B.

In the current experiment we aimed to address these issues. We selected two time windows to investigate, namely the classical post-stimulus time window (+90 ms SOA) and the debated pre-stimulus time window ( $-25 \mathrm{~ms}$ SOA). We implemented an objective forced-choice visual task performance measure and a subjective stimulus visibility rating measure. We systematically varied the intensity of our single TMS pulses (from $30 \%$ to $85 \%$ of the machine output, in small increments of $5 \%$ - although we then smoothed the data over intensities). We moreover conducted additional SHAM TMS measurements, to control as much as possible for any non-neural effects of TMS. We thus evaluated the neural masking effects of TMS at post-stimulus and pre-stimulus time windows, on both subjective and objective measures of vision, across this range of TMS intensities.

\section{Materials and Methods}

\section{Participants}

Eight participants were tested in this study (four males, age range 22-28). No participants had abnormal vision or a history of neuropsychiatric disorders. They were screened by a medical supervisor prior to participation, and supplied written informed consent. The local medical-ethical committee approved the experiment.

\section{Stimuli and tasks}

Stimuli and tasks were exactly as in Chapter II.1. 


\section{Design and TMS parameters}

The experiment consisted of three 2-h sessions per participant. One session for the poststimulus time window (in which stimulus onset asynchrony (SOA) between the visual target stimulus and the TMS pulse was $90 \mathrm{~ms}$ ), one session for the pre- stimulus time window (in which SOA was -25: TMS pulse administered $25 \mathrm{~ms}$ before visual stimulus onset), and one session for both SHAM TMS measurements. In the TMS sessions (poststimulus and pre-stimulus), a total number of 15 trials per intensity were measured. There were 12 intensity conditions: $30 \%, 35 \%, 40 \%, 45 \%, 50 \%, 55 \%, 60 \%, 65 \%, 70 \%$, $75 \%, 80 \%, 85 \%$ of machine output (MC-B70 butterfly coil). ${ }^{7}$ See Fig. 1A for the stimulus, and Fig. 1B for a schematic illustration of the design. These intensities were presented randomly across trials. Since our experimental setup did not allow a randomization and trial-by-trial variation of stimulation intensities, we prefabricated lists of intensities to be used and shuffled those. One and the same trained experimenter then went through this list during the experiment and manually changed the intensity on the TMS machine for each new trial. Participants wore ear plugs to make sure they could not hear the preparatory TMS machine noises that might otherwise predict to an extent the strength of the upcoming pulse, and they indeed reported that they were unable to 'guess' which intensities (high or low) would come next. Interleaved randomly with these intensities were no-TMS trials, in which the machine output was set to 0 . While an experimenter changed the intensities, Presentation (Neurobehavioral systems, CA, USA) software presented both visual stimuli and controlled TMS pulses timing. A total number of 195 trials per session were measured.

To control for non-neural effects of TMS, such as the sensation of a coil on the head, the suspense of incoming pulses, and the sound of a TMS pulse, we administered SHAM TMS. We considered that the timing of the pulse sound might have a significant influence, so to most rigorously control for the non-neural TMS effects we measured SHAM TMS trials in both time windows, but in the same session. A list of intensities was again used, but adapted so that the loudness of the TMS pulse 'clicks' matched those of the actual TMS intensities. This yields a range of SHAM intensities to compare the TMS results to. In the SHAM TMS session we measured 11 trials per intensity, and did not measure intensity $30 \%$ because our SHAM setup could not reliably produce a sound equal in loudness to the TMS coil at $30 \%$. Moreover, time-constraints were considered since both post- and pre-stimulus time windows were measured in one session. This procedure should have provided us with sufficient control data, if SHAM TMS had no consistent effects on performance. The results bear this out, as can be seen under "Results".

\footnotetext{
${ }^{7}$ For the post-stimulus session, one participant was not tested at $85 \%$, and along with an additional subject not tested at $30 \%$ or $35 \%$. In these earliest two measurements, because the subjects reported seeing nothing at intensities well above $85 \%$, and seeing everything at intensities well below $40 \%$ respectively, we considered saving time and subject aggravation by focusing on the 'border' intensities of interest. However, we abandoned this idea in the interest of consistency, and proceeded to measure all intensities on all subjects in all sessions as reported, regardless of how strong or weak the masking effects at the extreme ends of the intensity scale.
} 
Participants could pace themselves throughout the experiment, since a button press was required to continue to the next trial. This also ensured that the experimenter had enough time to set up the next intensity; she indicated her readiness to the participant. The time between trials was thus ensured to be around 6-8 s. Biphasic TMS pulses were administered over the occipital cortex with a figure- 8 coil (MC-B70). The coil handle was oriented laterally to the right, with initial current direction going away from the handle. The site of stimulation was determined by a phosphene localization procedure as in previous experiments (Chapters II.1,2).

\section{Analysis}

We effectively had two control measures for our TMS results. On the one hand, we had randomly interleaved no-TMS trials, and on the other hand we obtained SHAM TMS measurements. We provide the no-TMS results as they are (see Fig. 2), but decided to compare our TMS results to the SHAM TMS data, for two reasons. (1) SHAM TMS trials provided a control condition that was intensity-specific, and time windowspecific. (2) the no-TMS data were different from TMS and SHAM TMS trials, probably because the absence of a TMS pulse (sound) was generally unexpected. Thus, the no-TMS trials were almost 'oddballs' that may have interfered with the visual processing in unpredictable, and possibly time window-specific ways (the absence occurs before or after the stimulus, see de Graaf \& Sack, 2011, chapter III.2, for additional considerations of controls in TMS research).

Our experiment, with two TMS time windows multiplied by 13 intensities (noTMS and 12 intensities) is characterized by a vast amount of experimental conditions. This is not uncommon in TMS masking experiments, and in such cases traditional correction for multiple comparisons is not always possible. However, we first did analyze our data with full-factorial repeated-measures ANOVAs (Huynh-Feldt corrected). These included the factors TMS (TMS and SHAM), SOA (masking window: pre-stimulus and post-stimulus), and INT (intensity: 35-85\%, since SHAM did not include $30 \%$ ). As described in "Results", to directly compare TMS effects on the subjective and objective measures of vision, in a post hoc analysis we normalized all data on the two measures of vision and performed another repeated-measures ANOVA that included the additional factor of MV (measure of vision; subjective and objective). After initial ANOVAs, we explored the TMS data and SHAM data over intensities using uncorrected paired-samples t-tests. We used two-tailed criteria for significance, even though one-tailed tests would have been justified, to be somewhat conservative in compensation.

To evaluate the patterns in the whole dataset, rather than focusing on planned individual comparisons only, we additionally performed a trend analysis on the data. Over intensities $0-85 \%$, for TMS and SHAM separately, and for pre-stimulus and poststimulus windows separately, repeated measures polynomial contrasts were calculated using SPSS statistical software. We only looked at linear and quadratic contrasts. Before all statistical testing, and represented in Fig. 2, we smoothed all the data over intensity levels. This was more representative, since the unsmoothed data were rather noisy both in SHAM and TMS conditions. This procedure consisted of a standard 
'sliding window' of averaging: each data point was averaged with its two neighbors on the scale of intensity (horizontal axes in Fig. 2). Note that this procedure decreased our effective 'intensity resolution' slightly, but this was not problematic for our interpretations and conclusions. Overall, this approach yielded very clear patterns of intensity effects, with strong TMS effects and small estimation errors (see Fig. 2).

\section{Results}

\section{Objective discrimination performance}

We first looked at the objective measure of vision. A repeated-measures ANOVA with factors TMS (TMS, SHAM), SOA (masking window: pre-stimulus and post-stimulus), and INT (intensities: from $35 \%$ to $85 \%$, smoothed - see Section 2), revealed a main effect of TMS ( $\mathrm{F}=29.04, \mathrm{P}=0.001$ ), a main effect of INT (intensity, $\mathrm{F}=8.98, \mathrm{P}<$ 0.001 ), but this effect predictably depended on whether TMS or SHAM TMS was applied, as evidenced by a significant interaction TMS x INT $(\mathrm{F}=13.95, \mathrm{P}<0.001)$. There was no main effect of SOA, and there were no significant interactions between SOA and other factors. However, there were some indications for differences between pre-stimulus masking and post-stimulus masking (see below), and a full-factorial ANOVA with so many levels and the current sample size can be suboptimal. Therefore we do present the data separately for post-stimulus and pre-stimulus masking windows to provide a full illustration of the data patterns.

Fig. 2A shows these results for the orientation discrimination performance. While SHAM TMS (red) has no effect on performance, ruling out non-neural unspecific effects of the stimulation protocol, TMS with increasing intensity has increasingly strong effects on performance. This is true for both the classical post-stimulus time window, and, interestingly, the pre-stimulus time window. The extent of masking (effect size) is very similar between the two time windows. Assuming that the poststimulus masking curve (over intensities) reflects neuronal masking effects, this overall similarity may support the notion that the pre-stimulus time window of masking is a 'real' masking effect. However, differences between the time windows can also be observed. The post-stimulus time window seems to show an unimpaired performance up to a certain point, a 'cut-off' around $65 \%$ TMS intensity, after which performance quickly decreases with intensity.

In contrast, the pre-stimulus curve suggests a more gradual decline in performance. Already at lower intensities, performance seems decreased as compared to SHAM TMS. Statistically, performance in the pre-stimulus masking window is significantly lower with TMS as compared to SHAM TMS from intensity 55\% (P< $0.05)$ and higher. In the post-stimulus time window, however, an intensity of $70 \%(\mathrm{P}<$ 0.05 ) or higher was needed for statistically significant masking. Intensities for which performance with TMS was significantly lower than performance with SHAM TMS are highlighted in yellow in Fig. 2, and all statistical results are specified in Table 1. 

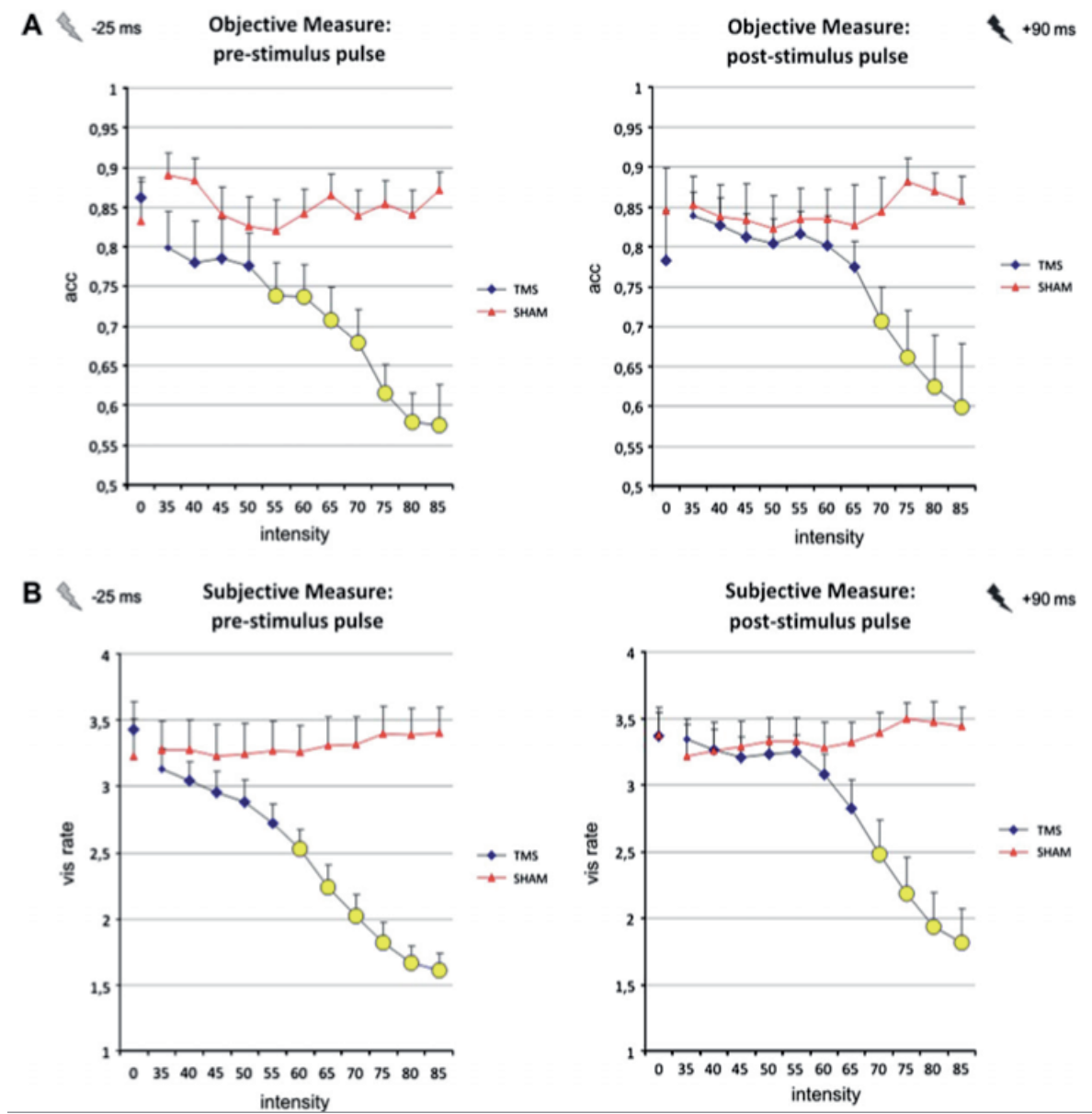

Figure 2: Results

(A) The smoothed masking curves over increasing intensity (horizontal axis) are presented for the two pulse timings (pre-stimulus left, post- stimulus right) separately, always for TMS (blue) and SHAM TMS (red) separately. On the vertical axis is performance on the orientation discrimination task, displayed as proportion correct. Error bars reflect standard error of the mean (across participants). Levels of intensity where TMS data differed significantly from SHAM TMS data are highlighted by yellow markers. Note that the level of intensity where TMS differs significantly from SHAM TMS is lower for pre- stimulus as compared to post-stimulus TMS. See main text for a discussion of trend analysis on these data. (B) Same as in A, except that the vertical axis reflects the subjective visibility rating on a four-point scale. Thus, the objective measure of vision is presented in A (see text), while here the subjective measure of vision is presented. Note the similarities in masking patterns over increased intensities between A and B.

Thus, methodologically we can conclude that the intensity selected does indeed affect the pattern of findings. If an intensity had been selected and fixed in the 'grey zone' between $50 \%$ and $70 \%$ intensity, which is in or at least near the range of 
intensities often used for TMS masking, ${ }^{8}$ masking effects might have been ambiguous, and results for both time windows might have yielded unrepresentative interpretations.

To go beyond individual comparisons, we performed trend analyses. Analysis of polynomial contrasts revealed a significant linear trend in the pre-stimulus TMS data (linear component: $\mathrm{F}=9.50 ; \mathrm{P}=0<0.05$; quadratic component: $\mathrm{F}=1.12 ; \mathrm{P}=0.318$ ), and a marginally significant quadratic trend in the post-stimulus TMS data (linear component: $\mathrm{F}=7.90 ; \mathrm{P}<0.05$; quadratic component: $\mathrm{F}=4.46 ; \mathrm{P}=0.073$ ). Although this latter statistical result did not reach the statistical threshold, it was significant for the subjective measure of vision, see below. Although these findings are not direct statistical comparisons of trends, these results form the statistical basis of the earlier observations of a gradual effect of TMS for pre-stimulus, but a sudden effect (after a certain cut-off) for post-stimulus time windows.

Table

Statistical results for all comparisons.

\begin{tabular}{|c|c|c|c|c|c|}
\hline \multicolumn{3}{|c|}{ Post-stimulus masking window } & \multicolumn{3}{|c|}{ Pre-stimulus masking window } \\
\hline Intensity & $t$-Value & $P$-value & Intensity & $t$-Value & $P$-value \\
\hline \multicolumn{6}{|c|}{ Objective measure (performance on forced-choice orientation discrimination task) } \\
\hline No-TMS & -1.5679 & 0.160892 & No-TMS & 0.559143 & 0.59349 \\
\hline 35 & -0.40339 & 0.6987 & 35 & -1.58602 & 0.156756 \\
\hline 40 & -0.31239 & 0.763846 & 40 & -1.7171 & 0.129663 \\
\hline 45 & -0.52524 & 0.615634 & 45 & -1.03023 & 0.337177 \\
\hline 50 & -0577 & 0.582013 & 50 & -1.14262 & 0.290767 \\
\hline 55 & -0.44337 & 0.670879 & 55 & -2.38801 & 0.04831 \\
\hline 60 & -0.62806 & 0.549899 & 60 & -2.60857 & 0.034986 \\
\hline 65 & -0.97004 & 0.364343 & 65 & -2.79497 & 0.026717 \\
\hline 70 & -2.99277 & 0.020147 & 70 & -2.46126 & 0.043385 \\
\hline 75 & -4.74165 & 0.002104 & 75 & -4.49167 & 0.002826 \\
\hline 80 & -3853 & 0.006268 & 80 & -6.29737 & 0.000405 \\
\hline 85 & -3.09315 & 0.01749 & 85 & -5.86886 & 0.000619 \\
\hline \multicolumn{6}{|c|}{ Subjective measure (stimulus visibility ratings on 4-point scale) } \\
\hline No-TMS & -0.11467 & 0.911928 & No-TMS & 1.772429 & 0.119611 \\
\hline 35 & 1.075919 & 0.317642 & 35 & -0.94394 & 0.376633 \\
\hline 40 & 0.091967 & 0.929301 & 40 & -1.41556 & 0.199821 \\
\hline 45 & -1.13455 & 0.293915 & 45 & -1.28217 & 0.240611 \\
\hline 50 & -0.86243 & 0.417017 & 50 & -1.45947 & 0.187807 \\
\hline 55 & -0.59547 & 0.570273 & 55 & -2.03665 & 0.081113 \\
\hline 60 & -0.89311 & 0.401459 & 60 & -2.73064 & 0.029312 \\
\hline 65 & -2.05242 & 0.079247 & 65 & -3.41089 & 0.011274 \\
\hline 70 & -3.10114 & 0.017295 & 70 & -1.6543 & 0.004213 \\
\hline 75 & -4.51922 & 0.002735 & 75 & -4.79629 & 0.001975 \\
\hline 80 & -5.4928 & 0.000913 & 80 & -5.82862 & 0.000644 \\
\hline 85 & -5.9551 & 0.000567 & 85 & -6.07758 & 0.000502 \\
\hline
\end{tabular}

Displayed are the results of uncorrected paired-samples t-tests between TMS and SHAM TMS. Bold values indicate comparisons significant in two-tailed analysis, values in italics indicate comparisons significant in one-tailed analysis.

\section{Subjective visibility ratings}

For subjective visibility ratings, a repeated-measures ANOVA with factors TMS (TMS, SHAM), SOA (masking window: pre-stimulus and post-stimulus), and INT (intensities:

\footnotetext{
${ }^{8}$ Note that at least the Medtronic and the Magstim systems differ in their generated intensities (Thielscher \& Kammer, 2004). Thus, 50-70\% machine output on our system may correspond to higher values on the Magstim system.
} 
from $35 \%$ to $85 \%$, smoothed - see Methods), revealed a main effect of TMS ( $F=13.66$, $\mathrm{P}<0.01$ ), a main effect of INT (intensity, $\mathrm{F}=13.59, \mathrm{P}<0.01$ ), though this effect again depended on whether TMS or SHAM TMS was applied, as evidenced by a significant interaction TMS x INT $(\mathrm{F}=38.82, \mathrm{P}<0.001)$. There was now also a main effect of SOA $(\mathrm{F}=6.21, \mathrm{P}<0.05)$, and there was a trend for an interaction between SOA and TMS $(\mathrm{F}=4.03, \mathrm{P}=0.085)$. There was no three-way interaction between TMS, SOA, and INT. Thus, a difference in visibility ratings existed between the pre-stimulus masking window and the post-stimulus masking window, and the interaction with TMS was marginally significant even in the strict full-factorial ANOVA. Therefore we again present the data separately for post-stimulus and pre-stimulus masking windows to provide a full illustration of the data patterns.

Fig. 2B shows the subjective ratings participants made over intensities, in the same trials as the performance on the orientation discrimination task (shown in Fig. 2A). While reports have been published about diverging TMS masking effects on subjective and objective measures for some tasks and time windows (e.g. Boyer et al., 2005; Koivisto et al., 2010), the pattern of TMS effects over intensities on subjective visibility ratings closely corresponds to the objective performance in our dataset. This sort of comparison is of course of a very different kind than the analyses published previously, but considering the very different nature of our subjective task and objective performance task, it is striking how closely the graphs resemble each other. For the post-stimulus time window, visibility ratings seemed unaffected up to intensities around $60 \%$ of the machine output. Then, with increasing intensity the subjective stimulus visibility seems to drop rather quickly.

As similar as the objective and subjective measures of post-stimulus TMS masking are to each other, so different are the TMS effects between the post-stimulus and the pre-stimulus masking windows. For the objective task performance, we already noted that TMS masking effects seemed to require lower TMS intensities for the pre-stimulus window, in our paradigm. This difference is not only mirrored in the subjective visibility ratings, but even more pronounced. While the post-stimulus window seems to reveal a sharp cut-off of visibility at a certain threshold intensity $(70 \%$ for these stimuli), the pre-stimulus window reveals a very gradual, more linear effect of TMS intensity on stimulus visibility. It seems pre-stimulus masking occurred already at lower intensities, as compared to the post-stimulus time window. As in the performance data, this observation is confirmed statistically. While for the post-stimulus time window, TMS masking of subjective visibility is significant for intensity $70 \%(\mathrm{P}<$ 0.05 ) and higher, for the pre-stimulus time window, TMS masking of subjective visibility requires only $60 \%(\mathrm{P}<0.05)$ and higher. We again refer to Table 1 for all statistical values.

A significant linear trend was present in the pre-stimulus TMS data, over intensities (linear component: $\mathrm{F}=42.14 ; \mathrm{P}<0.001$; quadratic component: $\mathrm{F}=1.16 ; \mathrm{P}=$ 0.318). A significant quadratic trend, alongside a significant linear component, was present in the post-stimulus TMS data (linear component: $\mathrm{F}=19.57$; $\mathrm{P}<0.01$; quadratic component: $\mathrm{F}=19.11 ; \mathrm{P}<0.01)$. These statistical results seem to support the observation that different patterns are apparent in the masking effects with increasing 
intensity, for pre-stimulus versus post-stimulus TMS pulses. But note, as above, that this analysis is not a direct statistical comparison of trends in both time windows.

\section{Objective versus subjective measures of vision}

In a post hoc analysis we standardized the behavior on both tasks for all participants, resulting in scores in a common space. A full-factorial ANOVA now including the factor MV (measure of vision) revealed that, as seemed evident from the data in Fig. 2, there was no difference between both measures of vision. There was no main effect of MV $(F=0.072, P=0.796)$ and there were no significant interactions with others factors.

We performed one additional analysis in which we looked at the pattern of objective task performance for non-perceived trials only (in which subjective rating was 1 or 2), over intensities, for TMS and SHAM. Arguably, this type of analysis comes closest to an investigation of 'conscious' versus 'unconscious' vision and TMS effects thereon. We present the results of this analysis in Supplementary material because of methodological constraints, but can mention here that while performance was often above-chance on these non-perceived trials in the TMS condition (conform 'TMSinduced blindsight'), the performance was generally even higher above-chance on these non-perceived trials in the SHAM condition (not conform 'TMS-induced blindsight').

\section{Pre-stimulus masking versus post-stimulus masking}

The trend analyses for the pre-stimulus masking window and the post-stimulus masking window, presented above for both measures of vision separately, provide a first indication for differences between both masking windows and therefore different neuronal mechanisms. However, so far we did not discuss direct comparisons between both masking windows.

For performance, direct analyses between masking windows yielded no significant results. For visibility ratings, the subjective measure of vision, there was already a significant effect of factor SOA (masking window) in the full-factorial ANOVA. In a follow-up analysis that focused on only the TMS conditions (not including SHAM TMS data), the strict ANOVA (SOA x INT) yielded not only a significant effect of INT $(\mathrm{F}=24.14, \mathrm{P}<0.001)$ but also a marginally significant main effect of SOA $(\mathrm{F}=5.06, \mathrm{P}=0.059)$ and interaction between SOA and Intensity $(\mathrm{F}=$ $3.22, \mathrm{P}=0.061)$. Follow-up paired samples t-tests comparing the pre-stimulus masking window with the post-stimulus masking window on each level of TMS intensity revealed significant differences between both masking windows on the intensities $50 \%$ $(\mathrm{t}=2.62, \mathrm{P}<0.05), 55 \%(\mathrm{t}=3.80, \mathrm{P}<0.01), 60 \%(\mathrm{t}=3.52, \mathrm{P}<0.01), 65 \%(\mathrm{t}=2.85, \mathrm{P}$ $<0.05)$, with marginally significant differences on intensities $45 \%(\mathrm{t}=2.07, \mathrm{P}=0.08)$ and $70 \%(\mathrm{t}=1.95, \mathrm{P}=0.09)$. These statistical results of course reflect the earlier observation that TMS effects on vision seemed to take effect at lower intensities in our paradigm, and more gradually, in the pre-stimulus masking window as compared to the post-stimulus masking window. Please note that we advocate a careful interpretation 
here, and only take these findings to provide a first indication of different mechanisms underlying pre-stimulus TMS masking and post-stimulus TMS masking.

In summary, (1) TMS-induced masking occurred in both pre-stimulus and post-stimulus masking windows. (2) Masking effects were very similar for both the objective and subjective measure of vision, and we found no statistical differences between them. (3) There were some first indications that masking effects over intensities differed between pre-stimulus and post-stimulus masking.

\section{Discussion}

We here found no dissociation between TMS effects on subjective or objective measures of visual processing. This was not a matter of ambiguity or lack of power. Rather, the similarities between the TMS effects over intensities, on a forced-choice visual task performance and a fundamentally different measure involving subjective ratings of stimulus visibility, were striking. All the more so because we had two windows of TMS masking to evaluate a pattern of effects for, and these differed very similarly for both our measures of visual processing. Previous reports suggested that TMS can disrupt conscious, but not 'unconscious' forced-choice performance of orientation stimuli (Boyer et al., 2005). These authors explained their results by proposing that the forced-choice performance was mediated by extrageniculate pathways bypassing $\mathrm{V} 1$, but that intact V1 processing was necessary for visual awareness. If so, our experiment should have yielded a pattern of decreasing visibility ratings with increasing intensity, but unhampered performance - presumably in both time windows. Others suggested that in the 'classical' masking time window, a feedback stream mediating conscious vision might be disrupted by TMS (Lamme, 2006), while a feedforward stream mediating task performance had already 'sweeped through' unhampered. In such a scenario, we should have expected decreasing visibility ratings with increasing intensity but unhampered objective task performance for the post-stimulus time window, while we should have obtained similar effects on both measures of vision for the pre-stimulus time window. Our results are not in line with either of these scenarios.

Our pattern of findings, with equal effects of TMS on subjective and objective measures of vision, would however be predicted by several models of vision. On the one hand our findings would be in line with any feedforward-feedback model that proposes that the feedforward stream passes early visual cortex around $100 \mathrm{~ms}$. However, this would mean that the classical time window marks the first functionally relevant arrival of visual information in these regions, which seems late (Kammer, 2007b; Lamme, 2006; Lamme \& Roelfsema, 2000) and seems to go against several studies suggesting earlier TMS masking windows (Corthout et al., 1999; Kammer, 2007b; Laycock et al., 2007; Paulus et al., 1999). It remains possible that the mask- ing effect at $100 \mathrm{~ms}$ does represent disruption of a recurrent, feedback, information stream, but that the stimuli we used might not afford enough information to the feedforward sweep to allow correct task performance, and thus dissociation between conscious and unconscious vision. Note, however, that our stimuli were similar to those used by Boyer et al. (2005). 
In contrast, as discussed in the Introduction, our findings are in line with recurrent models of vision that do not ascribe a visual awareness role to the specific feedback stream that arrives around $100 \mathrm{~ms}$. In various recurrent models, the feedback stream has other functions (Bullier, 2001; Hochstein \& Ahissar, 2002), such as filling in the details of the visual image in the Reverse Hierarchy model (Hochstein \& Ahissar, 2002). Another possibility, would be the existence of multiple feedback streams, as indeed multiple stages of early visual cortex involvement have been shown in visual processing (e.g. Roelfsema, Tolboom, \& Khayat, 2007). It could moreover be that our $+90 \mathrm{~ms}$ SOA was 'too early' to disrupt the later part of the feedback phase - if indeed the first part of the classical masking window corresponds to feedforward activity while the later part reflects recurrent processing (see previous chapter and II.5).

\section{TMS intensity and visual suppression}

Our data show that visual suppression by TMS is possible in time windows other than the 'classical' window around 90-100 ms. This has been proposed for various alternative time windows (see Chapter II.5). We find this pre-stimulus time window particularly interesting, because a TMS masking effect here would likely involve a fundamentally different neuronal mechanism compared to post-stimulus time windows. After all, TMS pulses after the visual stimulus likely disrupt the ongoing processing of that stimulus, for instance by adding neural noise. In contrast, a TMS pulse before the visual stimulus may put the 'system' of the brain in a certain state: a state of the visual system not conducive to the processing of a following stimulus. Because of this, we feel that the pre-stimulus masking window could prove very informative if investigated further. Thus far, it does not yet seem to be commonly accepted as a true masking window in which TMS pulses instigate neuronal processes of visual suppression. Yet our data support the notion of pre-stimulus masking. We do not believe that nonneuronal processes could yield the gradual pattern of effects shown here, and we controlled for many potential non-neuronal confounders by using SHAM-TMS in the same time window. Yet further research, aiming to replicate our pre-stimulus masking result while controlling for retinotopic specificity and/or TMS stimulation site, would be valuable.

\section{Supplementary Material}

\section{Behavioral performance on trials that were not consciously perceived}

As discussed in the main manuscript, it seemed possible that conflicting reports about TMS-induced blindsight might be due to different levels of TMS intensity. Some intensities might result in dissociations while other do not.

In a post hoc analysis we separated the trials that were consciously perceived (subjective rating of 3 or 4 - per the instructions), from the trials that were not 
consciously perceived (subjective rating of 1 or 2 - per the instructions). We then plotted across intensities the behavioural performance for those trials that were not consciously perceived. We did this for both SHAM TMS (where the difficult stimuli were sometimes, though not often, 'missed') and real TMS. Presumably, scenarios of TMS-induced blindsight would predict that behavioural performance is above-chance, potentially for some intensities but not other, in the real TMS but certainly not in the SHAM TMS. After all, whichever source of (neural or environmental) noise caused stimuli to be non-perceived in the SHAM TMS session, should affect the visual system holistically. In the case of real TMS, in contrast, the hypothesis is that the conscious processing specifically is disturbed, due to TMS pulses. In summary, we should expect above-chance performance for real TMS, and not - or at the very least less so - for SHAM TMS. As can be seen in supplementary figure 1, this was not what we found. We note that the number of trials in each condition was not very high - so we should refrain from drawing strong conclusions. However, the pattern is suggestive. At the very least, the high above-chance performance in SHAM TMS, across all intensities (even higher than real TMS performance) casts a shadow of doubt on the validity of using a subjective visibility report to label trials as lacking in conscious vision to subsequently draw strong conclusions about unconscious processing in a forced-choice task - and then attributing such a result to the TMS manipulation. We note that (although the number of trials is again smaller), this pattern was not noticeably different for subjective ratings of 1 versus 2 .

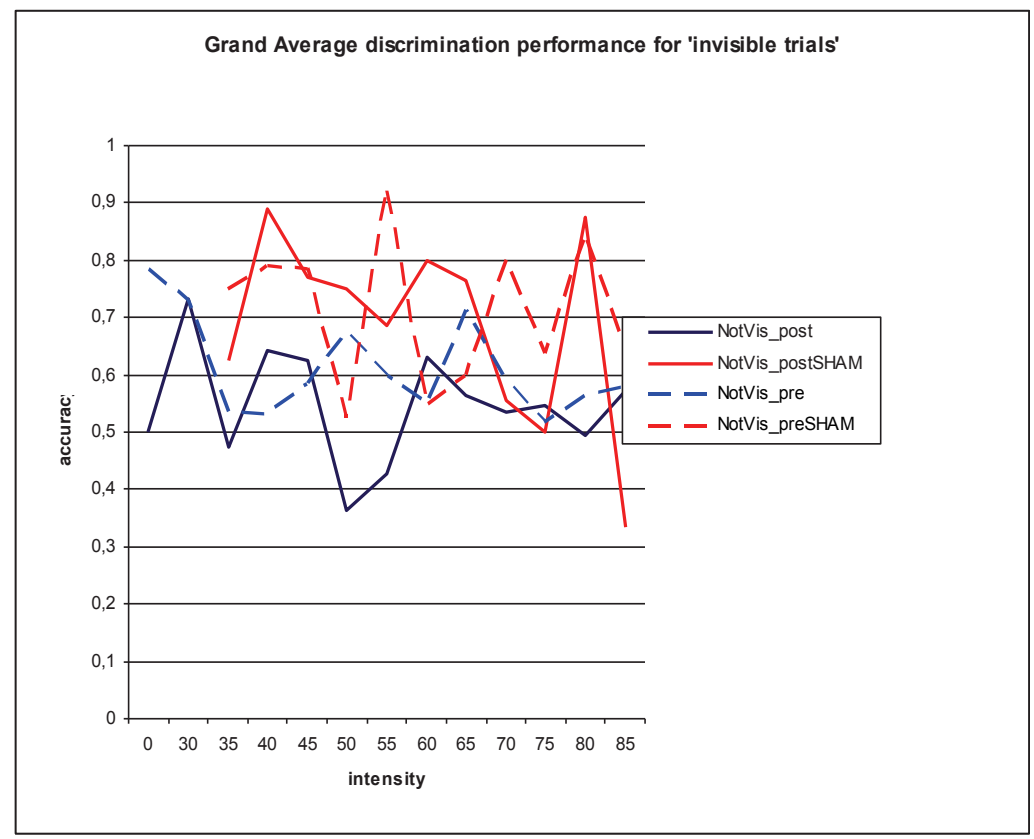

Figure S1: performance in non-perceived trials

Red lines indicate SHAM TMS condition, blue lines indicate real TMS condition. Dashed lines reflect the pre-stimulus masking window, straight lines the post-stimulus masking window. 



\section{II.4}

\section{On the Masking Effects of Occipital}

TMS with Varying Intensity when

Applied Before and After Visual Stimuli

(II)

Related publication(s):

De Graaf, TA*, Jacobs, C *, Sack, AT (in preparation). TMS masking of objective and subjective vision is always equal, but not always retinotopically specific

* equal contribution 


\begin{abstract}
In previous work, we explored the effects of occipital TMS on both objective and subjective measures of visual processing across a range of stimulation intensities. TMS at higher intensities successfully masked visual images subjectively and disrupted visual task performance, both when applied in the 'classical masking window' at +90 ms after the onset of a visual stimulus, and when applied prior to the onset of a stimulus. Such 'pre-stimulus masking windows' are counterintuitive, since no active neuronal processing of the sensory information yet takes place. To nevertheless establish the validity of pre-stimulus masking windows, we here evaluated the efficacy of single TMS pulses across a range of stimulation intensities at four time windows: at two pre-stimulus stimulation onset asynchronies (SOAs), $-50 \mathrm{~ms}$ and $-20 \mathrm{~ms}$, as well as two post-stimulus SOAs, $+90 \mathrm{~ms}$ and $+120 \mathrm{~ms}$. Crucially, we presented visual targets in the visual field location corresponding to the TMStargeted occipital region, but also in a control location. Any non-neural or non-specific effects would affect visual processing in both locations, while 'genuine' TMS masking effects should specifically disrupt visual processing of the targeted location. We found that TMS with increasing intensity linearly disrupted visual processing in the targeted visual field location for the classical $+90 \mathrm{~ms} \mathrm{SOA}$, as well as for the $-20 \mathrm{~ms} \mathrm{SOA}-$ establishing this time window as a true masking window. Non-specific effects were observed for SOA $-50 \mathrm{~ms}$, and weaker effects (i.e. masking at only very strong intensity) were obtained for $+120 \mathrm{~ms}$. For all SOA's, effects on subjective and objective measures of vision were highly similar. These findings have methodological as well as theoretical implications for TMS masking and for models of vision and visual awareness.
\end{abstract}




\section{Introduction}

Transcranial magnetic stimulation (TMS) is a brain interference tool that allows the online disruption of functionally relevant neuronal activity. If applied over occipital cortex, this can lead to a complete abolishment of conscious perception of visual stimuli. This effect is well-established for TMS applied over a specific time window, namely at stimulus onset asynchronies (SOAs) around +80-100 ms (Amassian, Cracco, \& Maccabee, 1989; Beckers \& Homberg, 1991; Kammer, 2007). We have referred to this as the 'classical masking window' (de Graaf, Cornelsen, Jacobs, \& Sack, 2011; de Graaf, Goebel, \& Sack, 2012; de Graaf, Herring, \& Sack, 2011), and the visual suppression effect in this paradigm has been referred to as 'TMS masking' (Kammer, 2007, 2008).

However, there have been reports of TMS masking in time windows other than the classical $+90 \mathrm{~ms}$ window. TMS pulses around 30-40 ms (Corthout, Hallett, \& Cowey, 2002, 2003; Corthout, Uttl, Walsh, Hallett, \& Cowey, 1999; Corthout, Uttl, Ziemann, Cowey, \& Hallett, 1999; Paulus, Korinth, Wischer, \& Tergau, 1999), 180-220 ms (Camprodon, Zohary, Brodbeck, \& Pascual-Leone, 2010), and even beyond $300 \mathrm{~ms}$ (Chambers, Allen, Maizey, \& Williams, 2012) can disrupt visual task processing depending on tasks and stimulus parameters. What these SOAs have in common, is that they are 'post-stimulus': the visual information has entered the brain and its processing is likely disrupted by the TMS pulses. In the current study, we focus not only on poststimulus masking effects, but also 'pre-stimulus' masking windows, as demonstrated in the previous chapter. Corthout and colleagues suggested that TMS pulses prior to a visual stimulus can disrupt its processing (Corthout, Hallett, \& Cowey, 2003; Corthout, Uttl, Juan, Hallett, \& Cowey, 2000; Corthout, Uttl, Walsh, Hallett, \& Cowey, 1999; Corthout, Uttl, Ziemann, Cowey, \& Hallett, 1999), and we have recently replicated this phenomenon (de Graaf, Cornelsen, Jacobs, \& Sack, 2011; Jacobs, De Graaf, Goebel, \& Sack, 2012; Jacobs, Goebel, \& Sack, 2012).

The problem with pre-stimulus masking windows is two-fold: 1) it is not straightforward how the TMS pulses should mechanistically disrupt visual processing of a stimulus that is not yet even presented, 2) TMS pulses prior to visual stimuli may elicit all kinds of non-neural or non-specific effects, such as attentional priming, multisensory priming/integration (due to the 'click' of the pulse), and most importantly the induction of eye blinks. In previous work, we already addressed several of these alternative explanations of pre-stimulus masking effects, for instance by removing trials with eye-blinks (Jacobs, Goebel, \& Sack, 2012), controlling for sound with SHAM TMS (de Graaf, Cornelsen, Jacobs, \& Sack, 2011; Jacobs, De Graaf, Goebel, \& Sack, 2012), and controlling for sensory stimulation of the skin with vertex TMS (Jacobs, De Graaf, Goebel, \& Sack, 2012). Others have used high-resolution eye-tracking to elucidate the possible induction of eye movements (Corthout, Hallett, \& Cowey, 2011).

The issue remains, however, unsettled for two reasons. First, there are possibly two separate pre-stimulus time windows in which TMS disrupts visual processing, one around $-50 \mathrm{~ms}$, and one around $-20 \mathrm{~ms}$ (see Corthout references above). Second, while control site-TMS, SHAM TMS, and eye blink removal all control for various relevant potential confounders, an additional control could be enlightening in light of the results 
obtained thusfar (see Discussion). TMS masking involves presentation of visual stimuli to a location in the visual field that corresponds to the brain area targeted by the pulses. Including a control location in the visual field should reveal whether previously obtained effects were, though not necessarily non-neural, at the very least non-specific. The current study implemented this condition, which essentially involves repeating all experimental conditions for a second stimulus location.

In this work, then, we repeated the experimental procedures described in the previous chapter (de Graaf, Cornelsen, Jacobs, \& Sack, 2011), stimulating occipital cortex with single TMS pulses over a range of stimulation intensities. However, we presented visual stimuli at the targeted location and a control location. Moreover, we applied TMS at both of the potential pre-stimulus windows $(-50 \mathrm{~ms}$ and $-20 \mathrm{~ms})$, as well as the classical post-stimulus window $(+90 \mathrm{~ms})$ and a second, 'late' post-stimulus window $(+120 \mathrm{~ms})$. This should allow us to establish definitively whether TMS masking effects reported for both pre-stimulus SOAs are genuine or not.

\section{Materials and Methods}

\section{Participants}

Eleven participants volunteered for this study. Two were authors in this paper (T.G., C.J.) and one was aware of the goals and purposes of this study. The rest were fully naïve. No participants had abnormal vision or a history of neuropsychiatric disorders. They were screened by a medical supervisor prior to participation, and supplied written informed consent. The experimental protocols were approved by the local medicalethical committee.

\section{Stimuli, Tasks, and Procedure}

Stimuli involved rectangular bars measuring $\sim 0.035$ by $\sim 0.025$ visual degrees, presented at two possible locations. One location, as in previous experiments, was to the lower left of the fixation point (participants were seated in a chin rest and fixated throughout experimental trials), at an eccentricity of four visual degrees. The control location was diagonally symmetrical: to the upper right of the fixation point, at the same eccentricity. Bars were either horizontal or vertical, and were greyscale. Their luminance was not fixed. Participants started the experimental session with a calibration phase, in which they performed a visual discrimination task on the horizontal vs vertical (2-alternative forced-choice: 2AFC) bars (using keyboard button presses: left index finger on ' $Z$ ' key for vertical bars, right index finger on ' $/$ ' key for horizontal bars). They performed 20 trials for 9 levels of brightness of the bars, so that the performance over stimulus brightness could be plotted, separately for the lower left (experimental) stimulus location and upper right (control) stimulus location. Separately for the upper right and lower left location, the bars with brightness leading to performance closest to each other and to $90 \%$ correct discrimination were selected. We thus at the same time ensured equal difficulty of the tasks for both stimulus locations, and prevented ceiling 
effects on performance. All stimuli, in all phases of the experiment, were presented for 2 frames at a refresh rate of $60 \mathrm{~Hz}$ on a standard TFT monitor (Samsung SyncMaster): $33.4 \mathrm{~ms}$, using Presentation software (Neurobehavioral Systems, CA, USA).

In the actual experiment, participants performed the same 2AFC discrimination task, and a subjective stimulus orientation visibility judgment task rating the visibility of the orientation on a scale of 1 to 4 . We emphasized that it was not a confidence rating, but explicitly a visibility rating. We gave the same guidelines as in previous experiments (de Graaf, Cornelsen, Jacobs, \& Sack, 2011; de Graaf, Goebel, \& Sack, 2012; de Graaf, Herring, \& Sack, 2011), but emphasized that the guidelines were not as important as consistency in subjective criteria throughout the experiment. Since the experimental session was rather long, trials were not self-paced but fixed to jittered durations of 5-7 seconds. There were three breaks in the session, in which participants were invited to move and rest their eyes.

\section{TMS parameters and Design}

TMS was administered using a figure-8 coil (MC-B70) on a MagPro X100 stimulator. Single biphasic TMS pulses were administered to occipital cortex with the handle pointing outwards and the initial current going in lateral-medial direction. All conditions were measured in a single experimental session, consisting first of a calibration phase (see above), then a localization phase, then the experimental measurement. Localization was done using the phosphene localization methods described in previous experiments (de Graaf, Cornelsen, Jacobs, \& Sack, 2011; de Graaf, Goebel, \& Sack, 2012; de Graaf, Herring, \& Sack, 2011). TMS thus targeted the lower-left region of the visual field where visual targets appeared on half of the trials. To prevent order-effects, yet prevent also undesirable surprise-effects, trials with visual targets in the targeted lower-left region (LL) were presented in short blocks, as were targets appearing in the non-targeted upper-right (UR) region. These blocks were pseudo-randomized throughout the experiment, and were always announced with a short instruction screen, announcing the current target location. Subjects were then instructed to maintain fixation but covertly attend the thus cued location throughout the block.

Aside from the stimulus position condition (StimPos), we implemented a SOA condition with four levels: TMS pulses administered at $-50,-20,+90 \mathrm{~ms},+120 \mathrm{~ms}$, and a stimulation intensity condition (Int) with five levels $(35 \%, 50 \%, 60 \%, 70 \%, 80 \%$ of machine output). Stimulus-TMS SOAs were tested and confirmed in a separate calibration measurement session using a photodiode and oscilloscope recording the pulses to the TMS device and the actual appearance of stimuli in our experiment on screen, thus allowing us to correct for the difference in response delay at the two locations on the monitor. In contrast to during our previous experiment (de Graaf, Cornelsen, Jacobs, \& Sack, 2011), our setup now also allowed us to use serial communication between the stimulus PC and the TMS device to automatically set the TMS intensity for every trial, using Presentation software (Neurobiohavioral Systems, CA, USA). The intensity was thus pseudo-randomized throughout the experiment. In 
total, for all 40 condition cells (StimPos x SOA x Int), 16 trials were measured per participant, leading to a total of 640 trials per participant per session.

\section{Analysis}

We performed a full-model repeated-measures ANOVA (RM-ANOVA) with factors StimPos, SOA, and Int, separately for the accuracy (hit rate: proportion correct) over participants, and subjective visibility ratings. Follow-up tests were RM-ANOVAs and polynomial contrast analyses as reported in Results (all statistics Huynh-Feldt). To visualize and compare effects for both objective and subjective measures of vision, we standardized the results in all conditions for individual subjects to their individual mean and standard deviation (of results over all conditions) (i.e. Z-scores).

\section{Results}

\section{Objective discrimination performance}

The full-model RM-ANOVA with factors StimPos, SOA, and Int, revealed a three-way interaction $(\mathrm{F}=2.7 ; \mathrm{P}<0.01)$, indicating that the effects of stimulus position and intensity were dependent on SOA. We thus performed follow-up RM-ANOVAs separately for each time window. Shown in Figure 1 is average hit rate (proportion correct) over participants, plotted over levels of stimulation intensity, separately for the lower left (LL) and upper-right (UR) visual stimulus positions.

Figure 1C suggests that for the 'classical' masking window around $+90 \mathrm{~ms}$ there was a clear masking effect of TMS pulses, with increasingly disrupted visual task performance with increasing TMS intensity. This effect seems specific to stimuli on the lower left (LL). For UR, increasing TMS intensity appears to have no effect. Indeed, the RM-ANOVA showed a significant interaction StimPos $x$ Int $(F=3.1 ; \mathrm{P}<0.05)$. Followup RM-ANOVA confirmed that Int has an effect for LL stimuli $(\mathrm{F}=7.1 ; \mathrm{P}<0.001)$, but not for UR stimuli $(\mathrm{P}=0.496)$. Moreover, trend analysis revealed a significant linear component for $\mathrm{LL}(\mathrm{F}=21.7 ; \mathrm{P}=0.001)$, suggesting that increasing intensity linearly disrupts performance.

Figure 1D suggests that while there seems to be an effect of TMS at the highest intensities, specifically on LL, this is not as clear or pronounced as for the classical window. Statistically, there was no significant interaction between StimPos and Int, but follow-up analysis did yield a significant effect of Int on LL trials $(F=4.0$; $\mathrm{P}=0.01)$, though not at all on UR trials $(\mathrm{P}=0.475)$.

The two pre-stimulus time windows paint a very dissimilar picture. For the -20 ms SOA, there appears to be a clear masking effect that is specific for LL (Figure 1B). Indeed, RM-ANOVA revealed a significant interaction between StimPos and Int $(\mathrm{F}=5.0 ; \mathrm{P}<0.01)$. Follow-up tests showed a strong significant effect of Int for LL trials $(\mathrm{F}=7.5 ; \mathrm{P}=0.001)$, that moreover was significantly linear $(\mathrm{F}=13.1 ; \mathrm{P}<0.01)$. There was no effect of Int for UR $(\mathrm{P}=0.759)$. This suggests, in accordance with our previous 
results (previous Chapter), that TMS masking at this pre-stimulus time window constitutes 'true' masking, that is robust, strong, and retinotopically specific.

Interestingly, the latter is not the case for the $-50 \mathrm{~ms}$ window. Figure $1 \mathrm{~A}$ does show strong disruptions of visual task performance, as in our previous work (see above), but it does not seem to be specific to the targeted visual field location. Statistically, there was actually a significant interaction between StimPos and Int $(\mathrm{F}=3.4 ; \mathrm{P}<0.05)$, but follow-up analysis showed that while Int indeed had an effect on LL $(\mathrm{F}=8.1 ; \mathrm{P}<0.001)$, it also had a strong effect on $\mathrm{UR}(\mathrm{F}=11.2 ; \mathrm{P}<0.001)$. This suggests that the effects of TMS in this time window, with these stimuli, is not specific.

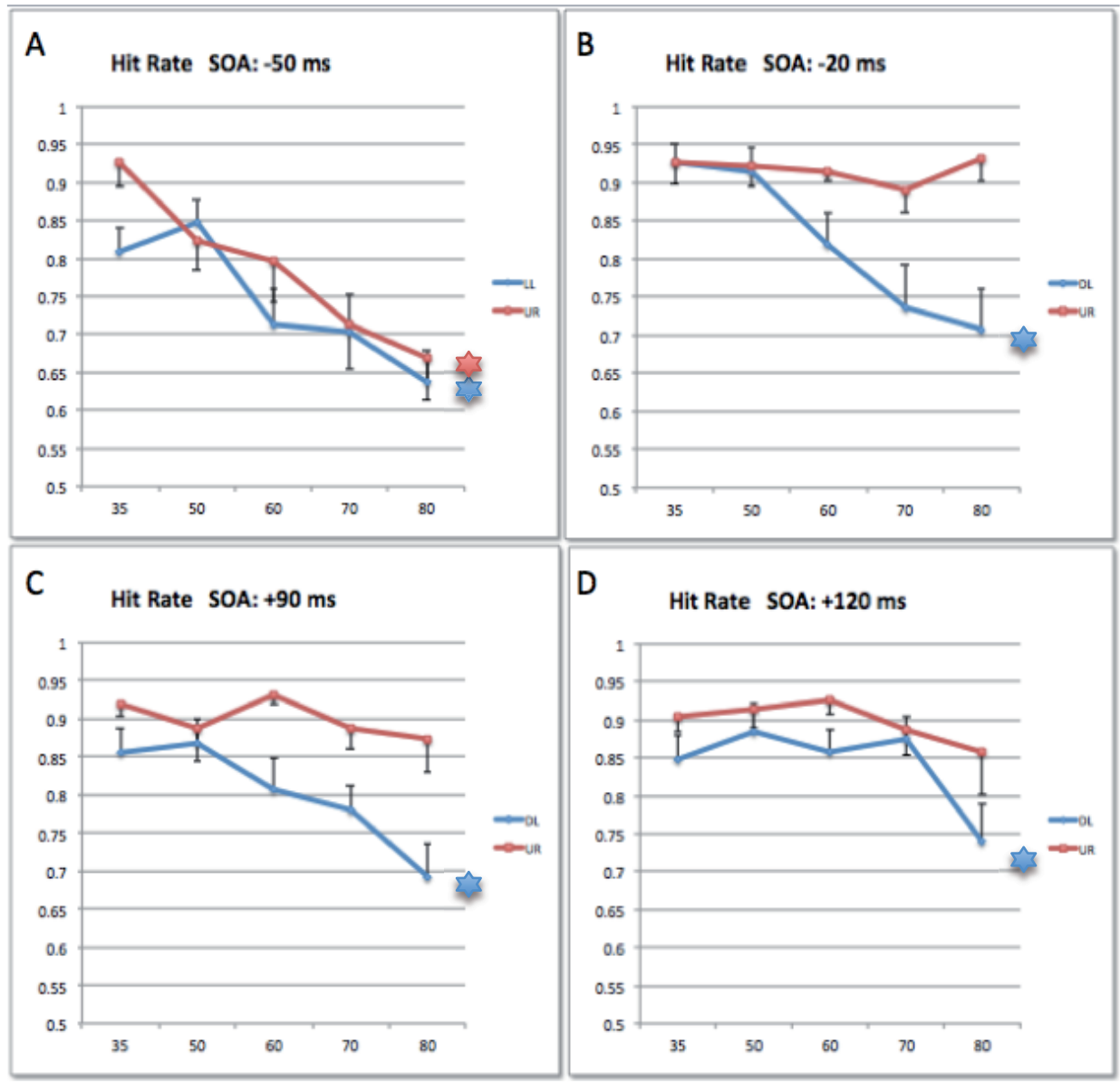

Figure 1: Results for Hit Rate

A. Plotted over TMS stimulation intensity (percentage of machine output on horizontal axis), separately for LL (blue lines) and UR (red lines), are hit rate for the SOA -50 ms. B. Same as in A but for SOA -20 ms. C. Same as in A but for SOA $+90 \mathrm{~ms}$. D. Same as in A but for SOA $+120 \mathrm{~ms}$. All error bars are standard error of the mean (SEM). Stars indicate that there was a significant effect of TMS intensity on hit rate (blue for targets LL, red for targets UR). 


\section{Subjective visibility ratings}

For subjective visibility ratings (participants indicated the subjective visibility of the stimulus' orientation on a scale of 1 to 4 , on every trial, after the objective 2AFC response), the full-model RM-ANOVA revealed a very strong 3-way interaction between StimPos $x$ SOA $x$ Int $(F=4.6 ; \mathrm{P}<0.001)$. We thus proceeded, similarly to the analysis for the objective discrimination performance, to analyze separately per masking time window. The patterns for these time windows are shown in Figure 2.

Figure $2 \mathrm{C}$ shows the visibility ratings averaged over participants, plotted over levels of TMS intensity separately for stimuli presented on the lower left (LL) and upper right (UR) with pulses applied at SOA $+90 \mathrm{~ms}$. It appears that, analogously to the objective task performance results, there was a specific effect of Int for LL trials. Statistically, indeed there was an interaction between StimPos and Int $(\mathrm{F}=11.6$; $\mathrm{P}<0.001)$, and follow-up tests revealed a significant effect of Int for $\mathrm{LL}(\mathrm{F}=18.9$; $\mathrm{P}<0.001)$ that was linear $(\mathrm{F}=23.4 ; \mathrm{P}=0.001)$ and not for $\mathrm{UR}(\mathrm{P}=0.652)$.

Also in the other time windows, the effects of TMS on subjective visibility ratings followed closely the patterns of results on behavioral discrimination performance. For post-stimulus SOA $+120 \mathrm{~ms}$ (Figure 2D), the interaction between StimPos and Int was significant $(\mathrm{F}=3.6 ; \mathrm{P}<0.05)$ (even though this interaction did not reach significance for the objective measure, see above). Again, there was a significant effect of Int for $\mathrm{LL}(\mathrm{F}=5.2 ; \mathrm{P}<0.01)$ that was linear $(\mathrm{F}=9.9 ; \mathrm{P}=0.01)$, but not for $\mathrm{UR}$ $(\mathrm{P}=0.708)$. Also for the pre-stimulus windows, there was a significant interaction StimPos $\mathrm{x}$ Int for SOA $-20 \mathrm{~ms}(\mathrm{~F}=12.1 ; \mathrm{P}<0.001)$, and a significant effect of Int for $\mathrm{LL}$ $(\mathrm{F}=14.7 ; \mathrm{P}<0.001)$ that was linear $(\mathrm{F}=19.9 ; \mathrm{P}=0.001)$ but no effect for $\mathrm{UR}(\mathrm{P}=0.600)$. In contrast to behavioral performance, for visibility ratings in SOA $-50 \mathrm{~ms}$ there was no interaction StimPos $\mathrm{x}$ Int $(\mathrm{P}=0.146)$, but a main effect of Int $(\mathrm{F}=11.0 ; \mathrm{P}<0.001)$.

\section{Objective versus subjective results}

By and large, the patterns of results for visibility ratings shown in Figure 2 strongly resemble those obtained for objective discrimination performance shown in Figure 1, and the statistical results suggest a strong correspondence. The only differences were the interaction StimPos x Int for SOA -50 ms (significant only for objective measure), and the interaction StimPos $\mathrm{x}$ Int for SOA $+120 \mathrm{~ms}$ (significant only for subjective measure). However, to directly compare whether the patterns of results over StimPos, SOAs, and Int, differed between the two measures of vision, we standardized the results (see Methods) and plotted them together in Figure 3.

The data on hit rate and visibility are thus shown together in standardized 'space', all scales are equal. There does not seem to be a clear dissociation between the two for any SOA or stimulus position. A RM-ANOVA with factors MV (measure of vision: hit rate or visibility), StimPos, SOA, and Int, did not reveal a four-way interaction between these factors $(\mathrm{P}=0.929)$, or other interactions suggesting that TMS affected visibility or hit rate specifically. 


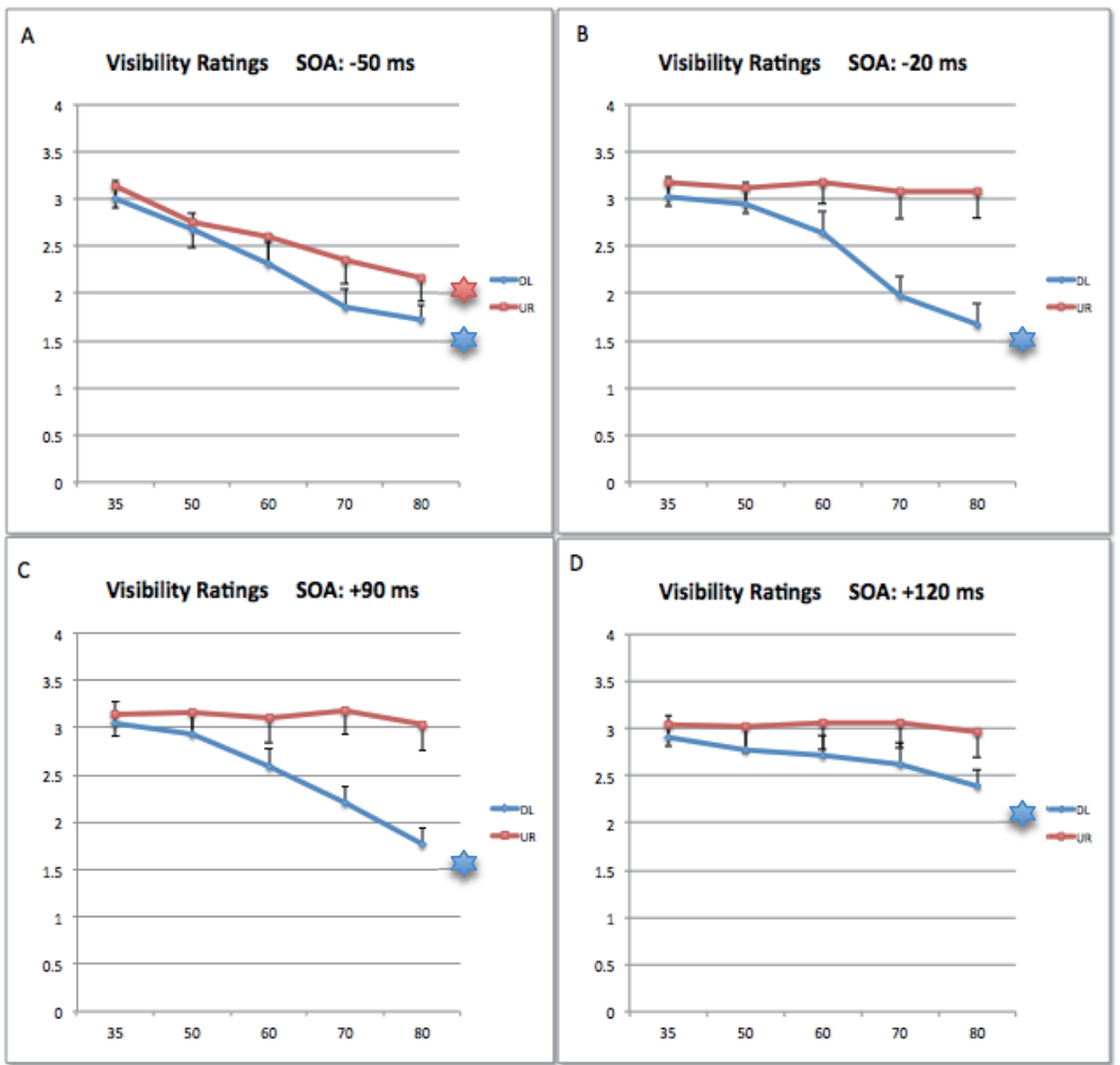

Figure 2: Results for Visibility Ratings

A. Plotted over TMS stimulation intensity (percentage of machine output on horizontal axis), separately for LL (blue lines) and UR (red lines), are the mean visibility ratings for the SOA $50 \mathrm{~ms}$. B. Same as in A but for SOA -20 ms. C. Same as in A but for SOA $+90 \mathrm{~ms}$. D. Same as in A but for SOA +120 ms. All error bars are standard error of the mean (SEM). Stars indicate that there was a significant effect of TMS intensity on hit rate (blue for targets LL, red for targets UR).

\section{Discussion}

In the current work, we addressed two main questions concerning TMS masking effects on subjective and objective measures of visual processing. First, we evaluated whether TMS masking specifically affects 'objective' visual discrimination performance, or 'subjective' stimulus orientation visibility ratings, for two pre-stimulus and for two post-stimulus time windows, for a range of stimulation intensities. Second, we aimed to establish the validity of previously reported pre-stimulus masking windows, stimulating occipital cortex at either $50 \mathrm{~ms}$, or $20 \mathrm{~ms}$, before onset of visual stimuli. We address both questions in turn. 


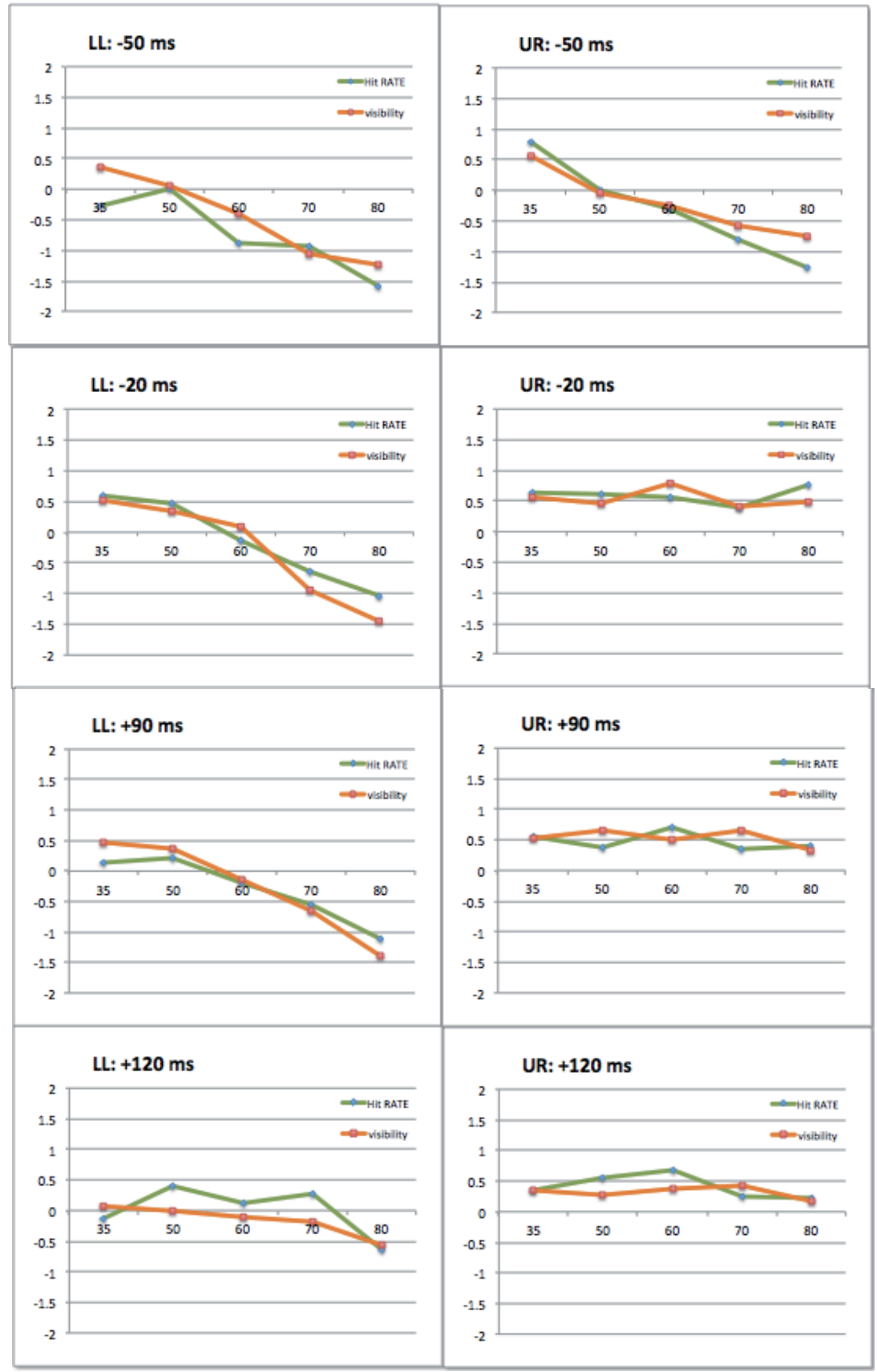

Figure 3: Standardized Results for Hit Rate and Visibility

Per stimulus location and SOA, the effect of intensity is plotted separately for Hit Rate (in green) and Visibility ratings (in orange). The vertical axes are normalized data (see Methods) and are identical in all plots, to compare these patterns to each other and between SOAs and stimulus position. By and large, there appear to be no dissociation between the effects of TMS on both measures of vision. 
No selective disruption of subjective visibility or visual task performance.

In our previous work (de Graaf, Cornelsen, Jacobs, \& Sack, 2011), we evaluated the selectivity of TMS masking effects for an objective and a subjective measure of vision for the classical masking window ( $+90 \mathrm{~ms})$ and one pre-stimulus masking window ( -25 $\mathrm{ms}$ ). We measured a range of TMS intensities, because previous research had been inconsistent in reports of selective TMS effects (e.g. Boyer, Harrison, \& Ro, 2005; Koivisto, Mantyla, \& Silvanto, 2010). Our reasoning was that perhaps there was a small range of stimulation intensity in which the dissociation between disruption of both measures should be apparent. We did not find this to be the case. Here, we added poststimulus masking window $+120 \mathrm{~ms}$. This was of interest in light of new considerations in a TMS context that the first phase of the traditional masking window reflects feedforward activity but soon afterwards (local) recurrent activity could take place in early visual cortex (Boehler, Schoenfeld, Heinze, \& Hopf, 2008; de Graaf, Goebel, \& Sack, 2012; Foxe \& Simpson, 2002; Koivisto, Henriksson, Revonsuo, \& Railo, 2012; Koivisto, Railo, \& Salminen-Vaparanta, 2011), that might be responsible for conscious perception of stimuli (Koivisto, Railo, \& Salminen-Vaparanta, 2011; Lamme, 2001, 2006).

Thus, if in the previous study we disrupted the feedforward phase, it would be of interest to also disrupt the later phase in a similar paradigm (M. Koivisto, personal communication). Perhaps no dissociation between TMS effects on 'unconscious' (objective) and 'conscious' (subjective) measures should be expected for the $+90 \mathrm{~ms}$ window, but a dissociation would occur for the later $+120 \mathrm{~ms}$ window. Koivisto et al. (2011) used TMS to mask either symbol stimuli or orientation stimuli, measuring effects on objective and subjective vision, and found a specific disruption of visual awareness only for orientation stimuli only at $120 \mathrm{~ms}$. We could here not replicate this dissociation over a range of intensities. However, we could previously show that the stimulus used can affect the TMS masking curve (de Graaf, Goebel, \& Sack, 2012), and we did not measure other post-stimulus time windows than strictly fixed $+90 \mathrm{~ms}$ and $+120 \mathrm{~ms}$. This null result should thus be taken with caution (de Graaf \& Sack, 2011).

\section{The specificity of TMS masking effects for pre-stimulus SOAs}

As outlined in the Introduction, previous work has reported the existence of TMS effects around or before visual stimulus onset (Corthout, Hallett, \& Cowey, 2003; Corthout, Uttl, Juan, Hallett, \& Cowey, 2000; Corthout, Uttl, Walsh, Hallett, \& Cowey, 1999; Corthout, Uttl, Ziemann, Cowey, \& Hallett, 1999; de Graaf, Cornelsen, Jacobs, \& Sack, 2011; Jacobs, De Graaf, Goebel, \& Sack, 2012; Jacobs, Goebel, \& Sack, 2012; Laycock, Crewther, Fitzgerald, \& Crewther, 2007; Stevens, McGraw, Ledgeway, \& Schluppeck, 2009), although the specificity of these effects is difficult to determine. For post-stimulus TMS pulses, at least one can be sure that the visual sensory information has successfully 'entered the brain', as the stimulus has come and gone well before the TMS pulse with its accompanying non-neural side effects has been administered. These non-specific effects of TMS can take various forms, including potentially induced eye blinks (Corthout, Uttl, Juan, Hallett, \& Cowey, 2000; Corthout, Uttl, Ziemann, Cowey, 
\& Hallett, 1999; Sack, Kohler, Linden, Goebel, \& Muckli, 2006), the auditory click or sensory stimulation on the scalp possibly leading to multisensory interactions (Meylan \& Murray, 2007), or cognitive effects such as attentional blinks (Shapiro, Raymond, \& Arnell, 1997). Previously, we could show that eye blinks are not the (sole) cause of prestimulus TMS masking effects (Jacobs, Goebel, \& Sack, 2012). Also, we recently showed that pre-stimulus TMS effects around $-50 \mathrm{~ms}$ did occur for occipital TMS, but not for SHAM or VERTEX stimulation (Jacobs, De Graaf, Goebel, \& Sack, 2012). As VERTEX stimulation has sensory effects on the scalp, and both SHAM and VERTEX stimulation have auditory effects, this argues against multisensory mechanisms or cognitive mechanisms such as the attentional blink as explanations of the pre-stimulus masking window. Unfortunately, VERTEX stimulation in that experiment involved a different sample of participants. Yet, there was no indication of any disruptive effects.

In the current work, we employed an additional form of control for TMS masking specificity, by presenting stimuli at either the targeted visual field location or a control location. Interestingly, we found a dissociation between results for the two prestimulus windows tested: $-50 \mathrm{~ms}$ and $-20 \mathrm{~ms}$. These windows were selected based on early work by Corthout and colleagues, which suggested that there might be two separate pre-stimulus SOAs in which TMS could disrupt vision (see references above). For the $-20 \mathrm{~ms}$ window, we found a clear pattern of increasing visual disruption with increasing TMS intensity that was specific to the targeted visual field location. This strongly supports our notion that this SOA constitutes a 'genuine' TMS masking effect (see previous Chapter and de Graaf et al., 2011, for considerations on its underlying mechanism).

For $-50 \mathrm{~ms}$, there was also a 'masking effect', but it was not specific (see also Corthout, Uttl, Ziemann, Cowey, \& Hallett, 1999). Since we did not measure eye blinks, we cannot exclude that the effect on the non-targeted visual field location in this case was due to induced blinking. Yet, as blinking does not occur on all trials (Jacobs, Goebel, \& Sack, 2012), some additional masking effects should be specific. For the objective task, there was indeed a significant interaction between stimulus location and intensity, but this was not replicated by the subjective visibility ratings and in any case TMS intensity significantly affected vision in both visual field locations. We should consider seriously how similar the effects were for both visual field locations, and consider alternative scenarios. In this regard, our data do not allow much interpretation. Of course, non-specific TMS masking effects do not imply 'non-neural' effects. Even if the masking effect is not retinotopic, it could still be due to direct neural mechanisms induced by the TMS pulse. To further elucidate this, three further approaches to the -50 ms window would be valuable. First, future work should implement both control stimulus locations and control TMS sites, preferably close to occipital cortex, in the same subject sample. Second, online brain imaging, such as EEG, might be applied to probe the mechanisms underlying the pre-stimulus masking effect. Third, different stimuli should be used. Previous work (Jacobs, De Graaf, Goebel, \& Sack, 2012; Jacobs, Goebel, \& Sack, 2012) revealed TMS masking around $-50 \mathrm{~ms}$, but not at -20 $\mathrm{ms}$, using symbol stimuli (arrows). Here, the -20 ms masking effect was clear using orientation stimuli. Exploring this dissociation further might be useful to better understand the underlying mechanisms. 


\section{Comparison to previous findings}

The current experiment in part replicated the results of the previous chapter, but did not reproduce completely those findings. We did not here find a different pattern of stimulation intensity, whereas previously we obtained for the pre-stimulus masking window a linear effect of intensity on masking, and a quadratic masking effect for the post-stimulus window (i.e. masking after a certain 'cut-off'). However, in the current experiment we did not implement the same broad range of intensities so perhaps this design was not sensitive enough to accurately detect these trends. The second difference is that previously we found the pre-stimulus window to be 'more sensitive': lower stimulation intensities were required to obtain masking effects. This was not replicated here. The pre-stimulus SOA was slightly changed (-25 ms vs. $-20 \mathrm{~ms})$, but given the exploratory nature of the previous work and the larger sample size here, it could be that the pre-stimulus masking effect is in fact not more sensitive than the classical masking effect around $90 \mathrm{~ms}$. The main findings of the previous work were, however, strongly confirmed. We here replicated strong masking effects of TMS pulses applied before visual stimulus onset, that scaled linearly with TMS intensity, and were (here shown to be) specific to the targeted visual field location.

\section{Conclusion}

TMS can disrupt visual processing not only in the classical masking window, but also when applied before the onset of visual stimuli. Both masking effects are highly similar if not identical for objective and subjective measures of vision. 



\section{II.5}

\section{The Chronometry of Visual Perception:}

review of occipital TMS masking

studies

Related publication(s):

De Graaf, TA, Koivisto, M*, Jacobs, C, Sack, AT (in preparation). The chronometry of visual perception: a review of occipital TMS masking studies

* Mika Koivisto

1) Centre for Cognitive Neuroscience and Department of Psychology, University of Turku, Turku, Finland 


\begin{abstract}
Transcranial Magnetic Stimulation (TMS) continues to deliver on its promise as a research tool. In this review article we focus on the application of TMS to early visual cortex $(\mathrm{V} 1, \mathrm{~V} 2, \mathrm{~V} 3)$ in studies of visual perception and visual awareness. Depending on the asynchrony between visual stimulus onset and TMS pulse (SOA), TMS can suppress visual perception, allowing one to track the time course of functional relevance (chronometry) of early visual cortex for vision. This procedure has revealed multiple masking effects ('dips'), some consistently $(\sim+100 \mathrm{~ms}$ SOA) but others less so $(\sim-50 \mathrm{~ms}, \sim-20 \mathrm{~ms}, \sim+30 \mathrm{~ms}, \sim+200 \mathrm{~ms} \mathrm{SOA})$. We review the state of TMS masking research, focusing on the evidence for these multiple dips, the relevance of several experimental parameters to the obtained 'masking curve', and the use of multiple measures of visual processing (subjective measures of awareness, objective discrimination tasks, priming effects). Lastly, we consider possible future directions for this field.
\end{abstract}




\section{Introduction}

Amassian and colleagues effectively started a new scientific field when they used magnetic pulses to disrupt human vision in 1989 (Amassian et al., 1989). Transcranial magnetic stimulation (TMS) pulses are rapidly changing magnetic fields (of up to $\sim 2$ Tesla field strength), elicited by a coil attached to a strong capacitator, which penetrate the skull and induce an electric field in underlying brain tissue. The strength and focality of the induced field depend on coil characteristics. Round coils consist of a single wired loop and induce a large field around their rim. Today, figure- 8 or butterfly coils are used more often, since their two overlapping coils result in a relatively focal peak magnetic field. Aside from single TMS pulses, short bursts of 2, 3 or even more pulses can be applied to briefly disrupt neuronal processing (for basic TMS methodology see Wassermann et al., 2008). But the more pulses that are administered per trial, the less temporally specific the experimental paradigm becomes. And indeed, the potential for temporal specificity is one of the key values of TMS as a research tool. The mechanical process underlying a single TMS pulse takes less than a millisecond, but its neuronal effects can linger for seconds (Moliadze, Zhao, Eysel, \& Funke, 2003). Nevertheless, when applied to more robust neuronal populations, i.e. brain regions, TMS is considered a useful tool to probe chronometrically the functional relevance of targeted areas in the intact human brain (Pascual-Leone, Walsh, \& Rothwell, 2000). In the work that we review here, that targeted area is the human early visual cortex (EVC).

\section{TMS masking}

TMS was first applied to human motor cortex to successfully elicit muscle contractions (Barker, Jalinous, \& Freeston, 1985). The work by Amassian et al. (1989) four years later constituted a significant advance for at least three reasons. First, TMS was applied to probe a brain region other than motor cortex, namely occipital cortex, with very clear behavioral/perceptual effects. Second, their study was a dramatic demonstration of the chronometric potential of TMS as a research tool: they used the brief disruptive effects of the magnetic pulses to track the role of occipital cortex in visual perception as a function of time. Third, due to this, something fundamental was added to our knowledge about human vision, since no other method could show when a brain region was necessary, not only involved, at a particular time point and for a particular task.

In this seminal experiment, TMS was applied to posterior occipital cortex where visual information first reaches the human neocortex (Amassian et al., 1989). Visual information consisted of three letters, presented to subjects on a computer screen. The task was to report as many of the letters as possible. Occipital TMS pulses were applied at various stimulus onset asynchronies (SOAs) in relation to the visual stimulus onset. This original TMS masking paradigm was thus suited to answer two questions: 1) is early visual cortex functionally relevant for visual task performance? and 2) at which precise time points (SOAs) is this the case? 
Results showed that no letters at all were correctly identified when pulses were applied at 80 or $100 \mathrm{~ms}$ SOA. Nearly all letters were correctly identified when pulses were applied before $60 \mathrm{~ms}$, or after $140 \mathrm{~ms}$. Performance was intermediate in the time windows 60 and $120 \mathrm{~ms}$. Thus, a clear TMS masking curve was demonstrated. But in their original and short research report, Amassian and colleagues (1989) actually addressed a number of additional questions.

Firstly, they asked whether or not the masking effect is neural in origin. The magnetic stimulation also elicits muscle contractions in the neck, and makes a loud 'clicking' sound. These factors may affect attention, which in turn may affect performance in visual tasks. Moreover, a startle response to either of these factors could elicit eyeblinks, which may affect visual performance. Amassian and colleagues (1989) immediately showed that such non-neural TMS effects could not fully explain the effects, since moving the magnetic coil sideways or upwards affected the specific letters that were recognized/reported in exactly the fashion that we should expect based on known retinotopic organization of visual cortex. While this demonstrated a neural disruption, this did not prove that non-neural effects have no influence on the masking curve: each new TMS masking experiment must continue to control for possible nonneural TMS effects.

The original article also addressed the questions 1) precise brain regions/processes are affected by the TMS pulses? 2) which stage of visual processing is affected by TMS? 3) can and do the pulses affect conscious and/or unconscious vision selectively? In addition and relation to these questions, more recent TMS masking studies have focused on the issue 4) is the 'classical' TMS masking effect around $100 \mathrm{~ms}$ SOA the only time point at which early visual cortex is functionally relevant for vision?

These questions were prescient, as they form the foundations of the TMS masking field today. Here, we aim to provide an overview of what has been added to this pioneering work in the last 20 years. We will focus exclusively on TMS masking studies targeting early visual cortex, and work related to this. The last TMS masking reviews that we are familiar with were published in 2007 by Thomas Kammer (Kammer, 2007, 2008). After that, interesting new work has appeared, clarifying some issues but raising new ones as well. It is time for an update.

\section{The multiple regions stimulated in TMS masking}

Visual TMS masking studies have used several different procedures for targeting the pulses on visual cortex or more specifically on the primary visual cortex (i.e., V1 or striate cortex). The early studies used an anatomical landmark method, for example by placing the TMS coil 1-3 cm above the inion (e.g. Amassian et al., 1989; Corthout, Hallett, \& Cowey, 2002, 2003; Corthout, Uttl, Juan, Hallett, \& Cowey, 2000; Corthout, Uttl, Walsh, Hallett, \& Cowey, 1999) or over occipital electrode sites according to the international 10/20-system (e.g. Beckers \& Homberg, 1991). Another procedure is the hunting method. Here the location of the coil is systematically moved, starting for example from $2 \mathrm{~cm}$ above the inion, until a location where TMS pulses produce visual 
suppression is found. A variant of the hunting method is the phosphene localization method (e.g. de Graaf, Goebel, \& Sack, 2012; Silvanto, Cowey, Lavie, \& Walsh, 2005). It makes use of phosphenes, flashes of light, which TMS applied over visual cortex elicits in many (but not all) participants. In this method, the coil is moved systematically over the posterior cortex (starting e.g. $2 \mathrm{~cm}$ above the inion) until a position where phosphenes in a specific retinotopic location are found. This method is based on the assumption that the neural populations which produce phosphenes in a given location of the visual field are the same neural populations which are responsible for coding the visual stimulus that is presented in the same retinotopic area (e.g. Kammer, Puls, Erb, \& Grodd, 2005).

These procedures successfully elicited masking effects. Yet the informational value of these effects would be vastly increased by knowledge of the precise brain areas that were disrupted. What is the exact cortical area that is stimulated with these procedures? Recent studies using fMRI-based mapping of visual cortex in individual participants in combination with modeling of the TMS-induced electric field in brain have examined this question (Salminen-Vaparanta, Noreika, Revonsuo, Koivisto, \& Vanni, 2012; Thielscher, Reichenbach, Ugurbil, \& Uludag, 2010). Salminen-Vaparanta et al. (2012) studied the stimulation site in the anatomical landmark method by making use of fMRI based retinotopic maps of V1 and V2 subareas and modeling of the TMSinduced electric field in the retinotopic subareas of V1 and V2. They found that when the TMS coil was positioned $2 \mathrm{~cm}$ above the inion, both V1 and V2 were stimulated but there was a great deal of variation between participants. On average, the dorsal V2 was the most affected functional area and the lower visual field areas received the strongest stimulation. For the hunting and phosphene localization procedures, Thielscher et al. (2010) found that V2d was the area most likely responsible for visual suppression of the stimuli in the lower right visual field. In addition, they observed that isolated V3 stimulation produced visual suppression. The modeling of the TMS-induced electric field for two of the participants in Koivisto et al. (2011) also showed clearly that the stimulation location defined by the hunting method for a stimulus in the left or right lower visual field ( $2 \mathrm{~cm}$ above and $2 \mathrm{~cm}$ lateral from inion) resulted in stronger electric field in the corresponding retinotopic area in V2/V3 border than in V1.

Even when explicitly aiming to selectively stimulate V1, using neuronavigation to directly target individual functionally-defined V1, modeling of the electric field showed that TMS-induced electric field was stronger in V2 than in V1 for about half of the participants in Salminen-Vaparanta et al. (2012). While it thus appears to be difficult to selectively disrupt V1, selective stimulation of V2 has been confirmed to induce visual suppression (Salminen-Vaparanta, Koivisto, Noreika, Vanni, \& Revonsuo, 2012). Thus, although some have argued on functional/behavioral grounds that V1 is the stimulated region in at least some experiments (e.g. Juan \& Walsh, 2003), these modeling results converge in suggesting that the traditional anatomical landmark method or hunting methods lead to equally strong or even stronger stimulation of V2 (perhaps also V3) than V1. Therefore it would be a mistake to attribute the visual suppression effects obtained with these methods to the effects of TMS on V1 only, even if this has conventionally been considered the target(ed) region. Of course, especially with closely neighboring and connected functional regions it is impossible to say 
precisely how the induced electric field translates to neural activity in those regions. For example, stimulation of V2/V3 might still affect V1 through anatomical connections (Kammer, Puls, Erb, \& Grodd, 2005). Yet, it seems safe to conclude for the moment that multiple early visual regions are functionally relevant for visual perception. In the remainder of this review we refer to 'early visual cortex' (EVC) as the TMS-disrupted site in masking studies.

\section{The multiple dips in the masking curve}

Amassian et al. (1989) found one large dip in the TMS masking curve. By 'dip' we mean a disruption of visual task performance (in figures generally visualized as a downward deflection from baseline of task performance or visibility report - hence 'dip'). The dip found by Amassian et al. (1989) we have referred to as the 'classical dip' (de Graaf, Cornelsen, Jacobs, \& Sack, 2011; de Graaf, Goebel, \& Sack, 2012; de Graaf, Herring, \& Sack, 2011), because this masking effect around 100ms SOA has been replicated in every TMS masking study that we know of. Clearly, something fundamental happens in early visual cortex around $+100 \mathrm{~ms}$, and it is very susceptible to TMS pulses. But the exact timing of the dip around $+100 \mathrm{~ms}$ varies between experiments. Moreover, one should question whether this is the only time point at which early visual cortex is functionally relevant for conscious vision.

\section{The feedforward-feedback hypothesis}

Visual information passes early visual cortex once, on its way to the rest of the brain along several pathways (e.g. DeYoe, Felleman, Van Essen, \& McClendon, 1994; Felleman, Burkhalter, \& Van Essen, 1997). This first pass-through can be referred to as a 'feedforward sweep'. But according to various psychological and neurobiological models (Bullier, 2001; Lamme, 2001; Lamme, Super, Landman, Roelfsema, \& Spekreijse, 2000; Pollen, 2003), a feedback signal to early visual cortex might be functionally relevant for vision as well. This recurrent signal could be important to 'check' processing results with the input (Bullier, 2001; Di Lollo, Enns, \& Rensink, 2000), to fill in the finer details of the visual image (Hochstein \& Ahissar, 2002), or be responsible for visual awareness of the stimulus (Lamme, 2001; Lamme, Super, Landman, Roelfsema, \& Spekreijse, 2000). Whichever particular theory on the role of recurrent processing is correct, a 'feedforward-feedback hypothesis' might be considered to predict not one, but at least two dips in the TMS masking curve. In what follows directly below, we consider studies using static visual stimuli.

\section{The $\sim 30$ ms dip}

Corthout and colleagues (Corthout, Hallett, \& Cowey, 2002, 2003; Corthout, Uttl, Walsh, Hallett, \& Cowey, 1999; Corthout, Uttl, Ziemann, Cowey, \& Hallett, 1999) 
found that TMS pulses at 20-40 ms have an effect on visual task performance. This seems an interesting yet odd dip. Based on visual evoked potential (VEP) studies (e.g. Di Russo, Martinez, Sereno, Pitzalis, \& Hillyard, 2002; Vanni et al., 2004), it has often been assumed that visual inputs do not arrive at EVC until around 50-60 ms poststimulus. However, invasive recordings in monkeys (Celebrini, Thorpe, Trotter, \& Imbert, 1993; Cowey, 1964; Knierim \& van Essen, 1992) and humans (Wilson, Babb, Halgren, \& Crandall, 1983) indicate that the earliest activity in V1 does start before this. And independent evidence suggests that around $100 \mathrm{~ms}$ SOA, recurrent activity to V1 can already take place (see below). Lamme and colleagues (Lamme \& Roelfsema, 2000; Lamme, Super, \& Spekreijse, 1998) moreover provided evidence that two distinct periods of activity in V1 may occur, of which the first could be as early as $40 \mathrm{~ms}$. So, hypothetically, a first masking dip at around 20-40 ms could reflect a feedforward stage of visual processing, while the classical 100 ms masking dip could reflect feedback processing.

On the other hand, there do not seem to be many replications of this dip. That would seem strange if 30-40 ms TMS pulses disturb an automatic feedforward process: ALL information is ALWAYS fed forward, so disruption of feedforward activity should be an effect that is found relatively easily and without much dependence on the visual stimuli or other experimental parameters. Kammer (2008) reviewed the evidence for the 'early' dip around $30-40 \mathrm{~ms}$ and found it inconclusive. He speculated that perhaps inter-individual differences in (functional) anatomy could explain why only sometimes the early dip is found. Perhaps stimulation of precisely V1 (rather than V2/V3) might be necessary for the early dip and so the early dip could only be found in subjects whose primary visual cortex lies relatively close to the surface. But a recent experiment targeting specifically V1 also failed to reproduce the $30-40 \mathrm{~ms}$ dip (Salminen-Vaparanta, Noreika, Revonsuo, Koivisto, \& Vanni, 2012).

Paulus et al. (1999) found a TMS masking effect at 15-45 ms that was separate from a second dip at the classical window around $100 \mathrm{~ms}$. They found this early dip, however, only for achromatic stimuli, and not chromatic stimuli, in the same subjects. They interpreted their data in relation to magnocellular and parvocellular pathways, noting that both pathways are involved in achromatic stimuli, but only the parvocellular pathway is involved in chromatic stimuli. Magnocellular signals are known to be transmitted faster through the early visual areas than parvocellular signals (e.g. Nowak, Munk, Girard, \& Bullier, 1995). Maybe the early dip could thus be a "magno-dip" and the classical dip a "parvo-dip"? Or perhaps the early dip is only found when stimuli are specifically tuned to massively involve the magnocellular pathway. Corthout and colleagues found behavioral impairment around 20-40 ms several times (Corthout, Hallett, \& Cowey, 2002, 2003; Corthout, Uttl, Walsh, Hallett, \& Cowey, 1999; Corthout, Uttl, Ziemann, Cowey, \& Hallett, 1999), but not always as a 'separate' dip (i.e. often 'fused' with other dips). Paulus et al. (1999) found it, and Kammer et al. (2003) found it in one subject out of three. We have previously tested for it and failed to replicate (de Graaf, Herring, \& Sack, 2011; Jacobs, Goebel, \& Sack, 2012) systematically. All the same, null results here are difficult to generalize (de Graaf \& Sack, 2011) and positive results must be duly credited. 
In (intermediate) conclusion, the $30-40 \mathrm{~ms}$ dip is inconsistent, elusive, and not straightforward to interpret. We will see below (see The 'negative' dips) that TMS pulses applied prior to stimulus presentation also affect visual performance, showing that TMS can affect visual processing also when applied before online processing of the visual stimulus has not yet begun. If that is possible, then the lack of straightforward interpretation of a $30-40 \mathrm{~ms}$ dip should not refrain us from investigating this potential dip further.

\section{The $\sim 200 \mathrm{~ms}$ dip}

Consistent with the feedforward-feedback hypothesis are reports of a TMS masking dip AFTER the classical masking window around $100 \mathrm{~ms}$ : such findings lend themselves to interpretation of the classical dip as a feedforward stage of processing while the later dip could reflect feedback processing.

Recently, a second masking dip at $220 \mathrm{~ms}$ SOA, clearly separated from the classical dip, was reported (Camprodon, Zohary, Brodbeck, \& Pascual-Leone, 2010). Subjects performed forced-choice discrimination between natural images of birds and large mammals. This essentially constitutes a categorization task, arguably 'higherorder' than for example orientation discrimination tasks. Also Koivisto et al. (2011) used stationary stimuli to lend support to the feedforward-feedback hypothesis. Using a task of categorizing visual natural scenes (animals vs. nonanimals), they targeted EVC and lateral-occipital cortex (LO: a higher-order ventral stream region). EVC was functionally relevant (the classical dip) before LO, and still functionally relevant after LO started around $150 \mathrm{~ms}$ to become functionally relevant. Although two separate EVC dips were not observed, the pattern of results was not in line with a fully feedforward account, which cannot explain why early visual cortex should be functionally relevant after visual inputs have already reached LO. Previously, Heinen et al. (2005) also found two dips, one (just) within the classical window starting at $130 \mathrm{~ms}$, one quite late starting at $250 \mathrm{~ms}$. Their study focused on figure-ground segregation processes, and their rather late 'classical' effect from 130 to $160 \mathrm{~ms}$ is perhaps not so straightforwardly connected to TMS masking effects using more generic visual stimuli and tasks.

But these studies suggest that EVC may be functionally relevant at this late stage when more 'complex', or 'higher-order' visual processes are required. In support of this conjecture, Juan and Walsh (2003) found that double-pulse TMS over EVC impaired visual search of features at $80 / 120 \mathrm{~ms}$, whereas search of feature conjunctions was impaired by TMS applied 200/240 ms after stimulus onset. Dugué et al. (2011) found that attention-demanding serial visual search was impaired when TMS was applied over early visual cortex $300 \mathrm{~ms}$ after the onset of the stimulus array, whereas TMS had no effect on more automatic parallel search. Koivisto and Silvanto (2012) extended these findings by suggesting that the late V1/V2 activity period after $200 \mathrm{~ms}$ does not necessarily reflect the requirement of serial visual search through a complex display but generalizes also to a situation that is more specific to feature binding.

Attempts to replicate the late $\sim 200$ ms masking dip with simple forms or other simple static stimuli have not appeared successful so far (Jacobs, De Graaf, Goebel, \& 
Sack, 2012; Jacobs, Goebel, \& Sack, 2012; Koivisto, Henriksson, Revonsuo, \& Railo, 2012; Railo \& Koivisto, 2012). For example, a recent study by our group applied single TMS pulses across the identical post-stimulus temporal range measured by Camprodon et al. and with identical resolution, during visual discrimination of symbolic arrow stimuli (Jacobs, De Graaf, Goebel, \& Sack, 2012). The classic masking dip was clear, but no TMS effects after $120 \mathrm{~ms}$ were observed. Thus, it seems that the late dip can be produced only with rather complex stimuli and/or tasks that require figure-ground segregation (Camprodon, Zohary, Brodbeck, \& Pascual-Leone, 2010; Heinen, Jolij, \& Lamme, 2005) or feature binding/conjunction search (Dugue, Marque, \& VanRullen, 2011; Juan \& Walsh, 2003; Koivisto \& Silvanto, 2012).

While this postulation does lend support to the feedforward-feedback hypothesis, it also suggests that the feedforward-feedback framework may need to be refined. It has been suggested that much processing occurs within $100 \mathrm{~ms}$ from stimulus onset, involving perhaps multiple loops of feedforward-feedback (e.g. Bullier, 2001; Corthout, Hallett, \& Cowey, 2003; Foxe \& Simpson, 2002; Juan \& Walsh, 2003). Rather than trying to decide whether the classical dip is either feedforward or feedback, and which secondary dips then constitute the alternative, it may be more useful to consider whether there are not multiple stages at which EVC is functionally relevant and to begin probing the conditions under which these stages appear in TMS masking contexts. As a case in point, a very recent study showed that when two peripheral stimuli had to be compared, even though TMS pulses targeted foveal cortex, the contribution of EVC was necessary for accurate performance as late as $350-400 \mathrm{~ms}$ (Chambers, Allen, Maizey, \& Williams, 2012). In conclusion, EVC may be crucial in various stages of visual processing over hundreds of milliseconds, depending on stimulus and task parameters.

\section{The 'negative' dips}

When occipital TMS pulses are applied PRIOR to the visual stimulus onset (i.e., at negative SOAs), effects on visual task performance have also been reported. Corthout et al. (1999; Corthout, Uttl, Ziemann, Cowey, \& Hallett, 1999) found that TMS pulses prior to visual stimuli could impair performance. In subsequent work, Corthout discriminated two pre-stimulus (i.e., negative) dips, which they dubbed "dip0" and "dipX" (Corthout, Uttl, Juan, Hallett, \& Cowey, 2000). Corthout et al. (2003) reported these dips to be separable. Dip0 was around $\sim 50 \mathrm{~ms}$, dipX was around $\sim-10 \mathrm{~ms}$.

The conceptual problem with negative dips is twofold. First, if visual inputs will arrive to occipital cortex tens of milliseconds after the TMS pulse, how can the pulse disrupt vision? Second, eye blinks with various degrees of severity can be induced by occipital TMS, for instance as a startle-reflex to muscle contractions or the loud clicking noise. Eye blinks during or around stimulus presentation would obviously confound the data.

The potential contribution of TMS-induced eye blinks to the negative dip has been examined in three ways. First, based on the original logic from Amassian et al. 
(1989), it is possible to test whether or not the negative dip is retinotopic. For the $\sim-50$ ms negative dip, this did not seem to be the case (Corthout, Hallett, \& Cowey, 2003). Second, one may study when eye blinks interfere with visual processing, and how long it takes for an eye blink to be elicited by a TMS pulse. Stimulation of the facial nerve (resulting in reflex blinking) could affect visual discrimination of letters presented within an SOA corresponding to dip0 ( $\sim 50 \mathrm{~ms})$ but not dipX ( $\sim 10 \mathrm{~ms})$ (Amassian et al., 1998). Eye-tracking studies during occipital TMS also suggest that blinking could principally explain the $-50 \mathrm{~ms}$ masking effect (measuring the latency of 'pupilcovering' after a pulse) (Corthout, Hallett, \& Cowey, 2011; Corthout, Uttl, Juan, Hallett, \& Cowey, 2000). From this, Corthout et al. (2011) recently concluded "that dip0 is almost certainly caused by pupil covering and that dipX is almost certainly not caused by pupil covering". Of course, even if eye blinks do contribute to a masking dip this does not exclude the possibility of a concurrent neural effect. Third, eye blinks can be measured and simply eliminated from the data to evaluate whether visual suppression still takes place at negative SOAs. Our lab recently replicated the negative dip at a broad range of SOAs (from -80 to $-40 \mathrm{~ms}$ ) and used electro-oculography (EOG) to measure eye muscle activations during a TMS masking experiment. Removing trials with eye blinks did reduce the average extent of visual suppression by TMS, but negative SOAs still showed significant masking effects (Jacobs, Goebel, \& Sack, 2012). Only one large negative dip was found, which was variable in latency across subjects, so the postulated dip0 and dipX were not separated. But all this work does suggest that at least some pre-stimulus masking (negative dip) is possible. We recently demonstrated, using TMS over a range of TMS intensities at SOAs of $+90 \mathrm{~ms}$ and $25 \mathrm{~ms}$, that the pre-stimulus masking effect can be very sensitive and easily detected using small orientation stimuli (de Graaf, Cornelsen, Jacobs, \& Sack, 2011). In a new study, we moreover showed that these TMS masking effects at an SOA of $-20 \mathrm{~ms}$ are retinotopically specific, while TMS masking at an SOA of $-50 \mathrm{~ms}$ also involved nonspecific effects (previous Chapter).

How can we explain these TMS masking effects? At post-stimulus (positive) SOAs, TMS presumably affects the signal-to-noise ratio of online visual information processing. But a fundamentally different mechanism must occur for pre-stimulus (negative) SOAs. This could have a bearing on a putative $30-40 \mathrm{~ms}$ dip as well. In de Graaf et al. (2011), we suggested that while post-stimulus TMS pulses disrupt the signal-to-noise ratio (SNR), pre-stimulus TMS pulses may put occipital cortex in a particular state that is not conducive to future information processing. We speculated that this may involve a modulation of intrinsic brain oscillations in the visual system's natural frequency band (alpha-band: $\sim 8-12 \mathrm{~Hz}$ ) (Jacobs, De Graaf, Goebel, \& Sack, 2012; Jacobs, Goebel, \& Sack, 2012) since previous work shows that the power (Thut, Nietzel, Brandt, \& Pascual-Leone, 2006) and phase (Busch, Dubois, \& VanRullen, 2009; Mathewson, Gratton, Fabiani, Beck, \& Ro, 2009) of ongoing parieto-occipital alpha oscillations directly determine visual performance (see also Chapter "alpha entrainment"). Other (potentially related/coinciding) neural accounts of the pre-stimulus masking dip that have been suggested are interference with expectation-driven enhanced baseline activity prior to stimulus onset (Laycock, Crewther, Fitzgerald, \& Crewther, 2007) and extension of the TMS effect into lower level (sub)cortical visual 
areas either via recurrent connections (Stevens, McGraw, Ledgeway, \& Schluppeck, 2009) or anthrodromically through feedforward connections (Cowey, 2008). These various explanations are not mutually exclusive, but further work is required to probe their contributions.

\section{The classical dip: $\sim 100 \mathrm{~ms}$}

Fortunately, there is one dip that is clear and uncontested: the classical dip around +100 ms. Amassian et al. (1989) initially supposed that the masking curve delineated the timing of information entering and exiting EVC, an interpretation as elegant as straightforward. Perhaps because of this, the classical masking dip is fairly undifferentiated in the current TMS masking literature. There are a limited number of studies focusing on how various factors (e.g. TMS coil shape, TMS intensity, occipital targeting method, visual stimulus type) affect parameters of the classical dip (e.g. width of the curve, onset of the curve, peak masking latency, recovery of performance). Notable examples include reports of the effect of TMS intensity on width of the masking curve (e.g. Beckers \& Homberg, 1991 : onset latency is earlier with higher intensity), the effect of stimulus luminance on masking onset latency (e.g. Kammer, Puls, Strasburger, Hill, \& Wichmann, 2005): with increasing luminance masking starts at earlier SOAs), or the interplay between occipital gyral/TMS coil orientation (Kammer, Vorwerg, \& Herrnberger, 2007). Recent studies have also indicated that the type of stimulus has an effect on the latency of the classical dip. De Graaf et al. (2012) showed in the same subjects that orientation gratings had an earlier peak masking latency than faces. Koivisto et al. (2011) found with a between-subjects design a longer dip for judgments of symbolic arrow direction than for orientation of bars.

But on reflection, a window spanning $60 \mathrm{~ms}$ to $140 \mathrm{~ms}$ SOA is SO broad, it seems actually implausible that one simple and same process is going on in all the TMS masking studies addressing the classical masking finding. In a recent exploratory study, with a 'sensitive paradigm' including small and peripheral stimuli and relatively low TMS intensity, we saw at least two closely neighboring masking dips within the classical dip, with performance returning to baseline in-between (de Graaf, Herring, \& Sack, 2011). Another example why the $100 \mathrm{~ms}$ dip may be less straightforward than it appears is recent work by Abrahamyan et al. (2011), who showed that when the intensity of TMS is just below phosphene threshold, visual task performance can actually be improved rather than impaired, whereas masking occurs with higher than phosphene threshold intensities. Thus, weak intensity TMS pulses bring the activation of neurons closer to the detection threshold, whereas strong pulses decrease the detection of sensory event, suggesting a sigmoid nonlinearity in the input response functions of the neurons. In sum, our focus on the classical dip as either the feedforward stage or the feedback stage in the context of the feedforward-feedback hypothesis may have been overly simplistic. 


\section{Two dips in motion masking studies}

We have been liberal with our term of the 'feedforward-feedback hypothesis', using it to refer to a collection of theories that have one thing in common: EVC is functionally relevant not only when information first reaches it, but also afterwards, when information feeds back to it from higher-order areas. The most straightforward type of evidence for this scenario would come from a pattern of TMS results in a specific task where 1) early visual cortex is shown to be relevant, 2) a higher-order visual region is shown to be relevant after this, 3) early visual cortex is again shown to be functionally relevant after the functionally relevant window of the higher-order region. To test this particular framework, two visual areas need to be stimulated in the same subjects: early visual cortex and a higher-order visual region that can be targeted by TMS.

One candidate higher-order region for demonstrating the feedforwardfeedbackward cycle between ECV and extrastriate cortex is the human motion area V5, or hMT. Pascual-Leone and Walsh (Pascual-Leone \& Walsh, 2001) used two TMS coils, one targeting hMT and one targeting EVC. TMS over hMT induced moving phosphenes. EVC was then stimulated at various SOAs around the hMT stimulation pulse. Interestingly, a TMS pulse to EVC (below phosphene threshold), when applied some 30-40 ms AFTER the pulse to hMT, abolished the apparent movement of the phosphenes or abolished the moving phosphenes altogether. This indicated that some form of recurrent signals from hMT to early visual cortex were actually necessary for the visual awareness of moving phosphenes to arise. Silvanto et al. (2005) also stimulated hMT, but with a subthreshold pulse (which in isolation did not lead to any, moving or otherwise, phosphenes) and early visual cortex with a suprathreshold pulse. Suprathreshold pulses to early visual cortex in isolation led to stationary phosphenes, but when they were preceded (by some 10-50 ms) by subthreshold hMT stimulation the phosphenes took on hMT characteristics: they became bigger and were moving (Silvanto, Cowey, Lavie, \& Walsh, 2005). Thus, the backprojections could determine the content of the conscious visual experiences.

These studies showed that feedback per se is necessary for moving phosphenes perception, and that feedback per se can codetermine the content of conscious perception. But these results do not yet validate the feedforward-feedback hypothesis for regular visual perception. Silvanto et al. (2005) asked subjects to report awareness of motion dot displays, while applying TMS to EVC or hMT across a range of SOAs. EVC was functionally relevant first, then hMT was functionally relevant but EVC was not, and subsequently, EVC was again functionally relevant in a second, distinct masking dip. In both time windows when EVC was functionally relevant, hMT was not. In this scenario, the first EVC masking dip could represent a feedforward stage, and the second EVC masking dip must represent a feedback stage. hMT does its functionally relevant processing in-between.

It would be valuable to directly relate such motion masking findings to the multiple masking dips reported in stationary masking experiments. Unfortunately, studies with two separate dips in motion perception (Koivisto, Mantyla, \& Silvanto, 2010; Silvanto, Lavie, \& Walsh, 2005) have often triggered the TMS pulses in relation 
to stimulus offset. And since the stimulus durations were determined separately for each individual participant TMS latencies cannot be straightforwardly translated to SOAs. If we do calculate the SOAs for stimuli with an average duration $(67 \mathrm{~ms})$ in Koivisto et al. (2010), the SOA for the early EVC dip was $87 \mathrm{~ms}$ and for the late EVC dip it was 127 ms. The early dip, hypothetically corresponding to feedforward stage, thus falls clearly to the time window of "classical dip", while the later dip, hypothetically corresponding to recurrent feedback, seems to parallel the end part of it. This might suggest that the classical dip cannot be said to reflect one of these stages, but in itself contains several stages.

\section{Multiple measures of vision: conscious and unconscious processing}

Amassian et al. (1989) already noted that sometimes participants were correct in their identification of the presented letters, even though they reported that they did not consciously perceive the letters. "Such reports hint at a dual process, the first registering the letters at an unconscious level and the second rapidly entering consciousness but more susceptible to the MC pulse" (Amassian et al.,1989, p.460). This remark foreshadowed the current interest in the role of EVC in conscious awareness versus unconscious information processing. Only recently have TMS masking studies begun to incorporate multiple measures of visual processing, including direct subjective report of conscious perception, forced-choice behavioural tasks, and (un)conscious priming effects.

Ro et al. (2004) and Boyer et al. (2005) first addressed the issue systematically, optimizing the TMS masking paradigm to simulate 'blindsight'. Blindsight patients have lesions in the primary visual cortex, resulting in scotomas, or conscious blind spots, in their visual field (Weiskrantz, 1996). Even in the absence of conscious perception these patients can relatively accurately process some information about stimuli presented in the blind areas, such as motion direction or the location of stimuli. Ro et al. (2004) showed in normal participants that, when TMS pulses to early visual cortex successfully masked visual stimuli, these stimuli could still affect saccades. Boyer et al. (2005) went a step further, directly assessing the subjective experience of subjects ("did you see the orientation of the bar, yes or no") on every trial, and assessing simultaneously the objective information processing capacity of subjects ("forced-choice guess: was the bar horizontal or vertical?") on every trial. Five subjects performed highly accurately, with up to $90 \%$ correct orientation judgments, even though they reported no conscious percept of the stimuli. The same was found for color judgments (Boyer, Harrison, \& Ro, 2005).

These results seemed to emulate blindsight, suggesting that early visual cortex is necessary for conscious perception but not unconscious processing of orientation and color stimuli. But the experimental paradigm in Boyer et al. (2005) did not test the range of SOAs corresponding to the early latencies in the classical dip. It has been suggested that some additional control conditions would more clearly demonstrate whether discrimination performance without reported awareness was affected by TMS (Koivisto, Mantyla, \& Silvanto, 2010). Indeed, in our own efforts concerning this issue, 
we have been able to replicate the finding that subjects perform above-chance on trials where they do not consciously report seeing the stimulus (de Graaf, Cornelsen, Jacobs, \& Sack, 2011; Jacobs, Goebel, \& Sack, 2012). But we found this to be the case for noTMS trials (not measured in Boyer et al. (2005)) as well, and in one dataset the accuracy on non-perceived trials (trials without reported awareness) with SHAM (placebo) TMS was actually higher than the accuracy on non-perceived trials with real TMS pulses, across a range of SOAs (de Graaf, Cornelsen, Jacobs, \& Sack, 2011). While statistically underpowered, these observations emphasized the need for further control conditions. Without them, 'TMS-induced blindsight results' may be explained by a difference in sensitivity of the two measures of visual processing.

Nevertheless, these reports inspired future studies to incorporate multiple measures of visual processing. We have generally employed two measures of vision, one subjective (e.g. a stimulus visibility rating on a four-point scale) and one objective (e.g. 2-alternative forced-choice - 2AFC). We evaluated across SOAs (de Graaf, Goebel, \& Sack, 2012; de Graaf, Herring, \& Sack, 2011; Jacobs, De Graaf, Goebel, \& Sack, 2012; Jacobs, Goebel, \& Sack, 2012), or TMS pulse intensities (de Graaf, Cornelsen, Jacobs, \& Sack, 2011), whether TMS differentially affected subjective reports or objective performance. We have generally found a strong correspondence between TMS effects on both the objective and subjective measures, suggesting that TMS pulses affect visual processing holistically. We hypothesized that perhaps objective-subjective disruption dissociations would only become apparent at certain TMS intensities, for example if conscious-related processes in EVC are more susceptible to TMS disruption than unconscious-related processes. Yet, for TMS at -25 $\mathrm{ms}$ and $+90 \mathrm{~ms}$ (de Graaf, Cornelsen, Jacobs, \& Sack, 2011) as well as $-50 \mathrm{~ms},-20 \mathrm{~ms}$, and $+120 \mathrm{~ms}$ (previous Chapter), effects were nearly identical for both measures of vision across a wide range of intensities..

But the fact that vision as a whole is affected by TMS does not yet preclude the possibility that unconscious processing remains when conscious perception is wholly abolished. To investigate this issue, behavioral performance in trials without conscious perception must be evaluated as in Ro et al. (2004) and Boyer et al. (2005). In one recent report, we found significant above-chance performance in unconscious trials for some SOAs $(-40 \mathrm{~ms},+80 \mathrm{~ms},+100 \mathrm{~ms})$ but not in others, suggesting that perhaps the 'TMS-induced blindsight' effect may depend on the timing of TMS pulses (Jacobs, Goebel, \& Sack, 2012).

Koivisto et al. (2010) examined the issue for motion stimuli. Replicating the core findings from Silvanto et al. (2005), they stimulated early visual cortex in the 'early' and 'late' time windows, with hMT in-between, finding again that early visual cortex was functionally relevant in both time windows. But while Silvanto (2005) implemented only a subjective 'conscious' measure, now an objective 'unconscious' 2AFC discrimination task was included. Interestingly, performance on trials in which visual awareness of motion direction was absent was above-chance for no-TMS trials, for an 'early' masking window (i.e. $20 \mathrm{~ms}$ stimulus offset asynchrony), but was at chance-level for a 'late' masking window (i.e. $60 \mathrm{~ms}$ stimulus offset asynchrony), suggesting that in this paradigm the feedback was required for both conscious and 'unconscious' processing of motion. 
Sack et al. (2009) presented subjects with symbolic arrow primes (pointing to the left or to the right), which were meta-contrast masked. Even when thus not consciously perceived, the arrow primes generally improve performance when subjects discriminate the pointing-direction of the target arrows (Vorberg, Mattler, Heinecke, Schmidt, \& Schwarzbach, 2003). If TMS in the classical masking window affects only conscious processing, this symbolic response priming effect should remain unaffected by TMS. Yet, TMS at SOAs from 40-100 ms abolished response priming, as well as conscious perception of the stimuli. However, Koivisto et al. (2012) in a similar paradigm could show that TMS over EVC reduced unconscious priming at SOAs from 30 to $90 \mathrm{~ms}$, whereas conscious recognition of (unmasked) primes was impaired at SOAs from 60 to $120 \mathrm{~ms}$. Importantly, TMS over lateral occipital cortex (LO) impaired unconscious priming at SOAs from 90 to $120 \mathrm{~ms}$ (although only when the task was fresh), suggesting that unconscious priming relied on a linear feedforward sweep from V1 to higher areas. These results suggest that the end part of the classical dip (around $120 \mathrm{~ms}$ ) was, at least in this particular study, related specifically to conscious perception, and that the late part may have reflected feedback processing from higher areas because LO was functionally relevant well before the classical dip had ended.

Railo et al. (2012) studied processing of colors and found no consciousnessspecific suppression of chromatic stimuli by occipital TMS in TMS windows up to 100 ms. Conscious color detection, forced-choice color discrimination, and unconscious color priming were all abolished by TMS over EVC. Lastly, Koivisto et al. (2011) tested the effects of occipital TMS on subjective (conscious) and objective (forcedchoice) processing of two types of stimuli, symbolic arrow stimuli and orientation stimuli. TMS affected both conscious and unconscious processing of symbolic stimuli in the same classical time windows (from 60-120 ms). But for the orientation stimuli, TMS specifically suppressed conscious processing from $90-120 \mathrm{~ms}$, thus again at the 'later part' of the classical masking window. In a different paradigm, we also noted that occipital TMS in late classical SOAs (110-130 ms) affected the processing of one type of visual stimulus (faces) but not orientation stimuli (gratings). Here, both types of stimuli were superimposed and only the task differed (experiment 2 in de Graaf, Goebel, \& Sack, 2012). An idea set forth in Koivisto et al. (2011) could similarly explain both datasets, as well as several related findings discussed above. That idea requires reinterpretation of the classical dip.

\section{Revisiting the classical masking dip}

We observed several times above that the classical dip, spanning as broad a period as 60-140 ms SOAs, may not reflect a single stage of processing. One idea put forth by Koivisto and colleagues (2011 and afterward) is that the classical masking dip more or less contains two stages. The first part of the masking curve (i.e. from $\sim 70 \mathrm{~ms}$ to $\sim 100$ $\mathrm{ms}$ ) represents a feedforward stage of activity, in which early processing stages relevant for both conscious and unconscious vision, and presumably relatively independent of stimuli, tasks, context take place. But soon after this, local recurrent activity within EVC and between EVC and extrastriate areas commences, in the second part of the 
classical masking dip (i.e. $\sim 100 \mathrm{~ms}$ to $\sim 130 \mathrm{~ms}$ ), potentially determining the contents of visual awareness (Koivisto, Railo, \& Salminen-Vaparanta, 2011) and potentially susceptible to attentional/task demands (de Graaf, Goebel, \& Sack, 2012). Electrophysiological recordings in animals (Lamme \& Roelfsema, 2000) and humans (Boehler, Schoenfeld, Heinze, \& Hopf, 2008; Liu, Agam, Madsen, \& Kreiman, 2009) converge with this view in showing that the feedforward sweep reaches the highest extra-striate areas within about $100 \mathrm{~ms}$ and is immediately followed by recurrent processing in V1 about 100-120 msec after the onset of visual stimulus. It is possible that these recurrent processes may be involved with 'high-resolution' vision (e.g. Bullier, 2001; Hochstein \& Ahissar, 2002). If the stimulus or task requires such highresolution information, the latter part of the classical masking dip will appear (the crucial recurrent processes are disrupted), while it is 'missing' when these recurrent processes are not necessary for a given stimulus/task (as in the results of de Graaf et al., 2012). Alternatively, or in addition, recurrent processes taking place around 100-130 ms may be required for the establishment of conscious vision but not for rudimentary task performance (as in the results of Koivisto 2011, 2012) although this may depend on the stimuli used (e.g. Koivisto 2010, 2011).

It thus appear that much can still be gained by using TMS to investigate the 'classical masking dip', using (1) different stimuli/tasks in the same subjects, (2) using concurrently different measures of vision (subjective, forced-choice behavioral, response priming), (3) maximizing the effective temporal resolution of TMS.

\section{Conclusions and future directions}

It is somewhat ironic that we currently see most promise, when it comes to further insights in the context of TMS masking, in a deeper probing of what is actually the most established TMS masking result: the classical masking dip. It is exactly because it is so established that it may be very meaningful to study and 'use' this effect more. One approach here is the use of different kinds of stimuli or different tasks on the same stimuli. If TMS over EVC has differential effects depending on a particular task or condition, this could mean that EVC is functionally relevant specifically in one or the other task. This strategy can be applied whenever the hypothesis involves engagement of early visual regions in the tasks at hand. There has for example been recent interest in the role of early visual cortex in memory (van de Ven \& Sack, 2012). But also, the classical masking dip can be probed further with new paradigmatic approaches, such as the simultaneous measurement of multiple subjective and objective measures of visual processing, the combined variation of TMS SOA and intensity, and (f)MRI based modeling of TMS effects in/on the brain. Results to date have been considered above.

TMS masking as a field is still young. The abolishment of visual perception by a magnetic pulse to the back of the head remains one of the most tangible and impressive effects in the TMS literature. This review suggests that early visual cortex plays a causal role in visual perception during the classical masking window, but also in various other stages, up to hundreds of milliseconds after stimulus onset and even before the stimulus appears. Yet, the exact nature of the functional role of early visual 
cortex in all these different time windows remains surprisingly obscure. Moreover, it is still unclear when and for which kind of stimuli and tasks all these dips appear. And even the classical masking effect around $\sim+100 \mathrm{~ms}$ itself may yet hold further secrets. There is definitely more to learn using the TMS masking paradigm. 


\section{Part II References}

Abrahamyan, A., Clifford, C. W., Arabzadeh, E., \& Harris, J. A. (2011). Improving visual sensitivity with subthreshold transcranial magnetic stimulation. The Journal of neuroscience : the official journal of the Society for Neuroscience, 31(9), 3290-3294.

Amassian, V. E., Cracco, R. Q., Maccabee, P. J., Cracco, J. B., Rudell, A., \& Eberle, L. (1989). Suppression of visual perception by magnetic coil stimulation of human occipital cortex. Electroencephalography and clinical neurophysiology, 74(6), 458-462. Amassian, V. E., Cracco, R. Q., Maccabee, P. J., Cracco, J. B., Rudell, A. P., \& Eberle, L. (1998). Transcranial magnetic stimulation in study of the visual pathway. Journal of clinical neurophysiology : official publication of the American Electroencephalographic Society, 15(4), 288-304.

Barker, A. T., Jalinous, R., \& Freeston, I. L. (1985). Non-invasive magnetic stimulation of human motor cortex. Lancet, 1(8437), 1106-1107.

Beckers, G., \& Homberg, V. (1991). Impairment of visual perception and visual short term memory scanning by transcranial magnetic stimulation of occipital cortex. Exp Brain Res, 87(2), 421-432.

Boehler, C. N., Schoenfeld, M. A., Heinze, H. J., \& Hopf, J. M. (2008). Rapid recurrent processing gates awareness in primary visual cortex. Proc Natl Acad Sci USA, 105(25), 8742-8747.

Boyer, J. L., Harrison, S., \& Ro, T. (2005). Unconscious processing of orientation and color without primary visual cortex. Proc Natl Acad Sci U S A, 102(46), 16875-16879.

Bullier, J. (2001). Integrated model of visual processing. Brain Res Brain Res Rev, 36(2-3), 96-107.

Busch, N. A., Dubois, J., \& VanRullen, R. (2009). The phase of ongoing EEG oscillations predicts visual perception. J Neurosci, 29(24), 7869-7876.

Camprodon, J. A., Zohary, E., Brodbeck, V., \& Pascual-Leone, A. (2010). Two phases of V1 activity for visual recognition of natural images. Journal of cognitive neuroscience, 22(6), 1262-1269.

Celebrini, S., Thorpe, S., Trotter, Y., \& Imbert, M. (1993). Dynamics of orientation coding in area V1 of the awake primate. Visual neuroscience, 10(5), 811-825.

Chambers, C. D., Allen, C. P., Maizey, L., \& Williams, M. A. (2012). Is delayed foveal feedback critical for extra-foveal perception? Cortex; a journal devoted to the study of the nervous system and behavior.

Corthout, E., Hallett, M., \& Cowey, A. (2002). Early visual cortical processing suggested by transcranial magnetic stimulation. Neuroreport, 13(9), 1163-1166.

Corthout, E., Hallett, M., \& Cowey, A. (2003). Interference with vision by TMS over the occipital pole: a fourth period. Neuroreport, 14(4), 651-655.

Corthout, E., Hallett, M., \& Cowey, A. (2011). TMS-induced blinking assessed with high-speed video: optical disruption of visual perception. Experimental brain research. Experimentelle Hirnforschung. Experimentation cerebrale, 210(2), 243-250.

Corthout, E., Uttl, B., Juan, C. H., Hallett, M., \& Cowey, A. (2000). Suppression of vision by transcranial magnetic stimulation: a third mechanism. Neuroreport, 11(11), 2345-2349. 
Corthout, E., Uttl, B., Walsh, V., Hallett, M., \& Cowey, A. (1999). Timing of activity in early visual cortex as revealed by transcranial magnetic stimulation. Neuroreport, 10(12), 2631-2634.

Corthout, E., Uttl, B., Ziemann, U., Cowey, A., \& Hallett, M. (1999). Two periods of processing in the (circum)striate visual cortex as revealed by transcranial magnetic stimulation. Neuropsychologia, 37(2), 137-145.

Cousineau, D. (2005) Confidence intervals in within-subject designs: A simpler solution to Loftus and Masson's method, Tutorials in Quantitative Methods for Psychology, 1, 75-78

Cowey, A. (1964). Projection of the Retina on to Striate and Prestriate Cortex in the Squirrel Monkey, Saimiri Sciureus. Journal of neurophysiology, 27, 366-393.

Cowey, A. (2008). TMS and Visual Awareness. In E. M. Wassermann, C. M. Epstein, U. Ziemann, V. Walsh, T. Paus \& S. H. Lisanby (Eds.), The Oxford Handbook of Transcranial Magnetic Stimulation (pp. 411-430). New York: Oxford University Press.

de Graaf, T. A., Cornelsen, S., Jacobs, C., \& Sack, A. T. (2011). TMS effects on subjective and objective measures of vision: stimulation intensity and pre- versus poststimulus masking. Consciousness and cognition, 20(4), 1244-1255.

de Graaf, T. A., Goebel, R., \& Sack, A. T. (2012). Feedforward and quick recurrent processes in early visual cortex revealed by TMS? NeuroImage, 61(3), 651-659.

de Graaf, T. A., Herring, J., \& Sack, A. T. (2011). A chronometric exploration of highresolution 'sensitive TMS masking' effects on subjective and objective measures of vision. Experimental brain research. Experimentelle Hirnforschung. Experimentation cerebrale, 209(1), 19-27.

de Graaf, T. A., \& Sack, A. T. (2011). Null results in TMS: from absence of evidence to evidence of absence. Neuroscience and biobehavioral reviews, 35(3), 871-877.

DeYoe, E. A., Felleman, D. J., Van Essen, D. C., \& McClendon, E. (1994). Multiple processing streams in occipitotemporal visual cortex. Nature, 371(6493), 151-154.

Di Lollo, V., Enns, J. T., \& Rensink, R. A. (2000). Competition for consciousness among visual events: the psychophysics of reentrant visual processes. J Exp Psychol Gen, 129(4), 481-507.

Di Russo, F., Martinez, A., Sereno, M. I., Pitzalis, S., \& Hillyard, S. A. (2002). Cortical sources of the early components of the visual evoked potential. Hum Brain Mapp, 15(2), 95-111.

Dugue, L., Marque, P., \& VanRullen, R. (2011). Transcranial magnetic stimulation reveals attentional feedback to area V1 during serial visual search. PloS one, 6(5), e19712.

Felleman, D. J., Burkhalter, A., \& Van Essen, D. C. (1997). Cortical connections of areas V3 and VP of macaque monkey extrastriate visual cortex. J Comp Neurol, 379(1), 21-47.

Foxe, J. J., \& Simpson, G. V. (2002). Flow of activation from V1 to frontal cortex in humans. A framework for defining "early" visual processing. Exp Brain Res, 142(1), 139-150.

Heinen, K., Jolij, J., \& Lamme, V. A. (2005). Figure-ground segregation requires two distinct periods of activity in V1: a transcranial magnetic stimulation study. Neuroreport, 16(13), 1483-1487. 
Hochstein, S., \& Ahissar, M. (2002). View from the top: hierarchies and reverse hierarchies in the visual system. Neuron, 36(5), 791-804.

Jacobs, C., De Graaf, T. A., Goebel, R., \& Sack, A. T. (2012). The Temporal Dynamics of Early Visual Cortex Involvement in Behavioral Priming. PLoS ONE.

Jacobs, C., Goebel, R., \& Sack, A. T. (2012). Visual awareness suppression by prestimulus brain stimulation; a neural effect. NeuroImage, 59(1), 616-624.

Juan, C. H., \& Walsh, V. (2003). Feedback to V1: a reverse hierarchy in vision. Experimental brain research. Experimentelle Hirnforschung. Experimentation cerebrale, 150(2), 259-263.

Kammer, T. (2007). Masking visual stimuli by transcranial magnetic stimulation. Psychological research, 71(6), 659-666.

Kammer, T. (2008). Visual masking by transcranial magnetic stimulation in the first 80 milliseconds. Advances in cognitive psychology / University of Finance and Management in Warsaw, 3(1-2), 177-179.

Kammer, T., Puls, K., Erb, M., \& Grodd, W. (2005). Transcranial magnetic stimulation in the visual system. II. Characterization of induced phosphenes and scotomas. Experimental brain research. Experimentelle Hirnforschung. Experimentation cerebrale, 160(1), 129-140.

Kammer, T., Puls, K., Strasburger, H., Hill, N. J., \& Wichmann, F. A. (2005). Transcranial magnetic stimulation in the visual system. I. The psychophysics of visual suppression. Experimental brain research. Experimentelle Hirnforschung. Experimentation cerebrale, 160(1), 118-128.

Kammer, T., Scharnowski, F., \& Herzog, M. H. (2003). Combining backward masking and transcranial magnetic stimulation in human observers. Neuroscience letters, 343(3), 171-174.

Kammer, T., Vorwerg, M., \& Herrnberger, B. (2007). Anisotropy in the visual cortex investigated by neuronavigated transcranial magnetic stimulation. NeuroImage, 36(2), 313-321.

Knierim, J. J., \& van Essen, D. C. (1992). Neuronal responses to static texture patterns in area V1 of the alert macaque monkey. Journal of neurophysiology, 67(4), 961-980.

Koivisto, M., Henriksson, L., Revonsuo, A., \& Railo, H. (2012). Unconscious response priming by shape depends on geniculostriate visual projection. The European journal of neuroscience, 35(4), 623-633.

Koivisto, M., Mantyla, T., \& Silvanto, J. (2010). The role of early visual cortex (V1/V2) in conscious and unconscious visual perception. NeuroImage, 51(2), 828-834.

Koivisto, M., Railo, H., Revonsuo, A., Vanni, S., \& Salminen-Vaparanta, N. (2011). Recurrent processing in $\mathrm{V} 1 / \mathrm{V} 2$ contributes to categorization of natural scenes. The Journal of neuroscience : the official journal of the Society for Neuroscience, 31(7), 2488-2492.

Koivisto, M., Railo, H., \& Salminen-Vaparanta, N. (2011). Transcranial magnetic stimulation of early visual cortex interferes with subjective visual awareness and objective forced-choice performance. Consciousness and cognition, 20(2), 288-298.

Koivisto, M., \& Silvanto, J. (2012). Visual feature binding: the critical time windows of V1/V2 and parietal activity. NeuroImage, 59(2), 1608-1614. 
Lamme, V. A. (2001). Blindsight: the role of feedforward and feedback corticocortical connections. Acta psychologica, 107(1-3), 209-228.

Lamme, V. A., \& Roelfsema, P. R. (2000). The distinct modes of vision offered by feedforward and recurrent processing. Trends in neurosciences, 23(11), 571-579.

Lamme, V. A., Super, H., Landman, R., Roelfsema, P. R., \& Spekreijse, H. (2000). The role of primary visual cortex (V1) in visual awareness. Vision research, 40(10-12), 1507-1521.

Lamme, V. A., Super, H., \& Spekreijse, H. (1998). Feedforward, horizontal, and feedback processing in the visual cortex. Current opinion in neurobiology, 8(4), 529535 .

Laycock, R., Crewther, D. P., Fitzgerald, P. B., \& Crewther, S. G. (2007). Evidence for fast signals and later processing in human V1/V2 and V5/MT+: A TMS study of motion perception. J Neurophysiol, 98(3), 1253-1262.

Liu, H., Agam, Y., Madsen, J. R., \& Kreiman, G. (2009). Timing, timing, timing: fast decoding of object information from intracranial field potentials in human visual cortex. Neuron, 62(2), 281-290.

Mathewson, K. E., Gratton, G., Fabiani, M., Beck, D. M., \& Ro, T. (2009). To see or not to see: prestimulus alpha phase predicts visual awareness. J Neurosci, 29(9), 27252732.

Moliadze, V., Zhao, Y., Eysel, U., \& Funke, K. (2003). Effect of transcranial magnetic stimulation on single-unit activity in the cat primary visual cortex. The Journal of physiology, 553(Pt 2), 665-679.

Nowak, L. G., Munk, M. H., Girard, P., \& Bullier, J. (1995). Visual latencies in areas V1 and V2 of the macaque monkey. Vis Neurosci, 12(2), 371-384.

Pascual-Leone, A., \& Walsh, V. (2001). Fast backprojections from the motion to the primary visual area necessary for visual awareness. Science, 292(5516), 510-512.

Pascual-Leone, A., Walsh, V., \& Rothwell, J. (2000). Transcranial magnetic stimulation in cognitive neuroscience--virtual lesion, chronometry, and functional connectivity. Curr Opin Neurobiol, 10(2), 232-237.

Paulus, W., Korinth, S., Wischer, S., \& Tergau, F. (1999). Differential inhibition of chromatic and achromatic perception by transcranial magnetic stimulation of the human visual cortex. Neuroreport, 10(6), 1245-1248.

Pollen, D. A. (2003). Explicit neural representations, recursive neural networks and conscious visual perception. Cerebral cortex, 13(8), 807-814.

Railo, H., \& Koivisto, M. (2012). Two means of suppressing visual awareness: a direct comparison of visual masking and transcranial magnetic stimulation. Cortex; a journal devoted to the study of the nervous system and behavior, 48(3), 333-343.

Railo, H., Salminen-Vaparanta, N., Henriksson, L., Revonsuo, A., \& Koivisto, M. (2012). Unconscious and conscious processing of color rely on activity in early visual cortex: a TMS study. Journal of cognitive neuroscience, 24(4), 819-829.

Ro, T., Shelton, D., Lee, O. L., \& Chang, E. (2004). Extrageniculate mediation of unconscious vision in transcranial magnetic stimulation-induced blindsight. Proceedings of the National Academy of Sciences of the United States of America, 101(26), 9933-9935. 
Sack, A. T., van der Mark, S., Schuhmann, T., Schwarzbach, J., \& Goebel, R. (2009). Symbolic action priming relies on intact neural transmission along the retino-geniculostriate pathway. Neuroimage, 44(1), 284-293.

Salminen-Vaparanta, N., Koivisto, M., Noreika, V., Vanni, S., \& Revonsuo, A. (2012). Neuronavigated transcranial magnetic stimulation suggests that area V2 is necessary for visual awareness. Neuropsychologia, 50(7), 1621-1627.

Salminen-Vaparanta, N., Noreika, V., Revonsuo, A., Koivisto, M., \& Vanni, S. (2012). Is selective primary visual cortex stimulation achievable with TMS? Human brain mapping, 33(3), 652-665.

Silvanto, J., Cowey, A., Lavie, N., \& Walsh, V. (2005). Striate cortex (V1) activity gates awareness of motion. Nat Neurosci, 8(2), 143-144.

Silvanto, J., Lavie, N., \& Walsh, V. (2005). Double dissociation of V1 and V5/MT activity in visual awareness. Cereb Cortex, 15(11), 1736-1741.

Stevens, L. K., McGraw, P. V., Ledgeway, T., \& Schluppeck, D. (2009). Temporal characteristics of global motion processing revealed by transcranial magnetic stimulation. Eur J Neurosci, 30(12), 2415-2426.

Thielscher, A., Reichenbach, A., Ugurbil, K., \& Uludag, K. (2010). The cortical site of visual suppression by transcranial magnetic stimulation. Cerebral cortex, 20(2), 328338.

Thut, G., Nietzel, A., Brandt, S. A., \& Pascual-Leone, A. (2006). Alpha-band electroencephalographic activity over occipital cortex indexes visuospatial attention bias and predicts visual target detection. J Neurosci, 26(37), 9494-9502.

van de Ven, V., \& Sack, A. T. (2012). Transcranial magnetic stimulation of visual cortex in memory: Cortical state, interference and reactivation of visual content in memory. Behav Brain Res, 236C, 67-77.

Vanni, S., Warnking, J., Dojat, M., Delon-Martin, C., Bullier, J., \& Segebarth, C. (2004). Sequence of pattern onset responses in the human visual areas: an fMRI constrained VEP source analysis. Neuroimage, 21(3), 801-817.

Vorberg, D., Mattler, U., Heinecke, A., Schmidt, T., \& Schwarzbach, J. (2003). Different time courses for visual perception and action priming. Proc Natl Acad Sci US A, 100(10), 6275-6280.

Wassermann, E. M., Epstein, C. M., Ziemann, U., Walsh, V., Paus, T., \& Lisanby, S. H. (2008). The Oxford Handbook of Transcranial Stimulation. New York: Oxford University Press.

Weiskrantz, L. (1996). Blindsight revisited. Curr Opin Neurobiol, 6(2), 215-220.

Wilson, C. L., Babb, T. L., Halgren, E., \& Crandall, P. H. (1983). Visual receptive fields and response properties of neurons in human temporal lobe and visual pathways. Brain : a journal of neurology, 106 (Pt 2), 473-502. 


\section{Part III}

High-level Aspects of Vision 



\title{
III. 1
}

\section{On the Functional Relevance of Frontal}

\author{
Cortex for Passive and Voluntarily
}

Controlled Bistable Vision

Related publication(s):

De Graaf, TA, de Jong, MC*, Goebel, R, van Ee, R*, Sack, AT (2011). On the Functional Relevance of Frontal Cortex for Passive and Voluntarily Controlled Bistable Vision. Cerebral Cortex. 21: 2322-2331

* Maartje de Jong

1) Department of Physics of Man, Helmholtz Institute, Utrecht University, Utrecht 3584 $\mathrm{CH}$, The Netherlands

* Raymond van Ee

1) Department of Physics of Man, Helmholtz Institute, Utrecht University, Utrecht 3584 $\mathrm{CH}$, The Netherlands

2) Department of Brain, Body, Behaviour, Philips Research Laboratories, Eindhoven $5656 \mathrm{AE}$, The Netherlands

3) Department of Experimental Psychology, Leuven University, Leuven B-3000, Leuven, Belgium 


\begin{abstract}
In bistable vision, one constant ambiguous stimulus leads to two alternating conscious percepts. This perceptual switching occurs spontaneously but can also be influenced through voluntary control. Neuroimaging studies have reported that frontal regions are activated during spontaneous perceptual switches, leading some researchers to suggest that frontal regions causally induce perceptual switches. But the opposite also seems possible: frontal activations may themselves be caused by spontaneous switches. Classically implicated in attentional processes, these same regions are also candidates for the origins of voluntary control over bistable vision. Here too, it remains unknown whether frontal cortex is actually functionally relevant. It is even possible that spontaneous perceptual switches and voluntarily induced switches are mediated by the same top-down mechanisms. To directly address these issues, we here induced "virtual lesions," with transcranial magnetic stimulation, in frontal, parietal, and 2 lower level visual cortices using an established ambiguous structure-from-motion stimulus. We found that dorsolateral prefrontal cortex was causally relevant for voluntary control over perceptual switches. In contrast, we failed to find any evidence for an active role of frontal cortex in passive bistable vision. Thus, it seems the same pathway used for willed top-down modulation of bistable vision is not used during passive bistable viewing.
\end{abstract}




\section{Introduction}

Visual input is rarely unambiguous. The brain has evolved to resolve ambiguities, making sure that our conscious experiences constitute coherent wholes. In the laboratory, visual ambiguity can be taken to extremes in order to study the brain mechanisms underlying the establishment of coherent conscious vision. If one ambiguous stimulus is presented continuously, our experience will switch back and forth between the 2 (or more) possible percepts. This is called multistable perception (Rees et al. 2002; Kim and Blake 2005; Sterzer et al. 2009). Since it is only the conscious experience that changes, and not the stimulus, concurrent changes in brain activity must reflect the contents, consequences, or establishment (prerequisites) of visual awareness (de Graaf et al., 2012).

Often using bistable paradigms, imaging studies in humans have concluded that activity changes in extrastriate (Kleinschmidt et al. 1998; Lumer et al. 1998; Tong et al. 1998; Polonsky et al. 2000; Meng et al. 2005; Moutoussis et al. 2005; Hsieh et al. 2006; Sterzer and Rees 2008; Hsieh and Tse 2009, 2010) and striate (Polonsky et al. 2000; Tong and Engel 2001; Lee and Blake 2002; Lee et al. 2005; Meng et al. 2005; Hsieh et al. 2006; Hsieh and Tse 2010) visual cortex and even subcortical visual nuclei (Haynes et al. 2005; Wunderlich et al. 2005) correlate to changes in conscious percept, rather than changes in stimulation.

Interestingly, aside from such low-level visual regions, higher order regions have been implicated. Already in 1998, Lumer et al. reported in a pioneering functional magnetic resonance imaging (fMRI) study that widespread frontoparietal regions are activated during bistable perception. Indeed, several studies have confirmed that frontal regions are somehow involved in perceptual switching during binocular rivalry and other bistable paradigms (e.g., Kleinschmidt et al. 1998; Lumer et al. 1998; Lumer and Rees 1999; Sterzer and Rees 2008; Sterzer et al. 2009; Zaretskaya et al. 2010). This is of additional interest since frontal cortex, and connectivity thereof to lower level visual regions, has been related to the establishment of conscious vision (Lumer and Rees 1999; Amassian et al. 2008).

An open question concerns the precise role frontal cortex plays in the resolution of ambiguity. Some postulate top-down influences: frontal regions might provide an impetus to earlier visual brain regions to reevaluate the visual input (Rees 2004; Sterzer et al. 2009). This would constitute an active role, effectively suggesting that frontal regions "drive" or "cause", the perceptual switches. Frontoparietal regions implicated in perceptual switching (Kleinschmidt et al. 1998; Lumer et al. 1998; Lumer and Rees 1999; Inui et al. 2000; Sterzer et al. 2002; Schoth et al. 2007; Zaretskaya et al. 2010) can overlap with the frontoparietal attention network (e.g., Coull et al. 1996; Corbetta 1998; Nobre et al. 1999; Pessoa et al. 2003; Naghavi and Nyberg 2005). Indeed, several researchers have suggested that perceptual reorganization or reconfiguration in the visual system may be instigated by higher order regions (e.g., Leopold and Logothetis 1999; Rees 2004; Slotnick and Yantis 2005; Pitts, Nerger, and Davis 2007; Pitts, Gavin, and Nerger 2008; Sterzer et al. 2009). This suggests that a form of (selective) attention may be responsible for perceptual switching in bistable vision. 
But exactly because frontal cortex has traditionally also been implicated in attention, (endogenous) perceptual switches might be salient bottom-up attentiongrabbers, causing the frontal activity (i.e. as a neural consequence) rather than the other way around. So, a resemblance between frontal activations for bistable vision and for attention does not necessarily imply that the frontal activations actually cause the perceptual switches. And indeed, alternative explanations for widespread frontoparietal activation changes have been provided (Kamphuisen et al. 2008; Raemaekers et al. 2009). Thus, it remains an open question: is frontal cortex functionally relevant for bistable vision or not?

One path to the resolution of this debate may involve the concurrent investigation of a related issue. Here we studied not only passive bistable vision but also voluntarily controlled bistable vision. It has repeatedly been shown that, under certain circumstances, people are able to control their bistable perception, inducing more frequent or less frequent switches between the competing conscious percepts (Pelton and Solley 1968; Liebert and Burk 1985; Horlitz and O'Leary 1993; Hol et al. 2003; Toppino 2003; Meng and Tong 2004; van Ee et al. 2005; Brouwer and van Ee 2006; Windmann et al. 2006; Kornmeier et al. 2009). Particularly in light of aforementioned top-down, attentional hypotheses of ambiguity resolution in the visual system, the potential insights to be gleaned from simultaneous study of intentional and nonintentional perceptual switches have recently been recognized (Slotnick and Yantis 2005; Windmann et al. 2006; Pitts, Gavin, and Nerger 2008; Kornmeier et al. 2009). It seems that attention-based theories of bistable vision might predict that the same topdown pathway, involved in voluntarily induced perceptual switching, might be involved in spontaneous switching. Yet, the neural origins of both passive and voluntarily controlled perceptual switches remain unclear, particularly concerning the role of higher order top-down regions.

In the current project, we therefore attempted to elucidate the role of frontal cortex in both passive and voluntarily controlled bistable vision. We used an ambiguous bistable structure-from-motion (SFM) stimulus (Fig. 1A) that has previously been shown amenable to voluntary control without being confounded by eye movements or covert dot tracking (Brouwer and van Ee 2006). Perhaps the most direct and valid way to investigate whether certain brain regions are functionally relevant for a given task is to transiently interfere with brain activity in those regions and subsequently evaluate potential effects on task performance. In the current study, if frontal regions are causing or "driving" the perceptual switches during passive bistable vision, as has been proposed (see above), a "virtual lesion" of these regions should alter the rate of switching. Similarly, if these regions are the source of voluntary control over bistable vision, "virtual lesions" of these regions should reduce the ability to exercise this control. To induce such virtual lesions, we administered offline inhibitory repetitive transcranial magnetic stimulation (rTMS) over 2 high-level regions (frontal and parietal cortices) and 2 low-level regions (occipital pole and the human motion area: hMT/ V5-see Materials and Methods) of the visual system, in separate sessions but in the same subjects, to evaluate potential effects on spontaneous switch rate during passive viewing and on voluntary control over switch rate during controlled viewing. 
A

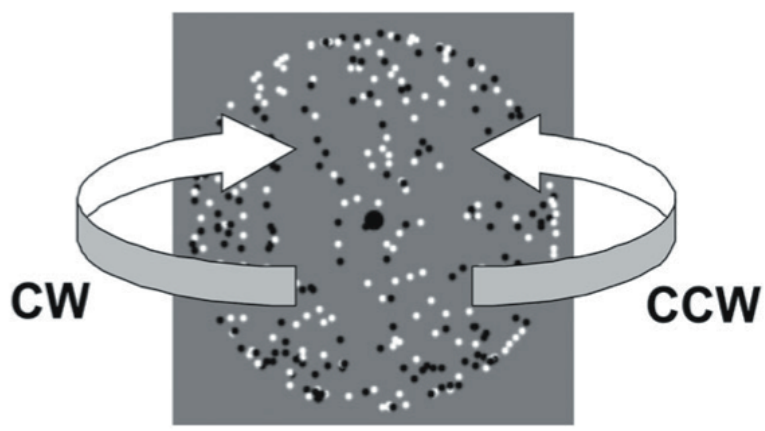

B
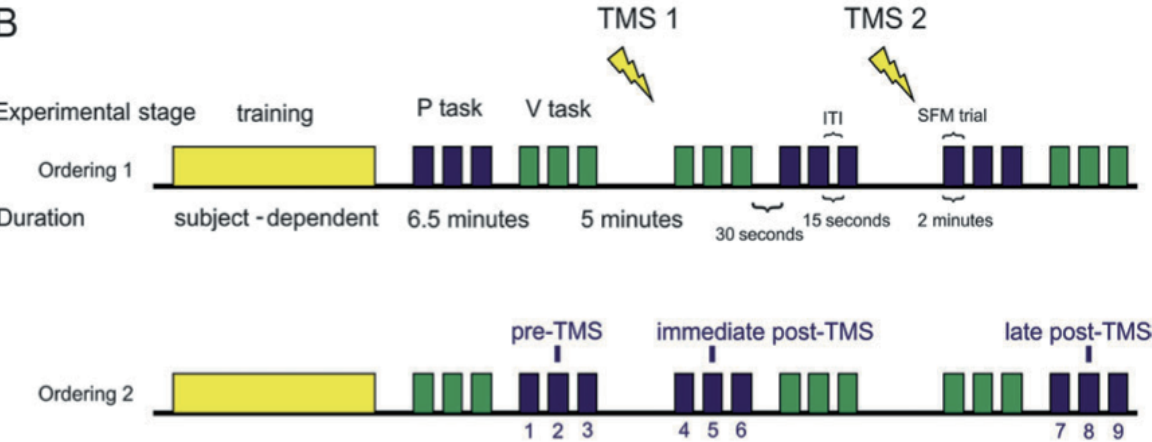

Figure 1. Stimulus, tasks, design.

(A) One of 190 bitmaps constituting the structure-from-motion (SFM) stimulus is shown. During the experiment, 190 bitmaps with slightly different dot positions were presented in rapid succession, resulting in a fluidly rotating sphere perception. The direction of rotation was ambiguous; perceived direction was indicated with button presses. (B) Shown are the 2 possible orderings of task blocks. One experimental order (shown in the top depiction) consisted of P (passive), V (voluntary control) - rTMS (5 min of $1 \mathrm{~Hz}$ TMS) - V, PrTMS - P, V. Effectively, this meant that in this session both V and P tasks were executed once immediately after TMS, once pre- TMS, and once $7 \mathrm{~min}$ after TMS. The alternative order is presented below. These 2 orderings were counterbalanced within and between subjects and sites. Note that the data collected in these sessions could be reconfigured post hoc to obtain one timeline per task related to one TMS administration. This reconfigured timeline underlies Figures 3 and 4B and Supplementary Figure S1. ITI, intertrial interval.

\section{Materials and Methods}

\section{Participants}

Fourteen subjects participated in this study. Three subjects did not complete all 4 sessions because they failed to control their perception. One subject was excluded 
because of an exceedingly high motor threshold (resulting in disproportionate experimental TMS intensity). The 10 remaining subjects (5 males, age range 21--26 years) all had normal or corrected-to-normal vision and no history of neuropsychiatric disorders. The experiment was approved by the local medical-ethical committee, and written informed consent was obtained before participation. Participants were screened for TMS experimentation safety by a medical supervisor and received monetary compensation.

\section{Stimuli and Tasks}

Stimuli were presented on a standard TFT computer monitor, using Presentation software (Neurobehavioral Systems). Viewing distance was $60 \mathrm{~cm}$. The ambiguous sphere (SFM), rotating around the vertical axis (width/height: $4.8^{\circ}$, density: 300 dots; see Fig. 1), was created using custom software. The dots that constituted the sphere measured 6.2 arcmin in width and height. Half of the dots were white, the other half black, presented on a uniform gray background, so that overall luminance was equal and no luminance adaptation should occur. The SFM contained a central fixation dot of 13.8 arcmin; angular velocity of the sphere was $57.1 \%$ s. The dots moved back and forth horizontally. Their speed profile mimicked that of a flat projection of dots scattered on the surface of a transparent globe revolving around its central vertical axis (i.e., a sinusoidal speed profile). This type of display readily elicits the illusion of the full, 3D, structure (e.g., Wallach and O'Connel 1953). Due to a lack of additional depth cues indicating which motion direction corresponded to the front and which to the back surface of the sphere, observers alternately perceived either rotation direction (Fig. 1A) (e.g., Braunstein 1977). Less commonly, the same stimulus may in some cases be perceived as 2 "half-spheres" that both point outward toward the observer while sliding in opposite directions, one behind the other (Hol et al. 2003; Chen and He 2004). Although our observers did not spontaneously report this perception, we preempted any confusion it might cause by instructing observers to report the motion direction of the surface perceived to be in front, regardless of whether the hind surface was convex or concave. Note that our stimulus was identical to those employed previously (Brouwer and van Ee 2006; Brascamp et al. 2010).

Subjects either engaged in passive viewing ( $\mathrm{P}$ : only report perceived motion direction) or voluntary control (V: switch perceived motion direction as frequently as possible) tasks. At all times, subjects were explicitly instructed to refrain from using any form of motor or eye movement activity to influence their perception. Previous studies have found no consistent relationship between eye movements and perception of the ambiguously rotating sphere (Brouwer and van Ee 2006, 2007; Klink et al. 2008; see Supplementary Material for an elaboration on potential eye movement effects in our data). Rather, participants were told to use only their "mind force" to induce the opposite direction of motion. All 10 included participants reported able and confident in this task. Thus, the tasks were identical to those in Brouwer and van Ee (2006), who showed that under these conditions voluntary control over SFM is possible even after controlling for eye movements and covert tracking of the moving dots. 


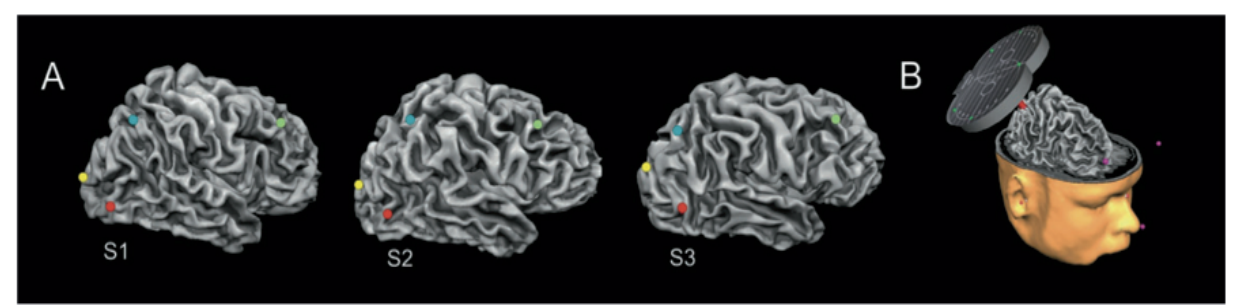

Figure 2. TMS targeted sites.

(A) Shown for 3 representative subjects are their individual reconstructed brain anatomies with TMS targeted sites superimposed in color-coded dots. (B) Illustration of neuronavigation as used and visualized in Brainvoyager.

\section{Procedure and Design}

Participants were familiarized with the tasks in a training phase, which lasted until subjects felt able to induce switches without reverting to eye or motor movements. On average, this took 6 SFM trials of V task. One trial consisted of $2 \mathrm{~min}$ of continuous SFM stimulation. Throughout the experiment, task blocks consisted of clusters of 3 SFM trials, with $15 \mathrm{~s}$ in-between trials. The experimental session (after training) included 3 parts: a pre-TMS baseline section, a first post-TMS section (immediate, iPost-TMS), and a second post-TMS section (late, 1-Post- TMS). Each of these 3 sections consisted of 2 task blocks: 1 passive (P) cluster (with 3 SFM trials) and 1 voluntary control (V) cluster (with 3 SFM trials). The order of P and V clusters was counterbalanced within and between subjects and across stimulation sites. There were 2 possible (counterbalanced) orderings of task blocks such that $1 \mathrm{P}$ cluster immediately followed TMS and $1 \mathrm{~V}$ cluster immediately followed TMS (see Fig. 1B). A break of 30 $\mathrm{S}$ was scheduled in between the 2 tasks in each section of the experimental session. Note that the measurements in this design can be reconfigured post hoc into a timeline for both the $\mathrm{P}$ and the $\mathrm{V}$ task in reference to a single offline TMS period (as exemplified in Fig. 1B for $\mathrm{P}$ and applied in Figs 3 and 4). In breaks and during TMS, lights in the lab were fully on to prevent and reverse possible dark adaptation and fatigue. During task performance, lights were dimmed.

\section{TMS Parameters}

TMS was administered in line with safety guidelines in Rossi et al. (2009). TMS involved offline rTMS for $5 \mathrm{~min}$ at $1 \mathrm{~Hz}$, resulting in 300 pulses per stimulation, twice per session. Biphasic pulses were administered with a figure-8 coil (MC-B70), over parietal cortex, frontal cortex, occipital pole, and hMT/V5, on separate days over the course of several weeks. Stimulation intensity consisted of $110 \%$ of individual motor threshold (newly determined prior to TMS in each session). However, to account for differences in coil-cortex distance, stimulation intensity at cortex level over hMT/V5 was kept constant in relation to actual stimulation intensity at cortex level over occipital cortex, in individual subjects based on their individual brain anatomies, by correcting with $3 \%$ intensity per millimeter deviation (Stokes, Chambers, Gould, Henderson, et al. 
2005; Stokes, Chambers, Gould, English, et al. 2007). Correction was limited to between $110 \%$ MT and $125 \%$ MT, to stay within safety guidelines. The right hemisphere was stimulated since literature consistently indicated right-hemispheric activations in bistable paradigms (Kleinschmidt et al. 1998; Lumer et al. 1998; Lumer and Rees 1999; Sterzer et al. 2002; Brouwer and van Ee 2007; Raemaekers et al. 2009). Frontal and parietal cortex localization was guided by the international 10/20 electroencephalography (EEG) system, P4 and F4 indicating parietal and frontal cortices, respectively, and evaluated using stereotactic frameless neuronavigation to individual brain anatomy obtained with MRI scans (Fig. 2). Resulting coordinates of stimulated sites are presented in the Results section. Occipital cortex localization was guided by anatomical landmark ( $2 \mathrm{~cm}$ above the inion) but was ensured to reflect the occipital pole, evaluated using online neuronavigation. hMT/V5 targeting was based on known Talairach coordinates but adapted on the basis of individual brain anatomy under guidance of a probabilistic map of hMT/V5 obtained in 15 independent subjects (provided by M. A. Frost; Frost and Goebel 2009). Talairach coordinates were spatially transformed to individual anatomical space to guide online localization. Actually stimulated sites in individual anatomical space were transformed inversely to Talairach space, and the resulting coordinates for each stimulated site in each subject are listed in Supplementary Table S3 and demonstrated for 3 participants in Figure 2A.

During stimulation, the coil handle pointed lateral-posterior (45 degree angle to the midline) for parietal cortex, pointed medial-posterior (45 degree angle to the midline) for frontal cortex, pointed lateral for occipital cortex, and pointed anterior for hMT/V5. Initial current direction of the biphasic pulse always flowed away from the coil handle.

\section{Analysis}

For each SFM trial, an average percept duration (PD) was calculated (a measure inversely related to the perceptual switch count). There was substantial variation in PDs between subjects and in PDs within-subject between-blocks over the course of the experiment (due to practice, fatigue, motivation, arousal/alertness immediately after rTMS, and so on). Since SFM trials occurred in clusters of 3 per block, we normalized per subject, per task block, the PD in the first and the second SFM trial to the PD in the third SFM trial. This procedure resulted in normalized average percept durations (nPDs). Outliers were removed (see Supplementary Material).

TMS effects were only expected in the first SFM trial immediately after TMS since we used cognitive tasks and applied 1-Hz rTMS for only 5 min (Wassermann et al. 2008). We applied a repeated measures analysis of variance (ANOVA) on these trials with factors TMS site (4 levels) and task (2 levels) to establish initial main effects and interactions between TMS site and task. Subsequently, we evaluated per task and site whether TMS had an influence: we applied (uncorrected) one-tailed t-tests on the time windows of interest (first SFM trial immediately after rTMS: SFM4) for all 4 TMS target sites. However, to make sure our results were not an artifact of the applied normalization procedure, we analyzed the data with an additional normalization procedure that involved only one step of normalization rather than normalization within 
each task block. This is presented in the Supplementary Material and exactly reproduces the pattern of effects presented here.

In a second, fundamentally different form of analysis, we evaluated the TMS effects on the distributions of PDs rather than the mean PD. We fit a gamma distribution to the PDs, per SFM, and extracted the scale and shape parameters of this distribution. Here, we first divided per participant each PD value by the mean PD of that participant, before fitting all PDs to a gamma distribution per time window, site, and task. For the formula of the used gamma distribution, we refer to Brouwer and van Ee (2006), where the same fit procedure was used. To statistically compare the gamma fits of different time windows, we used t-tests based on gamma parameters and confidence intervals thereof, using Matlab software (Mathworks). For these analyses, we corrected for the large number of comparisons using a Bonferroni correction. TMS over frontal cortex affected voluntary control even with this overly strict correction and did not affect passive viewing even without this correction $(a=0.05)$. See below for all results. Thus, importantly, all analyses and normalization procedures support the same frontal results.

\section{Results}

Our participants engaged in several blocks of 3 consecutive trials ( 2 min each), in which they either passively viewed ( $\mathrm{P}$ task) the SFM stimulus and reported its perceived rotation direction or voluntarily tried to make the perceived direction switch as often as possible (V task) while reporting the perceived rotation direction. The results are shown in Figure 3 for all these 2-min trials. Measurements for each task were obtained 3 times per session: before TMS, immediately after TMS, or later ( $>8 \mathrm{~min}$ ) after TMS (Fig. 1B, see Materials and Methods). With the TMS protocol applied, we expected TMS effects only in the first SFM trial in the i-Post-TMS block. Data from these trials of interest are shown alongside the other trials to provide a complete reference frame (trials of interest are highlighted orange in all Results figures: note that they reflect trials where TMS effects should occur if present, not trials where comparisons were statistically significant per se). Localization of TMS target sites was achieved using a combination of frameless stereotactic neuronavigation (Fig. 2B), individual MRI anatomy measurements guided by known Talairach coordinates, anatomical landmarks, the international EEG 10/20 system, and fMRI probabilistic mapping of functional regions (see Materials and Methods, and see Fig. 2 and Supplementary Table S3 for resulting TMS targeted sites). We can here report coordinates of the actually stimulated higher order regions of interest. Frontal and parietal cortices were initially localized using the EEG 10/20 system and post hoc identified with individual MRI-guided neuronavigation. The parietal region we stimulated was mean Tal $[\mathrm{x}, \mathrm{y}, \mathrm{z}]=[27,-71$, 42], average deviations [7, 7, 5]—-see Supplementary Table S3 for details-which corresponds to the superior parietal lobule/precuneus. The frontal gray matter closest to the average coordinates: Tal $[\mathrm{x}, \mathrm{y}, \mathrm{z}]=[25,27,43]$, average deviations $[4,8,4]-$ see Supplementary Table S3 - corresponds to the middle frontal gyrus. Since there was some variability of individual target coordinates around these means (see Fig. 2A and 
A

Parietal - passive

Parietal - voluntary
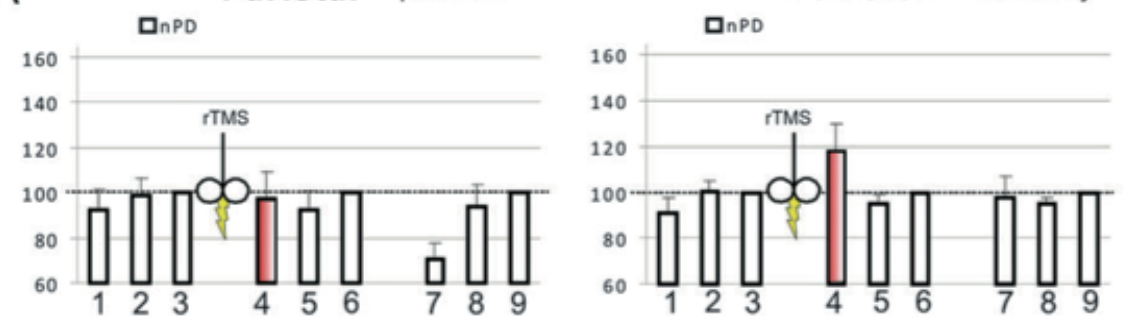

B

Frontal - passive

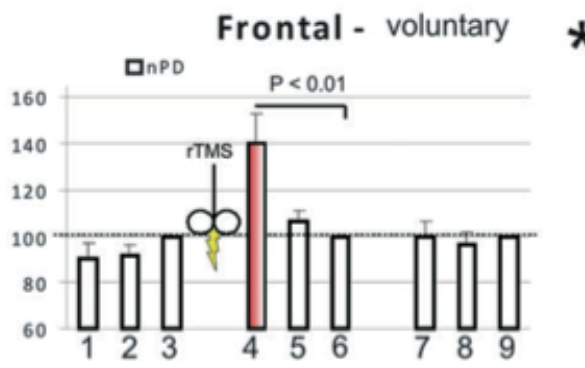

C

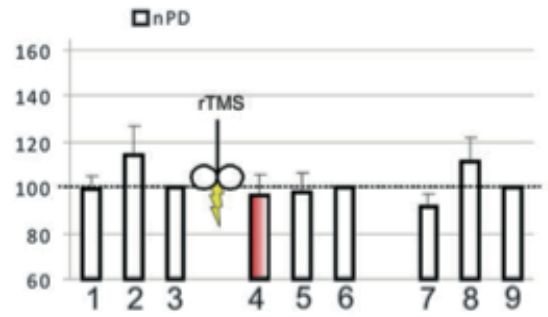

hMT - passive
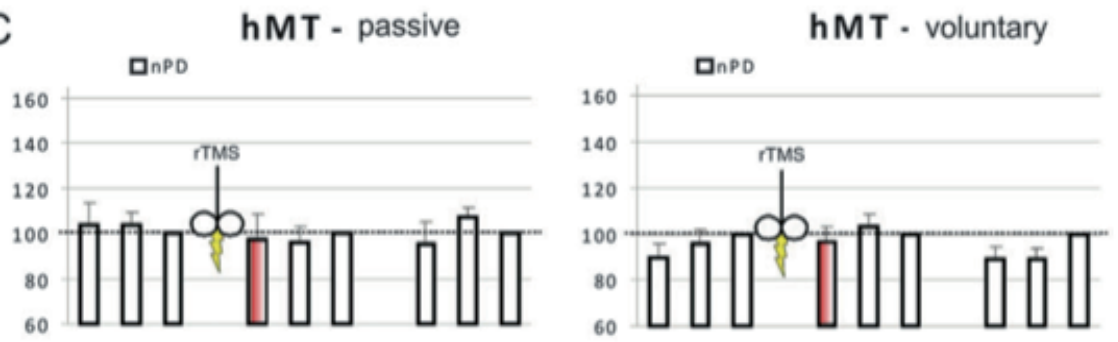

D
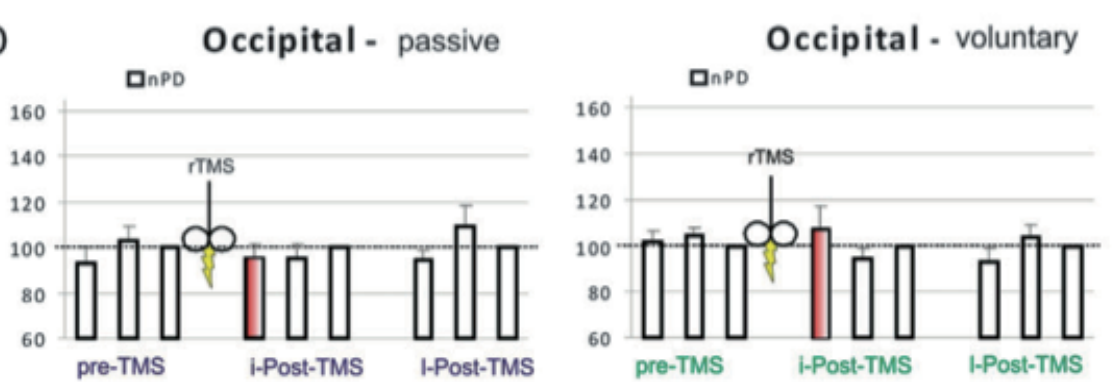

Figure 3: Behavioral and TMS results.

(A) The results are shown for passive viewing (P: left plot) and voluntary control task (V: right plot) for parietal cortex stimulation. On the horizontal axis, Arabic numerals indicate the time window of the trial relative to rTMS administration. Each time window represents one SFM trial, lasting 2 min. Time windows 1, 2, 3 are pre-TMS, 4, 5, 6 immediately after rTMS, 7, 8, 9 later after rTMS (see Fig. 1B). On the vertical axis, normalized percept durations nPDs are presented in percentages. Error bars indicate standard error of the mean. (See also Supplementary Figure S1 for alternative normalization results.) (B) Same as in (A) but for frontal cortex. The asterisk on the right panel indicates a statistically significant deviation from $100 \%$ in time window 4 . This indicates that, for the first 2 min after rTMS, voluntary control over bistable perception was significantly reduced: participants were less able to make the perceived rotation direction switch frequently (leading to relatively increased percept durations).(C) Same as in (A) but for hMT/V5 stimulation. (D) Same as im(14) but for occipital pole stimulation. 
primarily Supplementary Table S3), we conservatively conclude that we stimulated posterior parietal cortex and dorsolateral prefrontal cortex.

It has been noted before that some observers are more effective at exerting voluntary control over bistable perception (Borsellino et al. 1982; Struber and Stadler 1999; Struber et al. 2000; Pitts, Gavin, and Nerger 2008), but our sample size did not allow a rigorous examination of the issue. Importantly, we confirmed that all 10 included participants were consistently able to perform the voluntary control task (see Supplementary Material-notably Supplementary Table S1 in which all nonnormalized percept durations are shown, allowing a comparison of PDs between the $\mathrm{P}$ and $\mathrm{V}$ conditions).

We analyzed the data on 2 very different levels. We first analyzed the average PDs in different conditions (see Materials and Methods). Second, we investigated the distributions of PDs, rather than their means. For this analysis, we fitted the PDs to gamma distributions, extracting the scale and shape parameters. We then evaluated TMS effects on these distributions.

In the average PD analysis, to resolve inter- and intra-individual variations we normalized the average PDs (see, e.g., Meng and Tong 2004) to the third SFM trial per task block (see Materials and Methods). In the Supplementary Material, we present the results of an alternative one-step normalization procedure-leading to the same (statistical) interpretations and conclusions. All analyses thus supported the same pattern of results presented here.

A repeated measures group ANOVA on the trials of interest revealed a trend for interaction between task and TMS site on the nPD $(\mathrm{F}=2.36, \mathrm{P}<0.1)$, motivating us to investigate the effects of TMS per task and site (see Fig. 3).

\section{Frontal Cortex in Passive Viewing}

In our setup, if frontal regions were somehow responsible for passive switches through top-down signals, inhibitory rTMS should change nPD scores (e.g., higher nPDs: reflecting fewer switches). We will discuss below that voluntary control was significantly inhibited by frontal rTMS. However, as illustrated in Figure 3A (left), B (left), there was no evidence for any rTMS effect in either parietal or frontal regions on passive viewing. The small deviations from $100 \%$ that were revealed were convincingly nonsignificant (parietal SFM4 vs. 100\%: t9 $=-0.22, \mathrm{P}=0.83$; frontal SFM4 vs. $100 \%$ : t9 $=-0.35, P=0.74)$. This is unlikely to be attributable to either floor or ceiling effects of passive viewing switch rates since both higher and lower switch rates for passive viewing have been observed in the same participants across the many conditions of the current study (see Supplementary Table S1).

This pattern was confirmed by the gamma fit analyses. Figure 4A illustrates histograms for the trials immediately after TMS (SFM trial 4) and the first pre-TMS trials for comparison (SFM trial 1). In these histograms, no consistent changes can be seen for passive viewing (in contrast to voluntary control, see below). The gamma curves representing the distribution of PDs are illustrated in small insets, with the orange curves reflecting SFM trial 4 and blue thin curves reflecting the comparison trial. 

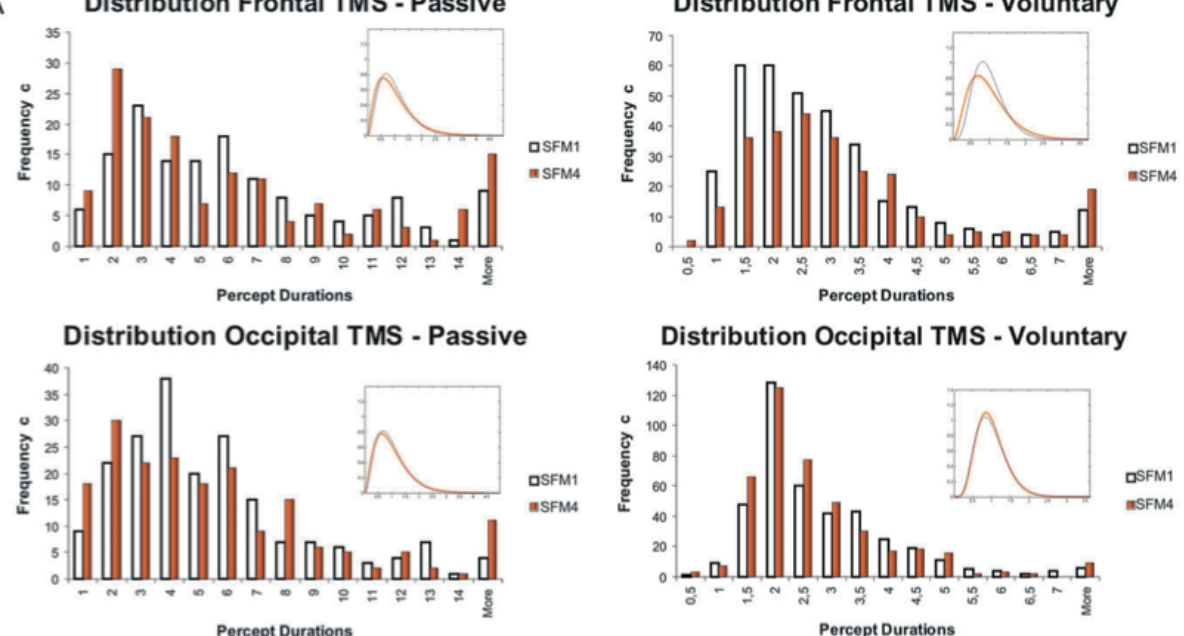

Distribution Occipital TMS - Voluntary
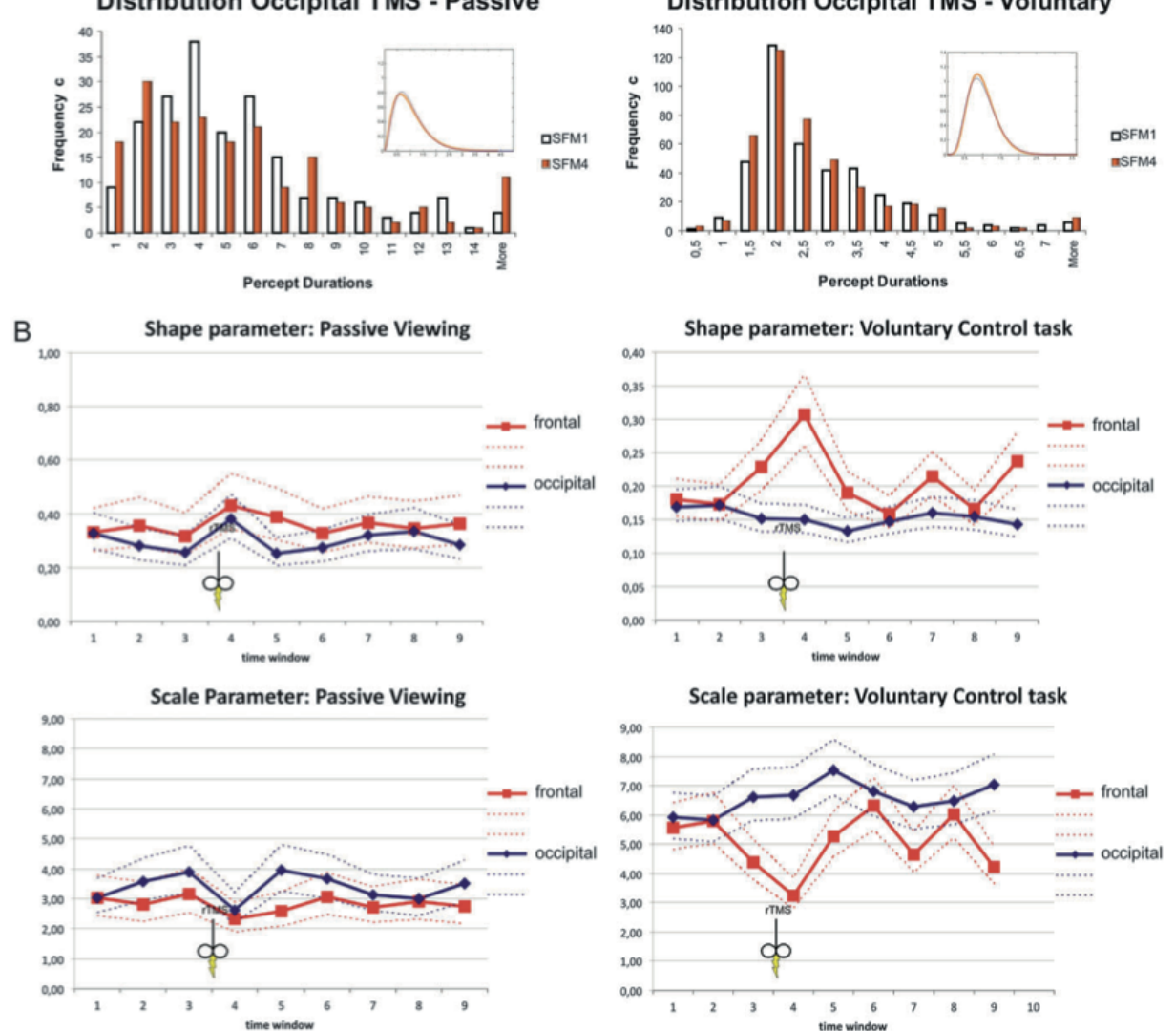

Figure 4. Gamma distributions and parameters.

(A) A histogram of raw, non-normalized percept durations (PDs) is shown of the trials of interest (immediately after rTMS; SFM4 5 SFM trial 4-see Fig. 1B, in orange) and of a control trial (SFM1 5 first pre-TMS trial) for comparison. This is illustrated for frontal cortex both voluntary control (upper right) and passive viewing (upper left) and occipital cortex for comparison. The gamma curves (see Materials and Methods) corresponding to these conditions are presented in small insets (orange again representing the trials of interest and thin blue representing the control trial). Already by eye, it is clear that TMS caused a shift in distribution of PDs, and in the corresponding gamma curve (inset), for frontal cortex and voluntary control. For passive viewing, and occipital cortex, this is not evident. (B) To illustrate graphically the pattern of gamma fit parameters over all time windows, both shape and scale parameter are plotted for frontal (red curves) and occipital (blue curves) cortices for voluntary control (right plots) and passive viewing (left plots). Dashed lines surrounding the curves represent the upper and lower bounds of $95 \%$ confidence intervals - to provide an indication of variability and thus reliability of the curves. Note that no within-block or between-block normalization took place. Nonetheless, a clear TMS effect at the trials of interest (SFM4 5 first SFM trial after TMS) is observed on both gamma fit parameters for 2 lffontal cortex and voluntary control. 
Statistically, we compared gamma fits of the trials of interest with 2 control distributions, to keep the analysis analogous to the average PD analyses. Thus, the gamma fits of the trials of interest were compared with SFM trial 6 (a within-task block comparison) and with all pre-TMS baseline trials collapsed (analogous to the betweenblock comparison underlying Supplementary Figure S1). For no TMS target site was the gamma fit of the trials of interest significantly different from these control gamma fits. We should note that, even if the comparison was not corrected for multiple comparisons, there was still no significant TMS effect on passive viewing for frontal cortex (in contrast to voluntary control, see below). All gamma shape and scale parameters describing all distributions can be found in Supplementary Table S3.

Figure 4B shows for frontal cortex (and occipital cortex for comparison) the evolution of gamma shape and scale parameters over all time windows. Even though data are not normalized within task blocks, a very clear effect on frontal cortex voluntary control can be observed which is absent for passive viewing. Thus, we do not find any evidence that inhibitory TMS over frontal cortex affects passive bistable viewing mechanisms. This is very informative in light of the strong TMS effect, targeting the exact same region in the same participants, on voluntary control.

\section{Frontal Cortex in Voluntary Control}

We hypothesized that, if frontal cortex were a cerebral source of voluntary control over bistable vision, inhibitory rTMS should increase nPD scores (reflecting fewer switches and thus reduced ability to make the percept switch frequently). In stark contrast to passive viewing, voluntary control was significantly affected by TMS. In the SFM trial immediately following rTMS, normalized PD was significantly above $100 \%$ for frontal stimulation ( $\mathrm{t} 9=2.90, \mathrm{P}<0.01$, uncorrected). As expected, $\mathrm{nPD}$ was no longer significantly above $100 \%$ in the second SFM trial after rTMS, suggesting time specificity of the TMS effects. Parietal TMS had more ambiguous effects, revealing only a trend on $\mathrm{nPD}(\mathrm{t} 9=1.39, \mathrm{P}<0.1$, uncorrected). Since no rigorous statistical effect could be shown here and no effect in distribution analyses (see below), we conservatively focus on the frontal region. (For additional comparisons and discussion, see Discussion and Supplementary Material.) There were no TMS effects on the lowlevel visual regions for either task (see Fig. 3C,D and Supplementary Material). To be precise: for the voluntary control condition, the statistical values of hMT and occipital cortex were t9 $=-0.54, \mathrm{P}=0.60$ and $\mathrm{t} 9=0.63, \mathrm{P}=0.55$, respectively. Note that the occipital and hMT regions might have yielded TMS effects on passive bistable vision with higher TMS intensities (since intensity was related to motor threshold instead of phosphene threshold - see Materials and Methods and Supplementary Material), so we should not conclude that they are not involved in bistable vision. In the current data set, these target regions can primarily serve as control regions for the frontal TMS effect on voluntary control.

Thus, whereas the lack of frontal TMS effects in passive viewing shows that the voluntary control frontal effects were task-specific, the lack of voluntary control 
TMS effects in early visual cortex regions shows that the frontal effects were regionspecific.

This was again confirmed by the gamma fit analyses. In the histogram of raw non-normalized PDs for frontal cortex and voluntary control (Fig. 4A: upper right), the naked eye can detect the shift in the distribution corresponding to the trials of interest (SFM trial 4, orange) as compared with control trial SFM trial 1. (The frontal SFM trial 4 histogram also contrasts strongly to the histograms of other TMS target sites: see Supplementary Figure S2.) The gamma curves representing these distributions (inset) reflect the same shift. Figure 4B shows a clear peak in gamma shape parameter and clear dip in gamma scale parameter, at the trials of interest, in the context of the other SFM trials. The $95 \%$ confidence intervals shading these curves suggest that the effect is consistent and reliable. Indeed, this is confirmed by the statistical comparison of the trials of interest within block (gamma fit of SFM4 is significantly different from SFM6: $\mathrm{P}<0.000$, corrected). For no other TMS site did this comparison yield significant TMS effects. Also between blocks, when comparing frontal TMS (SFM trial 4) in the voluntary control condition with the collapsed pre-TMS trials, there was a strong TMS effect on gamma fit $(\mathrm{P}<0.000$, corrected). Thus, again, the gamma distribution analyses support the frontal findings already presented above in nPD analyses, in analogous statistical comparisons.

In summary, while frontal TMS had no effects of any kind on the nPD or distribution of PDs for passive viewing, voluntary control of bistable perception was strongly affected.

\section{Discussion}

In the current study, we investigated the role of frontal cortex in passive bistable perception and voluntary control. We found clear evidence for functional relevance of frontal cortex in voluntary control. Thus, frontal cortex is a source of top-down modulation during controlled bistable vision. In contrast, we failed to find evidence for such top-down modulation when subjects engaged in passive viewing of the bistable SFM stimulus, where only spontaneous switches occurred.

\section{The Nature of Voluntary Control over Bistable Vision}

The effects obtained, or their direction, may be dependent on the nature of the voluntary control. Other types of voluntary control tasks should be investigated to evaluate potential similarities or differences to our results. Indeed, previous research suggests that the involvement of frontal cortex in voluntary control may be different for voluntarily inducing perceptual switches than, for example, for voluntarily maintaining 1 of the 2 percepts (Windmann et al. 2006; Pitts, Gavin, and Nerger 2008; Kornmeier et al. 2009). In our view, this suggests that the induction of switches by frontal cortex is a specific mechanism, rather than a general attention process. 
On the basis of our data, we cannot exclude the possibility that the frontal TMS protocol affected attention as a whole. A disruption of the attention system might have decreased participants' ability to focus on their task and thereby yield the obtained results. Thus, disruption of a general attention mechanism, rather than voluntary control specifically, constitutes a rival hypothesis to explain our data. However, recent research has shown that attention can affect passive bistable viewing switch rates (Paffen et al. 2006; Alais et al. 2010; Paffen and van der Stigchel 2010). Thus, if we disrupted attention, we might have expected some TMS effects on passive viewing as well. We found no such effects of any kind. Also, given the large effect size of approximately $40 \%$ increase in nPDs, and keeping in mind the cognitively central role and distributed nature of the attention network, it seems unlikely that our TMS was so strong as to affect the whole attention network to such effect. The attention system is a widespread network including both frontal and parietal regions, both stimulated in the current experiment. Our previous work did suggest that TMS over one node of a large network can affect the network as a whole (Sack et al. 2007; de Graaf, Jacobs, et al. 2009; de Graaf, Roebroeck, et al. 2010), but then TMS over the different network nodes had comparable effects (de Graaf, Jacobs, et al. 2009). In the current study, frontal rTMS had strong effects on voluntary control where parietal TMS did not. As a last consideration, a disruption of the attention system as a whole would likely affect all voluntary control tasks equally. Although we did not measure the effect of our TMS protocol on other voluntary control tasks, aforementioned studies did differentiate between these tasks in various paradigms.

Therefore, we propose that voluntary control over bistable vision, as measured by voluntarily induced perceptual switches, is a specialized mechanism (which may also partly explain why not all participants are equally successful at it; see also Borsellino et al. 1982; Liebert and Burk 1985; Struber and Stadler 1999; Struber et al. 2000; Pitts, Gavin, and Nerger 2008).

\section{Frontoparietal Cortices and Bistable Vision}

Using TMS, one should be careful to draw conclusions from null findings. In the "infinite parameter space," it is always possible that other TMS parameters would elicit different results. But in this particular study, there is tangible proof that TMS had neural effects on the frontal target site in the targeted subjects. Namely, stimulation of the exact same frontal region in the exact same subjects "did" reduce top-down modulation as implemented by conscious will (voluntary control), while it "did not" change passive switching behavior. That makes these latter null results very informative, as was outlined in our recent review on TMS null result interpretation guidelines (de Graaf and Sack 2011; next Chapter). The same cannot be said for parietal cortex, so we should be more conservative interpreting the passive viewing null findings there.

Three other recent studies investigated the role of parietal cortex in perceptual switching (Carmel et al. 2010; Kanai et al. 2010; Zaretskaya et al. 2010). However, as pointed out by Clifford (2010), rather than clarifying the role of parietal cortex, these 
studies reported opposite findings: Kanai et al. (2010) found decreased perceptual switching after inhibitory TMS, while Carmel et al. (2010) found increased perceptual switching after inhibitory TMS. Zaretskaya et al. (2010) in contrast used online TMS during task performance and found again decreased perceptual switching. Thus, the TMS protocols in these studies, and again in ours, differed (see also Clifford 2010). Second, while Kanai et al. (2010) used a bistable stimulus similar to ours, Carmel et al. (2010) and Zaretskaya et al. (2010) employed a binocular rivalry paradigm. These conflicting findings are not straightforward to reconcile, and the issue of parietal involvement in both passive bistable vision and voluntary control thus remains open (see Supplementary Material for further discussion of our parietal findings). In accordance with our recent guidelines (de Graaf and Sack 2011), we are careful not to draw strong conclusions on the basis of our parietal, hMT/V5, and occipital null results for both tasks. For frontal cortex, however, meaningful interpretations are certainly warranted.

To our knowledge, no TMS studies on frontal cortex in bistable vision or voluntary control thereof have yet been reported. We unambiguously found that the stimulated dorsolateral prefrontal cortex was functionally involved in the voluntary control task. This is in line with previous lesion (Windmann et al. 2006) and EEG (Pitts, Gavin, and Nerger 2008) research. In terms of effect size, for a TMS study the effects were impressively pronounced in our experience. It may thus be that the source of our voluntary control task is quite specific to dorsolateral prefrontal cortex (but see also Slotnick and Yantis 2005). Frontal TMS disruption of the ability to voluntarily control bistable vision supports the idea that frontal regions can instigate perceptual reconfiguration, in line with exploration/attention-based theories of perceptual switching (Leopold and Logothetis 1999; Rees 2004; Slotnick and Yantis 2005; Pitts, Nerger, and Davis 2007; Pitts, Gavin, and Nerger 2008; Sterzer et al. 2009). However, the same TMS protocol over the same region in the same participants did not have any effects on passive bistable vision. Intriguingly, this speaks against the suggestion that spontaneous perceptual switching may involve the exact same mechanism/pathway.

Despite our clear lack of frontal TMS effects on passive bistable vision, widespread activity changes have previously been reported for perceptual bistability in both fMRI studies (Lumer et al. 1998; Lumer and Rees 1999; Sterzer et al. 2002) and magnetoencephalography studies (Tononi et al. 1998; Srinivasan et al. 1999). Partly based on such studies, a causal role has been ascribed to frontoparietal regions by several authors (for references see above). Recent studies have questioned such a role, providing alternative explanations for the frontoparietal findings (Kamphuisen et al. 2008; Raemaekers et al. 2009). In our direct empirical investigation of frontal functional relevance using a virtual lesion approach, our findings speak against a causal role for frontal cortex in top-down modulation of bistable vision of the SFM stimulus, in so far as this modulation is automatic and employs the same regions/pathways as willed top-down modulation does.

It could still be that frontal cortex is causally involved in the induction of spontaneous perceptual switches, in 2 scenarios. Perhaps, automatic top-down modulation involves a different (e.g., more ventral) frontal region from the one currently stimulated (dorsolateral prefrontal cortex). Alternatively, automatic top-down 
modulation may involve the same regions but a different neural process from voluntary top-down modulation-one not easily disrupted with TMS or with the current TMS protocol. In either of these scenarios, we would nonetheless conclude that the kind of modulatory role frontal cortex plays in passive bistable vision seems fundamentally different from the kind of top-down modulation employed in voluntary control. Alternatively, perhaps dorsolateral prefrontal cortex is simply not causally involved in spontaneous perceptual switches. Instead, automatic perceptual switches could be induced in lower levels in the visual system but subsequently cause neural effects in frontal regions. A reevaluation of the representation of the outside world, even if not induced by frontal regions, would be highly relevant to many cognitive systems. Such spontaneous revisions of the visual input might therefore have salient effects in attentional/executive systems localized in prefrontal cortex. This would also explain the activation results in frontal cortex during perceptual switches. Evolutionarily speaking, it would even make sense to say that endogenous switches are more relevant than switches induced by stimulus changes (therefore leading to more frontal/parietal activation). After all, endogenous switches represent a form of correction of an earlier mistake: a new conclusion based on the same data.

\section{Conclusions}

We here identified a cerebral source of voluntary control over bistable perception, directly revealing a top-down modulation mechanism originating in frontal cortex. Subjects were substantially and significantly less able to voluntarily induce perceptual switches after inhibitory frontal TMS. This provides a neural basis for a score of psychophysical work revealing human capacity to voluntarily control bistable vision to an extent. Such a top-down mechanism had also been proposed for spontaneous perceptual switching during passive bistable vision. But the same "virtual lesion" in the same subjects that interfered with top-down modulation during voluntary control had no effects on perceptual switch rates during passive viewing. This calls into question the causal role of at least dorsolateral prefrontal cortex in triggering passive switches of conscious percept, even though it is causally involved in triggering "willed" switches. At any rate, it suggests that the same neural pathway is not involved in voluntarily or spontaneously instigated perceptual switches. 


\section{References}

Alais D, van Boxtel JJ, Parker A, van Ee R. 2010. Attending to auditory signals slows visual alternations in binocular rivalry. Vision Res. 50:929--935.

Amassian V, Mari Z, Sagliocco L, Hassan N, Maccabee P, Cracco JB, Cracco RQ, Bodis-Wollner I. 2008. Perception of phosphenes and flashed alphabetical characters is enhanced by single-pulse trans- cranial magnetic stimulation of anterior frontal lobe: the thalamic gate hypothesis. Perception. 37:375--388.

Borsellino A, Carlini F, Riani M, Tuccio MT, De Marco A, Penengo P, Trabucco A. 1982. Effects of visual angle on perspective reversal for ambiguous patterns. Perception. 11:263--273.

Brascamp JW, Kanai R, Walsh V, van Ee R. 2010. Human middle temporal cortex, perceptual bias, and perceptual memory for ambiguous three-dimensional motion. $\mathrm{J}$ Neurosci. 30:760--766.

Braunstein M. 1977. Perceived direction of rotation of simulated three- dimensional patterns. Percept Psychophys. 21:553--557.

Brouwer GJ, van Ee R. 2006. Endogenous influences on perceptual bistability depend on exogenous stimulus characteristics. Vision Res. 46:3393--3402.

Brouwer GJ, van Ee R. 2007. Visual cortex allows prediction of perceptual states during ambiguous structure-from-motion. J Neurosci. 27:1015--1023.

Carmel D, Walsh V, Lavie N, Rees G. 2010. Right parietal TMS shortens dominance durations in binocular rivalry. Curr Biol. 20:R799--R800. Chen X, He S. 2004. Local factors determine the stabilization of monocular ambiguous and binocular rivalry stimuli. Curr Biol. 14:1013--1017.

Clifford CW. 2010. Visual perception: ambiguity involving parietal cortex. Curr Biol. 20:R813--R815.

Corbetta M. 1998. Frontoparietal cortical networks for directing attention and the eye to visual locations: identical, independent, or overlapping neural systems? Proc Natl Acad Sci U S A. 95:831--838. Coull JT, Frith CD, Frackowiak RS, Grasby PM. 1996. A fronto-parietal network for rapid visual information processing: a PET study of sustained attention and working memory. Neuropsychologia.

34:1085--1095.

de Graaf TA, Jacobs C, Roebroeck A, Sack AT. 2009. FMRI effective connectivity and TMS chronometry: complementary accounts of causality in the visuospatial judgment network. PLoS One. 4:e8307.

de Graaf TA, Roebroeck A, Goebel R, Sack AT. 2010. Brain network dynamics underlying visuospatial judgment: an FMRI connectivity study. J Cogn Neurosci. 22:2012--2026.

de Graaf TA, Sack AT. 2011. Null results in TMS: from absence of evidence to evidence of absence. Neurosci Biobehav Rev. 35:871--877.

Frost MA, Goebel R. 2009. Creating functional probabilistic maps using structurally and functionally driven multi-subject alignment. Neuro- image. 47.

Haynes JD, Deichmann R, Rees G. 2005. Eye-specific effects of binocular rivalry in the human lateral geniculate nucleus. Nature. 438: 496--499. 
Hol K, Koene A, van Ee R. 2003. Attention-biased multi-stable surface perception in three-dimensional structure-from-motion. J Vis. 3:486--498.

Horlitz KL, O’Leary A. 1993. Satiation or availability? Effects of attention, memory, and imagery on the perception of ambiguous figures. Percept Psychophys. 53:668--681.

Hsieh PJ, Caplovitz GP, Tse PU. 2006. Bistable illusory rebound motion: event-related functional magnetic resonance imaging of perceptual states and switches. Neuroimage. 32:728--739.

Hsieh PJ, Tse PU. 2009. Microsaccade rate varies with subjective visibility during motion-induced blindness. PLoS One. 4:e5163. Hsieh PJ, Tse PU. 2010. "Brainreading" of perceived colors reveals

a feature mixing mechanism underlying perceptual filling-in in cortical area V1. Hum Brain Mapp. 31(9):1395--1407.

Inui T, Tanaka S, Okada T, Nishizawa S, Katayama M, Konishi J. 2000. Neural substrates for depth perception of the Necker cube; a functional magnetic resonance imaging study in human subjects.

Neurosci Lett. 282:145--148.

Kamphuisen A, Bauer M, van Ee R. 2008. No evidence for widespread synchronized networks in binocular rivalry: MEG frequency tagging entrains primarily early visual cortex. J Vis. 8(4):1--8.

Kanai R, Bahrami B, Rees G. 2010. Human parietal cortex structure predicts individual differences in perceptual rivalry. Curr Biol. 20(18):1626--1630.

Kim CY, Blake R. 2005. Psychophysical magic: rendering the visible “invisible”. Trends Cogn Sci. 9:381--388.

Kleinschmidt A, Buchel C, Zeki S, Frackowiak RS. 1998. Human brain activity during spontaneously reversing perception of ambiguous figures. Proc Biol Sci. 265:2427--2433.

Klink PC, van Ee R, Nijs MM, Brouwer GJ, Noest AJ, van Wezel RJ. 2008.

Early interactions between neuronal adaptation and voluntary control determine perceptual choices in bistable vision. J Vis. 8(16):11--18.

Kornmeier J, Hein CM, Bach M. 2009. Multistable perception: when bottom-up and top-down coincide. Brain Cogn. 69:138--147.

Lee SH, Blake R. 2002. V1 activity is reduced during binocular rivalry. J Vis. 2:618-626.

Lee SH, Blake R, Heeger DJ. 2005. Traveling waves of activity in primary visual cortex during binocular rivalry. Nat Neurosci. 8:22--23.

Leopold DA, Logothetis NK. 1999. Multistable phenomena: changing views in perception. Trends Cogn Sci. 3:254--264.

Liebert RM, Burk B. 1985. Voluntary control of reversible figures. Percept Mot Skills. 61:1307--1310.

Lumer ED, Friston KJ, Rees G. 1998. Neural correlates of perceptual rivalry in the human brain. Science. 280:1930--1934.

Lumer ED, Rees G. 1999. Covariation of activity in visual and prefrontal cortex associated with subjective visual perception. Proc Natl Acad Sci U S A. 96:1669--1673. Meng M, Remus DA, Tong F. 2005. Filling-in of visual phantoms in the human brain. Nat Neurosci. 8:1248--1254. 
Meng M, Tong F. 2004. Can attention selectively bias bistable perception? Differences between binocular rivalry and ambiguous figures. J Vis. 4:539--551.

Moutoussis K, Keliris G, Kourtzi Z, Logothetis N. 2005. A binocular rivalry study of motion perception in the human brain. Vision Res. 45:2231--2243.

Naghavi HR, Nyberg L. 2005. Common fronto-parietal activity in attention, memory, and consciousness: shared demands on in- tegration? Conscious Cogn. 14:390--425.

Nobre AC, Coull JT, Frith CD, Mesulam MM. 1999. Orbitofrontal cortex is activated during breaches of expectation in tasks of visual attention. Nat Neurosci. 2:11--12.

Paffen CL, Alais D, Verstraten FA. 2006. Attention speeds binocular rivalry. Psychol Sci. 17:752--756.

Paffen CL, Van der Stigchel S. 2010. Shifting spatial attention makes you flip: exogenous visual attention triggers perceptual alternations during binocular rivalry. Atten Percept Psychophys. 72:1237--1243.

Pelton LH, Solley CM. 1968. Acceleration of reversals of a Necker cube. Am J Psychol. 81:585--588.

Pessoa L, Kastner S, Ungerleider LG. 2003. Neuroimaging studies of attention: from modulation of sensory processing to top-down control. J Neurosci. 23:3990--3998.

Pitts MA, Gavin WJ, Nerger JL. 2008. Early top-down influences on bistable perception revealed by event-related potentials. Brain Cogn. 67:11--24.

Pitts MA, Nerger JL, Davis TJ. 2007. Electrophysiological correlates of perceptual reversals for three different types of multistable images. J Vis. 7:6.

Polonsky A, Blake R, Braun J, Heeger DJ. 2000. Neuronal activity in human primary visual cortex correlates with perception during binocular rivalry. Nat Neurosci. 3:1153-1159.

Raemaekers M, van der Schaaf ME, van Ee R, van Wezel RJ. 2009. Widespread fMRI activity differences between perceptual states in visual rivalry are correlated with differences in observer biases. Brain Res. 1252:161--171.

Rees G. 2004. Neural correlates of visual consciousness in humans. In: Gazzaniga MS, editor. The Cognitive Neurosciences III. 3rd ed. Cambridge (MA): MIT Press. p. 1173-1188.

Rees G, Kreiman G, Koch C. 2002. Neural correlates of consciousness in humans. Nat Rev Neurosci. 3:261--270.

Rossi S, Hallett M, Rossini PM, Pascual-Leone A. 2009. Safety, ethical considerations, and application guidelines for the use of transcranial magnetic stimulation in clinical practice and research. Clin Neuro- physiol. 120:2008--2039.

Sack AT, Kohler A, Bestmann S, Linden DE, Dechent P, Goebel R, Baudewig J. 2007. Imaging the brain activity changes underlying impaired visuospatial judgments: simultaneous FMRI, TMS, and behavioral studies. Cereb Cortex. 17:2841--2852.

Schoth F, Waberski TD, Krings T, Gobbele R, Buchner H. 2007. Cerebral processing of spontaneous reversals of the rotating Necker cube. Neuroreport. 18:1335--1338.

Slotnick SD, Yantis S. 2005. Common neural substrates for the control and effects of visual attention and perceptual bistability. Brain Res Cogn Brain Res. 24:97--108.

Srinivasan R, Russell DP, Edelman GM, Tononi G. 1999. Increased synchronization of neuromagnetic responses during conscious perception. J Neurosci. 19:5435--5448. 
Sterzer P, Kleinschmidt A, Rees G. 2009. The neural bases of multistable perception. Trends Cogn Sci. 13:310--318.

Sterzer P, Rees G. 2008. A neural basis for percept stabilization in binocular rivalry. J Cogn Neurosci. 20:389--399.

Sterzer P, Russ MO, Preibisch C, Kleinschmidt A. 2002. Neural correlates of spontaneous direction reversals in ambiguous apparent visual motion. Neuroimage. 15:908--916.

Stokes MG, Chambers CD, Gould IC, English T, McNaught E, McDonald O, Mattingley JB. 2007. Distance-adjusted motor thresh- old for transcranial magnetic stimulation. Clin Neurophysiol. 118:1617--1625.

Stokes MG, Chambers CD, Gould IC, Henderson TR, Janko NE, Allen NB, Mattingley JB. 2005. Simple metric for scaling motor threshold based on scalp-cortex distance: application to studies using transcranial magnetic stimulation. J Neurophysiol. 94:4520$-4527$.

Struber D, Basar-Eroglu C, Hoff E, Stadler M. 2000. Reversal-rate dependent differences in the EEG gamma-band during multistable visual perception. Int $\mathrm{J}$ Psychophysiol. 38:243--252.

Struber D, Stadler M. 1999. Differences in top-down influences on the reversal rate of different categories of reversible figures. Perception. 28:1185--1196.

Tong F, Engel SA. 2001. Interocular rivalry revealed in the human cortical blind-spot representation. Nature. 411:195--199.

Tong F, Nakayama K, Vaughan JT, Kanwisher N. 1998. Binocular rivalry and visual awareness in human extrastriate cortex. Neuron. 21:753--759.

Tononi G, Srinivasan R, Russell DP, Edelman GM. 1998. Investigating neural correlates of conscious perception by frequency-tagged neuromagnetic responses. Proc Natl Acad Sci U S A. 95:3198--3203.

Toppino TC. 2003. Reversible-figure perception: mechanisms of intentional control. Percept Psychophys. 65:1285--1295.

van Ee R, van Dam LC, Brouwer GJ. 2005. Voluntary control and the dynamics of perceptual bi-stability. Vision Res. 45:41--55.

Wallach H, O’Connel D. 1953. The kinetic depth effect. J Exp Psychol. 45:205--217.

Wassermann EM, Epstein CM, Ziemann U, Walsh V, Paus T, Lisanby SH. 2008. The Oxford Handbook of Transcranial Stimulation. New York: Oxford University Press.

Windmann S, Wehrmann M, Calabrese P, Gunturkun O. 2006. Role of the prefrontal cortex in attentional control over bistable vision. J Cogn Neurosci. 18:456--471.

Wunderlich K, Schneider KA, Kastner S. 2005. Neural correlates of binocular rivalry in the human lateral geniculate nucleus. Nat Neurosci. 8:1595--1602.

Zaretskaya N, Thielscher A, Logothetis NK, Bartels A. 2010. Disrupting parietal function prolongs dominance durations in binocular rivalry. Curr Biol. 20(23):2106-2111. 


\section{Supplementary Material}

Voluntary control versus passive viewing without TMS

We first checked whether participants were indeed able to perform the voluntary control task. For the ten subjects who finished all sessions, the percept durations (PD) averaged for both no-TMS blocks (pre-TMS and late post-TMS blocks) were compared, to ensure significantly longer PDs during passive viewing. This was so for all TMS stimulation sites. For the frontal and parietal sessions which were of primary interest: parietal PDs were for passive viewing (average of individual means [SD] in seconds: 7.50[1.82]) and for voluntary control (3.92[2.06]) which constitutes a statistically significant difference ( $\mathrm{t}[9]=6.58, \mathrm{P}<0.001$, one-tailed uncorr.); frontal PDs were for passive viewing $(8.50[4.13])$ and for voluntary control $(3.55[1.97])$, significantly different $(\mathrm{t}[9]=4.38$, $\mathrm{P}=0.001$, one-tailed uncorr.). We thus replicated the psychophysical results found by Brouwer and van Ee (2006), showing that the current stimulus and tasks made conscious voluntary control over bistable vision possible. To inspect absolute PDs for all sites and all tasks, we refer to Table S1. To inspect gamma parameters for all sites and tasks, we refer to Table S2. Percept durations for individual participants were normalized around their individual means, all data points were then fitted to a gamma distribution. Table S2 presents both shape and scale parameters describing the resulting fits for all sites, time windows, and tasks.

\begin{tabular}{|c|c|c|c|c|c|c|c|c|c|c|}
\hline \multirow[b]{3}{*}{ Parietal } & \multirow[b]{3}{*}{$\mathrm{P}$} & \multicolumn{2}{|l|}{ Pre-TMS } & \multirow[b]{2}{*}{ SFM3 } & \multicolumn{3}{|c|}{ immediate Post-TMS } & \multicolumn{3}{|c|}{ late Post-TMS } \\
\hline & & SFM1 & SFM2 & & \multirow{2}{*}{$\begin{array}{l}\text { SFM4 } \\
7,19\end{array}$} & \multirow{2}{*}{$\frac{\text { SFM5 }}{6,49}$} & \multirow{2}{*}{$\frac{\text { SFM6 }}{7,87}$} & \multirow{2}{*}{$\frac{\text { SFM7 }}{6,13}$} & \multirow{2}{*}{$\frac{\text { SFM8 }}{8,05}$} & \multirow{2}{*}{$\frac{\text { SFM9 }}{9,42}$} \\
\hline & & 6,68 & 7,14 & 7,59 & & & & & & \\
\hline & V & 3,83 & 4,16 & 4,44 & 4,06 & 3,10 & 3,28 & 3,58 & 3,62 & 3,88 \\
\hline \multirow[t]{2}{*}{ Frontal } & $\mathrm{P}$ & 7,53 & 8,45 & 7,52 & 7,94 & 7,63 & 8,42 & 8,16 & 10,27 & 9,09 \\
\hline & V & 3,41 & 3,48 & 3,80 & 4,36 & 3,27 & 3,10 & 3,61 & 3,45 & 3,53 \\
\hline \multirow[t]{2}{*}{ hMT/V5 } & $\mathrm{P}$ & 7,91 & 7,78 & 7,67 & 6,69 & 6,54 & 6,79 & 6,79 & 8,84 & 7,76 \\
\hline & V & 2,40 & 2,62 & 2,95 & 3,15 & 2,75 & 2,70 & 2,54 & 2,53 & 2,98 \\
\hline \multirow[t]{2}{*}{ Occipital } & $\mathrm{P}$ & 5,89 & 6,50 & 6,50 & 6,65 & 6,75 & 6,96 & 6,37 & 7,18 & 6,81 \\
\hline & V & 2,74 & 2,85 & 2,75 & 2,74 & 2,44 & 2,71 & 2,65 & 2,99 & 2,96 \\
\hline
\end{tabular}

\section{Further analyses on TMS effects}

Although we tested nPD against $100 \%$, our planned comparison, due to the normalization procedure this was a within-task-block comparison. Many task blocks in voluntary control show a rising trend from the first to the third SFM trial (the first SFM trial, normalized, was below $100 \%$ for six out of the eight control clusters - see Figure 3 ). This is likely due to fatigue, considering the effort and concentration required for continuous switching. The fact that the trend is reversed immediately after TMS over frontal cortex strengthens our conclusions. Due to variability between task-blocks we believe that our normalization procedure was justified, but we checked nonetheless whether a one-step normalization procedure - that is not within task-block - would yield the same effects. This means we normalized all analyzed time windows to one fixed baseline value per subject. For this, we normalized each SFM trial post-TMS 
(SFM trials 4 to 9) to the pre-TMS baseline percept duration. Figure S1 shows the results of this normalization procedure (see Supplementary Methods for more detail). Here, only on frontal cortex, and only on voluntary control, did TMS have effects surpassing our statistical threshold. The late-post-TMS trials are also normalized to the pre-TMS trials, for completeness sake. It seems that some of these later trials have nPDs higher than $100 \%$. However, these comparisons are not very meaningful, since the normalization now is based on pre-TMS trials at the beginning of the experiment when arousal and attention were yet high and fatigue low. This is one of the reasons why we favoured within-block normalization as presented in the main text.

Importantly, putting these graphs and Figures 3 and 4 from the main text together, it becomes clear that the normalization procedure selected has no influence on whether frontal cortex is affected in passive and voluntary control. However, while a trend of a parietal TMS effect can be seen in nPDs, the gamma fit analyses speak against any parietal effect. In no comparison was there even a trend of TMS effects on either passive viewing or voluntary control for the parietal cortex. Figure S2 displays the voluntary control histograms of non-normalized raw percept durations for the trialof-interest (which might have revealed TMS effects) and a control trial (first pre-TMS baseline trial). This figure is analogous to, and complements, main Figure 4A - where the frontal cortex histogram was presented. No effects on the distribution of percept duration for any TMS site is apparent, in stark contrast to frontal cortex (Figure 4A upper right). So, here parietal TMS seems to have no effects, which is supported statistically (see main text). A possible explanation of the trend in parietal TMS on voluntary control might be inferred from Table S1. Looking at the raw data in Table S1, where non-normalized percept durations are shown - it becomes clear that normalization procedures that compare, somehow, SFM trials 4 to SFM trials 5 or 6 show a parietal effect. But this is more because of a dip in average percept duration of trials 5 and 6 than a rise in percept duration in trial 4 , immediately after TMS. We cannot be sure whether this is because the three trials after TMS subjects are aroused and more motivated (since 'this must be an important task block - right after TMS'), and that the relatively high PD in trial 4 is due to TMS, or whether the decrease of PD in trials 5 and 6 is random. But the normalization procedure selected has no effect

\begin{tabular}{|c|c|c|c|c|c|c|c|c|c|c|c|}
\hline \multirow[b]{3}{*}{ Shape } & \multirow[b]{3}{*}{ Parietal } & \multirow[b]{3}{*}{$P$} & \multicolumn{3}{|l|}{ Pre-TMS } & \multicolumn{3}{|c|}{ immediate Post-TMS } & \multicolumn{3}{|c|}{ late Post-TMS } \\
\hline & & & SFM1 & SFM2 & SFM3 & SFM4 & SFM5 & SFM6 & SFM7 & SFM8 & SFM9 \\
\hline & & & 0,42 & 0,50 & 0,43 & 0,40 & 0,37 & 0,51 & 0,51 & 0,57 & 0,50 \\
\hline \multirow{7}{*}{ Parameter } & & v & 0,18 & 0,22 & 0,19 & 0,19 & 0,17 & 0,17 & 0,18 & 0,18 & 0,24 \\
\hline & Frontal & $P$ & 0,33 & 0,36 & 0,32 & 0,43 & 0,39 & 0,33 & 0,37 & 0,35 & 0,36 \\
\hline & & v & 0,18 & 0,17 & 0,23 & 0,31 & 0,19 & 0,16 & 0,21 & 0,17 & 0,24 \\
\hline & hMT/V5 & $\mathrm{P}$ & 0,33 & 0,37 & 0,34 & 0,37 & 0,28 & 0,36 & 0,37 & 0,45 & 0,33 \\
\hline & & V & 0,15 & 0,14 & 0,14 & 0,21 & 0,18 & 0,20 & 0,17 & 0,15 & 0,17 \\
\hline & Occipital & $\mathrm{P}$ & 0,33 & 0,28 & 0,26 & 0,38 & 0,25 & 0,27 & 0,32 & 0,33 & 0,29 \\
\hline & & V & 0,17 & 0,17 & 0,15 & 0,15 & 0,13 & 0,15 & 0,16 & 0,15 & 0,14 \\
\hline Scale & Parietal & $P$ & 2,36 & 2,02 & 2,34 & 2,52 & 2,74 & 1,96 & 1,94 & 1,76 & 1,98 \\
\hline \multirow{7}{*}{ Parameter } & & $\mathrm{v}$ & 5,47 & 4,60 & 5,35 & 5,28 & 5,85 & 5,89 & 5,65 & 5,42 & 4,19 \\
\hline & Frontal & $P$ & 3,03 & 2,81 & 3,17 & 2,31 & 2,59 & 3,07 & 2,73 & 2,89 & 2,75 \\
\hline & & V & 5,56 & 5,80 & 4,39 & 3,26 & 5,27 & 6,32 & 4,66 & 6,04 & 4,22 \\
\hline & $\mathrm{hMT} / \mathrm{V} 5$ & $P$ & 3,00 & 2,71 & 2,98 & 2,70 & 3,55 & 2,75 & 2,68 & 2,21 & 3,00 \\
\hline & & V & 6,85 & 7,34 & 7,09 & 4,86 & 5,58 & 5,03 & 5,84 & 6,47 & 5,96 \\
\hline & Occipital & $\mathrm{P}$ & 3,04 & 3,57 & 3,91 & 2,63 & 3,95 & 3,66 & 3,13 & 2,99 & 3,50 \\
\hline & & V & 5,91 & 5,82 & 6,62 & 6,69 & 7,54 & 6,79 & 6,27 & 6,48 & 7,03 \\
\hline
\end{tabular}


whatsoever on any of the frontal results, passive viewing results, or early visual regions results. Conservatively, we therefore draw conclusions mainly concerning the frontal region while being extremely careful concerning parietal cortex. Concerning passive viewing in parietal cortex, we discuss in the main text three new studies recently published (Carmel et al. 2010; Kanai et al. 2010; Zaretskaya et al. 2010). While these studies all suggest that a region within parietal cortex is involved in passive bistable viewing, their findings are difficult to reconcile. As presented in the main text, different paradigms and TMS protocols were used. Thus, the precise bistable vision paradigm and the precise regiment of neural stimulation with parietal TMS seem to affect what happens with passive bistable viewing. Moreover, Zaretskaya et al. (2010) showed also that TMS over one parietal region (inferior parietal sulcus) did have effects on passive viewing behaviour, while TMS over a neighboring parietal region (superior parietal lobule) did not - so the spatial layout of parietal involvement merits further investigation as well. All things considered, the role of parietal cortex remains unclear. We therefore present our parietal results as they are, to complement the body of evidence recently accumulated, without drawing strong conclusions about this region on the basis of our data alone. In contrast, as we discuss in the main text, in accordance with our recent guidelines (de Graaf and Sack 2011) our dataset does afford several interesting conclusions about the role of the stimulated prefrontal region in bistable vision and voluntary control (see Discussion of main text).

Considerations of Eye Movements, Spreading Excitation, and Broad Implications

We here would like to address the possible role of eye movements in our data and interpretations, and discuss some implications of our data in the broader frame of consciousness research.

First, eye movements are unlikely to have contributed importantly to our results. TMS effects on eye movements are not uncommon, but only if eye movement preparation or execution falls within a relatively narrow time window (up to $150 \mathrm{~ms}$ ) around the TMS pulse (e.g. Gagnon et al. 2006; Drew and van Donkelaar 2007; Walker et al., 2009). In our case, visual stimulation did not start until several seconds after TMS was applied. This renders a direct perceptual effect of TMS via eye movements unlikely. Moreover, whether eye movement modulations in general influence perception of our stimulus is questionable. Previous studies have found no consistent relationship between eye movements and perception of ambiguous stimuli identical or very similar to ours (Brouwer and van Ee 2006; 2007; Klink et al. 2008), and a recent study that did observe such a relation in a different stimulus (using ambiguous apparent motion) showed that, nevertheless, manipulating eye movements did not influence perception (Laubrock et al. 2007).

Second, bistable vision is a popular research paradigm, both in its own right and in the larger context of consciousness research. A comprehensive discussion of the literature on neural correlates of consciousness is beyond the scope of the current paper. But we wish to point out that frontoparietal activations have been reported in studies using very different consciousness paradigms, such as masking (e.g. Dehaene et al. 2001) or attentional blinks (e.g. Kranczioch et al., 2005). As a result, frontal and/or parietal regions have been ascribed various important roles in the establishment of 
conscious vision. But fMRI evidence in itself seems to us insufficient to establish such a functional role. As mentioned, it is also possible that the establishment of a conscious percept has repercussions throughout the brain, causing widespread activations rather than being caused by them. Our results are at the very least compatible with this latter scenario, and thus may have a bearing on the larger questions of conscious vision and how it is established. TMS as a method seems suitable to probe these issues further.

\section{Supplementary Methods}

\begin{tabular}{|c|c|c|c|c|c|c|c|c|c|c|c|c|}
\hline & Frontal & & & Parietal & & & hMTN5 & & & Occipital & & \\
\hline Subject & $\begin{array}{ll}X & Y \\
\end{array}$ & $z$ & & $\mathrm{x}$ & z & & $x$ & z & & $\begin{array}{ll}X & Y\end{array}$ & z & \\
\hline 1 & 27 & 33 & 44 & 26 & -66 & 47 & 43 & -79 & -2 & 0 & -97 & 11 \\
\hline 2 & 22 & 38 & 33 & 18 & -64 & 58 & 45 & -77 & -3 & 0 & -95 & 8 \\
\hline 3 & 29 & 26 & 49 & 35 & -74 & 42 & 50 & -70 & 0 & 0 & -101 & 14 \\
\hline 4 & 23 & 24 & 43 & 31 & -74 & 38 & 40 & -68 & -1 & 0 & -89 & 7 \\
\hline 5 & 20 & 11 & 44 & 19 & -58 & 46 & 37 & .74 & 2 & 0 & -94 & 25 \\
\hline 6 & 24 & 34 & 33 & 20 & -82 & 39 & 34 & -74 & 10 & 0 & $\begin{array}{l}-102 \\
-102\end{array}$ & 9 \\
\hline 7 & 23 & 14 & 42 & 23 & -64 & 45 & 41 & -76 & -5 & 0 & -101 & -8 \\
\hline 8 & 18 & 42 & 47 & 32 & -70 & 41 & 47 & -71 & 5 & 0 & -98 & 3 \\
\hline 9 & 38 & 22 & 47 & 44 & -68 & 37 & 54 & -63 & 4 & 0 & -93 & 21 \\
\hline 10 & 24 & 23 & 47 & 21 & -86 & 31 & 44 & -66 & 1 & 0 & -94 & 8 \\
\hline mean & 24,8 & 26,7 & 42,9 & 26,9 & $-70,6$ & 42,4 & 43,5 & $-71,8$ & 1,1 & 0,0 & $-96,4$ & 9,8 \\
\hline average deviation & 3,9 & 8,0 & 4,1 & 6,9 & 6,7 & 5,3 & 4,5 & 4,2 & 3,3 & 0,0 & 3,4 & 6,4 \\
\hline standard deviation & 5,6 & 10,0 & 5,6 & 8,4 & 8,6 & 7,3 & 5,9 & 5,1 & 4,4 & 0,0 & 4,2 & 9,2 \\
\hline$\underline{\text { standard error }}$ & 1,8 & 3,2 & 1,8 & 2,7 & 2,7 & 2,3 & 1,9 & 1,6 & 1.4 & 0,0 & 1,3 & 2,9 \\
\hline
\end{tabular}

Targeted TMS sites - individual coordinates and variability

\section{Outlier removal}

Individual normalized percept durations above $220 \%$ were considered outliers if removal of these datapoints proved to substantially alter the pattern of effects. This happened twice (on time window 8 of hMT - passive, on time window 4 of hMT voluntary control, where an $\mathrm{nPD}$ of $250 \%$ in one participant inflated the group mean by nearly $15 \% \mathrm{nPD}$ ). In graphs these outliers were left out, in analyses they were replaced with the group average. Note that our group effect on frontal cortex - voluntary control was not thus inflated by any individual value and included no outliers. We did not have to eliminate any outliers in that condition in spite of the very high group average nPD.

\section{Normalization procedures:}

We applied two normalization procedures. The first underlies Figure 3 and was presented in the main Methods. This normalization procedure normalized PDs within task-blocks. Considering the differences in practice, fatigue, motivation, etc., this was our normalization procedure of choice. However, to ensure the effects were no artefact of the normalization procedure selected, we also normalized the data between task blocks. This second normalization procedure involved normalization to the average percept duration across all pre-TMS baseline trials. This normalization procedure was added because it only involved one normalization step of all time windows under analysis (namely the three post-TMS, and the three late-post-TMS), which might be preferred by some. In this normalization we took the average percept duration per subject in the three pre-TMS SFM trials - per session (site). The average percept 
duration of SFM trials 4, 5, 6, 7, 8, 9 were then divided by this average, and multiplied by $100 \%$. This resulted in six ratios, or normalized percept durations, per subject per site, of which the first was the ratio of interest (highlighted in Figure S1) and was compared to the other five ratios with one-tailed t-tests. Since statistical comparisons became many, in this analysis, a more conservative threshold of 0.01 was adopted.

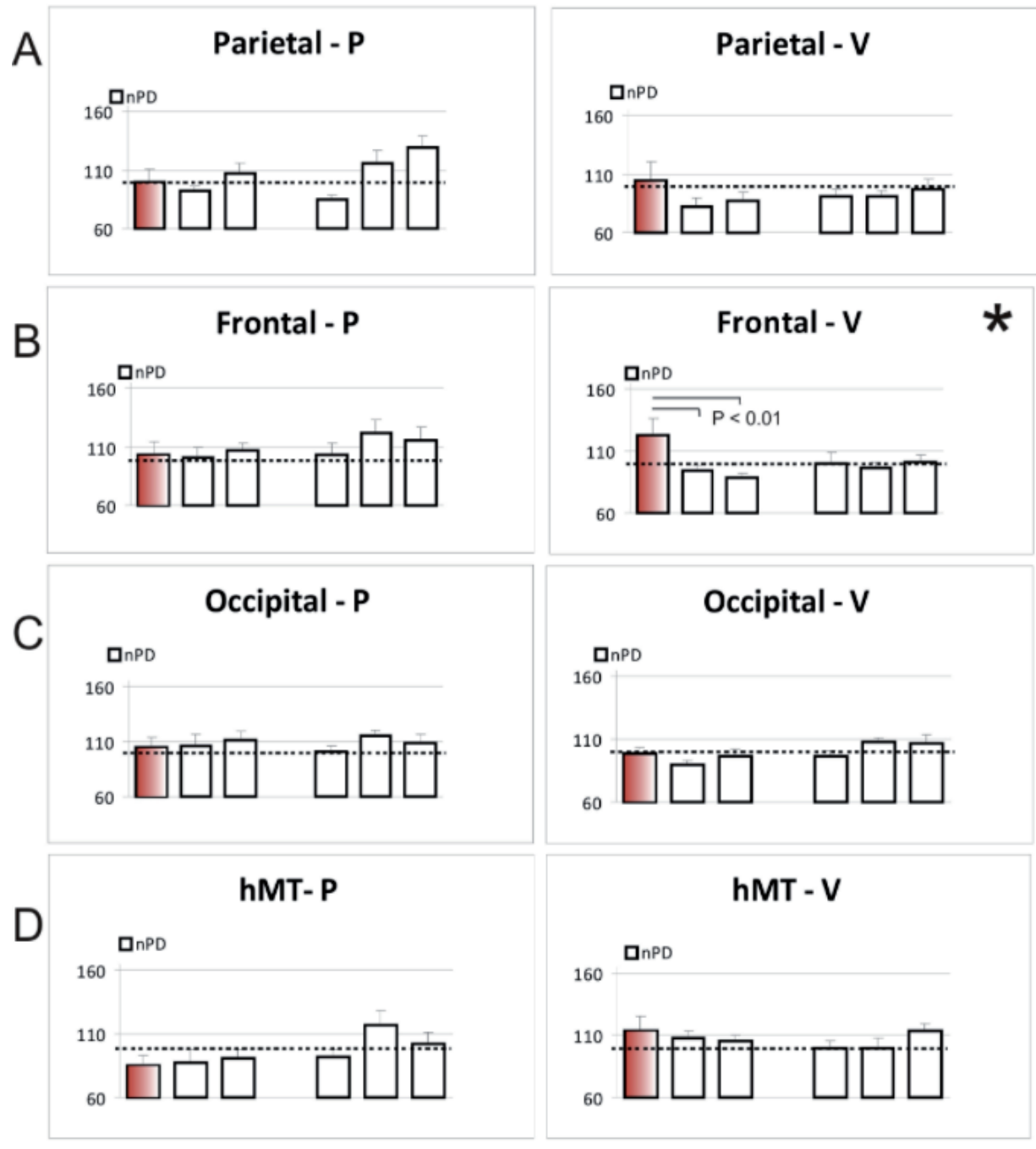

Figure S1: results from single-step normalization to average pre-TMS baseline PDs Indicated with asterisks are sites/tasks where the time window of interest ratio (first time window after TMS) was significantly different from (at least one of) the other five time windows - all normalized to the same pre-TMS percept duration average. This was the case for the voluntary control task in frontal TMS. In all other sites/tasks, this ratio was not significantly different in any comparison. A. parietal TMS. B. frontal TMS. C. hMT/V5 TMS. D. Occipital TMS. P=Passive task. V=Voluntary control task. 

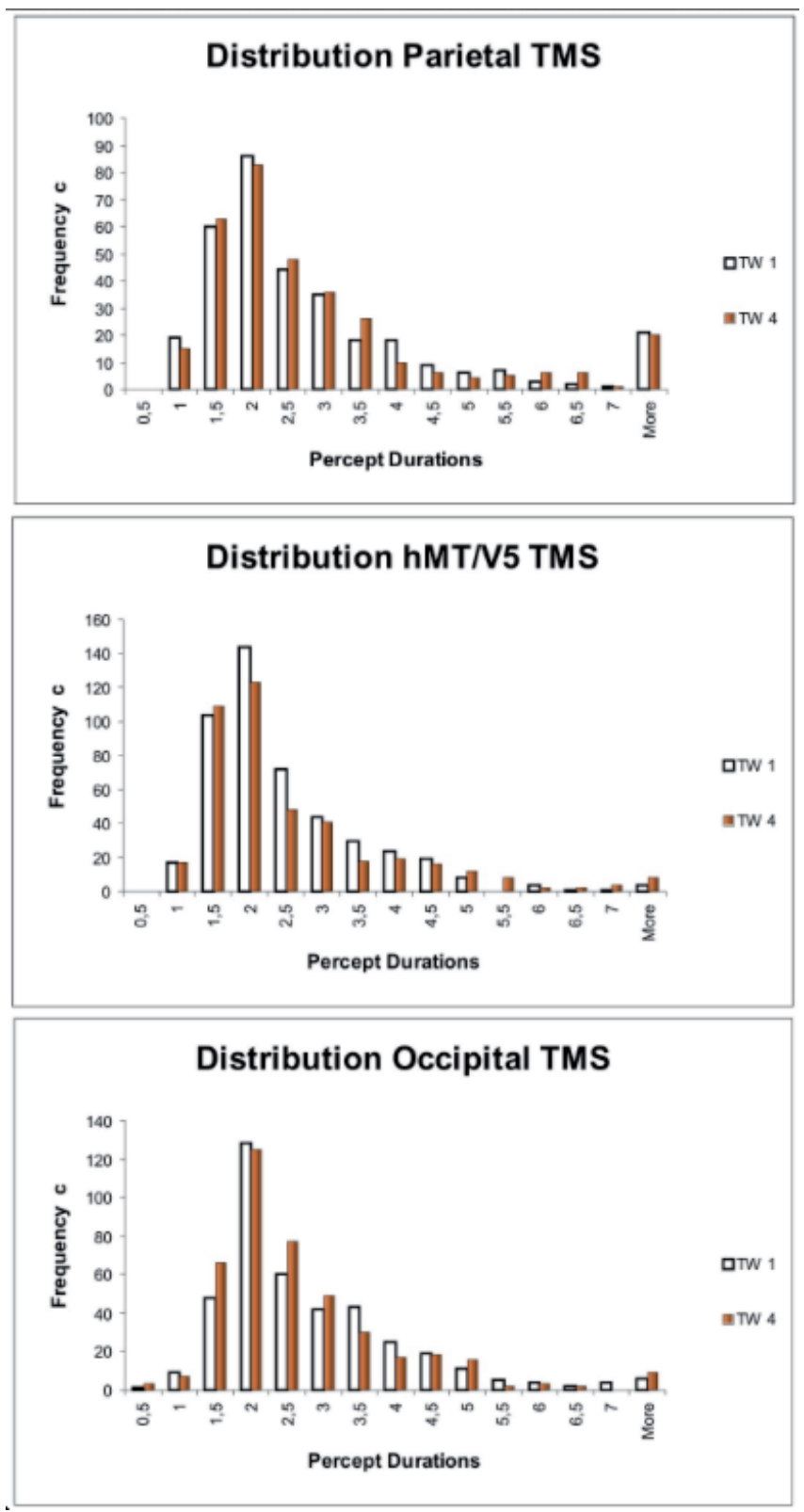

Figure S2:

Distributions of percept durations during voluntary control, for parietal, occipital, and hMT/V5 regions. Orange histogram represents the trialof-interest, which shows no difference in these regions to the white control histogram (representing the first pre-TMS baseline trial). This is in stark contrast to frontal TMS - see main Figure 4A 


\section{Supplementary References}

Brouwer GJ, van Ee R. (2006). Endogenous influences on perceptual bistability depend on exogenous stimulus characteristics. Vision Res. 46: 3393-3402.

Brouwer GJ, van Ee R. (2007). Visual cortex allows prediction of perceptual states during ambiguous structure-from-motion. J Neurosci. 27: 1015-1023.

Dehaene S, Naccache L, Cohen L, Bihan DL, Mangin JF, et al. (2001). Cerebral mechanisms of word masking and unconscious repetition priming. Nat Neurosci 4: 752758.

Drew AS, van Donkelaar P. (2007). The contribution of the human FEF and SEF to smooth pursuit initiation. Cereb Cortex 17: 2618-2624.

Gagnon D, Paus T, Grosbras MH, Pike GB, O'Driscoll GA. (2006). Transcranial magnetic stimulation of frontal oculomotor regions during smooth pursuit. J Neurosci 26: 458-466.

Klink PC, van Ee R, Nijs MM, Brouwer GJ, Noest AJ, et al. (2008). Early interactions between neuronal adaptation and voluntary control determine perceptual choices in bistable vision. J Vis 8: 16 11-18.

Kranczioch C, Debener S, Schwarzbach J, Goebel R, Engel AK. (2005). Neural correlates of conscious perception in the attentional blink. Neuroimage 24: 704-714.

Laubrock J, Engbert R, Kliegl R. (2008). Fixational eye movements predict the perceived direction of ambiguous apparent motion. J Vis 8: 13 11-17.

Walker R, Techawachirakul P, Haggard P. (2009). Frontal eye field stimulation modulates the balance of salience between target and distractors. Brain Res 1270: 5463. 


\title{
III. 2
}

\section{Null Results in TMS: from absence of}

\author{
evidence to evidence of absence
}

Related publication(s):

De Graaf, TA, Sack, AT (2011). Null results in TMS: from absence of evidence to evidence of absence. Neuroscience and Biobehavioral Reviews. 5: 871-877 


\begin{abstract}
It is always difficult to interpret null results. But as a research method, transcranial magnetic stimulation (TMS) has so many degrees of freedom that null results are often dismissed as meaningless. We feel that this may be unnecessary, if not counterproductive. Null results seem to inherently fulfill an important role in brain mapping. In fact, without null results, neuroimaging as an enterprise would not make sense. We argue that null results are similarly important in TMS research. By itself, neuroimaging research leaves room for doubt concerning whether or not an activated region is actually necessary for intact task performance. Interference methods such as TMS can therefore complement brain research by testing the functional relevance of that region. However, if then only positive TMS results are taken seriously, the brain interference paradigm seems less informative than promised. But how can null results inform us if they only constitute absence of evidence? We suggest that three main arguments contravene interpretation of null results in TMS. These we call the localization argument, the neural efficacy argument, and the power argument. We proceed to discuss in turn how, and under which conditions, each of these arguments may be nuanced. These considerations lead us to value null results along a gradient of meaningfulness, rather than a dichotomy. This perspective may open up a new range of TMS applications, where research questions about the lack of functional relevance of a particular brain region become valid. In this context we make specific recommendations on experimentation and interpretation. We propose that it is often not only meaningful to interpret null results, but also useful to make such findings available to the community, especially now that improved methods and an expanded knowledge base make null results more interpretable than they have been in the past.
\end{abstract}




\section{Introduction}

Absence of evidence is not evidence of absence. This adage summarizes a popular epistemological opinion on null results in many branches of science. If you do not see something, that does not mean it is not there. It certainly sounds reasonable, but this postulate raises a serious conundrum: what if you want to show that something does not exist? When can you conclude that something is not there?

In this article, we discuss how null results are regarded and processed in human brain science; in particular in labs using transcranial magnetic stimulation (TMS). This focus arises from our feeling that TMS null results are often considered to be more meaningless than is strictly necessary. TMS null results are rarely published, which could partly be because null results are sometimes considered less exciting, unfortunately and unfairly, but could also be because of a traditional reluctance to interpret null findings; only because they are 'null findings'. We will first contrast TMS with other cognitive neuroscience methods, particularly functional magnetic resonance imaging (fMRI). We will then outline what we believe are common, if sometimes implicit, arguments against the interpretability of TMS null results, and consequently counter with reasons and conditions that do allow, after all, an interpretation of TMS null results. Such interpretation can be very informative and significantly heighten the potential of TMS research. To look ahead, we conclude that TMS null results can be meaningful, and often relevant enough to make public.

\section{Null results in functional neuroimaging}

Cognitive neuroscience assumes the basic premise that all manifestations of behavior and cognition arise from the brain. The goal of cognitive neuroscience is thus to establish and quantify the relations between brain processing and human faculties. The prime research paradigm in this endeavor seems to involve functional imaging. Electroencephalography (EEG), magnetoencephalography (MEG), positron emission tomography (PET), and functional magnetic resonance imaging (fMRI), are all functional imaging methods. Experiments using these different methods boast different spatiotemporal resolution profiles, making the possible applications and research questions versatile and method-dependent. But importantly, the underlying principle is always the same: to establish a correlation between brain activity on the one hand, and behavior (or cognition) on the other hand. While the blossoming field now hosts a growing and advanced set of analysis tools, continuously expanding the range of experimental questions that can be addressed, at the basis still lies the concept of 'human brain mapping'. Some regions of the brain are involved in cognitive process A, while other regions of the brain are involved in cognitive process $\mathrm{B}$. The goal is to find which regions do what (and increasingly, how). 


\section{An fMRI example}

Taking fMRI as an example, a typical experiment might involve two tasks. Task A is a visual angle discrimination task (henceforth referred to as "angle judgment"). Participants are shown images of analogue clocks and are asked to make a decision on the angle between the two clock-hands. Task B is a color discrimination task (henceforth referred to as "color judgment"). Participants are shown the same images, but are asked to make a decision on the color of the clock-hands. The MRI scanner measures metabolic activity throughout the brain (as in de Graaf et al., 2010; Sack et al., 2002a). The activity measured during task A (angle judgment) is then contrasted to the activity measured during task B (color judgment). Comparing throughout the brain where activity is stronger in one task than another, bilateral parietal cortices have been shown to be specifically active during angle judgment (Sack et al., 2007).

It becomes clear which role 'null results' play. At the heart of human brain mapping is the idea that some regions are involved in a particular task A, but that others are not. In part this remains implicit; a report on the neural correlates of angle judgments states that posterior parietal cortex is involved in angle judgments. It does not explicitly state that this temporal, this frontal, and that thalamic region are not involved. The lack (or reduction) of activation in these brain regions essentially constitutes null results. On reflection, they are integral to the positive finding: they allow us to believe that specifically that region is involved. A similar role is played by the activity in that same region but at other time points throughout the functional scan: the increased activity in a relevant trial is meaningful, because it is contrasted with the not-increased activity before and after. Again a similar role is played by the activity in that same region during the control task (color judgment). Parietal cortex might have been relevant for any kind of judgment, or any kind of task. The comparison with (lack of) activity during color judgment affords the most meaningful conclusion. All these 'null results', i.e. lower levels of BOLD response, are integral to the positive finding for parietal cortex during angle judgments. As this basic logic seems identical for most other human brain mapping experiments, the enterprise seems to only make sense if the results on regions shown to be active are taken as seriously as the results on regions not shown to be active, and as seriously as results of non-activation in the same regions but during other tasks.

\section{The added value of TMS}

In contrast to brain imaging, TMS can be classified as 'brain interference'. Neuroimaging is a correlational endeavor: regional activation in fMRI will indicate that the region was doing 'something' in relation to the task. However, this activity might have been crucial to task performance, or it might not have been. For example, considering the brain's known redundancy, maybe the activations reflect reserve 
capacity. Maybe the activations reflect post hoc analysis of the answer just provided. With repeated task performance, or practice, activations can decrease (Goodyear and Douglas, 2009) - possibly consolidating around the nodes that are actually necessary for the task. There is no way to know whether a particular brain region could be 'missed' without any noticeable effects on behavior. Since cognitive neuroscience is interested in understanding how brain relates to behavior, that leaves an important question unanswered. One way to address this issue is to use TMS.

With TMS, the region of interest can be stimulated; which means we can for example manipulate the excitability, and thereby amount of activity, in that region as an independent variable. We can then evaluate whether that particular region needs to be undisturbed for unimpaired task performance. This is the idea behind the 'virtual lesion' approach (Pascual-Leone et al., 2000). Alternatively, TMS can facilitate, rather than inhibit, processing in an area by enhancing excitability (Robertson et al., 2003). The direction of effects, inhibitory or excitatory, and duration and timing of effects, longerlasting or transient, depends on the TMS protocol used (Robertson et al., 2003; Sandrini et al., 2010). But whichever effect is obtained, if TMS over a region induces behavioral changes then undisturbed activity in that region is functionally relevant for that behavior. This reasoning leaves us with an open question: is the region of interest functionally relevant for the task, or is it not? If we pose the question like this, we will have to be ready to receive a positive and a negative answer. If we cannot accept a negative answer, we are cutting ourselves short.

\section{Null results in TMS}

TMS has diverse, adaptable parameters and applications (Pascual-Leone et al., 2000; Sack, 2006; Sandrini et al., 2010). A brain region can momentarily be stimulated or disturbed, or reversibly be deactivated for variable amounts of time. Parameters to 'tweak' are pulse shape, numbers of pulses, frequency of pulses, the use of bursts of pulses, numbers of pulses in bursts, inter-burst intervals, stimulation intensity. It is moreover possible that TMS pulses have different effects at different times in relation to a cognitive event (de Graaf et al., 2009), in different states of the cognitive system (Sack et al., 2007), and even in different participants (Maeda et al., 2002). In the "infinite parameter space" of TMS, there are more possible protocols than published TMS studies. But, not all TMS stimulations have neural effects (e.g. stimulation with too low intensity), and moreover not all TMS protocols with resulting brain activity changes have behavioral effects. The problem is, with TMS alone one cannot see into the black box that is the brain to establish what neural effect was actually achieved. Instead, one must rely on behavioral measures. These can include direct motor or perceptual effects ('behavioral markers' of TMS), or increased or decreased reaction times or performance accuracies in cognitive tasks. When TMS effects on these behaviors are obtained, they can be controlled for in several ways which all have their advantages and disadvantages (see Table 1). Analogous to null results in functional imaging, we suggest that the null results in TMS, as obtained in all these control conditions, are in fact crucial to the design and interpretation of TMS research. 
A significant TMS result tells us something fundamental (that a brain region is functionally relevant for a task), and can usually be published in appropriate journals. But what if TMS over region X has no effect on task A performance? It seems that we do not conclude so quickly that region $\mathrm{X}$ is not involved in task A. After all, maybe we needed different parameters, maybe we did not manage to target the region we wanted to stimulate, maybe the stimulation was not strong enough. One does not easily consider such null results very meaningful. But actually, we should consider one important point: TMS null results suddenly are interpreted as very meaningful when they serve as control data.

If task B serves as a control task, the null results on task B are interpreted as a lack of effect, nicely contrasting the positive finding in experimental task A. As in functional imaging, the experimental task findings are interpreted in contrast to the control findings. These control null results are thereby integral to the TMS research paradigm. But going forward from this: if task B was in fact the experimental task, should then the null results be cast aside? We suggest not.

Currently, there are hardly any TMS studies that have been published focusing on null results in the experimental condition of interest (although of course exceptions exist that prove the rule, e.g. Rossi et al., 2006). One step further than publishing 'failure to obtain positive findings', the stronger conclusion that region $\mathrm{X}$ is not important for task A based on null results seems even more rare. Then going one step further by actually performing a TMS study that sets out to show that a brain region is not functionally relevant, seems nearly inconceivable. That may be considered unfortunate, since it strongly reduces the number of research questions addressable by TMS.

\section{A conceptual dichotomy of meaningfulness in TMS results}

There thus seems to be an implicit dichotomy in the conceptual space of TMS findings. A dividing line separates significant from non-significant TMS results in the experimental condition: the significant results mean something, the non-significant results do not. Except, we have pointed out that this is actually not true, considering our interpretation of findings in control conditions. Moreover, TMS methodology has evolved since its conception. (f)MRI-guided TMS, with stereotactic frameless navigation, makes it possible to precisely target brain regions defined by either anatomical or functional landmarks (see below). Extensive explorations of TMS protocols inform us about robust and successful protocols, and durations of TMS effects. Combining TMS with methods of functional imaging, both in separate sessions as well as within the same experimental session, has identified the nature and spatial layout of the neural effects induced by certain TMS protocols over specific brain regions (Bestmann et al., 2005; Massimini et al., 2005; Ruff et al., 2006; Sack et al., 2007). We propose that these developments, implemented in well-designed experiments, now make it possible to conclude something, rather than nothing, from null results in TMS. 
In what follows, we aim to outline the main arguments researchers may have implicitly or explicitly, against the acceptance of null results as 'meaningful'. Subsequently, we counter these, ending with concrete recommendations for the design of TMS experiments.

\section{Arguments for and against interpretation of TMS null results}

\section{Arguments against null result interpretation}

In our view, there are three main arguments why researchers should not accept TMS null results as meaningful: the localization argument, the neural efficacy argument, and the power argument.

The localization argument is straightforward: perhaps the TMS coil was not properly positioned and the targeted brain region $\mathrm{X}$ was therefore not stimulated. We found that a very precise targeting of human motion area MT, on the basis of individual fMRI-based localizers and neuronavigation (see below) leads to behavioral effects on a motion discrimination task (Sack et al., 2006), whereas a coil displacement in the range of millimeters could abolish the effects. This shows that TMS research can be very sensitive to coil placement. Indeed, many more subjects are necessary to obtain significant TMS results when using, for instance, EEG 10/20 based coil positioning, as compared to individual fMRI-based neuronavigation (Sack et al., 2009a). Thus, it could be argued that a TMS null result in the experimental condition could always be due to 'missing the brain target'.

Second, the neural efficacy argument: we cannot be sure that the expected neural effects occurred, even if we targeted the right brain region. In other words, perhaps the targeted region X was not properly stimulated because of the selected TMS protocol. This makes intuitive sense, because in the "infinite parameter space" a different TMS protocol might have led to significant results, maybe TMS was per se not capable of eliciting any significant neural activity changes in that brain region either because the brain area was too distant from the surface of the coil or because it had too high an excitability threshold. This could mean that the intensity was too low, that the ongoing neural process was too robust to be significantly affected by TMS, or that the parameters were ill-chosen. In summary, maybe we did not successfully stimulate the brain as planned.

The power argument is very old, but particularly problematic for TMS research: perhaps testing more participants would have yielded a significant result. Since TMS is a brain interference method, it is both ethically and practically unacceptable to test hundreds, or sometimes even tens, of participants. Therefore, in many TMS studies the number of subjects hovers between 10 and 20, although a considerable amount of convincing and high-impact TMS studies involved fewer. 


\section{Counterarguments}

The first argument was the localization argument. In early TMS studies, anatomical landmarks on the skull guided coil placement. For instance, early studies targeting the human motion area hMT/V5 with a round TMS coil would be placed around $5 \mathrm{~cm}$ to the left or right of the Nasion-Inion line (e.g. Beckers and Homberg, 1992). Early visual cortex (often referred to as 'V1') generally was localized by targeting a few centimeters above the inion. In such visual studies (as well as motor studies), functional measurements could be used to provide some indication of the cortical region. For instance, for motor areas muscle twitches can be induced whereas for early visual cortex phosphenes can be induced in the appropriate visual field location (Barker et al., 1985; Hallett, 2007; Kammer et al., 2005). For the human motion area, a brief measurement could reveal if motion perception was at all affected by the TMS pulses, prior to the actual experiment. Using these clever solutions, very impressive work was done by early TMS pioneers. But what about silent cortical areas? What if no behavioral or perceptual marker is present to validate the coil position?

One way to be surer about which brain regions were stimulated in an experiment is the post hoc localization of 'likely' targeted cortical regions. This involves coregistration of coil placement with MRI images, for instance by 'highlighting' the stimulated location on the skull with a capsule that shows up clearly in an MRI scan. This yields some information about the consistency of TMS coil placement across subjects, and within a certain range provides an indication of the anatomical region that was (probably) stimulated.

However, today it is possible to implement real-time tracking of relative positions of TMS coil and participant anatomy using neuronavigation technology. Using reconstructed head and hemisphere meshes from MRI images, the researcher can see exactly where he/she stimulates, as the pulses are administered. This allows precise anatomical targeting (e.g. Schuhmann et al., 2009), or also functional targeting (e.g. de Graaf et al., 2009). TMS studies remain useful without imaging (Sack, 2010). But if functional imaging data are available, it is but a small step to overlay functional imaging results from previous fMRI sessions (e.g. using the same cognitive task A now being studied with TMS), allowing the researcher to online target that particular participant's activation 'hotspot'. A null result on a brain region $\mathrm{X}$ thus targeted would be more meaningful. The success of the coregistration procedure could easily be tested by navigating to for example the hand area of the motor cortex and eliciting muscle twitches. If one is thus sure of the coregistration, and has functional hotspots based on previous neuroimaging, but still finds no effects from TMS, this null result seems quite informative.

The power argument is a common problem. If no TMS effects are found, it could simply be that more trials and/or more participants would have led to significant results. This is true. But it is fundamentally true, always true, and true for all methods and all fields of research. It is at the same time an epistemological and a statistical problem, not a TMS problem. In fact, today we have an expanded knowledge base on 
what effect sizes can be expected in TMS research, on which brain regions and in which tasks. For instance, in TMS masking (Kammer, 2007a,b; Sack et al., 2009b), visual perception can be abolished completely, while in higher cognitive tasks (such as angle judgments: (Sack et al., 2002a,b), reaction times might be increased by a few percent. Our primary response to the power argument is therefore to look at effect sizes. If no TMS effect occurs at all, or an exceedingly small effect is in the 'wrong' direction, the power argument becomes less credible. If nothing is observed in ten participants, it is bad practice to test another ninety, just in case it was a power problem. We propose that, realistically and pragmatically, a power problem should mainly be considered if an effect occurs in the dataset, but it is simply not strong enough to reach significance. Looking at the 'effect sizes' of dependent variable fluctuations in control conditions, where no effects should occur, might additionally be useful in assessing the credibility of a power problem. If the effect size is in line with random fluctuations around the mean in control conditions, the power problem is less credible.

Lastly, the neural efficacy argument states, concisely, that in a TMS-only experiment, you cannot know if your TMS had the desired neural effects. Perhaps the TMS was not strong enough to actually disturb the redundant organizational processing in that particular region. Or perhaps TMS did disrupt processing in the targeted region to a sufficient degree, but other regions were able to compensate for the 'virtually lesioned' brain area (Sack et al., 2005). Today, there are ways to address these issues.

Firstly, researchers could look into the brain, opening up the black box. Simultaneous TMS-EEG and TMS-fMRI are possible and documented (Sack et al., 2007; Sandrini et al., 2010; Thut and Pascual-Leone, 2010), with very revealing results. An analogy with lesion studies in patients is useful. Null results in lesion studies are generally acceptable, since if a brain region is identifiably gone, and a cognitive process is not affected, that brain region cannot be necessary for that process. That logic is so common that even case studies are accepted and published. An MRI scan can show which exact region was lesioned (thus; looking into the brain which exact region was affected). Similarly, in TMS, if a simultaneous (or immediately subsequent) brain scan can show that the TMS protocol did affect the brain, and in which way, both positive and negative TMS results can become very meaningful. This method is useful particularly if compensatory processes are hypothesized in the brain; they might thus be revealed and visualized.

Secondly, a more fundamental efficacy check is possible without having to resort to imaging methods. This neural efficacy check requires a behavioral effect of the TMS protocol on the brain region of interest in the subject sample of the actual experiment. As mentioned above, an independent TMS localization procedure might be performed. For instance, if the region of interest (hypothetically involved in task A) is frontal eye field (FEF), an independent task might be tested. It is known that FEF is responsible for saccades and that TMS can affect saccades (Leff et al., 2001; Ro et al., 2002). Thus, if in subject S, TMS over target region $X$ (supposedly FEF) leads to affected saccades, there is at once direct evidence that the used TMS protocol has neural effects in region $\mathrm{X}$, and that the region $\mathrm{X}$ is likely to be FEF - making later null results of TMS on task A more meaningful. For motor cortex and visual cortex, there are the aforementioned motor-evoked potentials and phosphenes respectively. But solutions are 
developed for 'silent' association areas as well, which are involved in more cognitive, psychologically interesting processes. In some cases, 'hunting procedures' can be applied for association areas (e.g. Oliver et al., 2009). The basic logic here is that known and robust TMS effects for particular areas can be used to find these brain areas, after which these same areas in the same subjects can be stimulated (ideally with the same TMS parameters) in an experimental task of interest for which the functional relevance of that area is an open question.

We propose that a related neural efficacy check may be possible in silent cortical areas. As mentioned, if stimulation of brain region $\mathrm{X}$ has effects on task $\mathrm{A}$, but not on task $\mathrm{B}$, we generally conclude that region $\mathrm{X}$ is responsible for task $\mathrm{A}$. But implicitly or explicitly, we thereby also conclude that region $\mathrm{X}$ is not responsible for task B. This is definitely the case, even if implicitly, if no other controls are included in the study. In this way, null results have been 'interpreted' as meaningful many times before in TMS, although not focused on. To deal with the neural efficacy argument, we now simply suggest that one might turn this logic around. If one wants to show that a region is not functionally relevant for task A, then one might endeavor to find a task B that is closely related, uses the same stimuli, and is at the same level of difficulty, but on which the applied TMS protocol does have an effect. (In online TMS paradigms, where stimulation is temporally specific, the timing of the magnetic stimulation should additionally be considered.) In the sketched scenario, if TMS has significant effects on task $\mathrm{B}$, but not on the experimental task $\mathrm{A}$, the positive finding on control task $\mathrm{B}$ becomes the control condition that validates the null result on experimental task $\mathrm{A}$. Since the mirror of this procedure is done regularly in TMS experiments that focus on the positive findings, we do not see a problem with this. In fact, this is exactly what we did in the previous chapter, when we obtained positive TMS findings on the voluntary control task, but null results on the passive viewing task. We concluded that the stimulated region, responsible for voluntary control, was not also responsible for inducing switches in passive viewing. After all, we could be sure that our TMS protocol successfully inhibited the brain region of interest: a neural efficacy check.

Using TMS to try to explicitly demonstrate that a particular region is not functionally relevant is one advantage of taking null results seriously, and a road not often travelled. A more general advantage is the potential increase in efficiency across TMS labs. If not only successful studies were made public, but also unsuccessful studies where no significant effects were found, TMS research as a whole would benefit. We will now turn to this pragmatic consideration.

\section{Pragmatic considerations of TMS null results}

Taking all these considerations together, a lack of behavioral effects for a region of interest in a sophisticated TMS experiment would become, in our opinion, rather interesting. But even without a neural efficacy check, to take a pragmatic viewpoint, we can now be reasonably sure about a number of things. The infinite parameter space plaguing TMS experiments has in recent years been confined somewhat. In part through experience, in part through biophysical insights, it is now fairly established that certain 
TMS protocols simply do have replicable and predictable neural effects (excitatory or inhibitory). Moreover, still newer protocols such as theta-burst TMS (Huang et al., 2005) seem to have even more robust and lasting neural, and possibly behavioral, effects. Furthermore, newer paradigms are developed that use TMS in new ways, such as TMS-adaptation or TMS-priming, asking increasingly specific questions about the functional roles of brain regions (Cattaneo et al., 2008; Cattaneo and Silvanto, 2008; Gilaie-Dotan et al., 2010; Silvanto and Pascual- Leone, 2008). All in all, it is no longer realistic to doubt as a matter of principle that TMS could have inhibited or excited a region. We can begin to trust in communal knowledge that certain protocols with certain machine output intensities actually DO affect the brain in reliable ways. We suggest that, therefore, null results might also be added to the communal knowledge base.

In any research field, the current state of knowledge always yields ideas for further experiments. Therefore, different labs are often working on the same questions. With a growing TMS community and expanding TMS application venues, the overlap is likely to increase. To adopt a stance that works toward the furthering of knowledge: it seems to us desirable that different research groups do not repeat the same experiments in ignorance of each others' efforts. So, aside from all the suggested reasons to submit TMS null results to interpretation, simply the fact that certain established TMS protocols in certain tasks did not result in significant behavioral effects might reasonably be of interest to the community. This is of course a very pragmatic

\begin{tabular}{|c|c|c|c|c|}
\hline Control type & Description & Controls for... & Example & (Dis) advantages \\
\hline No TMS & $\begin{array}{l}\text { Trials or blocks without TMS. } \\
\text { Preferably these are } \\
\text { interleaved randomly with } \\
\text { TMS trials/blocks }\end{array}$ & $\begin{array}{l}\text { Provides a baseline, or } \\
\text { reference, for the TMS trials }\end{array}$ & $\begin{array}{l}\text { Angle judgment trials without } \\
\text { TMS }\end{array}$ & $\begin{array}{l}\text { Single-pulse TMS: sudden lack of } \\
\text { pulse might startle; repetitive } \\
\text { TMS: whole block without } \\
\text { concurrent TMS can have } \\
\text { fundamentally different effects on } \\
\text { non-specific factors such as arousal }\end{array}$ \\
\hline Sham TMS & $\begin{array}{l}\text { Trials or blocks with TMS from } \\
\text { a SHAM coil (which sounds and } \\
\text { looks genuine but releases } \\
\text { low- or no-intensity pulses) }\end{array}$ & $\begin{array}{l}\text { The non-specific disruptive } \\
\text { effects of TMS, such as auditory } \\
\text { and (to a degree) } \\
\text { somatosensory stimulation, as } \\
\text { well as levels of arousal and } \\
\text { vigilance }\end{array}$ & $\begin{array}{l}\text { Angle judgment trials with } \\
\text { sham TMS pulses }\end{array}$ & $\begin{array}{l}\text { Better than no TMS, does not } \\
\text { require giving pulses to a region } \\
\text { (or at a time) where effects are not } \\
\text { expected, which is ethically } \\
\text { preferable }\end{array}$ \\
\hline Control task & $\begin{array}{l}\text { A task, preferably on the same } \\
\text { stimuli, that requires a } \\
\text { different cognitive process for } \\
\text { which no TMS effects are } \\
\text { expected }\end{array}$ & $\begin{array}{l}\text { Specificity of the TMS effects } \\
\text { for the cognitive process of } \\
\text { interest }\end{array}$ & $\begin{array}{l}\text { Color judgment trials versus } \\
\text { angle judgment trials }\end{array}$ & $\begin{array}{l}\text { The subject is blind to which } \\
\text { conditions are experimental and } \\
\text { which are control }\end{array}$ \\
\hline Control site & $\begin{array}{l}\text { A brain region that is } \\
\text { stimulated, while the subject } \\
\text { performs the experimental } \\
\text { task of interest, where no TMS } \\
\text { effects are expected because } \\
\text { presumably it is not involved } \\
\text { in the cognitive process }\end{array}$ & $\begin{array}{l}\text { Specificity of the TMS effects } \\
\text { for the brain region stimulated } \\
\text { (and therefore controls also for } \\
\text { non-specific TMS effects } \\
\text { described under sham TMS) }\end{array}$ & $\begin{array}{l}\text { Vertex stimulation versus } \\
\text { parietal cortex stimulation }\end{array}$ & $\begin{array}{l}\text { The subject is blind to which } \\
\text { conditions are experimental and } \\
\text { which are control }\end{array}$ \\
\hline Control timing & $\begin{array}{l}\text { Particularly for single-pulse } \\
\text { TMS experiments, certain time } \\
\text { windows (with a particular } \\
\text { SOA) may yield TMS effects } \\
\text { whereas other time windows } \\
\text { do not. These become timing } \\
\text { control conditions }\end{array}$ & $\begin{array}{l}\text { Specificity of the TMS effects } \\
\text { for temporal windows }\end{array}$ & $\begin{array}{l}\text { In TMS masking of visual } \\
\text { stimuli TMS pulses } \\
\text { immediately ( }(10 \mathrm{~ms}) \text { after } \\
\text { stimulus onset }\end{array}$ & $\begin{array}{l}\text { The subject is blind to which } \\
\text { conditions are experimental and } \\
\text { which are control }\end{array}$ \\
\hline Effect duration & $\begin{array}{l}\text { Particularly for offline } \\
\text { repetitive TMS experiments } \\
\text { with lasting TMS effects: } \\
\text { measurements continue after } \\
\text { the TMS effects 'wear off the } \\
\text { later measurements can be } \\
\text { used as control measurements }\end{array}$ & $\begin{array}{l}\text { Provides convincing support } \\
\text { for the effects being neural TMS } \\
\text { effects (which are supposed to } \\
\text { 'wear off after a certain time) }\end{array}$ & $\begin{array}{l}\text { An inhibitory }(1 \mathrm{~Hz}) \mathrm{rTMS} \\
\text { protocol of } 10 \mathrm{~min} \text { with } \\
\text { behavioral measurements for } \\
\text { one hour afterwards (effects } \\
\text { should have definitely worn off } \\
\text { after, for example, } 30 \text { min) }\end{array}$ & $\begin{array}{l}\text { The subject is blind to which } \\
\text { conditions are experimental and } \\
\text { which are control, but a } \\
\text { disadvantage is that there is is no } \\
\text { way to counter order effects }\end{array}$ \\
\hline
\end{tabular}

Table 1. Controls in TMS 
argument, and not so much new. But on the other hand, new journals, particularly open source and digital-only journals, do for the first time openly advertise that studies can be published on methodological merit rather than significance of results. In other words; perhaps it is also becoming easier to publish data that are not statistically significant.

\section{Conclusion}

There should not be a taboo on TMS null results. By designing experiments along the lines suggested above; using neuronavigation, behavioral markers, neural efficacy checks, and implementing established TMS protocols with known neural effects, researchers can be rather confident in what they did and did not accomplish in terms of brain interference. Therefore, it has become possible to obtain meaningful TMS null results. This is important, because a TMS null result may be the only way to show, in healthy human subjects, that a brain region $\mathrm{X}$ is not functionally relevant for a task $\mathrm{A}$. 


\section{References}

Barker, A.T., Jalinous, R., Freeston, I.L., 1985. Non-invasive magnetic stimulation of human motor cortex. Lancet 1, 1106-1107.

Beckers, G., Homberg, V., 1992. Cerebral visual motion blindness: transitory akinetopsia induced by transcranial magnetic stimulation of human area V5. Proc. Biol. Sci. 249, 173-178.

Bestmann, S., Baudewig, J., Siebner, H.R., Rothwell, J.C., Frahm, J., 2005. BOLD MRI responses to repetitive TMS over human dorsal premotor cortex. Neuroimage 28, 22 29.

Cattaneo, Z., Rota, F., Vecchi, T., Silvanto, J., 2008. Using state-dependency of transcranial magnetic stimulation (TMS) to investigate letter selectivity in the left posterior parietal cortex: a comparison of TMS-priming and TMS-adaptation paradigms. Eur. J. Neurosci. 28, 1924-1929.

Cattaneo, Z., Silvanto, J., 2008. Time course of the state-dependent effect of transcranial magnetic stimulation in the TMS-adaptation paradigm. Neurosci. Lett. 443, 82-85.

de Graaf, T.A., Jacobs, C., Roebroeck, A., Sack, A.T., 2009. Comparing FMRI effective connectivity and TMS chronometry: complementary accounts of causality in the visuospatial judgment network. PLoS ONE 4 (12), e8307.

de Graaf, T.A., Roebroeck, A., Goebel, R., Sack, A.T., 2010. Brain network dynamics underlying visuospatial judgment: an fMRI connectivity study. J. Cogn. Neurosci., 22.

Gilaie-Dotan, S., Silvanto, J., Schwarzkopf, D.S., Rees, G., 2010. Investigating representations of facial identity in human ventral visual cortex with transcranial magnetic stimulation. Front. Hum. Neurosci. 4, 50.

Goodyear, B.G., Douglas, E.A., 2009. Decreasing task-related brain activity over repeated functional MRI scans and sessions with no change in performance: implications for serial investigations. Exp. Brain Res. 192, 231-239.

Hallett, M., 2007. Transcranial magnetic stimulation: a primer. Neuron 55, 187-199. Huang, Y.Z., Edwards, M.J., Rounis, E., Bhatia, K.P., Rothwell, J.C., 2005. Theta burst stimulation of the human motor cortex. Neuron 45, 201-206.

Kammer, T., 2007a. Masking visual stimuli by transcranial magnetic stimulation.Psychol. Res. 71, 659-666.

Kammer, T., 2007b. Visual masking by transcranial magnetic stimulation in the first 80 milliseconds. Adv. Cogn. Psychol. 3, 177-179.

Kammer, T., Puls, K., Erb, M., Grodd, W., 2005. Transcranial magnetic stimulationin the visual system. II. Characterization of induced phosphenes and scotomas.Exp. Brain Res. 160, 129-140.

Leff, A.P., Scott, S.K., Rothwell, J.C., Wise, R.J., 2001. The planning and guiding of reading saccades: a repetitive transcranial magnetic stimulation study. Cereb. Cortex 11, 918-923.

Maeda, F., Gangitano, M., Thall, M., Pascual-Leone, A., 2002. Inter- and intraindividual variability of paired-pulse curves with transcranial magnetic

stimulation (TMS). Clin. Neurophysiol. 113, 376-382.

Massimini, M., Ferrarelli, F., Huber, R., Esser, S.K., Singh, H., Tononi, G., 2005. Break-down of cortical effective connectivity during sleep. Science 309, 2228-2232. 
Oliver, R., Bjoertomt, O., Driver, J., Greenwood, R., Rothwell, J., 2009. Novel 'hunting' method using transcranial magnetic stimulation over parietal cortex disrupts visuospatial sensitivity in relation to motor thresholds. Neuropsychologia 47,31523161.

Pascual-Leone, A., Walsh, V., Rothwell, J., 2000. Transcranial magnetic stimulation in cognitive neuroscience - virtual lesion, chronometry, and functional connectivity. Curr. Opin. Neurobiol. 10, 232-237.

Ro, T., Farne, A., Chang, E., 2002. Locating the human frontal eye fields with transcranial magnetic stimulation. J. Clin. Exp. Neuropsychol. 24, 930-940. Robertson, E.M., Theoret, H., Pascual-Leone, A., 2003. Studies in cognition: the prob- lems solved and created by transcranial magnetic stimulation. J. Cogn. Neurosci. 15, 948-960.

Rossi, S., Pasqualetti, P., Zito, G., Vecchio, F., Cappa, S.F., Miniussi, C., Babiloni, C., Rossini, P.M., 2006. Prefrontal and parietal cortex in human episodic memory: an interference study by repetitive transcranial magnetic stimulation. Eur. J. Neurosci. 23, 793-800.

Ruff, C.C., Blankenburg, F., Bjoertomt, O., Bestmann, S., Freeman, E., Haynes, J.D., Rees, G., Josephs, O., Deichmann, R., Driver, J., 2006. Concurrent TMS-fMRI and psychophysics reveal frontal influences on human retinotopic visual cortex. Curr. Biol. $16,1479-1488$.

Sack, A.T., 2006. Transcranial magnetic stimulation, causal structure-function mapping and networks of functional relevance. Curr. Opin. Neurobiol. 16, 593-599.

Sack, A.T., 2010. Does TMS need functional imaging? Cortex 46, 131-133.

Sack, A.T., Camprodon, J.A., Pascual-Leone, A., Goebel, R., 2005. The dynamics of interhemispheric compensatory processes in mental imagery. Science 308, 702-704.

Sack, A.T., Cohen Kadosh, R., Schuhmann, T., Moerel, M., Walsh, V., Goebel, R., 2009a. Optimizing functional accuracy of TMS in cognitive studies: a comparison of methods. J. Cogn. Neurosci. 21, 207-221.

Sack, A.T., Hubl, D., Prvulovic, D., Formisano, E., Jandl, M., Zanella, F.E., Maurer, K., Goebel, R., Dierks, T., Linden, D.E., 2002a. The experimental combination of rTMS and fMRI reveals the functional relevance of parietal cortex for visuospatial functions. Brain Res. Cogn. Brain Res. 13, 85-93.

Sack, A.T., Kohler, A., Bestmann, S., Linden, D.E., Dechent, P., Goebel, R., Baudewig, J., 2007. Imaging the brain activity changes underlying impaired visuospatial judgments: simultaneous FMRI, TMS, and behavioral studies. Cereb. Cortex 17, 28412852.

Sack, A.T., Kohler, A., Linden, D.E., Goebel, R., Muckli, L., 2006. The temporal charac- teristics of motion processing in hMT/V5+: combining fMRI and neuronavigated TMS. Neuroimage 29, 1326-1335.

Sack, A.T., Sperling, J.M., Prvulovic, D., Formisano, E., Goebel, R., Di Salle, F., Dierks, T., Linden, D.E., 2002b. Tracking the mind's image in the brain II: transcranial mag- netic stimulation reveals parietal asymmetry in visuospatial imagery. Neuron 35 , 195-204.

Sack, A.T., van der Mark, S., Schuhmann, T., Schwarzbach, J., Goebel, R., 2009b. Symbolic action priming relies on intact neural transmission along the retino-geniculostriate pathway. Neuroimage 44, 284-293. 
Sandrini, M., Umilta, C., Rusconi, E., 2010. The use of transcranial magnetic stimulation in cognitive neuroscience: a new synthesis of methodological issues. Neurosci. Biobehav. Rev. 35, 516-536.

Schuhmann, T., Schiller, N.O., Goebel, R., Sack, A.T., 2009. The temporal characteristics of functional activation in Broca's area during overt picture naming. Cortex 45, 1111-1116.

Silvanto, J., Pascual-Leone, A., 2008. State-dependency of transcranial magnetic stimulation. Brain Topogr. 21, 1-10.

Thut, G., Pascual-Leone, A., 2010. Integrating TMS with EEG: how and what for? Brain Topogr. 22, 215-218. 



\title{
III.3
}

\section{Alpha-band Rhythms in Visual Task}

\author{
Performance: phase-locking by
}

rhythmic sensory stimulation

Related publication(s):

de Graaf, TA, Gross, J*, Paterson, G*, Rusch, T*, Sack, AT, Thut, G* (2013). Alphaband rhythms in visual task performance: phase-locking by rhythmic sensory stimulation. PloS ONE. (accepted)

* Joachim Gross, Gavin Paterson, Gregor Thut

1) Centre of Cognitive NeuroImaging, Institute of Neuroscience and Psychology, University of Glasgow, United Kingdom

* Tessa Rusch

1) Department of Psychology, Ludwig Maximilians University Munich, Germany 


\begin{abstract}
A brief event in one sensory modality can lead to rhythmicity in subsequent stimulus-detection in the same or another modality. This rhythmicity in performance measures is time-locked to the initial event, cycles at typical frequencies of brain-rhythms and may result from phase-resetting of ongoing brain oscillations. We here studied whether rhythmic sensory stimulation at frequencies of sensory brain areas may reveal implication of these brain oscillations in behaviour. If so, rhythmic sensory stimulation tuned to frequencies of sensory brain-rhythms 1) should have specific behavioural consequences relative to stimulation at flanker frequencies, and 2) should result in time-locked oscillations in performance measures, that 3 ) relate to brain oscillations over sensory areas. We here considered this hypothesized impact of rhythmic sensory events for the visual system and one of its best-characterized rhythms, the alpha-oscillations $(8-12 \mathrm{~Hz})$. We presented rhythmic visual cues at frequencies ranging from $3.9-17 \mathrm{~Hz}$ and tested their impact on subsequent visual target detection at cued/uncued positions. First, we found a frequency-specific breakdown of cueing benefits for $10 \mathrm{~Hz}$-stimulation (in the alpha-band) in comparison to stimulation at flanker frequencies (outside this band). Second, 10Hz-stimulation led to an alpha-oscillation in visual task performance post-cueing but only at cued, not uncued positions. Interestingly, while $10 \mathrm{~Hz}$ cues were presented in the hemifield opposite to the final target (rhythmic motion cueing), cyclic patterns in perception were present, suggesting attention-related phase-reset in the alpha band. Third, this sinusoidal pattern of visual performance correlated in frequency across individuals with resting encephalographic alpha-oscillations over occipital areas. The most parsimonious explanation for these three findings is entrainment (phase-locking) of ongoing perceptually relevant brain oscillations by rhythmic sensory events. This would suggest that rhythmic stimulation can be used to reveal influences of brain-rhythms on task performance to study their functional roles, here in line with occipital alpha-oscillations underlying periodicity in visual performance.
\end{abstract}




\section{Introduction}

Rhythms are ubiquitous in biological systems. From the sniffing of rodents (Schroeder, Wilson et al. 2010) to the structure of the human attention system (VanRullen, Carlson et al. 2007), temporal regularity is a fundamental property for organisms. While we have been aware of such periodicity in the brain (Buzsaki 2006), a number of recent studies has emphasized that rhythmicity is also apparent in behavior.

A single event can 'reverberate' in perceptual systems, leading to an oscillatory pattern in visual task performance time-locked to the event. One sound, when predictive of upcoming visual events, can lead to a cyclic pattern in visual target detection (Fiebelkorn, Foxe et al. 2011) and visual cortex reactivity (Romei, Gross et al. 2012). A single visual flash, presented in one hemifield to capture attention, results in cyclic patterns of visual detection behavior in this hemifield and opposing (antiphase) detection behaviour in the opposite hemifield (Landau and Fries 2012). Multiple events, if in a stable rhythm, have similar effects. Rhythmic trains of events generally benefit processing of subsequent stimuli, if these are in phase with the preceding train (Jones, Moynihan et al. 2002; Doherty, Rao et al. 2005; Mathewson, Fabiani et al. 2010; Rohenkohl, Coull et al. 2011). Continuous streams of multiple but non-periodic (random) events can be associated with flicker perception at a specific frequency (see (VanRullen and Macdonald 2012) for 'perceptual echoes'). Such findings suggest a fundamental role for periodicity in perception and attention.

At the same time, oscillations in the brain have been linked to perception, attention and exploratory behaviour. The phase of ongoing brain oscillations predicts somatosensory detection performance (Monto, Palva et al. 2008), visual detection performance (Busch, Dubois et al. 2009; Mathewson, Gratton et al. 2009) and saccadic latency (Hamm, Dyckman et al. 2010; Drewes and VanRullen 2011). Sensory events can align the phase of oscillatory brain activity, a process linked to and amenable to attention (Lakatos, Karmos et al. 2008). The effects of phase of oscillatory brain activity on perception can depend on the power of these oscillations (Mathewson, Gratton et al. 2009), and power and phase of oscillatory brain activity can be modulated by rhythmic sensory stimulation (Galambos, Makeig et al. 1981; Herrmann 2001; Shang, Dan et al. 2011; Thut, Schyns et al. 2011). Collectively, these findings raise the hypothesis that periodicity in perception is closely linked to oscillations in the brain (Vanrullen and Dubois 2011) and that sensory stimulation may be used to study the link between perception and oscillations via phase-reset of the two.

One prominent rhythm of the visual system is the occipito-parietal alphaoscillation $(8-12 \mathrm{~Hz})$. Its power is modulated by visual attention deployment in space (Foxe and Snyder 2011) as well as time (Rohenkohl and Nobre 2011) and shows an inverse relationship to visual performance (Thut, Nietzel et al. 2006; Hanslmayr, Aslan et al. 2007; Romei, Brodbeck et al. 2008; van Dijk, Schoffelen et al. 2008; Romei, Gross et al. 2010). Here, we further consider the link between the occipital alpharhythm in the brain and perception and attention, by using rhythmic sensory stimulation and probing its effects on subsequent visual target detection. Rhythmic $10 \mathrm{~Hz}$ visual stimulation has been shown to enhance occipital-parietal alpha power (Herrmann 2001; 
Ding, Sperling et al. 2006; Shang, Dan et al. 2011). This raises the question whether this is due to phase-locking of naturally occurring alpha oscillations that may causally modulate visual task performance. If so, this should have a number of testable consequences on visual performance measures. First, rhythmic stimulus trains at alpha frequency $(10 \mathrm{~Hz})$ should disproportionally interfere with visual/attentional performance, relative to stimulation at flanker frequencies outside the alpha-band (alpha-specific effects) supporting the view that alpha-power and perception are inversely related and in line with its proposed inhibitory role (Klimesch, Sauseng et al. 2007). Second, after rhythmic alpha-stimulation, visual task performance should

A

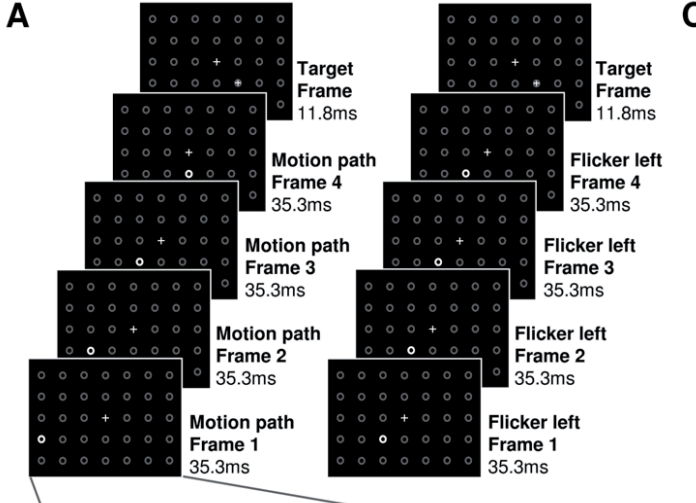

B

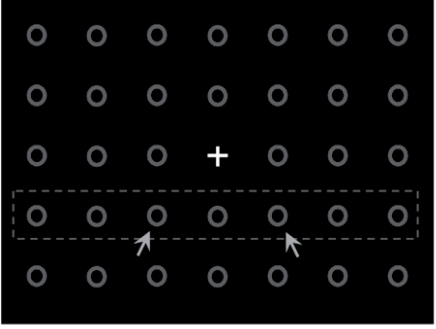

D
C

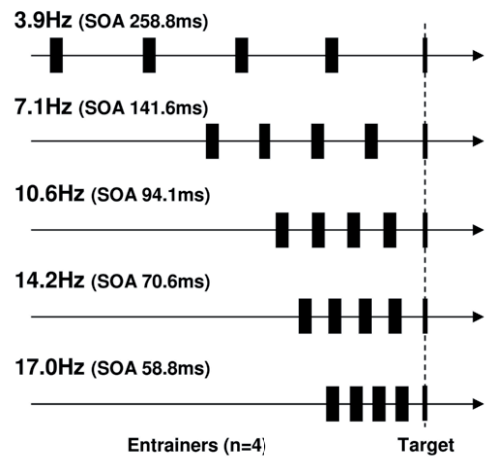

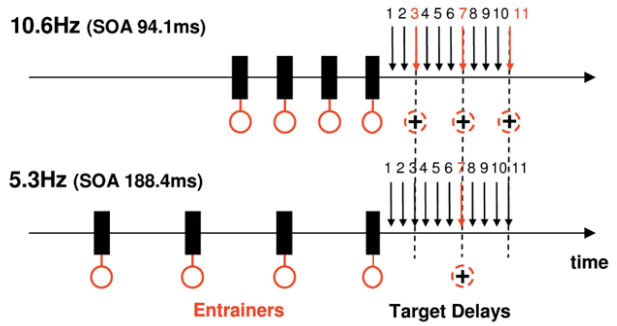

Figure 1: Stimuli and Design.

A. A 5x7 array of annuli and a fixation cross were presented at all times. Rhythmic cueing consisted of the briefly $(35.3 \mathrm{~ms})$ brightening ('flashing') of annuli in a predictable sequence. In 'motion entrainment' (left), 4 annuli would flash along a spatial path, starting either with the left-most (example) or right-most annulus, and ending with the central annulus below the fixation cross. A visual target was then presented in the centre of the next annulus of the motion path (cued/congruent location) or in the annulus that flashed before (incongruent location). In 'flicker entrainment' (right), the same annulus would flash 4 times. The flashing annulus could be at the left (example) or right target locations. Depending on whether the target location coincided with the cued location, cueing was either congruent or incongruent. For both forms of entrainment, targets appeared at congruent and incongruent positions with equal likelihood, thus cueing was spatially non-predictive. B. Enlarged view of the stimulus array, indicating for illustration purposes by rectangle the row of annuli that were involved in motion/flicker cueing, and by arrows the two possible target locations. C. Design of Experiment 1 testing five cueing frequencies. Visual targets were always 'in-phase' with the cue-train. D. Design of Experiment 2 testing two cueing frequencies and 11 stimulus onset asynchronies (from last cue to visual target). These covered three cycles at alpha frequency. 
oscillate over time with alpha-frequency (periodicity in perception) if perception depends on (alpha) phase. Third, these behavioral alpha oscillations should correlate in frequency with naturally occurring alpha oscillations in the brain (linking the above effects in behavior to brain rhythms), which would speak in favor of a causal role of these oscillations in perception.

In the current study, we tested these predictions in two experiments by examining the spatial and temporal (cyclic) profiles of visual performance after rhythmic visual stimulation at different frequencies (3.9 vs. 7.1 vs. 10.6 vs. 14.2 vs. $17 \mathrm{~Hz}$, one frequency in the alpha-band $=10.6 \mathrm{~Hz}$ and two flanker frequencies below and two above this band $=3.9 / 7.1 \mathrm{~Hz}$ and $14.2 / 17 \mathrm{~Hz}$ ) and correlating individual periodicity in visual performance with individual resting alpha-oscillations in the brain.

\section{Materials and Methods}

\section{Participants}

For experiment 1 , a total of 22 participants volunteered. Two were authors of this paper (T.R., T.A.G.), 20 were students at Glasgow University, receiving course credits for participation. One participant was excluded due to outlier performance (reaction times $>2.5$ standard deviations (SD) above group average, hit rate $>2 \mathrm{SD}$ below group average), leaving a total of 21 participants ( 5 male, $22 \pm 3$ yrs old, 4 left-handed). For experiment 2, 20 participants volunteered. Two were authors of this paper (T.R., T.A.G.). 18 were students at Glasgow University, compensated with course credits. Two participants were excluded on the basis of outlier performance (reaction times $>2.5 \mathrm{SD}$ above group average, or hit rate $>2.5 \mathrm{SD}$ below group average). In total 18 subjects were included in the analysis (10 male, $23 \pm 4 \mathrm{yrs}, 3$ left-handed). All subjects had normal or corrected-to-normal vision. This work was approved by the ethics committee of the institution where measurements took place (Centre of Cognitive NeuroImaging, Institute of Neuroscience and Psychology, University of Glasgow, United Kingdom). All subjects provided written informed consent.

\section{Rhythmic Stimulation Paradigms}

We implemented two rhythmic stimulation paradigms: a stationary as well as an apparent motion entrainment/cueing paradigm ('flicker' versus 'motion' cues). We call these paradigms interchangeably 'entrainment' or 'cueing' as the rhythmic stimulus train (the 'entrainers') cues for the upcoming visual target. Our design for 'flicker' entrainment (cueing) was inspired from Mathewson et al. (Mathewson, Fabiani et al. 2010), who showed that rhythmically presenting a visual annulus at one position benefits perception of an upcoming visual target at this position, when targets were presented in phase with the preceding train. Only a few rhythmic pre-target cues $(n=2-$ 8) sufficed to benefit perception. Our design for 'motion' entrainment (cueing) was inspired from Doherty et al. (Doherty, Rao et al. 2005) who showed that a visual disk rhythmically crossing the computer monitor benefits subsequent target perception if the 
target position and/or timing were predictable from the entrainment cues. In our modified versions of these paradigms, 'flicker' and 'motion' cueing were implemented as follows.

A matrix of $5 \times 7$ annuli and a central fixation cross were presented at all times on the screen (grey on black background, Fig 1A and B). We refer to these annuli as 'placeholders'. Entrainers consisted of placeholders briefly 'lighting up', or 'flashing'. During flicker entrainment, one placeholder would flash four times consecutively, before a visual target was presented (see Figure 1A, right for one example trial). Only placeholders of two positions could flash (diagonal to the lower right or lower left of the fixation cross, marked in Fig 1B for illustration purposes by arrows), and targets could appear either in the center of the placeholder that flashed, or in the opposite hemifield with 1:1 probability. Target position could thus be congruent or incongruent relative to cued position, but importantly, flicker cues were spatially non-predictive as to target positions. During motion entrainment, four placeholders of the row of circles below the fixation cross (marked in Fig 1B for illustration purposes by a rectangle) would flash in succession, either starting with the right-most circle and ending with the central circle directly underneath the fixation cross, or starting with the left-most circle and ending with the same central circle (see Figure 1A, left for one example trial). This was followed by a target presented in the adjacent placeholders, left or right from the last entrainer with 1:1 probability (i.e. in or out-of motion path, and at the same positions as the targets of the flicker condition, marked in Fig 1B). Thus, again target positions could be congruent or incongruent relative to the direction of motion cueing, but motion cues were spatially non-predictive as to target positions.

We foremost expected an advantage of visual task performance at cued versus uncued positions (in line with (Doherty, Rao et al. 2005; Mathewson, Fabiani et al. 2010)). Entrainers flashing at/towards the left or right position (flicker/motion cues) should benefit detection at cued (left or right) location. This is likely associated with changes to brain oscillations such as phase-alignment and/or power-modulations driven by attention (see Introduction). Note that our task discouraged endogenous cueing (by rendering target appearance at cued and uncued positions equally probable), and emphasized exogenous cueing to specific positions (by the spatiotemporal structure of the rhythmic stimulus trains). Irrespective of the mechanisms employed, we expected (1) this cueing benefit to be disproportionally affected by alpha-entrainment/cueing, (2) visual task performance to cycle at alpha-frequency, and (3) periodicity in perception to correlate with periodicity in brain rhythms, if brain oscillations in the alpha-band indeed play a role in perception.

\section{Experimental settings, stimulus parameters, and task}

Participants were seated $0.30 \mathrm{~m}$ in front of a CRT monitor (refresh rate $85 \mathrm{~Hz}$ ). Viewing distance was kept stable using a chin rest. In an initial training phase, participants were familiarized with the task, and target salience was individually adjusted to approximately $80 \%$ hit rate to avoid ceiling effects. The training phase consisted of a subset of trials but included all different conditions tested in the main experiment, and was repeated until stable performance was reached. 
The annuli of the placeholder matrix were $1.5 \mathrm{~cm}$ in diameter, spaced apart $5.3 \mathrm{~cm}$ horizontally and $5 \mathrm{~cm}$ vertically. For entrainment, annuli briefly changed (flashed) from grey to white for three frames $(35.3 \mathrm{~ms})$. Visual targets consisted of an ' $x$ ' or ' + ' and were always presented for the duration of one frame only $(11.8 \mathrm{~ms})$. Participants were asked to fixate the central fixation cross at all times. The task was to indicate by means of button presses whether a ' + ' or an ' $x$ ' sign (rotated ' + ') appeared on screen.

\section{Testing for alpha-specificity of effects (experiment 1)}

In experiment 1 , we evaluated the effects of congruency (cued vs. uncued position), and rhythmic cueing type (flicker vs. motion). To test for frequency-specificity, we implemented entrainment at five frequencies $(3.9 \mathrm{~Hz}, 7.1 \mathrm{~Hz}, 10.6 \mathrm{~Hz}, 14.2 \mathrm{~Hz}, 17 \mathrm{~Hz}$, Fig 1C). Note that for all conditions of Experiment 1 (motion vs. flicker, congruent vs. incongruent, 5 frequencies), visual targets followed 'in phase' with the entrainment, i.e, a regular interstimulus interval was used for presenting the four entrainers and the subsequent targets (Fig 1C) such that the onset of the visual target coincided with the onset of what would have been a fifth entrainer in the train. All conditions were presented in random order across trials in five runs. A total of 1200 trials were sampled, resulting in 60 trials per condition cell per participant.

\section{Testing for periodicity in visual performance measures (experiment 2)}

In experiment 2 only motion entrainment was implemented. Aside from testing for congruency effects (see above), we tested entrainment at two frequencies $(10.6 \mathrm{~Hz}$, $5.3 \mathrm{~Hz}$ ) and varied stimulus onset asynchrony (SOA) between the fourth (last) entrainer and the visual target (Fig 1D). We tested 11 SOAs, starting from $47.1 \mathrm{~ms}$, up to $282.4 \mathrm{~ms}$, in steps of $23.5 \mathrm{~ms}$ ( 2 frames). Note that the SOA at which the visual target was in-phase with the entrainers is at $94.1 \mathrm{~ms}$ for $10.6 \mathrm{~Hz}$-cueing and at $188.2 \mathrm{~ms}$ for $5.3 \mathrm{~Hz}$-cueing ( $3^{\text {rd }}$ versus $7^{\text {th }}$ tested SOA, Fig $1 \mathrm{D}$, see dashed annuli), and that our range of SOAs covered three cycles of an alpha-oscillation, allowing for detection of a possible cyclic pattern in behavioral performance at alpha-frequency. All conditions were presented in random order across trials in seven runs. A total of 1232 trials were sampled, resulting in 28 trials per condition cell per participant.

\section{Testing for a link to brain rhythms (experiment 2b)}

To evaluate the relationship between the cyclic pattern in visual performance and actual brain oscillations, we measured resting-state alpha oscillations (eyes open and closed, five minutes) using a 248-magnetometer whole-head MEG-system (MAGNES ${ }^{\circledR} 3600$ WH, 4-D Neuroimaging) in fourteen participants of experiment 2 who were available for this follow-up measurement. One subject who could not be measured with MEG was measured with EEG instead (8 parieto-occipital electrodes), bringing the total number of subjects to 15 . For simplicity we continue to refer to "MEG measurements" below. 


\section{Preprocessing behavioral data}

Only trials with reaction times between 200 and $1200 \mathrm{~ms}$ were included to remove outliers in both experiments. Hit rate (proportion correct) served as the dependent variable of interest.

\section{Analysis Experiment 1}

We implemented a full within-subjects design with factors Rhythmic cueing type (motion, flicker) $\mathrm{x}$ Congruency of cueing (congruent, incongruent) $\mathrm{x}$ Frequency of cueing (5 levels). Hit rate (proportion correct) per condition was subjected to repeatedmeasures Analysis of Variance (RM-ANOVAs, Huynh-Feldt corrected). Results were further explored using simple tests (ANOVAs) or 2-tailed paired-samples t-tests where appropriate, as indicated in the Results section.

\section{Analysis Experiment 2}

In experiment 2, we focused on the temporal profile of visual task performance over Delays (11 SOAs) between visual target onset and the last entrainer. To evaluate whether a cyclic pattern in visual task performance was apparent, we applied curve fitting procedures in custom software using robust nonlinear least-squares fitting in MATLAB. We analyzed group-averaged hit rate for all conditions separately $(10.6 \mathrm{~Hz}$ and $5.3 \mathrm{~Hz}$, spatially congruent and incongruent locations), after linearly detrending the data to remove linear effects across SOA and retain any cyclic patterns around the mean. We then fitted both $10 \mathrm{~Hz}$ and $5 \mathrm{~Hz}$ cosine curves to the data (fixed frequency, variable phase). R-squared values of the group mean data were statistically evaluated using bootstrapping. To this end, labels of the 11 SOAs were randomly permuted over 2500 iterations, and a model cosine $(10 \mathrm{~Hz}$ or $5 \mathrm{~Hz})$ was fitted to the resulting behavioral pattern each time, generating a null distribution of $2500 \mathrm{R}$-squared values. The Rsquared value obtained from the actual data was related to this created null-distribution to evaluate whether it fell in the top- $95^{\text {th }}$ percentile. If so, this by definition indicated that the model cosine significantly explained variance in the group data.

\section{Analysis Experiment $2 b$}

Using standard Fourier transforms over parieto-occipital sensors of the recorded MEG data, we could identify a clear peak in the alpha band $(8-12 \mathrm{~Hz})$ for each participant (individual alpha-frequency). We then fitted a model cosine (using robust nonlinear least-squares fitting) to the behavioral $10.6 \mathrm{~Hz}$-data of each of the 15 participants to extract the frequency that best reflects the individual behavioural patterns, identical to the analysis above (Exp 2) except that frequency was variable $(7-13 \mathrm{~Hz})$ and fitting was done on the individual level. Note that on the group level, a cosine always better fitted the detrended than the original data. On the individual level, detrending distorted the 
behavioural data in some participants who showed no strong trend but had outliers at the first or last position. Outliers affected the (non-robust) detrending but not the (robust) curve fitting. To avoid biased results, fitting was performed on both original and detrended data and the best-fitting result was used to extract the individual behavioural alpha peak. We then tested for a positive relationship between frequencies in behavioural and MEG data (Pearson correlation, one-tailed testing) to compare behavioral patterns with intrinsic brain oscillations.

\section{Results}

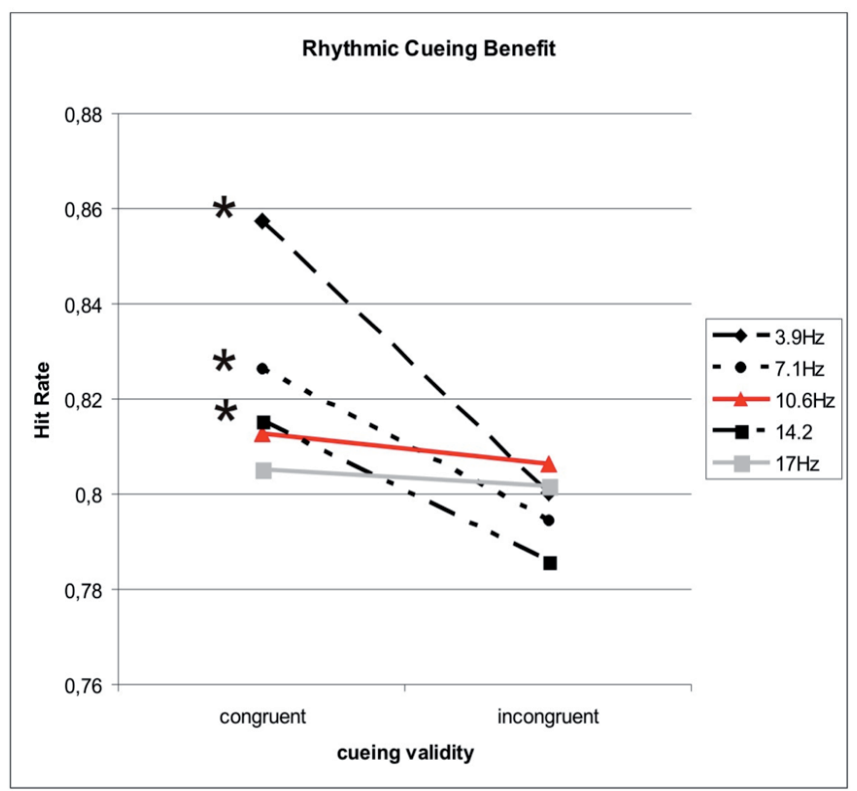

Figure 2: Results Experiment 1.

Group averaged hit rates for target detection at congruent and incongruent locations as a function of the five cueing frequencies (exploring a significant Congruency x Frequency interaction). In spite of spatially non-predictive cueing, significant cueing benefits (higher hit rate at cued relative to uncued locations) were observed for cueing at $3.9 \mathrm{~Hz}, 7.1 \mathrm{~Hz}$, and $14.2 \mathrm{~Hz}$ but broke down for intermediate $10.6 \mathrm{~Hz}$ cueing in the alpha-band $(8-12 \mathrm{~Hz})$. At the highest frequency of $17 \mathrm{~Hz}$, rhythmic cueing no longer led to cueing benefits. *: significant cueing benefits at $\mathrm{p}<0.05$.

\section{Alpha-specific breakdown of cueing benefits (experiment 1)}

We investigated spatial cueing benefits from attentional mechanisms associated with rhythmic (entrainment) cues as a function of cueing frequency in two entrainment 
conditions (flicker vs. motion, Fig 1A-B). We tested this by assessing visual task performance (target discrimination) at the first time-point in phase with the preceding entrainer-cues (Fig 1C). The overall repeated-measures ANOVA on hit rate with factors Rhythmic Cueing Type (flicker vs. motion), Congruency of cueing (congruent vs. incongruent), and Frequency of cueing $(3.9,7.1,10.6,14.2$ vs. 17Hz) showed a main effect of Rhythmic Cueing Type $(\mathrm{F}=7.01, \mathrm{P}<0.05)$. Performance was weakly but significantly better with motion cueing (hit rate=0.82) than flicker cueing (hit rate $=0.80$ ). This is likely due to a forward masking of the visual target by the final cue, which occurs in the flicker, but not in the motion condition. Importantly, Rhythmic Cueing Type did not interact with any other condition (3-way interaction: $F=0.42$, $\mathrm{P}=0.792$, 2-way interaction with Congruency: $\mathrm{F}=0.12, \mathrm{P}=0.728$, 2-way interaction with Frequency: $\mathrm{F}=1.45, \mathrm{P}=0.260$ ). In other words, all the effects we are about to describe were statistically equivalent for both types of cueing, and therefore likely to be independent of masking.

The overall ANOVA revealed a main effect of Congruency of cueing $(\mathrm{F}=8.44$, $\mathrm{P}<0.01$ ), with better visual task performance at cued than uncued locations (cueing benefit), despite rhythmic cueing being spatially non-predictive regarding upcoming target position, and therefore possibly due to exogenous attention mechanisms driven by the cues. Importantly, this effect was dependent on the Frequency of cueing $(\mathrm{F}=4.78$, $\mathrm{P}<0.01$ ), showing that attentional entrainment was not equally effective over all frequencies. Figure 2 illustrates visual task performance for congruent and incongruent cueing across all frequencies, collapsed over flicker and motion cueing (due to absence of a 3-way interaction, see Table 1 for noncollapsed data across all conditions). A cueing benefit with better performance at cued than uncued location is observed for rhythmic cueing at $3.9 \mathrm{~Hz}$ (simple test for congruency effect: $\mathrm{P}<0.001), 7.1 \mathrm{~Hz}(\mathrm{P}<0.05)$ and $14.2 \mathrm{~Hz}(\mathrm{P}<0.05)$, hence a broad range of frequencies, but not for the $10.6 \mathrm{~Hz}$ $(\mathrm{P}=0.662)$ or the $17 \mathrm{~Hz}(\mathrm{P}=0.783)$ condition. There is thus a discontinuity of cueing benefit with alpha-stimulation compared to flanker frequencies. Entrainment effects on perceptual behavior are indeed frequency-specific.

\begin{tabular}{|c|c|c|c|c|c|c|c|c|}
\hline & \multicolumn{4}{|l|}{ Flicker Cueing } & \multicolumn{4}{|l|}{ Motion Cueing } \\
\hline & Cue Left & & Cue Right & & Cue Left & & Cue Right & \\
\hline & Target Left & Target Right & Target Left & Target Right & Target Left & Target Right & Target Left & Target Right \\
\hline $3.9 \mathrm{~Hz}$ & $0.858[0.076]$ & $0.781[0.090]$ & $0.777[0.091]$ & $0.845[0.079]$ & $0.832[0.082]$ & $0.867[0.074]$ & $0.864[0.075]$ & $0.819[0.084]$ \\
\hline $7.1 \mathrm{~Hz}$ & $0.806[0.086]$ & $0.806[0.086]$ & $0.789[0.089]$ & $0.840[0.080]$ & $0.795[0.088]$ & $0.861[0.076]$ & $0.799[0.087]$ & $0.786[0.090]$ \\
\hline $10.6 \mathrm{~Hz}$ & $0.804[0.087]$ & $0.785[0.090]$ & $0.797[0.088]$ & $0.802[0.087]$ & $0.804[0.087]$ & $0.836[0.081]$ & $0.804[0.087]$ & $0.833[0.082]$ \\
\hline $14.2 \mathrm{~Hz}$ & $0.805[0.086]$ & $0.784[0.090]$ & $0.757[0.094]$ & $0.801[0.087]$ & $0.825[0.083]$ & $0.835[0.081]$ & $0.816[0.085]$ & $0.775[0.091]$ \\
\hline $17 \mathrm{~Hz}$ & $0.817[0.084]$ & $0.785[0.090]$ & $0.788[0.089]$ & $0.762[0.093]$ & $0.828[0.082]$ & $0.823[0.083]$ & $0.811[0.085]$ & $0.801[0.087]$ \\
\hline
\end{tabular}

\section{Periodicity in visual task performance at $10 \mathrm{~Hz}$ frequency (experiment 2)}

In experiment 2 we tested effects of cueing at $10.6 \mathrm{~Hz}$ and at the first alpha-subharmonic (i.e. $5.3 \mathrm{~Hz}$ ). If phase-locking of naturally occurring alpha oscillations drives our results, this alpha-subharmonic should have similar effects as $10.6 \mathrm{~Hz}$-cueing (although possibly to a lesser extent), since the $5.3 \mathrm{~Hz}$ condition constitutes a 'weak' $10.6 \mathrm{~Hz}$ entrainer rhythm with every second entrainer left out. As in experiment $1,10.6 \mathrm{~Hz}$-cueing in 
experiment 2 did not result in a cueing benefit $(\mathrm{P}=0.133)$, nor did alpha-subharmonic cueing at $5.3 \mathrm{~Hz}(\mathrm{P}=0.293)$.

If $10.6 \mathrm{~Hz}$-cueing indeed leads to phase-locking of underlying alphaoscillations in the brain and the phase of this oscillation is functionally relevant for visual task performance, then visual task performance should cycle over time post-train at alpha-frequency, in synchrony with the entrained alpha-oscillation. We tested target discrimination over a window of $\sim 300 \mathrm{~ms}$ after the last entrainer ( 3 alpha cycles, 11 SOAs), using only motion entrainment. Figure 3 illustrates the time-course of visual task performance after $10.6 \mathrm{~Hz}$ - and $5.3 \mathrm{~Hz}$-cueing for spatially congruent targets (after linear detrending, see Table 2 for original data), with the best-fitting $10 \mathrm{~Hz}$ cosine models superimposed.

A $10.6 \mathrm{~Hz}$ congruent cueing

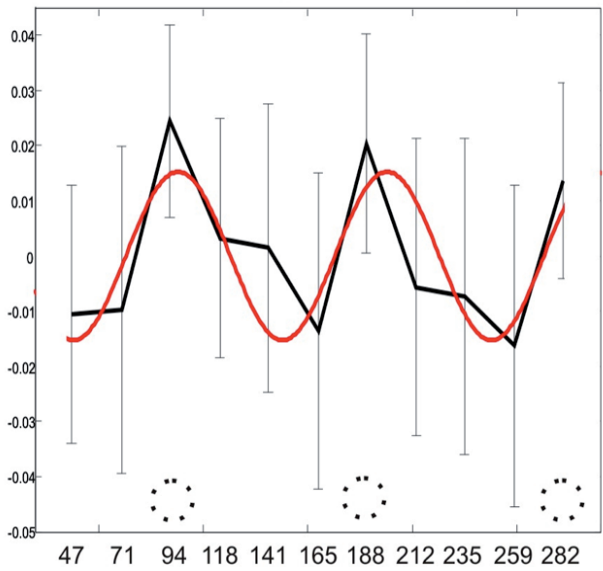

B $\quad 5.3 \mathrm{~Hz}$ congruent cueing

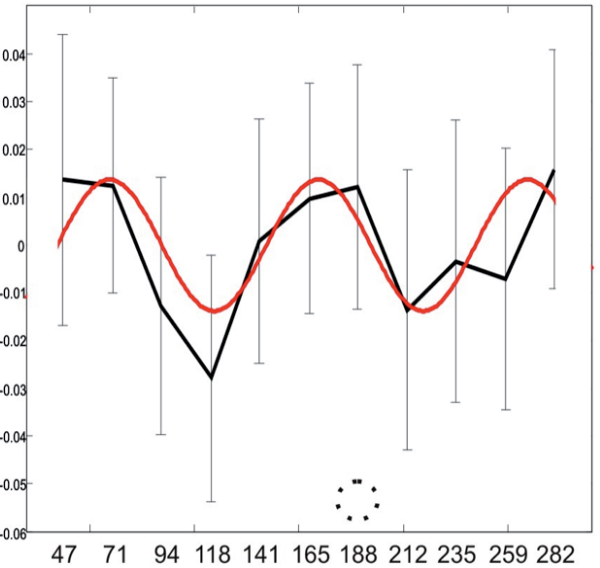

Figure 3: Results Experiment 2.

Group averaged hit rate (linearly detrended) at congruent locations over 3 alpha cycles posttrain following A) $10.6 \mathrm{~Hz}$ - or B) $5.3 \mathrm{~Hz}$-cueing. The best fitting $10 \mathrm{~Hz}$ model cosines are superimposed. For both $10.6 \mathrm{~Hz}$ and $5.3 \mathrm{~Hz}$ (subharmonic) congruent cueing, we found recurrent peaks and troughs which were significantly fitted by a $10 \mathrm{~Hz}$ cosine function. Thus, a cyclic pattern at alpha-frequency is apparent in visual task performance. Error bars reflect standard error of the mean.

Visual inspection clearly reveals a cyclic pattern of performance peaks after $10.6 \mathrm{~Hz}$ motion cueing (Fig 3A). Moreover, the peaks in this cyclic pattern are exactly in-phase with the preceding rhythmic cues, and the periodic pattern seems to span over at least three $10 \mathrm{~Hz}$ cycles (i.e. presenting three recurrent performance peaks rather than only one confined to the first in-phase SOA, 94.1ms). Curve fitting procedures and permutation tests (see Methods) revealed that a $10 \mathrm{~Hz}$ cosine model significantly fitted the $10.6 \mathrm{~Hz}$ group data $(57 \%$ explained variance, bootstrapped $95 \%$-cut-off at $53 \%$ ), statistically confirming the presence of a $10 \mathrm{~Hz}$ cyclic pattern at the cued position. For comparison, $10 \mathrm{~Hz}$ cosine models could not explain performance at the incongruent position ( $7 \%$ explained variance: cut-off $54 \%$ ), nor was performance explained by $5 \mathrm{~Hz}$ 
models at congruent (15\% explained variance: cut-off $52 \%)$ or incongruent positions (23\% explained variance: cut-off $51 \%)$.

Cueing at $5.3 \mathrm{~Hz}$ led to similar results at cued positions (Fig 3B). Visual inspection again reveals an oscillatory pattern with 3 peaks. Statistically, a $10 \mathrm{~Hz}$ cosine model significantly explained performance fluctuations in the group curve in this congruent condition, despite $5.3 \mathrm{~Hz}$ cueing (54\% variance explained, cut-off: $53 \%$ ). For comparison, fitting a $5 \mathrm{~Hz}$ cosine wave to these data did not explain its variance $(6 \%$ explained, cut-off: $52 \%$ ), nor was the variance in the incongruent condition explained by $10 \mathrm{~Hz}$ fitting $(24 \%$ explained variance, cut-off $51 \%)$ or $5 \mathrm{~Hz}$ fitting ( $10 \%$ explained variance: cut-off $50 \%$ ). In short, there was more evidence for a $10 \mathrm{~Hz}$ than a $5 \mathrm{~Hz}$ induced wave in the behavioural data when entrainment was at $5.3 \mathrm{~Hz}$. Moreover, just as in the $10.6 \mathrm{~Hz}$ cueing condition, this effect was specific to the spatially congruent condition.

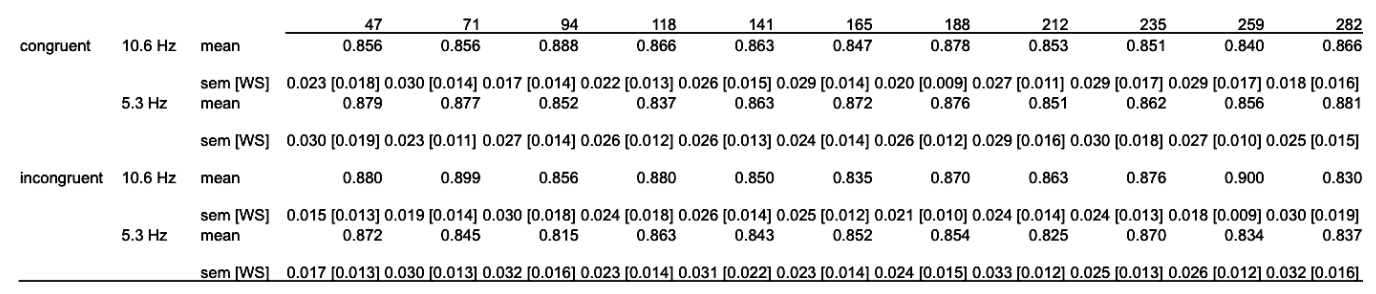

Table 2: results of experiment 2 (outliers removed), before detrending. Displayed are the average proportion correct per condition, per SOA (horizontal direction).

Table 2: results of experiment 2 (outliers removed), before detrending. Displayed are the average proportion correct per condition
Also shown are standard error of the mean (SEM), before and [after removal of between-subject variability (within-subject: WS)]

\section{Oscillations in visual task performance are linked to occipito-parietal}

\section{brain rhythms (Experiment 2b)}

To link periodicity in visual performance to intrinsic alpha-oscillations in the brain, we tested for a positive correlation between the best fitting frequency in individual behavioural data (cosine model, $10.6 \mathrm{~Hz}$ condition) and the individual alpha-frequency over occipito-parietal areas in resting-state MEG measurement (obtained for 15 participants of Experiment 2, see Methods). Figure 4 shows the resulting scatterplot and regression result. Although we used only four entrainers (and did not tune stimulation frequency to individual alpha-oscillations, i.e. fixed to $10.6 \mathrm{~Hz}$ ), the individual frequency in task performance significantly correlated with the frequency of the intrinsic alpha-oscillation obtained in the same participant on a different day $(r=0.61$, $\mathrm{P}<0.01)$. 


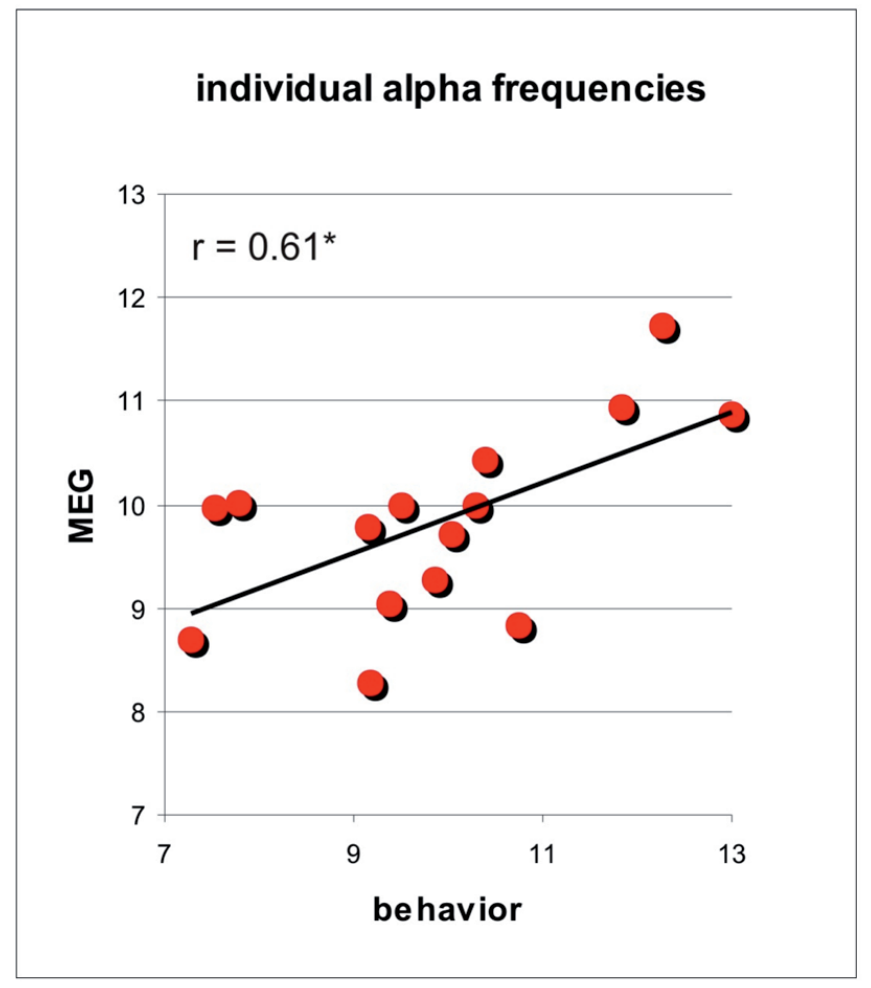

Figure 4: Behavior-MEG correlation.

Scatterplot and linear regression analysis between frequency of rhythms in visual task performance (Experiment 2, x-axis) and MEG (recorded in a subset of participants, yaxis). The behavioural cyclic pattern showed a significant positive correlation with individual resting occipito-parietal alpha frequency. This suggests that the cyclic pattern in behaviour (shown for the group results in Figure 3) may reflect entrainment of intrinsic brain oscillations in the alpha-band.

\section{Discussion}

The results of experiment 1 reveal broadband rhythmic cueing benefits on visual target detection for a frequency range between at least $\sim 4-14 \mathrm{~Hz}$ (in line with previous reports, (Doherty, Rao et al. 2005; Mathewson, Fabiani et al. 2010; Rohenkohl, Coull et al. 2011; Rohenkohl and Nobre 2011; Mathewson, Prudhomme et al. 2012), discussed below), with the exception of $10.6 \mathrm{~Hz}$ cueing where benefits broke down. The latter frequency is centered in the alpha-range $(8-12 \mathrm{~Hz})$, a prominent inhibitory rhythm of the posterior brain (Klimesch, Sauseng et al. 2007). In the second experiment, we reproduced the break-down of cueing benefits for $10.6 \mathrm{~Hz}$-stimulation and subharmonical (5.3hz) cueing, and revealed an alpha-rhythm in visual task performance over 3 cycles post-cueing, which was also present after $5.3 \mathrm{~Hz}$ entrainment, and correlated in frequency with individual resting alpha-oscillations over occipito-parietal 
areas. The cyclic pattern in visual task performance at frequencies of intrinsic rhythms following stimulation with these frequencies not only suggests that these oscillations have been entrained (Herrmann 2001), but also that they are functionally relevant and that entrainment paradigms can be used to study the role of oscillations in behaviour (Thut, Schyns et al. 2011; Vanrullen and Dubois 2011).

\section{Insights into the role of oscillatory brain activity in attention and}

\section{perception from alpha-specificity and alpha-periodicity of behavioural}

\section{effects to rhythmic entrainment}

Experiment 1 shows that the entrainment/cueing effects on target detection are frequency-specific. Cueing benefits, which are generally broadband, can break down for stimulation at alpha-frequency $(10.6 \mathrm{~Hz})$, or its subharmonic $(5.3 \mathrm{~Hz})$. It has recently been shown that rhythmic motion cueing engages automatic attention processes (Rohenkohl, Coull et al. 2011) which involve the downregulation of parieto-occipital alpha-power $(8-12 \mathrm{~Hz})$ time-locked to the expected target onset (shown for $1.25 \mathrm{~Hz}$ - and $2.5 \mathrm{~Hz}$-cueing by (Rohenkohl and Nobre 2011)). This would help to explain why $10.6 \mathrm{~Hz}$-cueing did not convey a benefit in our study, while benefits for flanker frequencies outside the alpha-band $(7.1 / 14.2 \mathrm{~Hz})$ were evident. With $10.6 \mathrm{~Hz}$-cueing, the rhythmic train may have phase-locked (synchronized) the intrinsic alpha-oscillations (corresponding to entrainment of oscillatory brain activity in the alpha-band, (Thut, Schyns et al. 2011)) which would likely interfere with the attention-related alphadesynchronization needed for conferring perceptual benefits (Rohenkohl and Nobre 2011). Alpha-synchronization (by the rhythmic train) counteracting task-relevant (attention-related) alpha-desynchronization form a speculative but parsimonious mechanistic account of our results. Cueing at the alpha subharmonic frequency of $5.3 \mathrm{~Hz}$ also yielded no cueing benefits (experiment 2), despite strong cueing benefits at flanker frequencies $(3.9 / 7.1 \mathrm{~Hz}$, in experiment 1$)$. This further supports our conjecture of entrainment of brain rhythms in the alpha band, and seems difficult to explain otherwise. These frequency-specific effects therefore point to a special role for alphaoscillations in visual perception.

One view holds that visual brain oscillations may implement a periodic sampling mechanism for perception (VanRullen and Koch 2003; VanRullen, Reddy et al. 2005; VanRullen, Carlson et al. 2007; Mathewson, Lleras et al. 2011) with enhanced visual performance at one preferred phase of the oscillatory cycle and reduced performance at the opposite phase. Yet, only a few studies we are aware of have attempted directly to entrain intrinsic alpha oscillations (cf (Thut, Schyns et al. 2011)) in order to study phase-dependence of perception over several alpha cycles. Previous reports of perceptual benefits from rhythmic cueing (Doherty, Rao et al. 2005; Mathewson, Fabiani et al. 2010; Rohenkohl, Coull et al. 2011; Rohenkohl and Nobre 2011) could generally be explained by a top-down driven cognitive anticipation process, even when cueing effects or neural correlates thereof are recurrent (show a cyclic pattern) (Rohenkohl and Nobre 2011; Mathewson, Prudhomme et al. 2012) 
because the rhythmic cueing pattern per se generates expectations for cyclic reoccurrence of events. In contrast, top-down driven anticipation cannot explain the periodicity in perception in our results (experiment 2), for two reasons. First, after rhythmic stimulation with the alpha-subharmonic $5.3 \mathrm{~Hz}$, there was an alpha oscillation in visual task performance over time, whereas a top-down driven cognitive anticipation process would have yielded a $5.3 \mathrm{~Hz}$ pattern. Second, across participants, the individual, peak alpha frequencies in visual task performance correlated with peak alpha frequencies in resting state MEG. The intrinsic alpha oscillations seem therefore to underlie the behavioral alpha oscillations, further ruling out anticipation as a possible explanation. Our results therefore strongly suggest that a rhythmic train of visual cues at alpha frequency $(10.6 \mathrm{~Hz})$ can reveal a rhythmic pattern in visual task performance driven by alpha oscillation in visual areas.

\section{Insights into the mechanics of exogenous spatiotemporal attention}

\section{processes}

Previous studies implemented rhythmic cueing paradigms similar to those we employed to study the mechanisms and constraints of attention processes (Doherty, Rao et al. 2005; Mathewson, Fabiani et al. 2010; Rohenkohl, Coull et al. 2011; Rohenkohl and Nobre 2011; Mathewson, Prudhomme et al. 2012). Behavioral effects to flicker- or motion-cues consist of enhanced perception of targets appearing at expected time-points or positions, i.e. in temporal and/or spatial alignment with the preceding rhythmic cues, for stimulation at $1.8 \mathrm{~Hz}$ (Doherty, Rao et al. 2005), $2.5 \mathrm{~Hz}$ (Rohenkohl, Coull et al. 2011; Rohenkohl and Nobre 2011), and 12.1Hz (Mathewson, Fabiani et al. 2010; Mathewson, Prudhomme et al. 2012), in line with the broadband cueing benefit we observed ( $\sim 4-14 \mathrm{~Hz})$. In contrast to previous studies on attention using rhythmic cueing (e.g., (Doherty, Rao et al. 2005)), we did not manipulate predictability of targets at spatial positions, i.e. targets were equally likely to occur at cued or uncued locations. Although not at all spatially predictive (but fully temporally predictive), the rhythmic trains of cues did improve visual target processing at the cued relative to the uncued locations. We therefore interpret our spatial cueing benefit to result from automatic exogenous visuospatial attention mechanisms, rather than endogenous attention control. This is in line with recent findings by Rohenkohl et al. (Rohenkohl, Coull et al. 2011; Rohenkohl and Nobre 2011) who showed that the rhythm in periodic visual stimulation likely activates automatic exogenous temporal attention mechanisms, while (here absent) symbolic information can affect endogenous temporal attention mechanisms. Overall, our data confirm the notion that during rhythmic cueing, temporal expectations cooperate with visual spatial attention for optimizing perceptual analysis at/towards cued locations (Doherty, Rao et al. 2005) over a broadband of stimulation frequencies (Mathewson, Fabiani et al. 2010; Rohenkohl, Coull et al. 2011; Rohenkohl and Nobre 2011; Mathewson, Prudhomme et al. 2012), and that this is under automatic attention control (Rohenkohl, Coull et al. 2011).

In addition, our findings provide information on possible dynamic limits of these attention processes. Apart from the breakdown of benefits at alpha-rhythms, the 
frequency of cueing differentially affected perception in such a way as to suggest that automatic attention processes with dynamic limits are at play (highest cueing benefit at lowest frequency $=3.9 \mathrm{~Hz}$, decreasing cueing benefit as frequency of stimulation increases). Towards an upper dynamic limit, spatial and temporal attention may be progressively less able to engage, disengage and reallocate to present and future events of the rhythmic event sequence to finally break off beyond this limit. Recent work has revealed a lower dynamic limit at around $1 \mathrm{~Hz}$, since with overly long delays between entrainers and targets $(1400 \mathrm{~ms}, 0.7 \mathrm{~Hz})$ cueing benefits disappear (e.g. (Correa and Nobre 2008; Rohenkohl and Nobre 2011). Our study points towards an upper dynamic limit at $14-17 \mathrm{~Hz}$, as indicated by the lack of cueing effects at $17 \mathrm{~Hz}$ (the highest frequency we tested). However, it remains unclear whether these limits reflect a fundamental (absolute) upper/lower limit of the attention system, or whether these limits depend on the number of entrainers and the particular paradigm, as attention allocation may become more accurate and effective when more than four entrainers are employed (see (Ariga and Yokosawa 2008). In future work, it would be of interest to delineate in more detail the exact constraints of rhythmic attentional cueing benefits through more detailed parametric manipulations.

\section{Limitations and caveats}

The most likely collective account of our data set is that entrainment of brain rhythms in the alpha band contributed to our results, because it can explain both alpha-specificity and alpha-periodicity of behavioral effects (experiment 1 and 2) in light of the link to posterior alpha oscillations (experiment 2b). Yet, although less likely, alternative explanations should be considered.

Since the delays between cues within the trains as well as between the last entrainer and visual target were different across frequencies of cueing (by design), we need to consider whether the results of experiment 1 (i.e. alpha-specificity) could have resulted from differential forward masking effects across conditions, differential attentional blinks associated with the rapid serial visual presentation per frequency, or differential apparent motion effects. First, forward masking effects (due to the last entrainer masking the visual target) may differentially interfere with perception depending on presentation frequency, as the cue-target delay (mask-target SOA) does affect the strength of masking (Breitmeyer and Ogmen 2006). Yet, since in the motion entrainment condition visual targets were not in the same location as the visual entrainers (the potential masks) and since motion entrainment did not differ from flicker entrainment in terms of frequency effects (no interaction between Rhythmic Cueing Type and Frequency of cueing), forward masking seems unlikely to explain our results. Second, the attentional blink may differentially affect perception across presentation frequencies because it also depends on SOA (Shapiro, Raymond et al. 1997). Yet, such an explanation would be difficult to reconcile with the pattern of cueing effects we observe across frequencies (in particular with the absence of benefits at two frequencies $5.3 \mathrm{~Hz}$ and $10.6 \mathrm{~Hz}$, but the intermediate frequency $7.1 \mathrm{~Hz}$ being unaffected). We do not see how this pattern can be interpreted other than in terms of entrained alphaoscillations. Third and in analogy to the above, although apparent motion depends on 
stimulation frequency (Ekroll, Faul et al. 2008), our pattern of findings with breakdowns of cueing in $5.3 \mathrm{~Hz}$ and $10.6 \mathrm{~Hz}$ but not at $7.1 \mathrm{~Hz}$ seems difficult to explain in this context.

A limitation of our experiments is that we did not record MEG simultaneously with task performance. This means that the mechanism of alpha phase alignment explaining our results, although parsimonious and in line with previous work (see above and Introduction), remains speculative. A recent study by Mathewson et al. (Mathewson, Prudhomme et al. 2012) however provides empirical support for this assumption. These authors measured electroencephalography (EEG) while rhythmic trains of visual stimuli preceded a visual target. Behaviorally, targets in phase with rhythmic trains were detected more often than targets out of phase. The EEG showed phase-alignment of alpha oscillations to the rhythmic train, and this increase in phaselocking predicted the increase in target detection when targets were in phase. These results demonstrate that rhythmic stimulation at alpha frequency could indeed align alpha oscillations which might be functionally relevant for visual performance (Mathewson, Gratton et al. 2009; Mathewson, Fabiani et al. 2010; Mathewson, Lleras et al. 2011; Mathewson, Prudhomme et al. 2012), supporting the interpretation of our findings here. In contrast to our findings, individual peak alpha frequencies (in resting state EEG) did not correlate to any of the behavioral measures in Mathewson et al. (Mathewson, Prudhomme et al. 2012). Also, the experimental design and behavioral analysis did not allow a direct evaluation of whether behavior oscillated at alpha frequency, and whether entrainment effects are frequency- and location-specific. Our results and those described in Mathewson et al. (Mathewson, Prudhomme et al. 2012) are therefore highly complementary. Taken together, they strongly support the hypothesis that rhythmic visual stimulation phase-aligns intrinsic alpha oscillations that are directly relevant for successful visual perception.

\section{Alpha phase alignment by attentional events?}

One last aspect of our results that affords further consideration involves the spatial configuration of our stimuli in the motion entrainment condition. We showed that the entrainment effects are spatially specific, in that only congruent cueing resulted in cyclic patterns in perception at alpha frequency (experiment 2). Yet, all the entrainers of this (motion) condition were positioned in the hemifield opposite to the target location with the final entrainer always presented at midline directly underneath the fixation cross. Therefore, if visual entrainers at alpha-rhythm would phase-align alpha oscillations in corresponding retinotopic locations, the oscillatory pattern of behavior should actually have been apparent in the incongruent condition, where visual cues and targets were in the same hemifield, rather than in the congruent condition where the target appeared on the other side. This was clearly not the case, which raises the question whether some of our results may be explained by resetting (phase-locking) of perception (or alpha oscillations) to specific time points via attentional mechanisms driven by the rhythmic cues.

Landau and Fries (Landau and Fries 2012) recently showed that attentional capture in one hemifield can lead to phase-locked behavioral oscillations in the same 
and opposite hemifield. Our results seem to extend this finding in an interesting way. Phase-locked behavioural oscillations were observed in the hemifield of anticipated target location, not in the hemifield of physical visual stimulation, i.e. where the visual cues actually appeared. The phase-locking in our experiment must therefore have been generated by an attentional, rather than sensory, signal, albeit likely of automatic nature (as the cue train was not spatially predictive). Thus, while Landau and Fries (Landau and Fries 2012) already showed that attention captured to one hemifield can have oscillatory behavioral effects in the opposite hemifield, our results demonstrate that such attentional capture can occur without any stimulation in this opposite hemifield and possibly involves natural alpha oscillations in the brain.

\section{Conclusions}

Our results demonstrate alpha-specificity and alpha-periodicity of behavioural effects to rhythmic cueing paradigms, correlating with brain oscillations as measured by MEG. They reveal the functional relevance of intrinsic alpha-oscillations in successful visual perception, and demonstrate that these oscillations can be controlled (and thus studied) by rhythmic visual stimulation at intrinsic frequencies. 


\section{References}

Ariga, A. and K. Yokosawa (2008). "Attentional awakening: gradual modulation of temporal attention in rapid serial visual presentation." Psychol Res 72(2): 192-202.

Breitmeyer, B. and H. Ogmen (2006). Visual Masking: time slices through conscious and unconscious vision. New York, Oxford University press.

Busch, N. A., J. Dubois, et al. (2009). "The phase of ongoing EEG oscillations predicts visual perception." J Neurosci 29(24): 7869-7876.

Buzsaki, G. (2006). Rhythms of the Brain. New York, Oxford University Press.

Correa, A. and A. C. Nobre (2008). "Neural modulation by regularity and passage of time." J Neurophysiol 100(3): 1649-1655.

Ding, J., G. Sperling, et al. (2006). "Attentional modulation of SSVEP power depends on the network tagged by the flicker frequency." Cerebral cortex 16(7): 1016-1029.

Doherty, J. R., A. Rao, et al. (2005). "Synergistic effect of combined temporal and spatial expectations on visual attention." J Neurosci 25(36): 8259-8266.

Drewes, J. and R. VanRullen (2011). "This is the rhythm of your eyes: the phase of ongoing electroencephalogram oscillations modulates saccadic reaction time." The Journal of neuroscience : the official journal of the Society for Neuroscience 31(12): 4698-4708.

Ekroll, V., F. Faul, et al. (2008). "Classification of apparent motion percepts based on temporal factors." Journal of vision 8(4): 31 31-22.

Fiebelkorn, I. C., J. J. Foxe, et al. (2011). "Ready, set, reset: stimulus-locked periodicity in behavioral performance demonstrates the consequences of cross-sensory phase reset." J Neurosc 31(27): 9971-9981.

Foxe, J. J. and A. C. Snyder (2011). "The Role of Alpha-Band Brain Oscillations as a Sensory Suppression Mechanism during Selective Attention." Front Psychol 2: 154.

Galambos, R., S. Makeig, et al. (1981). "A 40-Hz auditory potential recorded from the human scalp." Proc Natl Acad Sci U S A 78(4): 2643-2647.

Hamm, J. P., K. A. Dyckman, et al. (2010). "Preparatory activations across a distributed cortical network determine production of express saccades in humans." The Journal of neuroscience : the official journal of the Society for Neuroscience 30(21): 7350-7357.

Hanslmayr, S., A. Aslan, et al. (2007). "Prestimulus oscillations predict visual perception performance between and within subjects." Neuroimage 37(4): 1465-1473.

Herrmann, C. S. (2001). "Human EEG responses to 1-100 Hz flicker: resonance phenomena in visual cortex and their potential correlation to cognitive phenomena." Exp Brain Res 137(3-4): 346-353.

Jones, M. R., H. Moynihan, et al. (2002). "Temporal aspects of stimulus-driven attending in dynamic arrays." Psychol Sci 13(4): 313-319.

Klimesch, W., P. Sauseng, et al. (2007). "EEG alpha oscillations: the inhibition-timing hypothesis." Brain Research Reviews 53(1): 63-88.

Lakatos, P., G. Karmos, et al. (2008). "Entrainment of neuronal oscillations as a mechanism of attentional selection." Science 320(5872): 110-113.

Landau, A. N. and P. Fries (2012). "Attention samples stimuli rhythmically." Current biology 22(11): 1000-1004. 
Mathewson, K. E., M. Fabiani, et al. (2010). "Rescuing stimuli from invisibility: Inducing a momentary release from visual masking with pre-target entrainment." Cognition 115(1): 186-191.

Mathewson, K. E., G. Gratton, et al. (2009). "To see or not to see: prestimulus alpha phase predicts visual awareness." J Neurosci 29(9): 2725-2732.

Mathewson, K. E., A. Lleras, et al. (2011). "Pulsed out of awareness: EEG alpha oscillations represent a pulsed-inhibition of ongoing cortical processing." Front Psychol 2: 99.

Mathewson, K. E., C. Prudhomme, et al. (2012). "Making Waves in the Stream of Consciousness: Entraining Oscillations in EEG Alpha and Fluctuations in Visual Awareness with Rhythmic Visual Stimulation." Journal of cognitive neuroscience.

Monto, S., S. Palva, et al. (2008). "Very slow EEG fluctuations predict the dynamics of stimulus detection and oscillation amplitudes in humans." J Neurosci 28(33): 82688272.

Rohenkohl, G., J. T. Coull, et al. (2011). "Behavioural dissociation between exogenous and endogenous temporal orienting of attention." PLoS One 6(1): e14620.

Rohenkohl, G. and A. C. Nobre (2011). "Alpha oscillations related to anticipatory attention follow temporal expectations." J Neurosci 31(40): 14076-14084.

Romei, V., V. Brodbeck, et al. (2008). "Spontaneous fluctuations in posterior alphaband EEG activity reflect variability in excitability of human visual areas." Cereb Cortex 18(9): 2010-2018.

Romei, V., J. Gross, et al. (2010). "On the role of prestimulus alpha rhythms over occipito-parietal areas in visual input regulation: correlation or causation?" J Neurosci 30(25): 8692-8697.

Romei, V., J. Gross, et al. (2012). "Sounds reset rhythms of visual cortex and corresponding human visual perception." Current biology 22(9): 807-813.

Schroeder, C. E., D. A. Wilson, et al. (2010). "Dynamics of Active Sensing and perceptual selection." Curr Opin Neurobiol 20(2): 172-176.

Shang, C. F., Y. Dan, et al. (2011). "Periodic stimulation induces long-range modulation of cortical responses and visual perception." J Physiol 589(Pt 13): 31253133.

Shapiro, K. L., J. E. Raymond, et al. (1997). "The attentional blink." Trends in cognitive sciences 1(8): 291-296.

Thut, G., A. Nietzel, et al. (2006). "Alpha-band electroencephalographic activity over occipital cortex indexes visuospatial attention bias and predicts visual target detection." J Neurosci 26(37): 9494-9502.

Thut, G., P. G. Schyns, et al. (2011). "Entrainment of perceptually relevant brain oscillations by non-invasive rhythmic stimulation of the human brain." Front Psychol 2: 170.

van Dijk, H., J. M. Schoffelen, et al. (2008). "Prestimulus oscillatory activity in the alpha band predicts visual discrimination ability." J Neurosci 28(8): 1816-1823.

VanRullen, R., T. Carlson, et al. (2007). "The blinking spotlight of attention." PNAS 104(49): 19204-19209.

Vanrullen, R. and J. Dubois (2011). "The psychophysics of brain rhythms." Front Psychol 2: 203. 
VanRullen, R. and C. Koch (2003). "Is perception discrete or continuous?" Trends in cognitive sciences 7(5): 207-213.

VanRullen, R. and J. S. Macdonald (2012). "Perceptual echoes at $10 \mathrm{~Hz}$ in the human brain." Current biology 22(11): 995-999.

VanRullen, R., L. Reddy, et al. (2005). "Attention-driven discrete sampling of motion perception." PNAS 102(14): 5291-5296. 



\title{
III.4
}

\section{Brain Network Dynamics Underlying}

\section{Visuospatial Judgment: an fMRI}

\author{
connectivity study
}

Related publication(s):

De Graaf, TA, Roebroeck, A, Goebel, R, Sack, AT (2010). Brain network dynamics underlying visuospatial judgment: an fMRI connectivity study. Journal of Cognitive Neuroscience. 22:9, pp. 2012-2026 


\begin{abstract}
Previous functional imaging research has consistently indicated involvement of bilateral fronto-parietal networks during the execution of visuospatial tasks. Studies with TMS have suggested that the right hemispheric network, but not the left, is functionally relevant for visuospatial judgments. However, very little is still known about the interactions within these fronto-parietal networks underlying visuospatial processing. In the current study, we investigated task modulation of functional connectivity (instantaneous correlations of regional time courses), and task-specific effective connectivity (direction of influences), within the right fronto-parietal network activated during visuospatial judgments. Ten healthy volunteers performed a behaviorally controlled visuospatial judgment task (ANGLE) or a control task (COLOR) in an fMRI experiment. Visuospatial task-specific activations were found in posterior parietal cortex (PPC) and middle/inferior frontal gyrus (MFG). Functional connectivity within this network was taskmodulated, with significantly higher connectivity between PPC and MFG during ANGLE than during COLOR. Effective connectivity analysis for directed influence revealed that visuospatial task-specific projections within this network were predominantly in a frontal-toparietal direction.
\end{abstract}




\section{Introduction}

Visuospatial processing refers to the spatial perception, recognition, and analysis of visual input. Visuospatial judgment is one example of higher-order visuospatial processing. It can involve the analysis of spatial features of visual stimuli such as distances, angles, or more generally spatial relations, between stimulus parts or aspects of visual images. In visuospatial judgment such analysis is, by definition, goal-related. It therefore often involves a focus on certain spatial features that are relevant to a particular task or goal. Visuospatial judgment is essential to human interaction with the environment.

To understand its neural correlate in the brain, extensive research has been done using functional neuroimaging methods such as positron emission tomography (Mellet et al., 1996; Haxby et al., 1991) and functional magnetic resonance imaging (fMRI; Goebel, Linden, Lanfermann, Zanella, \& Singer, 1998; Cohen et al., 1996), both providing measures of metabolic activity in restricted brain regions. This research has revealed network correlates of visuospatial tasks (Trojano et al., 2000; Goebel et al., 1998), visuospatial imagery (Sack, Camprodon, Pascual-Leone, \& Goebel, 2005; Formisano et al., 2002; Trojano et al., 2002; Mellet et al., 1996), visuospatial attention (Luks, Sun, Dale, Miller, \& Simpson, 2008; Giessing, Fink, Rosler, \& Thiel, 2007; Husain \& Nachev, 2007; Mayer, Seidenberg, Dorflinger, \& Rao, 2004; Corbetta, Kincade, \& Shulman, 2002), visuospatial working memory (Klingberg, 2006; Scherf, Sweeney, \& Luna, 2006; Klingberg, Forssberg, \& Westerberg, 2002; Postle, Berger, Taich, \& DðEsposito, 2000; Petit, Courtney, Ungerleider, \& Haxby, 1998), and visuomotor control (Culham, Cavina-Pratesi, \& Singhal, 2006; de Lange, Hagoort, \& Toni, 2005). All of these different functional imaging studies converge to the conclusion that the execution of various visuospatial tasks consistently activates bilateral frontal and parietal regions in the "visuospatial network" of the brain.

However, research using TMS to disrupt regional neural processing has indicated that only the right, not the left, parietal network is functionally relevant for (visuo)spatial processing (Collignon et al., 2008; Valero-Cabre, Pascual- Leone, \& Rushmore, 2008), visuospatial attention ( Jin \& Hilgetag, 2008; Rounis, Yarrow, \& Rothwell, 2007; Kim et al., 2005; Muri et al., 2002; Hilgetag, Theoret, \& PascualLeone, 2001), visuomotor processing (Bestmann, Thilo, Sauner, Siebner, \& Rothwell, 2002; Rushworth, Ellison, \& Walsh, 2001), visuospatial imagery (Sack et al., 2005), and visuospatial judgment (Sack et al., 2002, 2007).

This apparent right hemispheric dominance for visuo-spatial functions was further elucidated by a recent simultaneous TMS and fMRI study of our group, in which the neural correlates of TMS-induced behavioral impairment on a visuospatial judgment task were assessed "online" (Sack et al., 2007). To this end, we administered TMS over either left or right posterior parietal cortex (PPC), while concurrently measuring both the behavioral as well as neural consequences of parietal TMS using whole-brain fMRI scanning. Only right parietal TMS, not left, induced a behavioral impairment on the visuospatial judgment task, but not on a control task. Simultaneously, only right parietal TMS, not left, resulted in a TMS-induced BOLD signal decrease throughout a right hemispheric fronto-parietal network during visuospatial judgment, but not during 
control task execution. In other words, TMS over right parietal cortex not only reduced brain activity at the site of stimulation, but also in ipsilateral remote frontal regions, including right MFG. Interestingly, the amount of BOLD signal decrease in this network, both in local and remote regions, correlated highly with the amount of TMSinduced visuospatial impairment.

Taken together, all of these findings seem to indicate that a fronto-parietal network in the right hemisphere underlies visuospatial processing. Moreover, as the most recent simultaneous TMS and fMRI study on visuospatial judgment (Sack et al., 2007) suggested, the interactions within this network are such that disruption of processing at one node of the network (i.e., the parietal node) has measurable and comparable neural effects at the other nodes (e.g., within MFG). Still, the exact taskspecific dynamics, temporal characteristics, and direction of influences within this specific right hemispheric fronto-parietal network during the execution of visuospatial judgments remain unknown. In the current study, we used time-resolved, fast eventrelated fMRI in combination with multivariate data-driven functional and effective brain connectivity analysis tools in order to investigate the brain network dynamics underlying visuospatial judgment in the right hemisphere.

For optimal comparability with previous research, we adopted the same behavioral tasks used by Sack et al. (2002, 2007): a visuospatial judgment task (ANGLE) and color judgment control task (COLOR). We specifically evaluated task modulation of functional connectivity within this network, and proceeded to investigate directed influences within this task-specific functionally connected network. We purposely adopted a recently developed exploratory effective connectivity analysis technique (Granger causality mapping [GCM]; see Roebroeck, Formisano, \& Goebel, $2005)$ to avoid restriction of analysis to predefined regions. This way, we were able to explore the interactions within and beyond the conventionally defined visuospatial network in the human right hemisphere.

\section{Materials and Methods}

\section{Participants}

Ten healthy participants ( 5 men, 8 right-handed) were included in this study (mean age $=23.3$ years; $\mathrm{SD}=1.8$ years). All had normal or corrected-to-normal vision, no history of neuropsychiatric disorders, and eight had previously participated in fMRI experiments. The experiment was approved by the local ethical committee, written informed consent was obtained before participation. Participants were screened for fMRI experimentation safety and received monetary compensation.

\section{Stimuli and Task}

Participants were presented with visual stimuli inside the MRI scanner. Each stimulus was projected for $300 \mathrm{msec}$ at center fixation. Participants were asked to fixate at all times, aided by a grey fixation cross between stimuli. The stimuli consisted of 
schematized analogue clocks with yellow rims and two either white or yellow hands (13/33 yellow). The hands of the clocks formed different angles, categorized as small or large (13/33 small). All stimuli and fixation crosses were taken from our previous study (Sack et al., 2007). Participants were asked to press one of two buttons per stimulus, depending on whether the stimulus was a target (right index finger response) or a nontarget (right middle finger response). In the ANGLE task, clocks with small angles $\left(30^{\circ}\right.$ or $60^{\circ}$ angles between the hands) were targets, clocks with large angles (bigger than $60^{\circ}$ ) were nontargets. In the COLOR task, clocks with yellow hands were targets, clocks with white hands were nontargets (see Figure 1 for an illustration of stimuli and tasks). Stimuli were presented, and response times recorded, using Presentation Software (Neurobehavioral Systems, San Francisco, CA). Response speed and accuracy were equally emphasized in instructions to the participants.

The experimental design was mixed; blocks of stimuli were presented in a rapid event-related design. One block contained 11 trials (10 task trials: 4 targets and 6 nontargets, and one null trial). Throughout a block, the task was constant. The task for each block was made known to the participant prior to the block, in the form of a oneletter cue: "A" for ANGLE, "C" for COLOR. The order of blocks was pseudorandomized, as was the order of trials within the blocks. A total of 28 blocks (=280 task trials), divided equally over two fMRI functional runs, was presented. Within blocks, the intertrial interval was jittered around 3000 to $4500 \mathrm{msec}$. Time between blocks was $7500 \mathrm{msec}$, including the $2000 \mathrm{msec}$ task instruction.

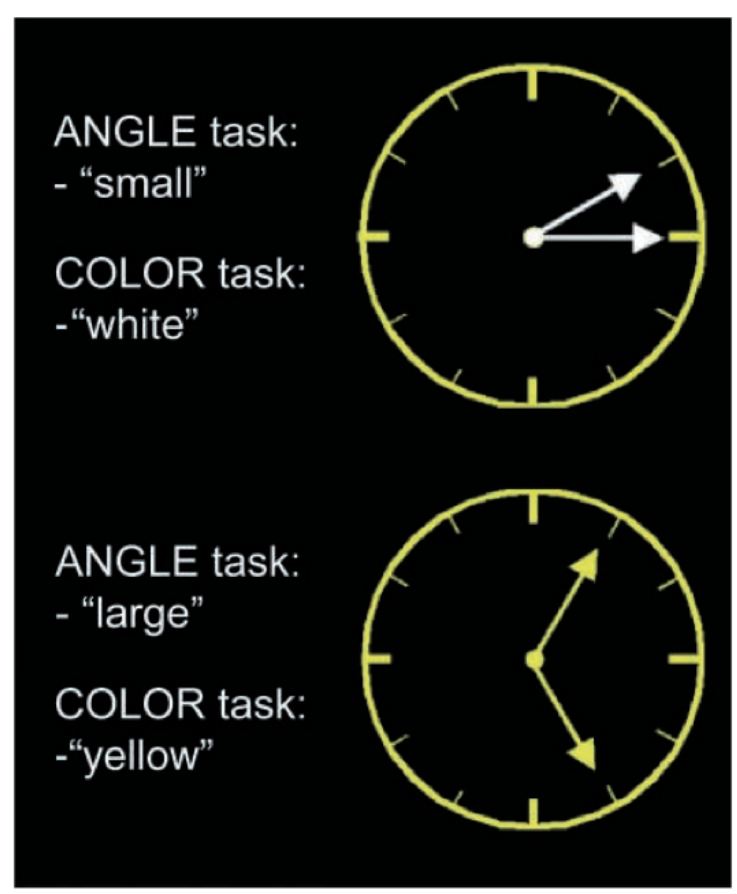

Figure 1. Stimuli and tasks. Two example stimuli are shown with appropriate responses for each task. The same stimuli were used in both the ANGLE and the COLOR tasks. In the ANGLE task, the two response options corresponded to a "small" angle $\left(30^{\circ}\right.$ or $\left.60^{\circ}\right)$ and a "large" angle (more than $60^{\circ}$ ) between the two clock-hands. In the COLOR task, the two response options reflected the two possible clock-hands colors: "yellow" and "white." 


\section{MRI Parameters and Functional Data Processing}

MRI was performed using a 3-Tesla Siemens Allegra scanner (Siemens, Erlangen, Germany). A standard transmit- receive head coil was used to obtain high-resolution anatomical [ADNI, T1-weighted, flip angle $(\mathrm{FA})=9^{\circ}, \mathrm{TR}=2250, \mathrm{TE}=2.6 \mathrm{msec}, 192$ slices, field of view $(\mathrm{FoV})=256 \mathrm{~mm}$, isotropic voxel resolution of $\left.1 \times 1 \times 1 \mathrm{~mm}^{3}\right]$ and whole-brain functional ( $\mathrm{T} 2 *$-weighted echo-planar imaging; $\mathrm{FA}=60^{\circ}, \mathrm{TR}=1500$, TE $=28 \mathrm{msec}$, acquisition gap $=500 \mathrm{msec}, 18$ oblique contiguous slices, slice thickness $=5$ $\mathrm{mm}, \mathrm{FoV}=224 \mathrm{~mm}, 64 \times 64$ voxel matrix, voxel resolution $=3.5 \times 3.5 \times 5 \mathrm{~mm}^{3}$ ) images. Participants' hearing was protected using earplugs and headphones. Head movement was restricted using foam pads.

FMRI data were processed using BrainVoyager QX (Brain Innovation, Maastricht, the Netherlands). Preprocessing included inter-scan slice acquisition time correction, linear trend removal, temporal high-pass filtering to remove low- frequency drifts, and rigid-body transformation of data to the first acquired image to correct for motion. Please note that functional data were not smoothed spatially; reported data were unsmoothed throughout all analyses. Functional data were coregistered to anatomical data per subject, and subsequently transformed to Talairach space (Talairach and Tournoux, 1988) or aligned to a dynamic group average brain by means of cortex-based alignment (CBA).

\section{Cortex-based Alignment}

For individual right hemispheres, the grey-white matter boundary was determined to segment and reconstruct the cortical surface (Kriegeskorte \& Goebel, 2001). Functional data in volume space were sampled to surface vertices in a direction perpendicular to the gray-white matter boundary, and thus, converted to surface space. The idiosyncratic folding patterns of sulci and gyri were mapped in a spherical coordinate system. In this common space, a reiterative process morphed the individual hemispheric surfaces to a dynamic group average. This resulted in an average group brain. Because functional data were mapped to surface space, the surface-to-sphere and sphere-to-group average transformation mappings also afforded group alignment of functional data. We applied this process of CBA to our general linear model (GLM) contrast analyses and GCM connectivity analyses because it has been shown to yield superior statistical power (Fischl, Sereno, Tootell, \& Dale, 1999).

\section{Activation Data Analysis}

For functional data analysis, BOLD time courses of individual vertices were regressed onto a prespecified model in a conventional GLM. Predictors were based on $300 \mathrm{msec}$ events convolved with a hemodynamic response gamma function (Boynton, Engel, Glover, \& Heeger, 1996). Separate predictors were implemented for ANGLE clock presentations, COLOR clock presentations, and INSTRUCTIONS ("A" and " $C$ " conjoined in one model predictor). Effects of interest included activation during the ANGLE or the COLOR task contrasted with baseline activity and activation during the 
ANGLE versus the COLOR conditions as contrasted directly in the GLM. Activation differences on the group level were analyzed directly on the vertex level, leading to statistical parametric maps on a group-average brain [an additional patch-of-interest (POI) analysis is presented and discussed on-line: www.tomdegraaf.com]. Corrections for multiple comparisons were made using cluster threshold level estimation (Hagler, Saygin, \& Sereno, 2006; Forman et al., 1995), with 1000 iterations of Monte Carlo simulation setting a statistical threshold of $p<.01$ for the main task effects and $p<.05$ for the ANGLE $>$ COLOR contrast. To ensure generalizability of the results, all analyses reported were performed on a random effects (RFX) level (unless indicated otherwise). BOLD time courses for regions of interest (ROIs) were approximated by means of event-related deconvolution analysis. This method can be applied in rapid event-related designs to extract the BOLD response to single events from the overlapping pattern of activation. Activation is regressed on separate "stick" predictors per scanning time point. This results in one beta value per TR in the volumes following an event. These beta values reflect the BOLD response pattern to an event.

\section{Functional and Effective Connectivity Analysis}

Functional connectivity has been defined as correlation between remote neurophysiological events in the temporal domain (e.g. Friston, 1994). Functional connectivity analysis can reveal networks of connected regions that show similar activity changes during mental task performance. Thus, in our case, functional connectivity refers to task-specific instantaneous correlation of BOLD time courses during task execution. Presumably, remote regions with BOLD time courses that correlate during visuospatial judgment task execution, but not execution of a control task, are working together on the mental processes involved in visuospatial judgment. Effective connectivity has been defined as directed influence from one region to another, and allows one to move toward causal inferences (Friston, 1994). More precisely, we examined task-specific effective connectivity to ROIs throughout the brain. To this end, we applied GCM (Roebroeck et al., 2005). GCM requires one to specify a seed region after which measures of (functional or effective) connectivity for all voxels or vertices in the brain are calculated, in reference to the time course in the seeded cluster. The maps obtained illustrate which areas in the brain are functionally connected to the seed region (functional connectivity maps), or which areas in the brain send influence to or receive influence from the seed region (effective connectivity maps).

GCM works on two time series, $\mathrm{X}[\mathrm{t}]$ and $\mathrm{Y}[\mathrm{t}]$ (activity over time in Region $\mathrm{X}$ and Region $\mathrm{Y}$ ). The precise meaning of found connectivity between $\mathrm{X}$ and $\mathrm{Y}$ is a decrease of uncertainty about time points of $Y$, due to knowledge about past time points of X. Simply put, knowing one time course tells you something extra about the other time course. The direction of influence is based on which time course lags behind the other. Thus, if knowing $\mathrm{X}[\mathrm{t}-1]$ (history of BOLD in Region $\mathrm{X}$ ) improves the prediction of $\mathrm{Y}[\mathrm{t}]$ (BOLD time course of Region $\mathrm{Y}$ ) over and above the prediction achieved using $\mathrm{Y}$ [t-1] (Region Y's own BOLD history), then the activity in Region $\mathrm{X}$ is said to Granger cause the activity in Region Y. By regressing one time series on both itself and 
another, influences can be inferred with suitable models (vector autoregressive models). Directionality is thus concluded on the basis of temporal precedence. All effective connectivity maps were restricted to vertices that also had high instantaneous correlations to minimize effects of draining veins (for further details, see Roebroeck et al., 2005).

GCM has successfully been applied in previous studies (e.g. Bien, Roebroeck, Goebel, \& Sack, 2009; Sack et al., 2008; Sack et al., 2007; Abler et al., 2006). However, these studies only looked at individual participants or mean GCM maps, or performed fixed effects GCM analysis. The current study explicitly evaluated statistically thresholded RFX functional and effective connectivity, by calculating RFX GCM maps as follows: (1) GCM maps were calculated for each individual participant in surface space (on the reconstructed cortical surface with functional data projected onto it), or volume space. Statistical testing for individual maps was based on bootstrap simulations (see Roebroeck et al., 2005), with corrections for multiple comparisons based on false discovery rate $(\mathrm{q}<0.05)$ (Genovese, Lazar, \& Nichols, 2002). (2) In surface space, these individual maps were cortex-based aligned, and second-level t tests (per vertex, over subjects) were calculated. Correction for multiple comparisons in these RFX group maps was based on cluster-size threshold estimation (1000 iterations; see Hagler et al., 2006; Forman et al., 1995).

To analyze connectivity in individual participants (and thus create the aforementioned individual GCM maps which were subsequently overlaid to obtain RFX group maps), we determined a POI to seed into the GCM procedure as follows: A GLM conjunction analysis of ANGLE versus baseline and COLOR versus baseline [(A $>B)$ $\&(\mathrm{C}>\mathrm{B})]$ was performed, revealing regions where activity was modulated by both tasks. From these regions, the cluster in PPC with the highest difference in activity between ANGLE and COLOR (with activity higher during ANGLE trials) was determined. This region was assumed to be engaged in visuospatial processing and served as the starting point for GCM analysis of the visuospatial network. Post-hoc confirmatory GCM analyses were performed by seeding into the GCM procedure new seed regions defined on the basis of individual GCM maps or group RFX GCM maps.

To summarize, for each individual participant, task-specific functional connectivity maps were created based on instantaneous correlation of vertices throughout the entire brain, to the seed region. Effective connectivity per experimental condition was calculated in step 2 for each individual participant. In step 3, the individual maps were cortex-based aligned (for the surface space analyses) and overlaid. This created a RFX group GCM map projected onto the average group brain. Statistical significance of the results was tested as described above.

\section{Results}

\section{Behavioral Results}

Reaction times and accuracy were $675.4 \mathrm{msec}(\mathrm{SD}=166.0)$ and $6.3 \%$ incorrect for ANGLE, and $650.5 \mathrm{msec}(\mathrm{SD}=175.5)$ and $9.7 \%$ incorrect for COLOR, respectively. 
Sack et al. (2007) used the same task but found faster average reaction times: $495 \mathrm{msec}$ and $452 \mathrm{msec}$ for ANGLE and COLOR tasks, respectively. But these authors used a block design with regular and predictable stimulus onsets. A brief follow-up behavioral experiment revealed that five new participants were, on average, $156.3 \mathrm{msec}$ quicker to respond in a block design as applied by Sack et al. (2007) than in the mixed design implemented in the current study, confirming experimental design to be responsible for the differences.

\section{Brain Activation Results}

We performed a RFX GLM analysis on the vertex time courses in cortex-based aligned surface space (see Methods). Figure 2A shows thresholded RFX activation maps, on the cortex-based aligned group-average inflated brain, for ANGLE versus baseline and for COLOR versus baseline separately $[\mathrm{t}(9)=3.25, \mathrm{p}<.01$, corrected]. An extensive fronto-parietal activation network is revealed, notably including middle/inferior frontal gyrus (MFG) and PPC. The medial views of the reconstructed right hemisphere reveal activation in supplementary motor area and large regions of the occipital lobe, in both tasks. The lateral views show that regions of prefrontal cortex, occipital- temporal cortex (OT), and the insula (INS) are activated in both tasks.

To examine which of these activations were privy to the visuospatial network, we contrasted the activity in the ANGLE and COLOR conditions in a RFX GLM. In surface space, after CBA, a RFX GLM on these data revealed two regions to be significantly more active in response to ANGLE executions as compared to COLOR executions (i.e., PPC and MFG), and three regions more active during COLOR as compared to ANGLE [i.e., SMG, an anterior region of MFG (aMFG), and SFG; $\mathrm{t}(9)=$ $2.262, \mathrm{p}<.05$, corrected]. Figure $2 \mathrm{~B}$ visualizes these regions in a contrast map (for coordinates and more information on these and other task-specific areas, see www.tomdegraaf.com).

\section{Functional Connectivity during Visuospatial Processing}

Before investigating directionality of influences in the visuospatial network, we examined functional connectivity (instantaneous correlation) within this network during the visuospatial ANGLE task and the COLOR control task. Co-activation of regions during task processing does not guarantee a functional connection, let alone taskspecific functional connectivity (instantaneous correlations in regional time courses during one task but not another). However, we were able to demonstrate that PPC engaged in significantly stronger functional connectivity to MFG during visuospatial processing than during our control task. Thus, this fronto-parietal network communicated more vigorously during visuospatial judgment. 


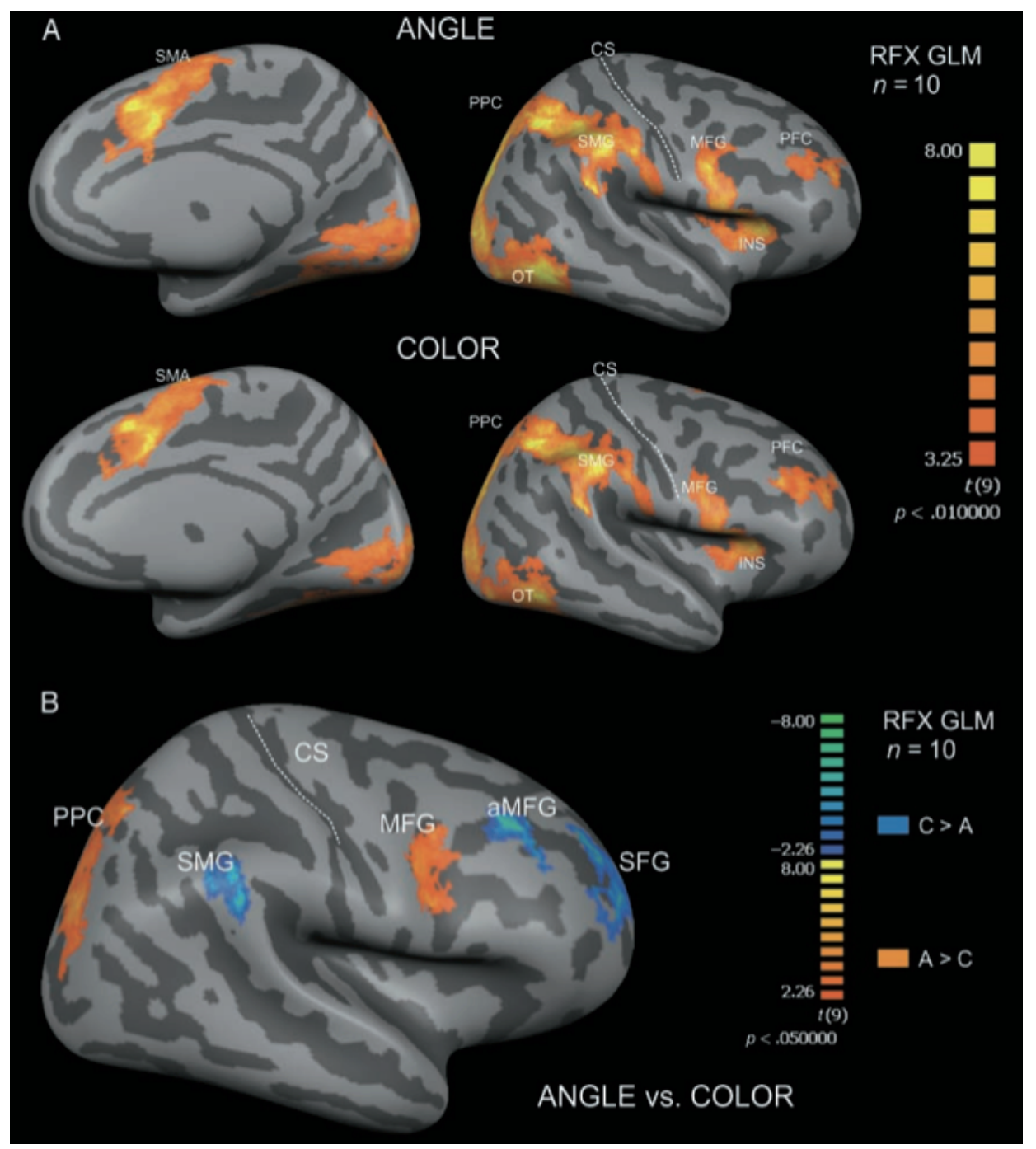

Figure 2. RFX activation maps.

(A) Cortex-based aligned RFX GLM for ANGLE and COLOR conditions. Vertices significantly more active during task processing than baseline are shown in orange-yellow $[\mathrm{t}(9)=3.25, \mathrm{p}<.01$, corrected]. (B) A cortex-based aligned RFX GLM contrast map. Vertices more active during ANGLE are shown in orange-yellow, vertices more active during COLOR are shown in blue-green $[\mathrm{t}(9)=2.262, \mathrm{p}<.05$, corrected].

To visualize the spread of seed regions across participants, Figure 3 shows in varying colors the MFG POIs of all 10 participants (after CBA) seeded into GCM. We computed a RFX group map of task modulation of connectivity, in cortex-based aligned surface space. Figure 3 thus shows which regions were significantly $[\mathrm{t}(9)=2.262$, $\mathrm{p}<$ .05 , corrected] more strongly connected to MFG during ANGLE blocks than during COLOR blocks. PPC is more connected to MFG, as are parts of parieto-occipital 
cortex, during ANGLE as compared to COLOR pseudoblocks. Thus, this map visualizes statistically significant task modulation of functional connectivity within the visuospatial network. Task modulation is important and informative; it ensures that the results reveal functional brain organization that is task-related. Having established task modulation of the connectivity within the network, we also investigated directionality of influences within this network.

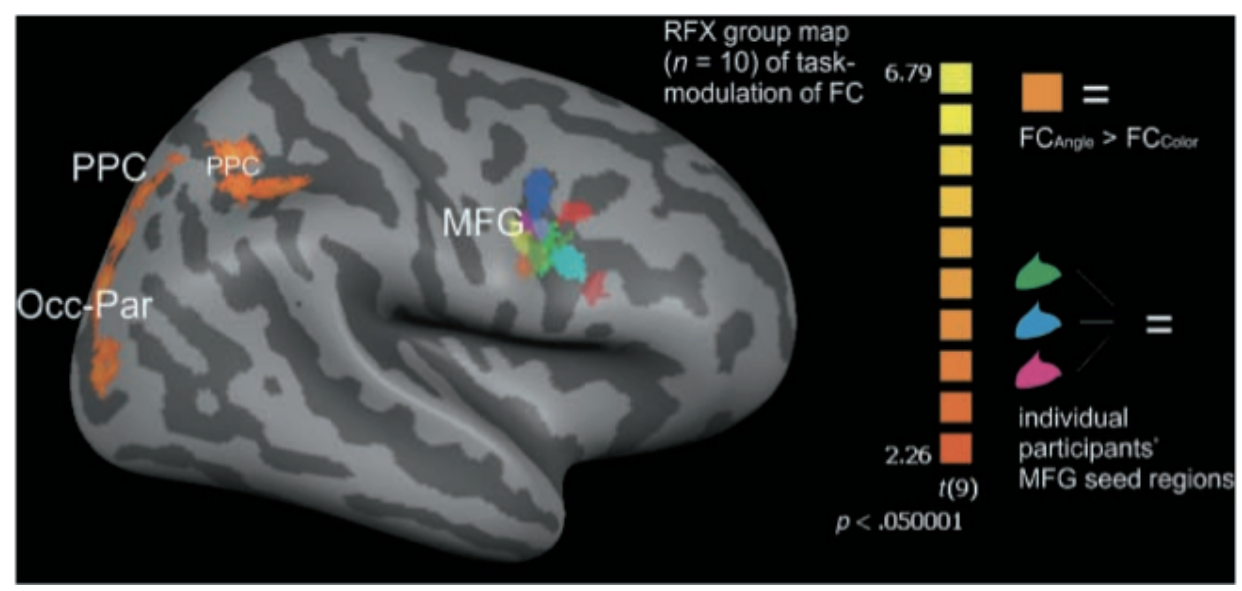

Figure 3. RFX connectivity difference map.

RFX group contrast based on cortex-based aligned functional and anatomical data. This map reveals task modulation of instantaneous functional connectivity between MFG and other parts of the brain. Vertices that are significantly more functionally connected (FC) to MFG during the visuospatial ANGLE task than during the COLOR control task are shown in orange-yellow $[\mathrm{t}(9)=2.262, \mathrm{p}<.05$, corrected]. Thus, $\mathrm{PPC}$ is more connected to MFG during ANGLE than during COLOR. This task modulation of connectivity indicates that the fronto-parietal network operates more as a whole during ANGLE conditions than during COLOR conditions. This group map was calculated after individual functional connectivity maps were obtained, based on individual seed POIs. To illustrate inter-participant spread of these seed regions, the individual MFG POIs on which the analyses were based are shown in varying colors, after cortex-based alignment of the patches.

\section{Effective Connectivity during Visuospatial Processing}

RFX GCM effective connectivity analysis, referenced to PPC, revealed a frontal-toparietal direction of influence. Figure 4 shows statistically significant $[\mathrm{t}(9)=2.262, \mathrm{p}<$ .05 , corrected] influences to and from the seed region as revealed by GCM, for ANGLE and COLOR separately. Green areas influence (project to) the PPC seed region (red), whereas blue areas are influenced by PPC. During ANGLE, MFG, a second parietal cluster, and INS all significantly projected to the PPC seed region $[\mathrm{t}(9)=2.262, \mathrm{p}<.05$, corrected]. During COLOR, no statistically significant influences were found. Please note, however, that this lack of statistical significance does not imply that there was absolutely no effective connectivity between PPC and MFG during the COLOR task, 
but that it was weaker in comparison to the effective connectivity during the ANGLE task and, unlike during ANGLE, not statistically significant (for further illustration of locations and extent of task modulation of effective connectivity, see S2 on www.tomdegraaf.com).

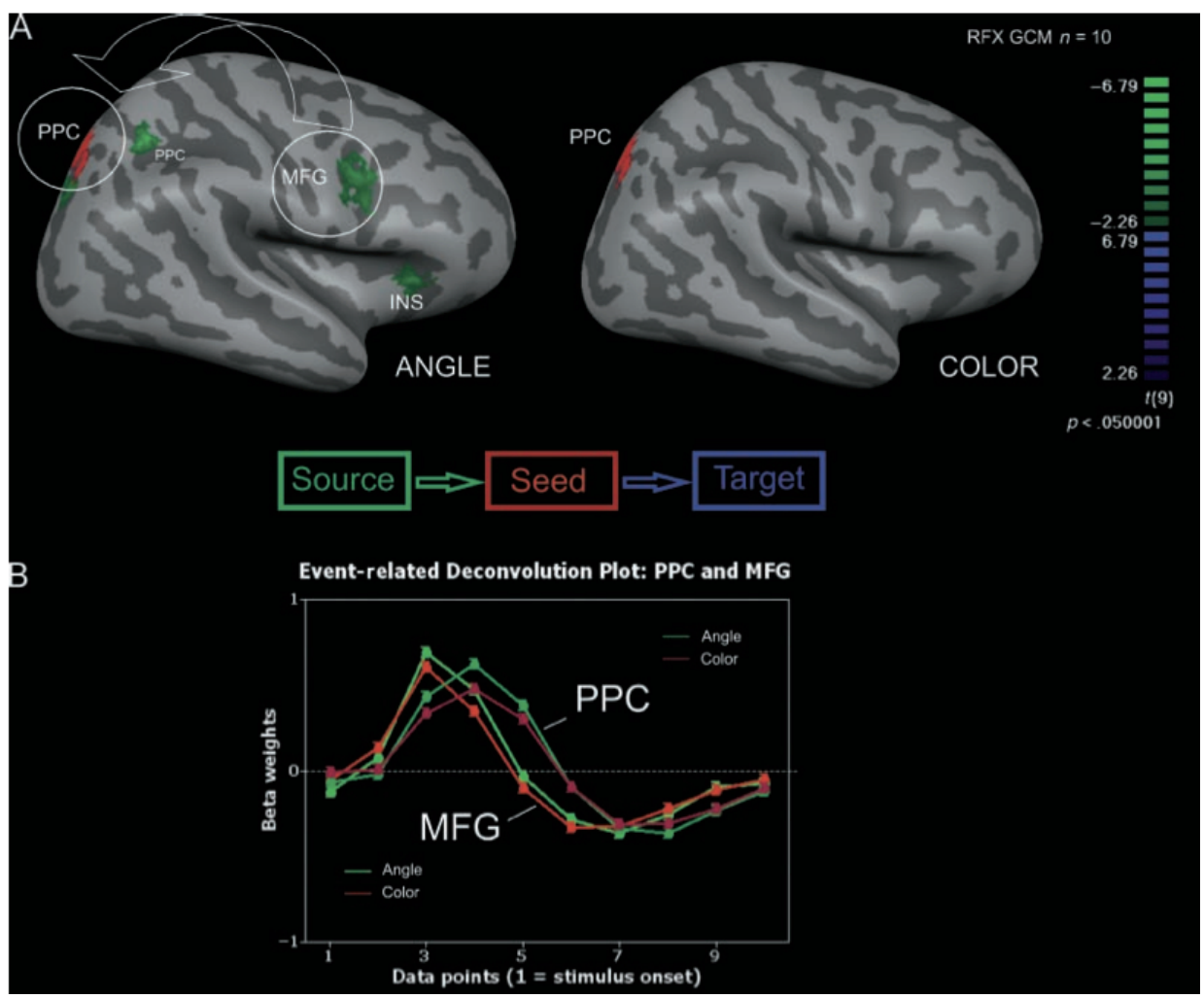

Figure 4. Task-specific effective connectivity.

(A) GCMs, showing task-specific directed influences throughout the entire brain, referenced to seed region PPC (in red). Shown here are statistically significant projections [ $\mathrm{t}(9)=2.262$, $\mathrm{p}<.05$, corrected] to PPC from MFG, INS, and a second parietal cluster, during the ANGLE task specifically. No significant connectivity to PPC was found for the COLOR condition.

(B) Event-related deconvolution plots approximate the regional BOLD time courses for PPC and MFG per task. This plot implies earlier involvement of MFG than PPC.

A post-hoc analysis with seed region MFG confirmed that MFG did significantly project to PPC during ANGLE, but also significantly projected to OT during ANGLE and not during COLOR $[\mathrm{t}(9)=2.262, \mathrm{p}<.05$, corrected; data not shown]. For PPC and MFG, event-related deconvolution plots approximate the regional time courses. Note that different latencies of the average (deconvolved) BOLD response are not equivalent to GCM directed influences (see Roebroeck et al., 2005, for details). However, in this case, the BOLD time-course plots clearly confirm earlier involvement of MFG than PPC during both tasks, with stronger activation for ANGLE in both regions (see Figure 4). 
For several other regions, there were indications of effective connectivity to PPC. Postcentral gyrus (PCG), occipital cortex (OT), and superior frontal sulcus (SFS) were effectively connected to PPC in individual GCM maps, mean GCM group maps, and in the more conservative RFX GCM group analysis, these regions showed a statistical tendency toward PPC during ANGLE $[\mathrm{t}(9)=1.83, \mathrm{p}<.1$, corrected] but not during COLOR [with the exception of PCG; $\mathrm{t}(9)=1.83, \mathrm{p}<.1$ ]. Interestingly, SFS showed a particularly strong task modulation of effective connectivity (on par with connectivity modulation in MFG): SFS interacted much more with PPC during ANGLE than during COLOR (see S2 on www.tomdegraaf.com).

To summarize, MFG and INS significantly influenced PPC during ANGLE, but not during COLOR, as indicated by effective connectivity analysis referenced to PPC. Furthermore, there were indications that PCG, OT, and SFS may also engage in network interactions during visuospatial processing.

We also identified COLOR-specific regions; aMFG, SFG, and SMG. However, RFX GCM analysis to these regions yielded no significant effective connectivity to these regions in either the ANGLE or the COLOR task, other than vertices or small clusters surrounding the seed region, with the exception of SFG in the ANGLE task, where SFG received influence from INS $[\mathrm{t}(9)=2.262, \mathrm{p}<.05$, corrected; data not shown].

\section{Discussion}

Our data replicate earlier studies on visuospatial processing by revealing involvement of frontal and parietal regions during visuospatial judgment. However, our results go beyond previous research by demonstrating that these regions were indeed operating as a network during execution of our task. Thus, we revealed statistically significant task modulation of functional connectivity within the visuo-spatial network (i.e., between PPC and MFG). Furthermore, GCM allowed us to investigate directional influences within this network, revealing a frontal-to-parietal information flow.

\section{New Brain Activation Findings}

Our main ANGLE-specific activations, in MFG and PPC, mirror an established body of work on visuospatial cognition (see Introduction). However, in previous research using the exact same stimuli and tasks, our COLOR-specific regions were not consistently identified (Sack et al., 2002, 2007). This may be attributed to several methodological differences between our and previous work that could have lead to respective differences in statistical sensitivity for revealing these COLOR-specific brain regions. First, we applied new procedures of data analysis: Our study involved CBA, GLM analysis in surface space, and no spatial smoothing. Second, whereas the previous work with our stimuli and tasks involved block designs, we employed a rapid event-related design. This resulted in more frequent changes between tasks and in unpredictable delays between trials. As indicated in the Results section and confirmed in a behavioral pilot experiment, the new design also resulted in different behavioral results. It is difficult to disentangle these factors. Unfortunately, we gained no further insights from 
GCM analysis as almost no significant effective connectivity was found to these regions in both tasks. However, the fact that our conservative GCM analysis did not produce miscellaneous influences to these regions further validates the results we did find for the experimental task. The remainder of the discussion will focus on these interactions underlying execution of the ANGLE task.

\section{Fronto-parietal Information Flow}

A primary aim of our study was to examine the nature of interactions between MFG and PPC during the execution of visuospatial judgment. Interestingly, our results revealed information flow to be mainly from MFG and other frontal regions, including INS and possibly SFS, PCG, and OT, toward PPC. This dominant fronto-parietal direction is noteworthy, because a classical view of visuospatial processing involves a hierarchical dorsal striate-parieto-frontal stream. Dominant MFG-to-PPC projections seem to go against this stream (mirroring the conceptions of recurrent processing being fundamental to visual processing as discussed in Part II of this dissertation).

One explanation of this direction of influence stems from the nature of our cognitive task. Visuospatial judgment is, by definition, goal-related: A judgment involves options, choices, and, in our case, a template to match input to [the "target angle" is the angle between clock- hands that defines the border between Response 1 (small angle) and Response 2 (large angle)].

We propose that MFG was instrumental in a process of stored template matching, biasing the processing of visuospatial features in PPC using top-down signals, in light of the task-relevant comparison with the target angle template. Right MFG has been implicated in various higher-order central executive processes, such as selection, monitoring, and organization of processing (e.g., Curtis \& D'Esposito, 2003; Rypma \& D'Esposito, 2003; Collette \& Van der Linden, 2002); all forms of top-down (attention) control (see Yeh, Kuo, \& Liu, 2007). Already some years ago, ventral lateral prefrontal cortex was proposed to serve as an early recipient of information from posterior parietal regions and as the site where comparisons are made with representations in working memory (D'Esposito et al., 1998; Owen, Evans \& Petrides, 1996; Petrides, 1994). More recently, directed influences from right MFG to right superior parietal cortex have been found in a flanker task with a spatial twist and in an fMRI study using structural equation modeling to study effective connectivity (Erickson, Ringo Ho, Colcombe \& Kramer, 2005). This information flow was interpreted in terms of attentional set (based on, for example, Banich et al., 2000).

Moreover, it has been suggested that neural processing in lower areas is most efficient under the direction of, or in interaction with, top-down signals from a higherorder region (Kveraga, Ghuman \& Bar, 2007). In sustained attention tasks (which our tasks seem to be), it has been suggested specifically that right medial frontal regions influence, top-down, posterior cortex to bias and enhance processing (e.g., see Sarter, Givens \& Bruno, 2001, for a review). Taken together, this literature leads us to speculate that the MFG-to-PPC projections were focusing the processing in PPC on the relevant visuospatial features, either prior to stimulus onset to sensitize the relevant 
neurons and/or immediately upon stimulus presentation to bias processing toward the relevant visuospatial information.

Methodological limitations constrain the extent of our interpretation. Unfortunately, bidirectional influences between two regions are lost in GCM maps. Because the effective connectivity values are based on a difference measure of the influence in two opposite directions (for details, see Roebroeck et al., 2005), the results reflect the dominant influence only. Thus, a unidirectional MFG-to-PPC influence identified by GCM does not preclude a communication flow in the reverse direction per se. It just means that, overall, MFG predominantly influenced PPC during ANGLE pseudoblocks. The interpretation of bidirectional, but asymmetrical, fronto-parietal flow is consistent with research by Edin, Klingberg, Stodberg, and Tegner (2007). These authors integrated EEG, fMRI, and neurocomputational modeling to show that, in a visuospatial working memory task, a bidirectional fronto-parietal model with stronger frontal-to-parietal (i.e., SFS-to-IPS) connections than parietal-to-frontal (i.e., IPS-toSFS) connections best explained the data. Indeed, in their study, stronger SFS-to-IPS effective connectivity was shown to protect against distracters on visuospatial working memory performance. Thus, fronto-parietal asymmetry was functionally relevant. Apparently, this may be a mechanism that is not specific to our task.

Because GCM is based directly on BOLD signals rather than neural signals, intrinsic hemodynamic response differences between brain areas could confound GCM results of a given condition. This is countered by using a control condition (in our case, COLOR) to ensure task specificity of the connectivity results. Our MFG-to-PPC influence was significant only in the visuospatial task and not in the control task. Another limitation of GCM is that it remains uncertain whether information flows directly from an identified region to the seed region, or via other brain areas. In light of this, it becomes important to take into account connectivity results throughout the brain.

\section{Functional Roles of Visuospatial Network Nodes}

PPC seems to be involved in many spatial processes, as has been discussed in the Introduction. It is therefore plausible to assume that the analysis of the spatial features of our stimuli was centered in PPC. Lehmann, Vannini, Wahlund, Almkvist, and Dierks (2006) used task demand, as measured by RT, as a predictor in GLM analysis. Their cognitive task was highly similar to the one used in this study; involving judgment of clock-hands. PPC, a region near MFG, as well as INS, all revealed BOLD signal correlation to task demand: strong evidence for direct visuospatial task involvement of all these regions.

Both SFS and MFG have been implicated in spatial working memory tasks. The role of SFS in spatial working memory seems well established (Zarahn, Aguirre, \& D’Esposito, 1999; Courtney, Petit, Maisog, Ungerleider, \& Haxby, 1998; Petit et al., 1998). To properly perform our task, a representation of the "target angle" must have been maintained in order to serve as template to match new visuospatial input to. The SFS may have stored that template. Sack et al. (2008) instructed participants to imagine a certain visuospatial object, which was rotated compared to a remembered target object. Participants were then asked to determine whether the two objects were the 
same. Using GCM, Sack et al. found a premotor-to-parietal direction of influence underlying this task. On inspection, their premotor area may correspond to our SFS. To actually perform the stored template matching, MFG could come into play, as detailed above. This proposed mechanism is in line with previous research. Glahn et al. (2002) showed involvement of both MFG and SFS in a spatial working memory task, but were able to dissociate the contributions of both regions. SFS was involved specifically in maintenance of spatial working memory contents, whereas MFG seemed to be involved specifically in spatial manipulation of these contents (see also Postle et al., 2000).

Ventral area OT may engage in the recognition and formation of the clock object, which our stimuli comprised. This object information was likely more relevant to the processing of an angle between real object parts: the clock-hands, than a basic property such as color, which is observed at a glance. The involvement of OT in object processing is relatively established (e.g., Grill-Spector, 2003).

Putting our results together with this literature, we thus speculate that visuospatial judgement in our paradigm involved the following neurocognitive steps. Information from occipital cortex will proceed along the ventral and dorsal stream to frontal areas. Dorsal frontal area SFS might maintain the template of the angle that distinguished Response 1 ("small angle") from Response 2 ("large angle"). As detailed above, the MFG can use this representation to modulate PPC by means of top-down signals. Frontal region MFG thus "focuses" the PPC processing activities to the proper parts of the stimulus, pre-sensitizing neural activity in PPC prior to stimulus onset and/or biasing neural activity after stimulus onset. PPC could then analyze the spatial features, MFG orchestrate which spatial features are task-relevant (on the basis of the defining visuospatial template held in SFS working memory), and OT pitching in possibly relevant object information. Such distributed processing across the brain clearly would require extensive communication, which might partly have been revealed by our data. Note that such interpretations necessarily remain speculative. Moreover, the model is clearly incomplete. Nevertheless, the proposed functional segregations within our network are compatible with the data as presented here and by others, and may provide fruitful new hypotheses for further research. 


\section{References}

Abler, B., Roebroeck, A., Goebel, R., Hose, A., Schonfeldt- Lecuona, C., Hole, G., et al. (2006). Investigating directed influences between activated brain areas in a motorresponse task using fMRI. Magnetic Resonance Imaging, 24, 181-185.

Banich, M. T., Milham, M. P., Atchley, R. A., Cohen, N. J., Webb, A., Wszalek, T., et al. (2000). Prefrontal regions play a predominant role in imposing an attentional "set": Evidence from fMRI. Brain Research, Cognitive Brain Research, 10, 1-9.

Bestmann, S., Thilo, K. V., Sauner, D., Siebner, H. R., \& Rothwell, J. C. (2002). Parietal magnetic stimulation delays visuomotor mental rotation at increased processing demands. Neuroimage, 17, 1512-1520.

Bien, N., Roebroeck, A., Goebel, R., \& Sack, A. T. (2009). The brainðs intention to imitate: The neurobiology of intentional versus automatic imitation. Cerebral Cortex, 19, 2338-2351.

Boynton, G. M., Engel, S. A., Glover, G. H., \& Heeger, D. J. (1996). Linear systems analysis of functional magnetic resonance imaging in human V1.

Cohen, M. S., Kosslyn, S. M., Breiter, H. C., DiGirolamo, G. J., Thompson, W. L., Anderson, A. K., et al. (1996). Changes in cortical activity during mental rotation. A mapping study using functional MRI. Brain, 119, 89-100.

Collette, F., \& Van der Linden, M. (2002). Brain imaging of the central executive component of working memory. Neuroscience and Biobehavioral Reviews, 26, 105125.

Collignon, O., Davare, M., De Volder, A. G., Poirier, C., Olivier, E., \& Veraart, C. (2008). Time-course of posterior parietal and occipital cortex contribution to sound localization. Journal of Cognitive Neuroscience, 20, 1454-1463.

Corbetta, M., Kincade, J. M., \& Shulman, G. L. (2002). Neural systems for visual orienting and their relationships to spatial working memory. Journal of Cognitive Neuroscience, 14, 508-523.

Courtney, S. M., Petit, L., Maisog, J. M., Ungerleider, L. G., \& Haxby, J. V. (1998). An area specialized for spatial working memory in human frontal cortex. Science, 279, 1347-1351.

Culham, J. C., Cavina-Pratesi, C., \& Singhal, A. (2006). The role of parietal cortex in visuomotor control: What have we learned from neuroimaging? Neuropsychologia, 44, 2668-2684.

Curtis, C. E., \& DðEsposito, M. (2003). Persistent activity in the prefrontal cortex during working memory. Trends in Cognitive Sciences, 7, 415-423.

de Lange, F. P., Hagoort, P., \& Toni, I. (2005). Neural topography and content of movement representations. Journal of Cognitive Neuroscience, 17, 97-112.

D’Esposito, M., Aguirre, G. K., Zarahn, E., Ballard, D., Shin, R. K., \& Lease, J. (1998). Functional MRI studies of spatial and nonspatial working memory. Brain Research, Cognitive Brain Research, 7, 1-13.

Edin, F., Klingberg, T., Stodberg, T., \& Tegner, J. (2007). Fronto-parietal connection asymmetry regulates working memory distractibility. Journal of Integrative Neuroscience, 6, 567-596. 
Erickson, K. I., Ringo Ho, M. H., Colcombe, S. J., \& Kramer, A. F. (2005). A structural equation modeling analysis of attentional control: An event-related fMRI study. Brain Research, Cognitive Brain Research, 22, 349-357.

Fischl, B., Sereno, M. I., Tootell, R. B., \& Dale, A. M. (1999). High-resolution intersubject averaging and a coordinate system for the cortical surface. Human Brain Mapping, 8, 272-284.

Forman, S. D., Cohen, J. D., Fitzgerald, M., Eddy, W. F., Mintun, M. A., \& Noll, D. C. (1995). Improved assessment of significant activation in functional magnetic resonance imaging (fMRI): Use of a cluster-size threshold. Magnetic Resonance in Medicine, 33, 636-647.

Formisano, E., Linden, D. E., Di Salle, F., Trojano, L., Esposito, F., Sack, A. T., et al. (2002). Tracking the mind's image in the brain: I. Time-resolved fMRI during visuospatial mental imagery. Neuron, 35, 185-194.

Friston, K. (1994). Functional and effective connectivity in neuroimaging: A synthesis. Human Brain Mapping, 2, 56-78.

Genovese, C. R., Lazar, N. A., \& Nichols, T. (2002). Thresholding of statistical maps in functional neuroimaging using the false discovery rate. Neuroimage, 15, 870-878.

Giessing, C., Fink, G. R., Rosler, F., \& Thiel, C. M. (2007). fMRI

data predict individual differences of behavioral effects of nicotine: A partial least square analysis. Journal of Cognitive Neuroscience, 19, 658-670.

Glahn, D. C., Kim, J., Cohen, M. S., Poutanen, V. P., Therman, S., Bava, S., et al. (2002). Maintenance and manipulation in spatial working memory: Dissociations in the prefrontal cortex. Neuroimage, 17, 201-213.

Goebel, R., Linden, D. E., Lanfermann, H., Zanella, F. E., \& Singer, W. (1998). Functional imaging of mirror and inverse reading reveals separate coactivated networks for oculomotion and spatial transformations. NeuroReport, 9, 713-719.

de Graaf et al. 2025

Grill-Spector, K. (2003). The neural basis of object perception. Current Opinion in Neurobiology, 13, 159-166.

Hagler, D. J., Jr., Saygin, A. P., \& Sereno, M. I. (2006). Smoothing and cluster thresholding for cortical surface-based group analysis of fMRI data. Neuroimage, 33, 1093-1103.

Haxby, J. V., Grady, C. L., Horwitz, B., Ungerleider, L. G., Mishkin, M., Carson, R. E., et al. (1991). Dissociation of object and spatial visual processing pathways in human extrastriate cortex. PNAS, 88, 1621-1625.

Hilgetag, C. C., Theoret, H., \& Pascual-Leone, A. (2001). Enhanced visual spatial attention ipsilateral to rTMS-induced "virtual lesions" of human parietal cortex. Nature Neuroscience, 4, 953-957.

Husain, M., \& Nachev, P. (2007). Space and the parietal cortex. Trends in Cognitive Sciences, 11, 30-36.

Jin, Y., \& Hilgetag, C. C. (2008). Perturbation of visuospatial attention by highfrequency offline rTMS. Experimental Brain Research, 189, 121-128.

Kim, Y. H., Min, S. J., Ko, M. H., Park, J. W., Jang, S. H., \& Lee, P. K. (2005). Facilitating visuospatial attention for the contralateral hemifield by repetitive TMS on the posterior parietal cortex. Neuroscience Letters, 382, 280-285. 
Klingberg, T. (2006). Development of a superior frontal- intraparietal network for visuo-spatial working memory. Neuropsychologia, 44, 2171-2177.

Klingberg, T., Forssberg, H., \& Westerberg, H. (2002). Increased brain activity in frontal and parietal cortex underlies the development of visuospatial working memory capacity during childhood. Journal of Cognitive Neuroscience, 14, 1-10.

Kriegeskorte, N., \& Goebel, R. (2001). An efficient algorithm for topologically correct segmentation of the cortical sheet in anatomical MR volumes. Neuroimage, 14, 329346.

Kveraga, K., Ghuman, A. S., \& Bar, M. (2007). Top-down predictions in the cognitive brain. Brain and Cognition, 65, 145-168.

Lamme, V. A., \& Roelfsema, P. R. (2000). The distinct modes of vision offered by feedforward and recurrent processing. Trends in Neurosciences, 23, 571-579.

Lehmann, C., Vannini, P., Wahlund, L. O., Almkvist, O., \& Dierks, T. (2006). Increased sensitivity in mapping task demand in visuospatial processing using reactiontime- dependent hemodynamic response predictors in rapid event-related fMRI. Neuroimage, 31, 505-512.

Luks, T. L., Sun, F. T., Dale, C. L., Miller, W. L., \& Simpson, G. V. (2008). Transient and sustained brain activity during anticipatory visuospatial attention. NeuroReport, 19, 155-159.

Mayer, A. R., Seidenberg, M., Dorflinger, J. M., \& Rao, S. M. (2004). An event-related fMRI study of exogenous orienting: Supporting evidence for the cortical basis of inhibition of return? Journal of Cognitive Neuroscience, 16, 1262-1271.

Mellet, E., Tzourio, N., Crivello, F., Joliot, M., Denis, M., \& Mazoyer, B. (1996). Functional anatomy of spatial mental imagery generated from verbal instructions. Journal of Neuroscience, 16, 6504-6512.

Muri, R. M., Buhler, R., Heinemann, D., Mosimann, U. P., Felblinger, J., Schlaepfer, T. E., et al. (2002). Hemispheric asymmetry in visuospatial attention assessed with transcranial magnetic stimulation. Experimental Brain Research, 143, 426-430.

Owen, A. M., Evans, A. C., \& Petrides, M. (1996). Evidence for a two-stage model of spatial working memory processing within the lateral frontal cortex: A positron emission tomography study. Cerebral Cortex, 6, 31-38.

Petit, L., Courtney, S. M., Ungerleider, L. G., \& Haxby, J. V. (1998). Sustained activity in the medial wall during working memory delays. Journal of Neuroscience, 18, 94299437.

Petrides, M. (1994). Frontal lobes and behaviour. Current Opinion in Neurobiology, 4, 207-211.

Postle, B. R., Berger, J. S., Taich, A. M., \& DðEsposito, M. (2000). Activity in human frontal cortex associated with spatial working memory and saccadic behavior. Journal of Cognitive Neuroscience, 12(Suppl. 2), 2-14.

Roebroeck, A., Formisano, E., \& Goebel, R. (2005). Mapping directed influence over the brain using Granger causality and fMRI. Neuroimage, 25, 230-242.

Rounis, E., Yarrow, K., \& Rothwell, J. C. (2007). Effects of rTMS conditioning over the fronto-parietal network on motor versus visual attention. Journal of Cognitive Neuroscience, 19, 513-524. 
Rushworth, M. F., Ellison, A., \& Walsh, V. (2001). Complementary localization and lateralization of orienting and motor attention. Nature Neuroscience, 4, 656-661.

Rypma, B., \& DðEsposito, M. (2003). A subsequent-memory effect in dorsolateral prefrontal cortex. Brain Research, Cognitive Brain Research, 16, 162-166.

Sack, A. T., Camprodon, J. A., Pascual-Leone, A., \& Goebel, R. (2005). The dynamics of interhemispheric compensatory processes in mental imagery. Science, 308, 702-704. Sack, A. T., Hubl, D., Prvulovic, D., Formisano, E., Jandl, M., Zanella, F. E., et al. (2002). The experimental combination of rTMS and fMRI reveals the functional relevance of parietal cortex for visuospatial functions. Brain Research, Cognitive Brain Research, 13, 85-93.

Sack, A. T., Jacobs, C., De Martino, F., Staeren, N., Goebel, R., \& Formisano, E. (2008). Dynamic premotor-to-parietal interactions during spatial imagery. Journal of Neuroscience, 28, 8417-8429.

Sack, A. T., Kohler, A., Bestmann, S., Linden, D. E., Dechent, P., Goebel, R., et al. (2007). Imaging the brain activity changes underlying impaired visuospatial judgments: Simultaneous fMRI, TMS, and behavioral studies. Cerebral Cortex, 17, 2841-2852.

Sarter, M., Givens, B., \& Bruno, J. P. (2001). The cognitive neuroscience of sustained attention: Where top-down meets bottom-up. Brain Research, Brain Research Reviews, 35, 146-160.

Scherf, K. S., Sweeney, J. A., \& Luna, B. (2006). Brain basis of developmental change in visuospatial working memory. Journal of Cognitive Neuroscience, 18, 1045-1058.

Talairach, J., \& Tournoux, P. (1988). Co-planar stereotaxic atlas of the human brain: 3-Dimensional proportional system, an approach to cerebral imaging. Stuttgart: Thieme.

Trojano, L., Grossi, D., Linden, D. E., Formisano, E., Goebel, R., Cirillo, S., et al. (2002). Coordinate and categorical judgements in spatial imagery. An fMRI study. Neuropsychologia, 40, 1666-1674.

Trojano, L., Grossi, D., Linden, D. E., Formisano, E., Hacker, H., Zanella, F. E., et al. (2000). Matching two imagined clocks: The functional anatomy of spatial analysis in the absence of visual stimulation. Cerebral Cortex, 10, 473-481.

Valero-Cabre, A., Pascual-Leone, A., \& Rushmore, R. J. (2008). Cumulative sessions of repetitive transcranial magnetic stimulation (rTMS) build up facilitation to subsequent TMS-mediated behavioral disruptions. European Journal of Neuroscience, 27, 765-774.

Yeh, Y. Y., Kuo, B. C., \& Liu, H. L. (2007). The neural correlates of attention orienting in visuospatial working memory for detecting feature and conjunction changes. Brain Research, 1130, 146-157.

Zarahn, E., Aguirre, G. K., \& D’Esposito, M. (1999). Temporal isolation of the neural correlates of spatial mnemonic processing with fMRI. Brain Research, Cognitive Brain Research, 7, 255-268. 


\section{Summary}

Recent debate has focused on whether or not we are our brains. Are we 'just' a bunch of neurons, firing happily away in the illusion of free will and control? Or are we more than this, and is our brain merely the executor of our wishes and commands? It is perhaps a little unfortunate that research focused on 'consciousness' implicitly gets dragged into this largely philosophical debate. After all, studies aiming to link events in our conscious world to changes in our brain are not by definition more or less scientific than studies focused on, for example, word processing or attention. Although one might argue that they are more ambitious in scope as, in the end, consciousness research aims to crack the neural code behind what we most intimately know: our own experiences. There is a reason we place much value on 'what we see with our own eyes'. What do we know best, if not the things we directly experience by our senses? In the current dissertation, this is how we defined 'consciousness': as the percepts of sensory inputs we subjectively experience.

One way to broach such an expansive topic, is to approach the problem from several directions, at several levels, using several complementary tools. Having defined 'consciousness' as subjective experience of sensory inputs, we decided to focus on visual inputs, as the visual system has by now been mapped to an impressive extent. In the Prologue (P.1), we performed a conceptual analysis of the research program focused on finding the 'neural correlates of consciousness', i.e. the brain events that change along with changes in conscious vision. We noted that conscious vision involves mechanisms 'early' in the visual system (Parts I/II), but also 'higher' regions including the frontoparietal network (Part III). In P.1, we suggested that correlational brain research can indeed uncover such 'neural correlates' of consciousness, but that this brings us only part of the way. The uncovered neural events could be neural 'prerequisites' of conscious experience, if they are necessary but not sufficient for a subjective percept, and precede or co-occur in time with the neural 'substrates' of conscious experience. These 'substrates' are in a sense the neural events we would like to identify, since they directly underlie (and/or are identical with) conscious experience. In conventional imaging paradigms, it would be difficult however to separate such neural substrates/prerequisites from neural 'consequences' of consciousness, which follow (from) conscious experience but are neither necessary nor sufficient for the experience itself. Difficult, but perhaps not impossible, which is why we outlined a multi-pronged research program to separate these various 'types' of neural correlates of consciousness.

The experimental work presented in this dissertation was divided in three parts, each approaching the problem of conscious vision in the brain in a different way, on a different level. In Part I, we presented a new visual phenomenon dubbed the 'disrupted rivalry effect' (DRE). Using psychophysics to study mechanisms in the human brain, we adapted the well-established 'binocular rivalry' paradigm to uncover this new finding. In binocular rivalry, a form of bistable vision, the left eye is presented 
with one image, but the right eye is presented with an incompatible image. Rather than fusing both images into one, the brain selects one of the two images for conscious vision. However, it soon 'changes its mind', and the subject perceives the alternative image. This process of perceptual switching between two conscious percepts (the two images presented to the two eyes) continues indefinitely, since no true resolution of the interocular conflict is possible. Using incompatible visual images of stars versus triangles, presented in the periphery (outside of the point of fixation - where the subject gazes), we developed the disrupted rivalry paradigm. One eye is first presented with one image (e.g. triangle) for several seconds. Then the alternative image (e.g. star) is flashed briefly to the other eye, leading to brief conscious perception of this second image. After only a few hundred milliseconds however, this second image is removed, so that again only the initial (triangle) image is presented to (one eye of) the subject. Existing models of binocular rivalry and conscious vision predict that the only remaining visual image should be consciously perceived again. Yet, we found that subjects first see 'nothing' for up to several seconds, until the remaining image finally reappears. In the four chapters of Part I, presenting a sequential set of experiments, we quantified and investigated this visual phenomenon to evaluate its constraints and underlying neuronal mechanisms. While research continues, the interim interpretation is that binocular rivalry mechanisms do underlie this 'disappearance effect', which essentially comes down to continued conscious perception of the (non-)image presented to the eye that received the 'flashed' rivaling image. Yet, it appears that this is not the whole story, as fading and/or filling-in mechanisms may contribute to the surprisingly long duration of the effect. Finally, the phenomenon seems to point to a crucial role of not only visual transient onset signals, in defining conscious visual perception, but also visual transient offset signals, since chapter I.4 demonstrated that simply removing one of the two images during standard binocular rivalry can be sufficient to induce the disrupted rivalry effect.

In Part II, we focused again on mechanisms in early visual cortex, but using a very different approach. Here, we used transcranial magnetic stimulation (TMS) to directly manipulate brain activity in a specific anatomical location (i.e. early visual cortex) at very specific points in time. The 'TMS masking' paradigm traditionally consists of a single visual stimulus presented on a computer screen, followed by a TMS pulse aimed at early visual cortex with the goal of disrupting ongoing neuronal processing. If the timing of the TMS pulse is just right (conventionally around 80-100 milliseconds after the appearance of the visual image), subjects can no longer see the image. Magnetic stimulation of the brain can thus be used to determine what subjects do and do not see. Of course, this is constrained by a specific set of parameters, and requires very brief presentation of already difficult-to-see images in a controlled laboratory setting. Yet, although this TMS masking effect was first reported around 25 years ago, it has been only partially explored since then, and the parameters determining the effect remain unclear. At the same time, the effect could be used to reveal much about the role of early visual cortex in conscious vision. In a sequential series of experiments, described in four chapters of Part II, we therefore explored the TMS masking effect further. We went beyond previous work by not only asking participants to make educated guesses about properties of the visual images and then evaluating 
their accuracy, but also asking them to subjectively rate their conscious perception of the images. While previous literature suggested that these two measures of visual processing (objective 'forced-choice performance' versus subjective 'visibility ratings') might diverge, i.e. might be differentially sensitive to disruption of early visual cortex by TMS, over the whole set of experiments we essentially found a strong correspondence. It thus appears on the basis of these experiments that when TMS disrupts visual processing, it does not selectively disrupt only conscious perception or potentially 'unconscious' guesswork. We also went beyond previous research by evaluating not only the effect of TMS disruption of ongoing neuronal processing after presentation of visual stimuli, but also measuring the effects of TMS pulses applied prior to visual stimuli. We found that TMS pulses applied several tens of milliseconds before a visual image even appears on screen can somehow mask the image from conscious perception as well. Moreover, we showed that, depending on the precise timing of pulses, this masking effect can either be global or retinotopically specific. These results (II.3/II.4), combined with methodological implementations of different types of stimuli (II.2), high effective temporal resolution (II.1), and concurrent work from colleagues in the field (II.5) suggest that the TMS masking effect can yet be used to find out more about how early visual cortex contributes to conscious, and potentially unconscious, visual processing.

While basic mechanisms in early visual cortex are undoubtedly crucial in the determination and development of perception, it is unlikely that these mechanisms by themselves can fully account for conscious vision. Indeed, conventional brain research into the neural basis of conscious perception finds activity in widespread regions of the brain, not only early visual cortex. This widespread activity likely reflects processes directly relevant for seeing, such as the knowledge of object information, processes of attention and/or working memory, or voluntary control over perception. In particular, one functional network that has repeatedly been associated with conscious vision is the frontoparietal network (P.1). In line with the postulated multi-level, multi-tool approach to studying conscious vision in the brain, in Part III we probed this frontoparietal network and its associated functions using a variety of methods. Specifically, we tested its functional relevance (III.1), connectivity (III.4), and associated role of spatiotemporal attention (III.3), in the context of conscious vision and its cognitive backdrop.

Focusing on the previously suggested role of frontoparietal regions in bistable vision (e.g. as in binocular rivalry, but in this case using a bistable rotating sphere that can be perceived as rotating clockwise or counter-clockwise), we applied TMS to manipulate the excitability (and by extension activity) of these regions in the right hemisphere in chapter III.1. We could not affirm previous suggestions that frontal (or parietal) cortex drives automatic conscious percept switches during passive bistable vision. Yet, while many participants normally have a measure of voluntary control over bistable perception (making the percept switch at will), this voluntary switching behavior was reduced after TMS over frontal cortex. Thus, frontal cortex appears functionally relevant for voluntary switching of conscious percept, but the very same region did not seem crucial for automatic percept switching. The juxtaposition of these findings was discussed more deeply in a conceptual setting in III.2. Voluntary control 
over conscious perception does show that cognitive mechanisms can affect conscious perception. As mentioned, the frontoparietal network consistently appears to play a role in such influences on visual processing. Given the widespread nature of this network, this role likely involves communication between brain regions. Such communication in the brain is also called 'connectivity' (III.4), and likely engages oscillatory processes (III.3). In III.4 we used functional magnetic resonance imaging (fMRI) to study connectivity within the frontoparietal network in higher-order vision. More specifically, we used Granger Causality Mapping to evaluate information flows within the frontoparietal network underlying visuospatial judgments. Frontal brain regions sent information to parietal regions, during a visuospatial judgment task, but not a color judgment task. Thus showing that frontoparietal connectivity depends on the visual task at hand, future work could use this methodology to zoom in on conscious vision more specifically.

Using fMRI to study fast neuronal mechanisms of communication is difficult, due to limitations in temporal resolution. Magnetoencephalography (MEG) on the other hand can track brain activity with temporal specificity in the order of milliseconds. MEG can therefore be used to track 'brain waves', oscillations in activity in particular frequency bands. The frontoparietal network is not only implicated in conscious vision but also in mechanisms of spatiotemporal attention (P.1); attention and consciousness are thus difficult to separate (P.1). Given the role of oscillations in neuronal communication and selection, our last approach towards the establishment of conscious vision in the brain was to study brain oscillations in the alpha-frequency-band $( \pm 8$ $12 \mathrm{~Hz}$ ), a frequency previously associated with both attention and conscious vision, and with parietal cortex. In chapter III.3 we attempted to manipulate neuronal alpha oscillations psychophysically, to bias conscious perception. By presenting rhythmic visual images at alpha frequency (versus several control 'flanker' frequencies) we hypothesized to 'align', or 'phase-lock' alpha oscillations in the brain. Following this, we presented visual targets at various latencies. In these experiments we noticed that 1) visual attention and associated task performance were specifically sensitive to preceding trains of visual cues in alpha frequency, and 2) visual task performance itself 'oscillated' at alpha frequency after an alpha-train of visual cues. Moreover, using MEG on a different day to identify participants' individual specific alpha frequencies, we could link the observed alpha waves in visual task performance with the alpha waves in the brains of the same subjects.

In sum, we could show that indeed a range of processes in the human brain, such as cognitive mechanisms in frontoparietal cortex, oscillations at particular frequencies, as well as lower-level time-specific processes in early visual cortex and specific competitive mechanisms between early eye-pathways, all contribute to the conscious visual percept we experience at any point in time. It appears that further indepth research on each of these levels of the visual system is required to identify and further outline these various contributions. Yet, a bird's-eye view is also necessary, to link and integrate these various fields of research using the arsenal of brain research tools applied here, if we intend to eventually build a comprehensive theory of conscious vision in the human brain. Lastly, a valid future theory will undoubtedly require intense collaboration of empirical researchers on the one hand, with analytical experts in 
philosophy and epistemology on the other hand. Personally I hope that, in time, the interdisciplinary efforts of the international community will lead to that theory. In time, we may truly understand how our brains give rise to our world. After all, even if we are not our brains, we sure aren't much without them. 
Brain in Sight 


\section{Samenvatting}

Er is recent veel discussie over of wij nu wel of niet 'ons brein zijn'. Zijn we 'slechts' een constellatie van vrolijk vurende neuronen, gevangen in een illusie van vrije wil en controle? Of zijn we Meer dan dat en is ons brein enkel de uitvoerder van onze wensen en opdrachten? Het is misschien een beetje onfortuinlijk dat onderzoek naar 'bewustzijn' soms impliciet wordt meegezogen in dit leuke maar grotendeels filosofische debat. Tenslotte zijn experimenten die gebeurtenissen in onze belevingswereld willen relateren aan veranderingen in ons brein niet per definitie meer of minder wetenschappelijk dan onderzoek naar, bijvoorbeeld, aandacht of woordverwerking. Men zou misschien wèl kunnen stellen dat bewustzijnsonderzoek op een bepaalde manier ambitieuzer of grootschaalser is, aangezien het uiteindelijke doel niets minder is dan het kraken van de neuronale code achter wat wij het meest intiem kennen: onze subjectieve ervaringen. Niet voor niets vertrouwen we het meest op dingen 'die we met eigen ogen zien'. Wat kennen we beter dan onze directe subjectieve beleving? In dit proefschrift omschrijven we 'bewustzijn' als de percepten van sensorische inputs die we subjectief ervaren.

Eén manier om een dergelijk omvangrijk onderwerp te benaderen is door verschillende invalshoeken te nemen, op verschillende niveaus, met behulp van complementaire instrumenten. $\mathrm{Na}$ 'bewustzijn' te hebben gedefinieerd als subjectieve ervaring van sensorische inputs besloten we om ons te beperken tot visuele inputs, aangezien het visuele systeem in het brein inmiddels tot in indrukwekkend detail in kaart is gebracht. In de Proloog voerden we een conceptuele analyse uit van het onderzoeksprogramma naar de 'neurale correlaten van bewustzijn', ofwel de gebeurtenissen in de hersenen die samengaan met gebeurtenissen in bewuste waarneming. We merkten op dat bewuste visie geassocieerd is met processen in 'vroege' visuele gebieden (Deel I/II) maar ook in 'hogere' gebieden van het visuele systeem, waaronder het frontoparietale netwerk (Deel III). In P.1 suggereerden we dat correlationeel hersenonderzoek dan wel zulke 'neurale correlaten' van bewuste waarneming kan uitwijzen, maar dat dit slechts deel van de verklaring zal zijn. De op die manier gevonden hersenprocessen kunnen neurale 'vereisten' zijn van bewuste waarneming, als ze wel nodig maar niet afdoende zijn voor een subjectieve ervaring en tegelijk met, of voorafgaand aan, neurale 'substraten' van de bewuste ervaring plaatsvinden. Deze 'substraten' zijn tot op zekere hoogte de processen die we werkelijk willen identificeren, aangezien zij direct ten grondslag liggen aan (of identiek zijn aan) bewuste ervaring. In conventionele imaging paradigma's is het moeilijk om dergelijke substraten/vereisten te scheiden van neurale 'gevolgen' van bewuste waarneming, welke plaats hebben na (of deels tegelijk met) een bewuste ervaring, maar zelf niet nodig en ook niet voldoende zijn voor de ervaring. Moeilijk, maar wellicht niet onmogelijk: daarom schetsten we een meerdelig onderzoeksprogramma voor de nabije toekomst dat in staat kan zijn om neurale 'vereisten', 'substraten' en 'gevolgen' van bewuste ervaring te scheiden. 
Het experimentele werk van dit proefschrift is opgedeeld in drie stukken, elk met een andere benadering tot het probleem van bewuste visie in het brein. In Deel I presenteerden we een nieuw visueel fenomeen dat we het 'onderbroken rivalry effect' hebben genoemd. Gebruik makend van psychofysische methoden om het visuele systeem in het brein te onderzoeken, pasten we het gevestigde 'binocular rivalry' paradigma aan. In binocular rivalry, een form van bistabiele visie, wordt een plaatje gepresenteerd aan het linker oog dat niet hetzelfde en niet compatibel is met een tweede plaatje dat wordt gepresenteerd aan het rechter oog. In plaats van dat een fusie wordt waargenomen van beide plaatjes lost het brein dit op door slechts 1 van de twee plaatjes te selecteren voor bewuste waarneming. Dit duurt echter niet lang, want al snel verandert het brein van gedachten en ziet de proefpersoon het alternatieve plaatje. Dit proces van wisselende perceptie van het ene, danwel andere plaatje, duurt oneindig voort aangezien er geen voor het brein bevredigende resolutie is van het interoculaire conflict.

We maakten hier gebruik van plaatjes van sterretjes tegenover driehoekjes, die we presenteerden in de periferie (het visuele veld buiten het punt van fixatie - waar de proefpersoon heen kijkt - dus lichtelijk in de 'ooghoek' van de waarnemer). In het onderbroken rivalry paradigm wordt eerst 1 plaatje (bijv. Driehoek) gepresenteerd aan 1 oog, voor enkele seconden. Dan wordt het 2e plaatje (dus Ster) gepresenteerd aan het 2e oog, waardoor het als 'nieuwe' informatie meteen waargenomen wordt. Maar na slechts een paar honderd milliseconden wordt het tweede plaatje weer weggehaald, zodat wederom enkel het eerste plaatje gepresenteerd wordt. Huidige modellen van binocular rivalry stellen dat op dit moment het eerste plaatje weer wordt waargenomen, aangezien het de enige werkelijk input van het visuele systeem vormt. Echter, wij vonden dat proefpersonen eerst 'niets' zien voor soms seconden lang. In the vier hoofdstukken van Deel I, waarin een serie experimenten wordt beschreven, kwantificeerden en onderzochten we dit fenomeen om de onderliggende mechanismen en neuronale processen te evalueren. Het onderzoek gaat voort, maar de interim conclusie is dat eerder gepostuleerde binocular rivalry processen een rol spelen bij dit effect, maar dat ze niet de lange duur van het 'verdwijn-effect' kunnen verklaren. Andere mechanismen, zoals 'fading' en/of 'filling-in' kunnen wellicht uitkomst bieden, maar dat moet nog verder onderzocht worden. In ieder geval lijkt het er op dat nog altijd de informatie van het tweede 'geflitste' oog wordt geselecteerd voor bewuste visie. Het fenomeen wijst ook op een mogelijk cruciale rol voor 'offset-signalen' in het bepalen van wat geselecteerd wordt voor bewuste waarneming, naast 'onset-signalen', aangezien we in hoofdstuk I.4 aantoonden dat enkel het weghalen van een stimulus tijdens conventionele binocular rivalry al voldoende kan zijn voor het verdwijn-effect.

In Deel II richtten we ons wederom op mechanismen in vroege visuele gebieden, maar gebruikten we een heel andere onderzoeksmethode. Hier gebruikten we transcraniale magnetische stimulatie (TMS) om hersenactiviteit op een specifieke anatomische locatie (namelijk vroege visuele cortex) op specifieke punten in tijd te beïnvloeden. Het 'TMS masking' paradigma begint traditioneel met een visuele stimulus (plaatje) die kort op een scherm wordt gepresenteerd. Deze wordt gevolgd door een TMS puls gericht op visuele cortex opdat de neuronale activiteit precies op dat moment verstoord wordt. Als de timing van deze verstoring precies juist is (traditioneel 
rond 80-100 milliseconden na verschijnen van het visuele plaatje) zijn proefpersonen niet in staat het plaatje waar te nemen. Op deze manier kan TMS dus gebruikt worden om te bepalen wat proefpersonen wel en niet bewust zien. Natuurlijk is dit wel sterk beperkt door een specifieke set parameters en is het noodzakelijk dat het plaatje, dat hoe dan ook klein en moeilijk zichtbaar is, slechts zeer kort gepresenteerd wordt in een gecontroleerde labomgeving. Hoewel dit TMS masking effect al ongeveer 25 jaar geleden voor het eerst werd gerapporteerd is het nog altijd niet volledig onderzocht en blijven de parameters die van belang zijn voor specifieke resultaten onduidelijk. En dat terwijl het effect mogelijk veel bij kan dragen aan het begrijpen van de rol van vroege visuele cortex in het ontstaan van bewuste visie. Daarom ondernamen we een serie experimenten, beschreven in vier hoofdstukken van Deel II, waarin we dit maskeer effect verder onderzochten. We gingen verder dan voorgaand onderzoek door proefpersonen niet alleen te vragen bepaalde aspecten van het plaatje te beoordelen, maar ze ook direct te vragen om een subjectief oordeel te vellen over hun bewuste waarneming van de visuele stimulus. Hoewel eerdere rapporten suggereerden dat deze twee maten van visuele informatieverwerking (objectieve 'forced-choice performance' en subjectieve 'zichtbaarheids-ratings') uiteen kunnen lopen, ofwel eventueel niet even ontvankelijk zouden kunnen zijn voor de verstoring door de magnetische puls, vonden wij over het algemeen een sterke correspondentie tussen de twee gedragsmaten. Op basis van onze experimenten lijkt het er dus niet op dat occipitale TMS verschillende effecten heeft op bewuste visuele perceptie tegenover mogelijk 'onbewust gokgedrag'. Een tweede manier waarop we verder gingen dan veel voorgaand TMS masking onderzoek was door TMS pulsen niet alleen toe te dienen op de eerdere bekende momenten na presentatie van de visuele stimulus, maar in andere condities ook op momenten voordat het plaatje überhaupt in beeld was. We vonden hierbij dat TMS pulsen, indien tientallen milliseconden voor presentatie van het plaatje toegediend, op een of andere manier ook kunnen zorgen voor maskering van de visuele stimulus. Dit is verwonderlijk aangezien de visuele informatie pas een behoorlijke tijd later zal 'arriveren' in het met TMS verstoorde gebied. Afhankelijk van de precieze timing van deze TMS pulsen bleek dit TMS masking effect danwel globaal, danwel retinotopisch specifiek te zijn. Deze resultaten (II.3/II.4), in combinatie met methodologische implementaties van verschillende soorten stimuli (II.2), hoge effectieve temporele resolutie (II.1) en in samenhang met simultaan werk van collega's (II.5), suggereren dat het TMS masking effect nog altijd gebruikt kan worden om meer te ontdekken over hoe vroege visuele hersengebieden bijdragen aan bewuste, en mogelijk onbewuste, visuele verwerking.

Hoewel basale mechanismen in vroege visuele cortex cruciaal blijken te zijn voor het bepalen en de ontwikkeling van bewuste waarneming is het onwaarschijnlijk dat deze mechanismen de volledige verklaring van bewuste visie kunnen vormen. Zo vindt hersenonderzoek naar de neurale basis van bewuste waarneming meestal activiteit verspreid over veel gebieden in het brein, niet alleen in vroege visuele cortex. Deze activiteit is waarschijnlijk betrokken bij processen die direct van belang zijn voor visuele waarneming, zoals kennis over objecten en categorieën, allocatie van aandacht, of bijvoorbeeld moedwillige controle over perceptie. Eén functioneel netwerk in het bijzonder is vaak geassocieerd met bewuste waarneming; het frontoparietale netwerk 
(P.1). In lijn met de voorgestelde multi-level, multi-methodologische benadering van bewuste visie in het brein hebben we dit frontoparietale netwerk met geassocieerde functies onderzocht op verscheidene niveaus met verschillende instrumenten, zoals gerapporteerd in Deel III. Specifieker; we onderzochten de functionele relevantie (III.1), connectiviteit (III.4), en de geassocieerde rol van spatiotemporele aandacht (III.3), in de context van bewuste visuele waarneming en haar cognitieve achtergrond.

Met een focus op de eerder voorgestelde rol van frontoparietale gebieden in bistabiele visie (waarvan binocular rivalry een voorbeeld is, hoewel we in dit geval een bistabiele roterende bol gebruikten die waargenomen kan worden als ofwel roterend met de klok mee ofwel tegen de klok in), dienden we TMS toe om de activiteit van deze gebieden in de rechter hemisfeer te beïnvloeden, in hoofdstuk III.1. We konden niet bevestigen dat frontale (of parietale) cortex automatische wisselingen in percept induceert, al was dit wel voorgesteld in de literatuur. Echter, hoewel veel proefpersonen normaal gesproken een bepaalde moedwillige controle kunnen uitoefenen op hun bistabiele waarneming (ze kunnen de bol vaker laten 'omdraaien' in rotatie-richting, als ze dat willen), was deze cognitieve vaardigheid verminderd na TMS over frontale cortex. Het lijkt er dus op dat frontale cortex functioneel relevant is voor moedwillige invloed op bewuste waarneming, terwijl precies datzelfde gebied niet cruciaal betrokken lijkt te zijn bij automatische wisselingen van percept. De tegenstelling van deze bevindingen werd dieper besproken in een conceptuele setting in hoofdstuk III.2. Puur het feit dat proefpersonen in staat zijn bewust hun waarneming te beïnvloeden laat al zien dat cognitieve mechanismen invloed hebben op bewuste visuele waarneming. Zoals gezegd is een hypothese dat het frontoparietale netwerk consistent een rol speelt bij dit soort invloeden op visuele waarneming. Gezien het verspreide karakter van dit netwerk, met gebieden die anatomisch aanzienlijk uit elkaar liggen, lijkt communicatie tussen gebieden binnen dit netwerk een cruciale rol te spelen. Zulke communicatie wordt ook wel omschreven als 'connectiviteit' (III.4), en heeft waarschijnlijk te maken met oscillaties (III.3) als communicatiemechanisme. In III.4 gebruikten we functionele magnetische resonantie imaging (fMRI) om connectiviteit binnen het frontoparietale netwerk te bekijken tijdens cognitieve visuele taken. Specifieker: we gebruikten Granger Causality Mapping om informatiestromen binnen het frontoparietale netwerk te bekijken die ten grondslag liggen aan visuospatiele oordelen. Frontale hersengebieden zonden informatie naar parietale gebieden, specifiek tijdens visuospatiele oordelen en niet tijdens het beoordelen van kleur. Dit laat zien dat frontoparietale connectiviteit afhankelijk is van de visuele taak die uitgevoerd wordt, wat de basis legt voor toekomstig onderzoek middels deze methode naar interacties tijdens specifiek bewuste visuele processen.

Het gebruik van fMRI om snelle neuronale mechanismen van communicatie te bestuderen is moeilijk wegens de beperkte temporele resolutie. Magnetoencephalografie (MEG), aan de andere kant, kan hersenactiviteit bijhouden met een temporele specificiteit van milliseconden. Hierdoor kan MEG gebruikt worden om 'hersensgolven' te meten: oscillaties van activiteit in bepaalde frequenties. Het frontoparietale netwerk is niet alleen geassocieerd met bewuste visie maar ook met mechanismen van spatiotemporele aandacht (P.1), wat het moeilijk maakt om aandacht en bewustzijn in het brein te onderscheiden. Gezien de rol van oscillaties in neuronale 
communicatie en selectie was onze laatste benadering van bewuste waarneming en onderliggende factoren het bestuderen van oscillaties in een bepaalde frequentie. Deze alfa-frequentie $( \pm 8-12 \mathrm{~Hz})$ is herhaaldelijk geassocieerd met zowel aandacht als bewuste visuele waarneming en lijkt dicht verbonden met parietale cortex. In hoofdstuk III.3 manipuleerden we neuronale alfa oscillaties psychofysisch, om bewuste waarneming te beïnvloeden. Door visuele plaatjes ritmisch aan te bieden met alfa-frequentie (tegenover 'buur-frequenties' als controle) hoopten we alfa oscillaties in het brein te vangen in dat ritme, wat we 'phase-locking' van de oscillaties noemen. $\mathrm{Na}$ de ritmische plaatjes presenteerden we visuele targets op verschillende momenten, waarop proefpersonen een taak moesten uitvoeren. In deze experimenten zagen we dat 1) visuele aandacht en geassocieerde taakuitvoering specifiek gevoelig waren voor voorgaande plaatjes gepresenteerd in de alfa-frequentie en 2) het succes van de visuele taak oscilleerde zelf met alfa-frequentie na de alfa-trein van voorgaande plaatjes. Bovendien gebruikten we MEG op een andere dag om de individuele alfa golven in het brein van dezelfde proefpersonen te meten, en konden we vervolgens laten zien dat de individuele oscillaties in het visuele gedrag gerelateerd waren aan de oscillaties in het brein.

Samenvattend konden we beschrijven in dit proefschrift hoe een hele rits aan processen in het menselijk brein, waaronder cognitieve processen in het frontoparietale netwerk, oscillaties met bepaalde specifieke frequenties, evenals tijdspecifieke mechanismen in vroege visuele cortex en vroege competitieve mechanismen tussen de informatiestromen van de twee ogen, allemaal hun bijdrage leveren aan het uiteindelijke bewuste visuele percept dat we waarnemen. Het is duidelijk dat verder fundamenteel onderzoek op elk van deze niveaus van het visuele system nodig is, gebruik makend van het volledige arsenaal van hier toegepaste onderzoeksmethoden, als we uiteindelijk een volledige theorie willen ontwikkelen van bewuste waarneming in het menselijk brein. Daarnaast lijkt het onvermijdelijk dat het ontstaan van een dergelijk theorie afhankelijk zal zijn van een intense samenwerking tussen empirische onderzoekers aan de ene kant met analytische experts in filosofie en epistemologie aan de andere kant. Persoonlijk hoop ik dat dergelijke interdisciplinaire investeringen in de toekomst deze theorie mogelijk zullen maken. Ooit zullen we wellicht werkelijk begrijpen hoe ons brein leidt tot onze intieme belevingswereld. Ten slotte, of we nu onze hersenen zijn of niet, we zijn zeker niet veel zonder ze. 


\section{Acknowledgments}

What a ride...

I've heard post-doctoral researchers claim that you don't realize as a $\mathrm{PhD}$ student how much time you really have, how easy it actually is, in hindsight. Honestly? I don't believe them. I'm sure things will continue to be difficult from here on, but I wonder if these guys have not simply forgotten what it was like to do a PhD. Yet somehow, here it is. I've had to learn a whole lot, about a very wide range of things, to get from my first day to this one. I could not have done it alone, so I am glad to include in this report of my learning curve a hats-off to the many people that made it possible.

It's difficult to start these acknowledgements because there are so many people I owe substantial and significant debts to. It's impossible to decide which are the steepest, so I will go in something of a chronological order. That leads me firstly to my parents. Thank you mom, for instilling in me an appreciation for science and knowledge. From your relentless badgering about correct citations in high school projects, to an excitement about figuring things out, to meta-discussions on what constitutes real science, all of your help and influences have been invaluable. Yet, all are overshadowed by the immovable emotional anchor and bottomless well of positivism and joie de vivre that you have been. You know how much I needed you for this, and probably always will. Thank you. Long live abundance! Thank you dad, for the complementary lessons you gave me. Your perfect examples of work ethic, passion for the job, and overal sense of duty, have in the end begun to rub off on me. And of course, my interest in the brain comes from you. You know how I feel about father-son stuff. I think that my studying the human brain is just me picking the job that is closest to what you do, without having to study medicine. To both my parents; nothing ever beats the pride on your faces, but thanks for being there for me no matter what. My brothers, Bob, Paul, have been a joy and inspiration to a much larger extent than they know. I love many things in life, but nothing is more awesome than Broertjesavond. Even if all we ever do for the rest of our lives involves spare ribs and games that will be enough for me. Yet, more and more the both of you have become true friends, and the increasing importance of your advice and support has surprised me. Thank you for reminding me of what matters. The same goes for my other family, from grandparents to aunts and uncles (I want to especially mention Kitty), to cousins and recently a beautiful niece: thank you for being important.

From family to friends, which is just more family, really. Job and Michelle, you are amazing. We have done so much together, over the past few years, that they ended up putting us in the same office. I sometimes wonder what people must think... I know it probably seems a little silly to you, but the title of my thesis was Brain in Sight for you - the translated original name of our ambitious project. I am as proud of what we achieved together, and what we dared to strive for, as I am of this dissertation. And our project equally reflects my period as a $\mathrm{PhD}$ student as an indispensable part of my development. Blahblah, I just love you guys. Job, you are so much like a brother to me 
that I have begun to treat you like one, which is not always a good thing. Just know that the more I piss you off, the more it means I like you. Michelle, you know how important you are to me. We've known each other for a very long time, and how careful I have always been to not hurt your feelings (kind of the opposite to Job) - and particularly the fact that I've generally succeeded in this - should say enough. It seems that we will soon part ways, but not really.

If Job and Michelle were my office-siblings, Alex was my office-dad. That kind of sounds weird, but then again they didn't coin the term 'doktervater' for nothing. Alex, you have been my mentor since before my $\mathrm{PhD}$ started, and were instrumental in getting me this opportunity. You know that I have changed much since we first met (less hair), but you were always there for me. I have always appreciated this, and will never take it for granted. To have had a supervisor with a door that was always open, a chair that was always inviting, and a willingness to make time for everything from the most ambitious of ideas to the silliest of worries, has been a very rare and unique privilege. Besides, which other supervisor always replies to emails within a day? Thank you for your continued support, I am grateful. My second supervisor was Rainer. Rainer, you may or may not know how important you have been for me throughout my $\mathrm{PhD}$. You have been there as an example, and you have been there as an inspiration. Just talking to you, about ideas, about science, about Apple, always left me more than a little excited. This is no small thing; excitement is indispensible for a $\mathrm{PhD}$ dissertation to see the light of day. Your excitement and dreams made everything seem possible, and continue to do so. I love working together with you on stuff, most recently the App, and felt very honored that you always managed to find time in your schedule for me. In terms of supervisors, I also want to mention Gregor Thut. When I visited Glasgow for six months, I expected much in terms of research expertise on the basis of our few prior meetings. What I did not expect was to be received so warmly and openly. Thank you so much for inviting me to dinner, for taking me under your wing, and for introducing me to your family. But also, please know how much I learned from you. I did not expect that my short stay abroad would leave such a mark on me; in Glasgow, and since then in our continued collaboration, you actually had a huge impact on my scientific thinking, my scientific writing, and my overall perspective. It has been extremely valuable. I also want to thank Raymond van Ee, for valuable feedback and discussions and getting me excited about binocular rivalry in recent years. Lastly, Rob, thank you for your help over the years in making decisions, clarifying my thinking, and getting enough chocolate. You have been a very large influence in many ways; without you I possibly would not have been in Maastricht and I definitely would not have gotten excited about consciousness. Who knows what would have happened to me?!

This book is too thick, so I need to speed up. I want to thank my real friend Martin. Buddy, you don't like to pat yourself on the back, but you kept me sane. I don't know if it's the $\mathrm{Oz}$, or just you, but you made me feel that things were generally going to be ok, were generally not so very important actually, and life was generally pretty damn good overall. Honestly, I've gotten considerably more stressed since they put me in a different office, and I don't think that is because of my new roommates as much as it is because you're not in it. Over the years, we've become really good friends, whether you like it or not. Aude, that goes for you too. Always friendly and always with the best 
intentions towards everyone and anyone. You are not only a really wonderful person, you are also an inspiration in this way. And you too, made this thesis possible. Martin and Aude, I hope we remain friends. Nick is now gone, yet he had a role to play as well. Together with Martin, he kickstarted my PhD outside the office, with FIFA nights (sorry for kicking your virtual butt) and dinners. Thanks for the many invitations to Aachen, and for the few times I actually came. Let's just call it even, considering all the times you beat me at poker. And of course, we'll always have Flagstaff... Teresa is not only my office-mom, but actually the mom of the whole TMS group. And now actually Mom with capital M. You, more than anyone, made it actually FUN to go to the office, livening up our workday with your visits and taking my mind of work at the crucial moments (that can be read in two ways). We too have actually become friends, and I am very glad for it. I've also become closer and closer with other colleagues in the TMS group, and many of them have made this thesis possible and more or less direct ways. Christianne, first and foremost, actually contributed directly. But also indirectly, you have no idea how much you shaped me during my PhD. Much of what I have learned, I have actually learned from you! Thank you so much, I hope we continue to work together, as we make a really good team. Another good team was formed more recently, with Felix. Felix, I really enjoy working together and I feel that we can actually achieve really awesome stuff in the near future. To Nina, Katie, Dennis and Eline, Daan, Franziska, Rosanne, Sanne, Jeannette, and to a bunch of students I worked with over the years, thanks for your help, in measuring, thinking, discussing, and all other sorts of doing science with me.

To all my other colleagues, Anke, Federico, Alard, Vincent, Joel, Giancarlo, Francesco, Kamil (stop winning on poker night), and everyone not mentioned by name (for which sorry, it would become a very long list), my sincerest gratitude for making the $\mathrm{CN}$ department a very special place, and an amazing place to learn. I might have gone for a PhD somewhere else, but I am 100\% sure I would not have enjoyed it this much.

Simone, you know me for what I am. And you know that if I let myself go, this paragraph would get so excessively sentimental it would gross everybody out. I know you don't want me to do that, so I shall proceed with reluctant yet rigorous restraint. But it is important that you know that of all the people that made this thesis possible, you were the one person that was irreplaceable. And not only for the thesis. You have made me a better person, and continue to do so every day. Thank you for being you. And for helping me be me. You know the rest. 


\section{Publications}

de Graaf, T. A., Gross, J., Paterson, G., Rusch, T., Sack, A.T., Thut, G. (2013). Alphaband rhythms in visual task performance: phase-locking by rhythmic sensory stimulation. PloS ONE (accepted)

de Graaf, T. A., Hsieh, P. J., \& Sack, A. T. (2012). The 'correlates' in neural correlates of consciousness. Neurosci Biobehav Rev, 36(1), 191-197.

de Graaf, T. A., Goebel, R., \& Sack, A. T. (2012). Feedforward and quick recurrent processes in early visual cortex revealed by TMS? Neuroimage. 61(3):651-9

de Graaf, T. A., \& Sack, A. T. (2011). Null results in TMS: from absence of evidence to evidence of absence. Neurosci Biobehav Rev, 35(3), 871-877.

de Graaf, T. A., de Jong, M. C., Goebel, R., van Ee, R., \& Sack, A. T. (2011). On the functional relevance of frontal cortex for passive and voluntarily controlled bistable vision. Cereb Cortex, 21(10), 2322-2331.

de Graaf, T. A., Herring, J., \& Sack, A. T. (2011). A chronometric exploration of highresolution 'sensitive TMS masking' effects on subjective and objective measures of vision. Exp Brain Res, 209(1), 19-27.

de Graaf, T. A., Cornelsen, S., Jacobs, C., \& Sack, A. T. (2011). TMS effects on subjective and objective measures of vision: stimulation intensity and pre- versus poststimulus masking. Conscious Cogn, 20(4), 1244-1255.

de Graaf, T. A., Roebroeck, A., Goebel, R., \& Sack, A. T. (2010). Brain network dynamics underlying visuospatial judgment: an FMRI connectivity study. $J$ Cogn Neurosci, 22(9), 2012-2026.

de Graaf, T. A., Jacobs, C., Roebroeck, A., \& Sack, A. T. (2009). FMRI effective connectivity and TMS chronometry: complementary accounts of causality in the visuospatial judgment network. PLoS One, 4(12), e8307.

Jacobs, C., de Graaf, TA, Goebel, R., Sack, A.T. (2012). The temporal dynamics of early visual cortex involvement in behavioral priming. PLOS ONE. 7:11. E48808 Machado, S., Araujo, F., Paes, F., Velasques, B., Cunha, M., Budde, H., ..., de Graaf, TA et al. (2011). EEG-based brain-computer interfaces: an overview of basic concepts and clinical applications in neurorehabilitation. Rev Neurosci, 21(6), 451-468.

Peters, J., Reithler, J., Schuhmann, T., de Graaf, TA., Uludag, K., Goebel, R., Sack, AT. (accepted). On the feasibility of concurrent human TMS-EEG-fMRI measurements. Journal of Neurophysiology. 


\section{Curriculum Vitae}

Tom de Graaf was born in Haarlem, the Netherlands, on September $25^{\text {th }} 1983$. After finishing high school at the st. Oelbert gymnasium in Oosterhout, the Netherlands, he lived in Rochester, New York, United States of America, for a year on exchange. In 2003 he started the bachelor program at the Faculty of Psychology and Neuroscience (FPN), Maastricht University. Finishing Cum Laude in 2006, he started the research master program in Cognitive Neuroscience, Neuropsychology, and Psychopathology at the same faculty. Finishing Cum Laude in 2008, he was fortunate enough to be awarded a Toptalent grant by NWO to study conscious vision in the human brain for a $\mathrm{PhD}$ project. This research was performed at the cognitive neuroscience department of FPN, under supervision of Prof Dr. Alexander Sack and Prof Dr. Rainer Goebel, and for six months at Glasgow University under supervision of Prof Dr. Gregor Thut. This research is presented in this dissertation, and is now continued at the same institution by Tom as a postdoctoral fellow. He also continues his work on the BrainMatters project (www.brainmatters.nl), which aims to share neuroscience with as many people as possible. 